Walk This Way: A Kinematic Point-Light Investigation of Victim Vulnerability by

\title{
Brittany Blaskovits
}

A thesis submitted to the Faculty of Graduate and Postdoctoral

Affairs in partial fulfillment of the requirements

for the degree of

Master of Arts

in

Psychology

Carleton University
Ottawa, Ontario

(C) 2015

Brittany Blaskovits 


\begin{abstract}
Research suggests that certain individuals exhibit vulnerability through their gait, and that observers select such individuals as those most vulnerable to victimization. It is currently assumed that the vulnerable gait pattern is an expression of one's submissiveness. Study 1 utilized kinematic point-light display to record individuals walking. The findings suggested that victimization history was related to gait vulnerability. The results also indicated that, contrary to expectation, individuals with more vulnerable features in their gait were more likely to self-report dominant personality characteristics, rather than submissive characteristics. In Study 2, a sample of observers watched the point-light recordings and rated the walkers on their vulnerability to victimization. The results suggested that observers agreed on which walkers were easy targets; they were also accurate in that the walkers that they rated as most likely to experience victimization tended to exhibit vulnerable gait cues. Implications of the findings are discussed.
\end{abstract} Keywords: victim selection, vulnerability, personality, affect, point-light display 


\section{Acknowledgements}

Foremost, I would like to express my gratitude to my supervisor Dr. Craig Bennell. I think it is rare for an advisor to allow a student the freedom to pursue their own vision. I could never have imagined how much I would grow to love research. Thank you for believing in this project and supporting me as I learned. You opened up a world of opportunities, and generated in me, a drive to succeed. Without your patience and hard work, I know this thesis would not have been possible.

I would also like to thank my committee member Dr. Adelle Forth for her guidance and commitment to the project; Dr. Forth's thoughtful consideration was paramount in making this thesis the best it could be. I am also obliged to my external examiner Dr. Karen Schwartz and my thesis chair Dr. Michael Wohl for taking the time to immerse themselves in this research.

Thank you to the administrative staff, in particular Etelle Bourassa and Jessica Whitney. Your hard work does not go unnoticed.

I must extend my sincere appreciation to my friends and lab mates who have kept me smiling over the last two years: Kristen White, Janelle Lebreton, Danielle Labhardt, Rebecca Mugford, Holly Ellingwood, and Natasha Korva. Thank you for being there to share classes, wine, conference rooms, edits, projects, and cue cards. You quickly became my "away" family, and I am lucky to have met and worked with all of you.

Finally, I am deeply indebted to the Carleton School of Information Technology. I am extremely grateful to Dr. Anthony Whitehead who provided the necessary technological resources and methodological expertise. Thank you to my technician 
Andrew Richardson, who fought for camera quality, volunteers, and proper data. Lastly, thank you to Christopher Clarke for the large coffees, and room bookings. 


\section{Dedication}

I would like to dedicate my Master of Arts thesis to my parents Donna and Steve, and my younger brother Cole. I would never have accomplished what I have without your unrelenting belief in me. Thank you for your encouragement, patience, and support. You inspire me every single day. 
Table of Contents

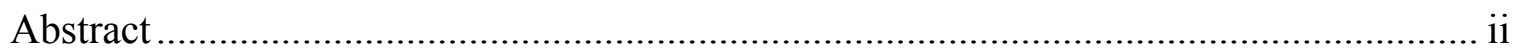

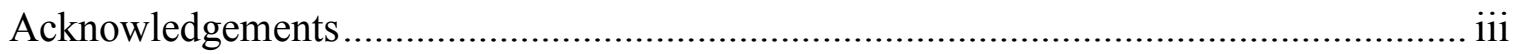

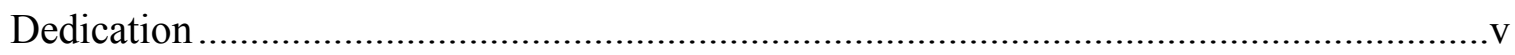

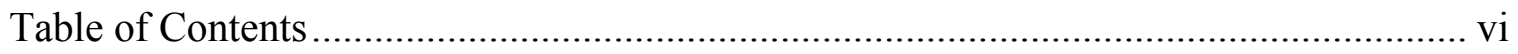

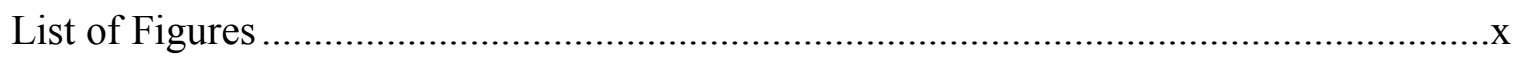

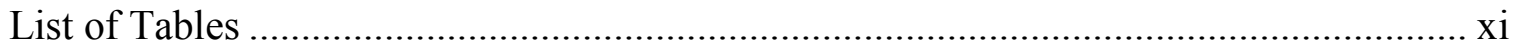

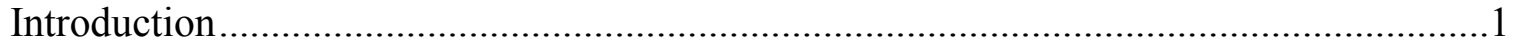

Nonverbal Communication......................................................................

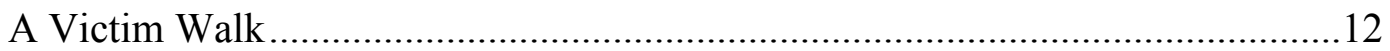

Easy Prey: An Evolutionary Perspective ........................................................16

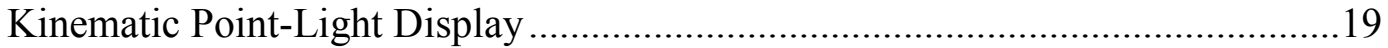

Personality and Victim Selection................................................................24

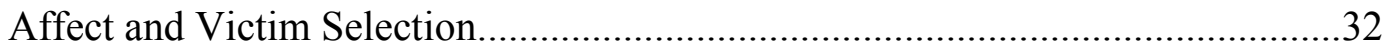

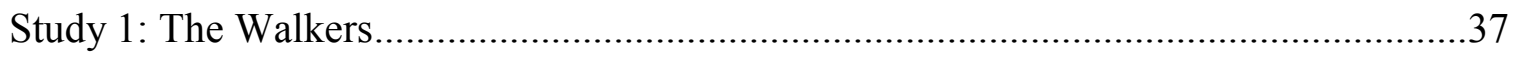

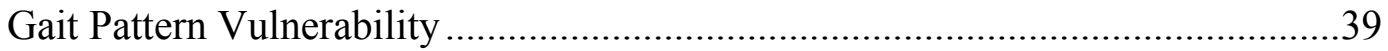

The Relationship Between Victimization History, Gait, and Personality ............40

The Relationship Between Victimization History, Gait, and Affect ....................41

The Effect of Time, Frequency, Severity, and Type of Victimization, on Gait ....42

Method

Participants 
Participant Pre-selection

Study 1: "The "Walkers"” participants

Measures and Equipment

Victimization History Questionnaire Extended .49

Revised Interpersonal Adjective Scales-Big Five .50 Manual for the Positive and Negative Affect Schedule Expanded Form .52 Kinematic Point-Light Display Technology.

Procedure 57

Results .59

Preliminary Data Screening .59

Primary Analyses 62

Discussion .126

Gait Pattern Vulnerability. 126

The Relationship Between Victimization History, Gait, and Personality

The Relationship Between Victimization History, Gait, and Affect

The Effect of Time, Frequency, Severity, and Type of Victimization, on Gait .135

Study 2: The Observers .142

Consensus 143

Accuracy

The Effect of the Observers' Perceptions of the Walkers' Personality and Affect on Ratings of Vulnerability 144

The Effect of the Walkers' own (Self-reported) Personality and Affect on

Likelihood to Experience Victimization 145 
Participants

Measures

Procedure

Results

Preliminary Data Screening

Primary Analyses

Discussion

Consensus

Accuracy

The Effect of the Observers' Perceptions of the Walkers' Personality and Affect

on Ratings of Vulnerability

The Effect of the Walkers' own (Self-reported) Personality and Affect on

Likelihood to Experience Victimization

General Discussion

Nonverbal Communication

A Victim Walk

Easy Prey: An Evolutionary Perspective

Kinematic Point-Light Display

Conclusion 
Appendix B: Mass Testing Section 1: Victimization History Questionnaire ...228

Appendix C: Study 1 \& Study 2: Demographic Questionnaire .229

Appendix D: Study 1: Victimization History Questionnaire Extended .230

Appendix E: Study 1: Recruitment Email .237

Appendix F: Study 1: Consent Form (deception) ......................................238

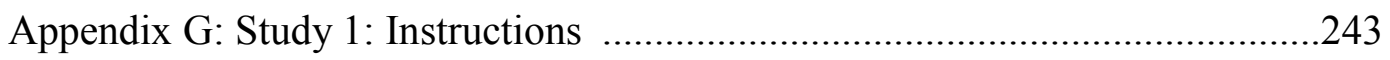

Appendix H: Study 1: Debriefing Form (reveals deception) ...........................244

Appendix I: Study 1: Consent Form (primary) ..........................................247

Appendix J: Study 1: Debriefing Form (final) .............................................251

Appendix K: Study 1: Mediation (Baron \& Kenny, 1986)...............................254

Appendix L: Study 2: Victim Vulnerability Rating Questionnaire ...................258

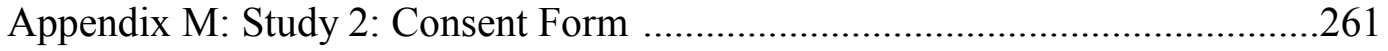

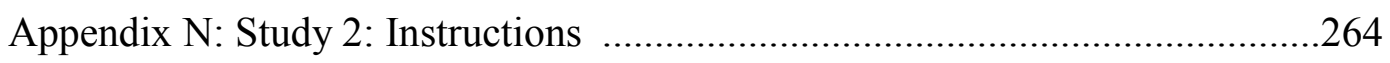

Appendix O: Study 2: Debriefing Form ..................................................265 


\section{List of Figures}

Figure 1: Side View Screen Capture of One of the Walkers

Figure 2: Mediation Model Examining the Relationship Between Victimization History,

Gait Pattern, and Personality .86

Figure 3: Relationship Between the Perceived Submissiveness of the Walkers and the Observers' Judgements of each Walkers' Vulnerability to Violent Victimization

Figure 4: Relationship Between the Perceived Submissiveness of the Walkers and the Observers' Judgements of each Walkers' Vulnerability to Sexual Victimization 


\section{List of Tables}

Table 1: Carleton's Motion Capture Laboratory Cameras .............................................55

Table 2: Descriptive Statistics for Participants with a Sexual Victimization History .......66

Table 3: Descriptive Statistics for Participants with a Violent Victimization History ......70

Table 4: Descriptive Statistics for the IASR-B5 Scales...............................................74

Table 5: Descriptive Statistics for the PANAS-X Scales .......................................... 77

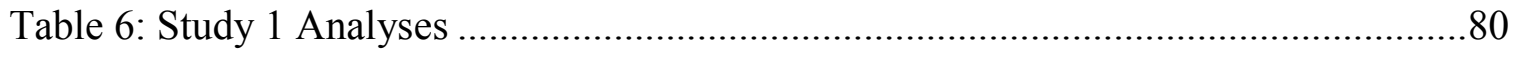

Table 7: Correlations Between Personality and Victimization History...........................83

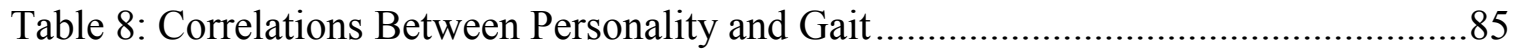

Table 9: Correlations Between Affect and Victimization History.................................91

Table 10: Correlations Between Affect and Gait..........................................................93

Table 11: Correlations Between Victimization Type and Personality...........................95

Table 12: Correlations Between Victimization Type and Affect ................................96

Table 13: Correlations Between Time Frame of Victimization and Personality..............99

Table 14: Correlations Between Time Frame of Victimization and Affect....................101

Table 15: Correlations Between the Frequency of Victimization and Personality..........103

Table 16: Correlations Between the Frequency of Victimization and Affect ................105

Table 17: Correlations Between Severity of Victimization Measured Nominally, and Gait

Table 18: Correlations Between Severity of Victimization Measured Nominally, and Personality. 109 
Table 19: Correlations Between Severity of Violent Victimization Measured Nominally, and Affect.

Table 20: Correlations Between Severity of Sexual Victimization Measured Nominally, and Affect.

Table 21: Correlations Between Severity of Victimization Measured on an Ordinal

Scale, and Gait

Table 22: Correlations Between Severity of Violent Victimization Measured on an

Ordinal Scale, and Personality

Table 23: Correlations Between Severity of Sexual Victimization Measured on an

Ordinal Scale, and Personality

Table 24: Correlations Between Severity of Violent Victimization Measured on an

Ordinal Scale, and Affect.

Table 25: Correlations Between Severity of Sexual Victimization Measured on an

Ordinal Scale, and Affect

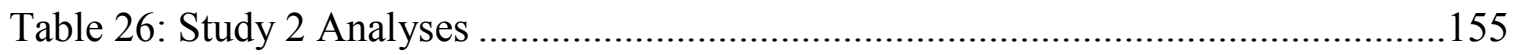

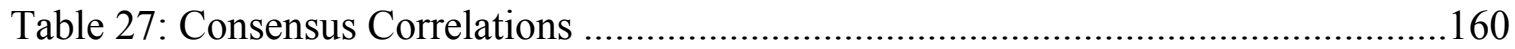

Table 28: Accuracy of Observer Ratings of the Walkers' Vulnerability to Violent

Victimization.

Table 29: Accuracy of Observer Ratings of the Walkers' Vulnerability to Sexual

Victimization. 163

Table 30: Self-other Agreement Correlations

Table 31: Chi-square Statistics Examining Differences in Rating Accuracy for Male and Female Observers. .170 
Walk This Way: A Kinematic Point-Light Investigation of Victim Vulnerability

"We respond to gestures with an extreme alertness and, one might also say, in accordance with an elaborate and secret code that is written nowhere, known to none, and understood by all.” (Sapir, 1949, p. 556)

Individuals can reliably ascertain numerous personality traits in others, including submissiveness and dominance, from nonverbal behaviour (Richards, Rollerson, \& Phillips, 1991). For example, the maintenance of an established posture and restrained gesturing reportedly indicate submissiveness; and research suggests that submissive body language implies vulnerability to others (e.g., Book, Costello, \& Camilleri, 2013; Richards et al., 1991). Alternatively, increased eye contact and a relaxed posture appear to cue more dominance (Richards et al., 1991; Simpson, Gangestad, \& Biek, 1993), and potentially less vulnerability. Nonverbal behaviour is used by others to guide their own subsequent behaviour (Mandal, 2014). For offenders, cues of vulnerability are of particular importance as they appear to guide victim selection (Book et al., 2013; Murzynski \& Degelman, 1996; Richards, 1991; Richards et al., 1991).

Research indicates that certain postures, gestures, and facial expressions contribute to the perception of vulnerability (e.g., Simpson et al., 1993). However, because isolation of nonverbal channels is difficult, the information communicated through discrete behaviours has rarely been systematically studied (Gunns, Johnston, \& Hudson, 2002). The specific behaviours that communicate vulnerability require attention. It has been argued that the delineation of gait is particularly important due to its ecological relevance (Sakaguchi \& Hasegawa, 2006). An individual's gait is one of the first behaviours that an observer has access to that can help them make judgements or 
form impressions about another person. It is therefore a key component in nonverbal communication between individuals. Recently, the perception of gait was found to be a cue in the prediction of vulnerability to victimization (i.e., individuals who indicate that they have been previously victimized tend to be chosen as potential future victims by others based on the way they walk; Book et al., 2013; Wheeler, Book, \& Costello, 2009).

The type of information attained through gait patterns may advance evolutionary theory (e.g., the ecological approach to perception; Montepare \& Zebrowitz-McArthur, 1988), and provide insight into spontaneous cognitive assessment processes. The study of gait movement (i.e., gait analysis) has also recently shown promise as an identification tool (Royal Canadian Mounted Police, 2014). For example, the analysis of a suspect's gait (e.g., recorded via surveillance cameras at the crime scene) has been used as expert evidence in both Canada and the UK. Gait analysis is likely to become increasingly useful in court proceedings (i.e., for identification purposes) as the type of characteristics that can be detected from gait become better understood. Furthermore, by determining the nonverbal cues that can be used in victim selection, a greater understanding of the "hunting process" of predatory offenders may be acquired; this allows for the development of targeted intervention strategies, which may help prevent future incidences of (often repeat) victimization.

Advances have been made in understanding how different types of offenders can detect cues of nonverbal vulnerability, and the extent to which detection ability depends on observer personality. For example, because psychopaths are characterized by their ability to manipulate and charm others, they tend to be adept at social predation and/or exploitation (Wheeler et al., 2009). It has been argued that a "successful opportunist" 
would be skilled in recognizing cues of vulnerability since targeting vulnerable individuals is likely to increase the chances of successful goal acquisition (i.e., sexual gratification, money, power, etc.). Recent research has revealed that individuals who score high on psychopathy do indeed appear to have an increased ability to recognize cues of vulnerability (i.e., identify "easy targets") via short observations of gait, relative to individuals lacking psychopathic traits (Book et al., 2013; Wheeler et al., 2009).

Unfortunately, however, significantly less attention has been paid to the traits of the walkers. Research suggests that targets may display "submissive-type" gait patterns and thereby indicate their vulnerability to others (e.g., Wheeler et al., 2009). However, there is yet to be an examination of whether or not previously victimized individuals with submissive personality traits actually exhibit the submissive (i.e., "victim walk") gait pattern. Other reasons (such as affect; e.g., Schneider, Christensen, Haubinger, \& Fallgatter, 2014) that may explain the differential gait patterns displayed by those who indicate a past victimization history, have not been considered. Furthermore, the association between accurate ratings of vulnerability (by observers) and the specific victimization experiences of walkers (e.g., violent or sexual) is unclear.

The purpose of Study 1 was to explore whether the association between victimization history and gait pattern could be accounted for by walker personality and/or affect. Given that there are confounds related to studying gait patterns (i.e., it is unclear if observers are making judgements based on gait alone, or other, related features such as age or attractiveness), kinematic point-light display technology was used to record individuals walking. Point-light display captures light-reflected body movement. The 
recording that is produced shows a moving lighted skeleton (with extraneous cues such as hair and weight, removed).

In Study 2, a sample of observers watched the point-light video recordings and rated the walkers on a number of characteristics including: vulnerability to future victimization, perceived submissiveness and dominance, and perceived positive and negative affect. The second study aimed to confirm (or disprove) previous links found between observer accuracy of walker vulnerability and victimization history. It also intended to provide a greater understanding of the type of information that observers can detect (e.g., cues of submissiveness) from short observations of body movement.

Following a review of nonverbal communication, the characteristics underlying a "victim walk" are considered. Gait as an information transmitter, and the adaptive ability of individuals to detect signals through gait, is discussed through the lens of evolutionary theory. The advantages of using kinematic point-light display technology to study human body movement (including gait) are then outlined. Nonverbal cues of submissiveness and personality traits inherent to victim selection are explored. Finally, the translation of affect into nonverbal behaviour are considered as a potential explanation for perceptions of vulnerability and victim recidivism. Research questions and hypotheses follow.

\section{Nonverbal Communication}

It has been estimated that approximately $30-35 \%$ of the social meaning in a conversation or interaction is translated verbally; nonverbal behaviour carries the heaviest weight (Birdwhistell, 1970). The 7-38-55 rule born from the early work of Mehrabian and colleagues (1967) asserts that in times of uncertainty (e.g., when attempting to interpret what another individual is expressing), we rely approximately $55 \%$ on facial 
cues, $38 \%$ on vocal elements, and only $7 \%$ on spoken words (also see Ehninger, Gronbeck, McKerrow, \& Monroe, 1986; Mehrabian \& Ferris, 1967; Mehrabian \& Wiener, 1967; Stewart \& D’ Angelo, 1988). The relative contribution of verbal versus nonverbal channels when individuals communicate information to others, and infer information about others, has become less central in linguistic and communication research (e.g., Graham, Unruh, \& Jennings, 1991; Hegstrom, 1979). However, the disproportionate influence of nonverbal behaviour on communication is widely recognized (e.g., Argyle, Alkema, \& Gilmour, 1971; Eskritt \& Lee, 2003). Traditionally, the definition of nonverbal behaviour has been broad enough as to include subtleties of speech such as speech pauses, speech intensity and speech duration (e.g., Crystal, 1969; Mehrabian, 1972). However, Mehrabian (1972) argues that the more accurate definition of nonverbal behaviour refers to any action distinct from speech. Therefore, postures, arm, leg and foot movements, facial expressions, and body movements are classified as nonverbal behaviours.

Ekman and Friesen (1969a) maintain that some nonverbal behaviours transmit information, whereas others are expressive or adaptive. By assuming movement is nonsporadic, it is possible to determine what specific movements (or combinations of movements) mean (Ritchie, 2014). Research has shown that various traits and emotions translate into nonverbal behaviours (e.g., Ekman, 1964; Ekman \& Friesen, 1965, 1968). For example, individuals with dominant personalities will inadvertently translate their authority through patterns of touch and less frequent smiling (Simpson et al., 1993). These behaviours are visible and thus easily detected by others. Others use the behaviours to assess an individual's state and intention(s); inferences are made regarding emotions, 
attitudes, interpersonal roles, personality, and severity of pathology, from nonverbal behaviour (Ekman \& Friesen, 1969a; Simpson et al., 1993).

Some researchers have found that individuals remain relatively accurate at judging the characteristics of others, even when the information has been degraded (e.g., Ambady, Conner, \& Hallahan, 1999; Ambady \& Rosenthal, 1992). For example, Stillman, Maner, and Baumeister (2010) found that stranger observers were able to accurately detect one's propensity for violence from brief glances (2-seconds) at photographs. Similarly, Fowler, Lilienfeld, and Patrick (2009) demonstrated that observers could accurately detect various features associated with psychopathy (e.g., callousness, manipulation, etc.; Brinke \& Porter, in press), via observation of "thin slices" of behaviour (5-second, 10-second, and 20-second video and/or audio segments).

Nonverbal behaviours do not only signal various traits and emotions to others, but also inspire action (e.g., approach or avoidance behaviour) in the observer. Simpson et al. (1993) explored personality and nonverbal behaviour during relationship initiation. A series of dyadic interactions were video recorded and observers $(n=210 ; 109$ females and 101 males) rated the recorded couples' nonverbal behaviours. Results suggested that men with an unrestricted socio-sexual orientation (i.e., those who engage in sexual relationships quickly, without concern of commitment or partner familiarity) were more likely to display nonverbal behaviours such as more smiling, less downward gazing, and more frequent flirtatious glances-behaviours indicative of non-dominance. These behavioural displays of affiliation communicate "contact-readiness" and are aimed at facilitating intimacy (Eibl-Eibesfeldt, 1989). Alternatively, women with a restricted socio-sexual orientation (i.e., those who require established relationships and tight 
emotional bonds prior to sexual intimacy) displayed fewer head cants and leaned away, relative to women with unrestricted socio-sexuality (Simpson et al., 1993). The nonverbal behaviour of the female has relevance for the male in that she is communicating her interest level, which simultaneously indicates whether he should initiate an approach.

Richards et al. (1991) examined the perceived submissiveness and dominance of women engaged in a video-recorded conversation with a confederate; four of the women recorded scored highly (30-37) on the dominance scale in the California Psychological Inventory (Gough, 1975), while the other four scored low on the dominance scale (1221). Approximately 32 male students rated the submissiveness and dominance of the women while watching the recordings on mute (i.e., the ratings were to be based solely on nonverbal body language). The results indicated that the observers could accurately differentiate the dominant women from the submissive women. The submissive women appeared to use less expansive movements, they maintained the same posture for extended lengths of time, and they gestured more often with their hands and feet. Those who had been rated as submissive were also subsequently judged as more likely to be a victim of sexual assault.

Simpson et al. (1993) argue that, similar to flirting behaviour, submissive behaviours imply "contact readiness" in relational communication. Submissive behaviours such as sidelong glances, weak smiles, and limited gesturing, are postulated to signal sexual interest and induce approach behaviour by another; these same behaviours also appear to suggest vulnerability. It is possible that nonverbal cues of submissiveness may unintentionally instigate approach behaviour in offenders during target selection. 
Nonverbal cues of vulnerability for offenders. Historically, much of the literature has differentiated between reactive and instrumental offenders (e.g., Blais, Solodukhin, \& Forth, 2014; Tapscott, Hancock, \& Hoaken, 2012). Reactive violent offenders are generally defined as those who act aggressively as a result of an extreme emotional response, often following a perceived provocation (Blais et al., 2014). They tend to appear impulsive, unreasonable, uncontrollable, and spontaneous in their actions (Tapscott et al., 2012). Instrumental violent offenders, on the other hand, engage in criminal activity in order to obtain an external goal (e.g., money, drugs, status, nonconsensual sex, etc.), and thus tend to act following premeditated and reasoned thought and planning; they are often referred to as "predatory," and their actions are calculated and deliberate (e.g., Blais et al., 2014). Tapscott et al. (2012) note that while reactive violence is an end in itself, instrumental violence is a means to an end.

Although recent research suggests that the dichotomy remains valid (i.e., it is possible to classify certain offenses into one of the two categories; Tapscott et al., 2012), perpetrators show much more overlap than previously believed. Offenders tend to have mixed motives for engaging in a crime, and they rarely engage in only one type of criminal activity (e.g., theft) throughout their entire career. For example, Tapscott et al. (2012) attempted to code nearly 200 offences from a violent adult male prison population in Ontario, Canada as either reactive or instrumental. They found that it was possible to code approximately 64 offenses (out of 188) as purely reactive, and 110 as purely instrumental (the remaining 14 were coded as a mixture of the two). Interestingly however, most, if not all, of the sample of offenders with a history of instrumentally violent crime, also had a history of reactive violence. Similarly, Blais et al.’s (2014) 
recent meta-analysis suggests that even psychopathic offenders, who are often assumed to be prototypical instrumental perpetrators, show considerable overlap in their offending: “...our results demonstrate equal relationships between psychopathy and instrumental and reactive violence across scale types" (p. 816).

Beauregard and Leclerc (2007) argue that both "types" of offenders (even those who have typically been described as irrational and/or impulsive [i.e., reactive type]), are capable of analyzing the costs and benefits of their actions. They conducted semistructured interviews with 69 male serial sex offenders whose crimes were committed against stranger victims. The offenders provided rationales for their actions during the pre-crime phase (e.g., pre-meditation, estimation of the risk of apprehension, forensic awareness, etc.), the crime phase (e.g., use/choice of weapon, restraints, etc.), and the post-crime phase (e.g., victim release site, etc.). Findings suggested that various factors, including certain victim behaviours, played a major role in the offenders' decisionmaking process.

Indeed, research indicates that offenders may consider geographic isolation (i.e., victim access), victim age/naivety, socioeconomic status of the neighborhood, and the routine activities of the victim (i.e., those who tend to engage in "risky" activities [e.g., they are visibly intoxicated, they post ads online, they are prostituting, etc.] appear to be targeted more often than those who do not engage in such activities; Beauregard, Rossmo, \& Proulx, 2007). The rational choice approach to crime argues that offenders decide to commit offences based on available, albeit limited information (i.e., rationality is "bounded" by time, information, knowledge and mental resources; Beauregard et al., 2007; Cornish \& Clarke, 1986; Snook \& Cullen, 2009). Offenders may decide their 
course of action by weighing the costs and benefits associated with the various methods available to them. Research indicates that cues of vulnerability are particularly important in the selection of victims for interpersonal crimes (e.g., mugging and sexual assault; Book et al., 2013; Selkin, 1975; Stevens, 1994; Wheeler et al., 2009). Targeting vulnerable individuals is likely to increase the chances of successful goal acquisition (i.e., sexual gratification, money, power, etc.), and thus becomes a crucial factor in the offender's decision-making framework.

A substantial body of research has been dedicated to the examination of situational and environmental factors that increase vulnerability (e.g., Beauregard et al., 2007; Cohen \& Cantor, 1981; Hough, 1987; Miethe \& Meier, 1990; Wright, Logie, \& Decker, 1995). However, the inherent attributes of individuals (such as body language), which also affect victim selection choice, have been understudied until recently. Myers et al. (1984) notes that individuals who report previous victimization tend to exhibit low dominance, low assertiveness and less social presence, relative to individuals without histories of assault. In stranger-observer studies, individuals can reliably report which targets display such characteristics and also tend to report these same individuals as those most likely to be re-victimized in the future (e.g., Book et al., 2013; Wheeler et al., 2009). In other words, individuals who exhibit submissive behaviours (i.e., those who cue vulnerability) tend to be selected as victims in these studies, whereby their enduring submissiveness elicits the possibility of future re-victimization.

Kilpatrick and Acierno (2003) argue that past victimization is highly predictive of future victimization (e.g., the risk of new assault for a previously assaulted individual is more than four times higher than someone without a history of assault; this risk appears 
to increase linearly where an individual's risk of new assault after having been previously assaulted twice is nearly $400 \%$ ). Thus, a small proportion of the population experiences disproportionate amounts of criminal victimization (Kilpatrick, Acierno, Resnick, Saunders, \& Best, 1997). Victim recidivism occurs across a range of crimes and “...repeat victims are often offended against by a number of different offenders..."

(Gunns et al., 2002, p. 130). Research suggests that past victimization may predict future risk because: 1) being victimized alters the individual in some way (e.g., individuals who are victimized may experience anxiety and/or depression, and research suggests that highly anxious individuals are at an increased risk of experiencing victimization; Lauritsen \& Quinet, 1995), or 2) because there is an unmeasured aspect of the victim that fosters their repeated selection by offenders (e.g., they exhibit risk-taking tendencies and/or work in a dangerous profession; Lauritsen \& Quinet, 1995; Sparks, 1981). Measures of vulnerability are particularly important as individuals may be unknowingly indicating a weakness to others through various attributes and behaviours (Gunns et al., 2002; Sparks, 1981).

Human perception studies have indicated that gait movements are particularly important for making judgements of others (e.g., Ikeda \& Watanabe, 2009; Schneider et al., 2014). These judgements are subsequently used as a cue for interaction. Gait is accessible to both strangers and known others. Therefore, the various gait patterns that signal and communicate characteristics to others are available to a wide audience. Recent research has demonstrated that specific gait patterns may indicate significantly more vulnerability relative to others (Book et al., 2013; Grayson \& Stein, 1981; Wheeler et al., 2009). Interestingly, victims of crime appear to exhibit the vulnerable gait pattern more 
often than individuals without histories of victimization (Wheeler et al., 2009). Therefore, it has been suggested that offenders may use gait as a target selection tool.

\section{A "Victim Walk?"}

Grayson and Stein (1981) examined differences in movement among individuals walking in a high assault area in New York City. The individuals (equal male/female split; $n=30$ males, $n=30$ females) were secretly video-taped to capture their natural gait patterns before they provided consent. Offenders ( $n=53$ male inmates) rated the assault potential of the various walkers and the walkers were divided into "victims" and "nonvictims" based on these ratings. The findings revealed that the gait patterns of victims were significantly different from the gait patterns of non-victims. Grayson and Stein (1981) identified five gait movement categories that differentiated the "victim walk" from the "non-victim walk:" Stride length, type of weight shift, body movement, type of walk, and feet. Victims, it seems, tend to have long or short strides, a lateral (i.e., weight shifts side to side), diagonal, or up/down shift movement, a gestural walk (i.e., movement activates only a part of the body [e.g., the legs]), unilateral arm/leg movements (i.e., antisynchronous movement; only one side of the body moves at a time), and lifted foot movements.

The gait that is produced is essentially inconsistent and awkward: "Non-victims have an organized quality about their body movements, and they function comfortably within the context of their own bodies. In contrast, the gestural movement of victims seems to communicate inconsistency and dissonance. Condon and Ogden (1966) have called this phenomenon "interactional synchrony"” (Grayson \& Stein, 1981, p. 74). 
A later study conducted by Murzynski and Degelman (1996) filmed three female confederates who were instructed to walk either as a "typical victim" (as defined by Grayson and Stein's [1981] movement categories [e.g., with lifted feet, unilateral arm/leg movements, etc.]), or as a non-victim. College students ( $n=41 ; 16$ male, 25 female $)$ and police officers ( $n=33,31$ male, 2 female) rated how vulnerable they believed the female confederates were to experience sexual assault. Their findings were consistent with Grayson and Stein's (1981) in that "typical victim" profiles were significantly more likely to be rated as vulnerable to sexual assault by both the students and the officers; those who walked as a "typical victim" were also judged to be significantly less confident relative to those who walked as a non-victim.

More recently, Sakaguchi and Hasegawa (2006) explored the gait patterns of female walkers and observer ratings of vulnerability to sexual exploitation. Walkers $(n=$ 15 female students) were asked about their victimization history in order to examine the accuracy of the vulnerability ratings. Similar to Grayson and Stein's (1981) “victim walk" prototype, females with shorter strides $(r=-.70)$ and slower gaits $(r=-.75)$ were rated (by 45 male students) as most vulnerable to sexual exploitation. However, the women who had reported past sexual exploitation were not the same individuals exhibiting the "victim walk," nor those chosen by the male raters as future victims.

Extending these findings, Wheeler et al. (2009) explored the relationship between ratings of vulnerability, psychopathy and previous victimization history. In order to capture the natural gait of walkers, 4 males and 8 female students were secretly filmed prior to providing consent. The walkers were then asked if they had ever been victimized and if they had, how many times. Victimization was defined as being equal to or greater 
than bullying. Approximately 2 males (out of 4) reported that they had been previously victimized, and 4 females (out of 8) reported a victimization history. The recorded walks were coded according to Grayson and Stein's (1981) movement categories and a total vulnerability score was assigned to each target. Male $(n=47)$ university students were recruited; they were asked to imagine themselves as a mugger and rate the targets on their vulnerability to victimization. The observers were also required to complete the SelfReport Psychopathy Scale: Version III (SRP-III; Paulhus, Neumann, \& Hare, in press), as it was hypothesized that higher psychopathy scores would be associated with greater accuracy (i.e., they would select individuals who had been previously victimized as those most vulnerable to future victimization).

Similar to other measures of psychopathy (e.g., the Psychopathy ChecklistRevised [PCL-R]; e.g., Hare, 1991, 2003; Hare \& Neumann, 2006, 2008; Neumann, Kosson, \& Salekin, 2007; Neumann, Vitacco, Hare, \& Wupperman, 2005; Vitacco, Neumann, \& Jackson, 2005), the SRP-III differentiates between the two major components of psychopathy, Factor 1 and Factor 2, as well as subdivides into four underlying facets: Affective, Interpersonal, Lifestyle, and Antisocial (e.g., Mahmut, Menictas, Stevenson, \& Homewood, 2011; Williams, Paulhus, \& Hare, 2007). Factor 1 assesses an individual's affective and interpersonal traits, whereas Factor 2 includes items related to an individual's lifestyle and their social deviancy. The underlying Affective facet pertains mainly to an individual's lack of empathy and remorse. The Interpersonal facet relates to glibness and grandiosity. The Lifestyle facet includes elements of impulsivity and sensation-seeking. Lastly, the Antisocial facet reflects the delinquency and criminal versatility features of psychopathy. 
Wheeler and colleagues (2009) found a significant correlation between body language and previous victimization (rho $(11)=.68)$. In other words, those scored as having high vulnerability on the movement categories were more likely to have selfreported previous victimization. Furthermore, there was a significant association between psychopathy scores and accuracy $(r=.38)$. Consistent with Sakaguchi and Hasegawa (2006), an observer was considered accurate if the walker that they rated as vulnerable to future victimization also had a history of past victimization. Individuals who scored higher on Factor 1 (i.e., the items which pertain to an individual's affective and interpersonal traits; $r=.43$ ), of the SRP-III, as well as the subscales Interpersonal ( $r=$ $.41)$, Affect $(r=.36)$, and Antisocial $(r=.29)$, rated target vulnerability more accurately compared to those with lower Factor 1 scores. The relationship between Factor 2 (i.e., the items related to an individual's lifestyle and social deviancy) of the SRP-III and accuracy was not significant.

Using the same video clips as Wheeler et al. (2009), Book and colleagues (2013) examined the accuracy of victim vulnerability ratings and psychopathy in a sample of inmates ( $n=47$ males). Inmates observed the videos of the targets walking and rated their propensity for getting mugged. They also completed the PCL-R (Hare, 2003). Total Psychopathy and Factor 1 scores were positively correlated with accurate ratings of victim vulnerability ( $r=.38$ and $r=.47$, respectively). The inmates were also required to provide a rationale for their ratings. Interestingly, unlike past studies (e.g., Amir, 1971; Wheeler et al., 2009), the inmates explicitly mentioned gait as a vulnerability cue. Although these findings require replication, it appears that extreme social predators (i.e., psychopaths) may have the ability 1) to accurately identify past victims as future victims 
from observation of their gait, and 2) to consciously recognize the cues of vulnerability that they attend to when selecting their target.

The reviewed research lends credence to Grayson and Stein's (1981) “victim walk" profile. However, there appears to be inconsistencies in the relationship between victimization history and accurate vulnerability ratings in samples of non-psychopathic observers. Further study is required to understand the association between victimization history and exhibition of the characteristic "victim walk."

\section{Easy Prey: An Evolutionary Perspective}

The communicative function of movement, particularly gait, may be better understood through the concept of evolutionary fitness. Nonverbal behaviours are mechanisms that evolved in our primate ancestors in order to communicate signals related to mating, hierarchy, competition and play (e.g., Tomasello, Call, Nagell, Olguin, $\&$ Carpenter, 1994). Similar to humans, non-human primates appear to exhibit specific nonverbal behaviours that communicate particular traits and intentions to others (Krauss, Chen, \& Chawla, 1996; Tomasello et al., 1996). For example, Tomasello et al. (1994) observed the gestures of captive chimpanzees-close primate relatives to humans. They observed that a ground-slap followed by throwing behaviour was repeatedly enacted to solicit play. The chimpanzees would then wait and watch the others in the group for a response. For primates, the ability to perform and decode nonverbal behaviour has serious implications for survival and reproduction. Interpreting a warning signal, deciphering approach signalling in a mate, or perceiving vulnerability in a rival, has repercussions at both inter and intra-species levels. 
For predatory mammals, vulnerability has consequences for prey selection. Animals who hunt tend to isolate and attack the most vulnerable in a flock or herd (Gunns et al., 2002). The concept of 'survival of the fittest' is based on the premise that the strongest, smartest, most environmentally adaptable individuals in a species will survive, reproduce, and pass their genes on to future generations (Darwin, 1869). In other words, the vulnerable members in a species will be naturally selected out. It is theorized that the detection of traits in others, such as vulnerability, evolved as a fitness mechanism (Schaller, 2008). Those able to detect and prey on vulnerable individuals are expected to be more successful in attaining food, territory, and status.

Gait is particularly important for the detection of vulnerability; health, age, injury and status can often be determined from gait cues. For example, many older mammals are slower and therefore tend to be preferentially selected by predators (Fanschawe \& Fitzgibbon, 1993): "Although the exact relationship between speed capacity and survival or reproductive success has seldom been tested empirically, locomotor speed is usually considered an 'ecologically relevant' trait” (Van Damme \& Van Dooren, 1999, p. 347). Some species will even feign gait injuries as a form of distraction display. The RedCrowned Crane, for example, will appear to limp in order to draw attention to themselves and away from their young (Wang, Zhou, \& Lu, 2013).

The ecological approach to perception has been used as a guiding framework for understanding why different gait patterns elicit equally different impressions in others (Gunns et al., 2002; McArthur \& Baron, 1983; Montepare \& Zebrowitz-McArthur, 1988). It suggests that characteristics that are observable (e.g., voice, movement, facial expressions) provide socially useful information (Montepare \& Zebrowitz-McArthur, 
1988); these characteristics indicate ways of acting and/or interacting with the individual. In other words, an observable characteristic (like gait) contains socially relevant information to observers, which signals how to interact with them. The recognition of socially relevant information, such as vulnerability, in others, is adaptive to one's own survival and goal attainment (Gunns et al., 2002). For example, the perceived sex of another occurs rapidly and accurately as sex has implications for one's own reproductive goals. Similarly, the vulnerability level of others is argued to be clearly specified in the social stimulus array and subsequently appraised because vulnerability originally had consequences for prey selection.

For humans, the production and interpretation of nonverbal behaviour(s) is adaptive for cueing friendship or affiliation, romantic interest, and danger, among others (e.g., Knapp, Hall, \& Horgan, 2013). The recognition of various traits in others through gait has likely been retained as well. It has been argued that offenders are modern-day predators, hunting for victims who offer adequate reward for minimal effort (Gunns et al., 2002). The reviewed literature suggests that there may be a distinct "victim walk" movement profile; the appraisal of which appears to be used as a selection cue for offenders (Grayson \& Stein, 1981).

Unfortunately, contemporary socially constructed cues (e.g., clothing) has confounded the study of purely biological gait patterns (potentially contributing to the inconsistent findings). It is also complicated to isolate gait from other body movement and qualities such as attractiveness, age, and sex, which makes firm conclusions difficult. "Biological motion capture" or "kinematic point-light displays" are useful for examining 
isolated movements, as they eliminate such confounds and can capture gait patterns independent of other cues (Runeson \& Frykholm, 1983).

\section{Kinematic Point-Light Display}

Point-light display was introduced into experimental psychology by Gunnar Johansson in the early 1970s (Johansson, 1973). The aim of the technology was to capture biological human body movement while removing extraneous cues (e.g., hair, weight, sex, clothing, etc.). The procedure has been altered over time and can vary depending on the availability of equipment. However, typically, reflective tape is attached to an individual's major joints (knees, elbows, ankles, etc.). A camera then records these individuals as they move in a darkened room. The recording that is produced shows a moving lighted skeleton. Point-light display has been used mainly to infer personality and demographic information from gait patterns. For example, it has been shown that individuals can reliably identify themselves (Beardsworth \& Buckner, 1981) and their friends (Cutting, 1977), age in strangers (Montepare \& ZebrowitzMcArthur, 1988), and dominance (Montepare \& Zebrowtiz, 1993), from short observations of point-light figures. As detailed below, kinematic point-light display has recently been used to examine the body movement of individuals who appear vulnerable to assault (Gunns et al., 2002).

The principle of kinematic specification of dynamics (KSD) argues contrary to beliefs that person perception occurs through ambiguous information channels and can be faked (Runeson \& Frykholm, 1983). KSD maintains that an organism's "dispositions" constrain and determine its kinematic (i.e., movement) pattern (Gunns et al., 2002; Runeson \& Frykholm, 1983). In humans, such dispositions include both mechanical 
properties (i.e., anatomical makeup), as well as internal states such as emotions and intentions (Runeson \& Frykholm, 1983). Species-specific information (actions, emotions, intentions, sex, identity, etc.) are therefore available to observers via movement patterns. Because the kinematic pattern is constrained by anatomical and internal states, the movement output represents genuine states of the individual. In line with the lawfulness of human movement, it is virtually impossible to perform truly deceptive movements.

Ekman and Friesen (1969a) claim that nonverbal behaviour(s) appear to "escape efforts to deceive" either by providing clues of the deceptive intention of the individual, or by "leaking" withheld information (p. 52). Ekman and Friesen noted differences in the face, hands and feet of deceptive individuals. They suggest that while some nonverbal behaviour can be censored, other behaviours cannot be easily controlled and may therefore unintentionally giveaway authentic internal information. Most individuals, they argue, rely on facial cues to judge whether or not someone is being honest - that which is most closely monitored and thus tends not to leak withheld information. Facial expressions can often be faked, "But the hands, unlike the face, are not fakers; most people will not use their hands to dissimulate... While facially smiling and pleasant [one] may be tearing at a fingernail, digging into his cheek, protectively holding his knees..." (p. 99). Similarly, the legs and feet, though easy to conceal under tables, are considered the worst "leakers" (i.e., they are the worst in terms of concealing deceptive information). Clues that suggest an individual may be attempting to hide something include aggressive foot kicks, tense leg positions, frequent shifting, and restless or repetitive movements.

Runeson and Frykholm (1983) argue that even "hidden" person properties related to personality and emotion are likely to be present in the kinematic pattern and can thus 
be detected from point-light images. Studies using kinematic point-light technology have shown that observers are impressively accurate at detecting various types of information from movement. For example, from only the lead-in movements of a person lifting a box, observers are able to determine the weight of the box (Runeson \& Frykholm, 1983). Furthermore, individuals cannot deceive observers about the weight of the box; rather, observers only detect that the individual is trying to deceive them. True conditions, it seems, are perceived by others despite the actor having deceptive intentions. Kinematic point-light technology has thus been used, fairly extensively, to obtain genuine information about individuals (e.g., Dittrich, Troscianko, Lea, \& Morgan, 1996; Hill \& Pollick, 2000; Hoenkamp, 1978; Johnston, Hudson, Richardson, Gunns, \& Garner, 2004; Pollick, Lestou, Ryu, \& Cho; 2002; Runeson \& Frykholm, 1983). In accordance with the KSD principle, it would seem that through gait, individuals are communicating genuine characteristics of themselves to others. The characteristics that generate perceptions of vulnerability to others (e.g., personality and affect) require investigation. Use of kinematic point-light display may help to both 1) validate notions that variations in biological gait patterns affect vulnerability ratings, and 2) explore the type of internal dispositional states that observers detect in order to infer vulnerability from gait cues.

\section{Investigating gait and victim vulnerability using point-light display. Using} point-light technology, Gunns et al. (2002) performed three experiments to investigate the association between gait and vulnerability to physical attack. As in previous studies, female walkers $(n=71)$ were recorded wearing light-reflectors in a darkened laboratory space; their movements were coded for vulnerability in accordance with Grayson and 
Stein's (1981) movement categories. A sample of male and female $(n=60 ; 30$ male and 30 female) observers rated the point-light targets on their vulnerability to experience mugging, as well as rape (Gunns et al., 2002). As expected, there was consensus on which walkers would be easiest to attack (ease-of-attack intra-class correlation $=.922$ ). Ratings were higher for the mugging crime, but participant ratings for both the mugging and rape questions were highly correlated $(r=.99)$. In addition, the walking patterns that had been coded as vulnerable were related to ease-of-attack ratings. There were no effects of observer demographics (e.g., sex) on ease-of-attack ratings. Therefore, it appears that "victim walks" and "non-victim walks" are possible to determine even from substantially degraded information.

The second study reported in Gunns et al.'s (2002) paper differed slightly in that only male walkers $(n=50)$ were used. Again, both male and female students $(n=60,30$ males and 30 females) rated the male walkers' vulnerability to physical attack. The results were very similar to those obtained for the female sample of walkers: There was consensus among observers regarding which male walkers would be easiest to attack (ease-of-attack intra-class correlation $=.920)$. Likewise, the "vulnerable" coded walks were significantly correlated with ease-of-attack ratings. More specifically, walkers with a gestural gait, shorter stride lengths (relative to their height), and more constrained walks (among other features), were rated as easiest-to-attack. Again, there was no effect of rater demographics on vulnerability ratings. Thus, it appears that the relationship between walking style and ease-of-attack ratings also exists for male walkers.

Lastly, Gunns et al. (2002) argued that because gait movement does not occur in a vacuum it is important to investigate the effect of additional information on perceptions 
of vulnerability. They explored the effect of clothing and footwear on vulnerability ratings. For the clothing condition, the point-light display of 30 female walkers was altered to show them wearing either leggings, trousers, or a skirt. For the shoes condition, walkers were shown wearing high heels, flat shoes, or bare-feet. Interestingly, differences in clothing and footwear resulted in different walking styles.

Approximately 36 males and 36 females rated the walkers on their vulnerability to future victimization. The differences in gait following the introduction of shoes and clothing predicted differences in vulnerability ratings. For example, individuals wearing the skirt had significantly shorter stride lengths relative to their height, they lifted their feet more and they had a slower walking speed. As a result, ratings of vulnerability changed; those in the skirt condition were rated as easier to attack compared to individuals wearing either the leggings or the trousers. Again, the demographics of the observers did not have an effect on their ratings.

Because gait patterns changed when individuals wore different clothing, Gunns et al. (2002) surmised that cognitive states may have a profound effect on an individual's nonverbal behaviour. For example, it has been postulated that different clothing styles may alter feelings of power in individuals, consequently affecting their outward behaviour (Gunns et al., 2002; Henley, 1977). Given the differential ratings following the addition of clothing and shoes, Gunns et al. (2002) notes that “...the vulnerability of any given individual is not, therefore, fixed but can vary as a function of factors such as attire" (p. 154-155).

Alternatively, however, an early study by Montepare and Zebrowitz-McArthur (1988) found no significant differences between vulnerability ratings for individuals in 
point-light conditions and individuals in full view conditions. They concluded that regardless of other nonverbal cues such as attractiveness or body shape, body language is a salient factor in the perception of others. Historically, gait has been a reliable signal for inherent traits; appearance cues such as clothing and shoes may be socially constructed. It is possible that genuine traits are represented in gait movement prior to becoming confused with the addition of compound appearance cues. Overall, the few studies that have utilized point-light technology have rendered mixed findings. Additional research exploring the communicative function of isolated gait movements is required.

Furthermore, thorough background information on walker individuals is necessary, as the association between genuine characteristics of the walker (e.g., personality traits) and vulnerability ratings is unclear.

\section{Personality and Victim Selection}

Personality can be characterized as a set of psychological traits and information processing mechanisms that are organized and enduring (Larsen \& Buss, 2008). An individual's personality affects how they engage with and respond to their environment. Interpersonal theory maintains that an individual's personality is shaped by the relationships in their life (Sullivan, 1953). Personality is, therefore, formed by others and subsequently affects how one relates to others. The "interpersonal domain" is a popular area of study for personality theorists who examine the relational aspect of personality formation. The interpersonal domain consists of a number of variables, which interrelate in a two-dimensional space known as the "circumplex" (Acton \& Revelle, 2002). The interpersonal circumplex was developed in part to elucidate the relational factors of 
human personality. It helps organize and evaluate relational behaviours, traits and motives (Gurtman, 2009; Locke, 2006; Markey \& Markey, 2009; Wiggins, 2003).

The circumplex is designed as a circle split into quarters (Gurtman, 2009). Two quarters are defined by "agency." Agency typically includes concepts related to power, dominance, status and control. The other two quarters are defined by "communion." Communion is comprised of variables such as love, affiliation, union and friendliness. The broad concepts of agency and communion underpin an individual's interactions with others. Essentially, individuals either "get along" (communion), or "get ahead" (agency). Coordinates designate the major interpersonal personality traits, each of which is a specific "blend" of communion and agency. On opposite poles of the circle (90 degrees and 270 degrees) is assured-dominant and unassured-submissive, respectively. Warmagreeable and cold-hearted define the two horizontal poles. The remaining angles include: gregarious-extraverted, aloof-introverted, arrogant-calculating, and unassumingingenuous (Trapnell \& Wiggins, 1990). The octants that are closest together are positively related, whereas opposing octants are negatively related (Markey \& Markey, 2009). The model makes a number of assumptions; for example, it assumes that all of the interpersonal personality variables are equally relevant, and that the circle is inclusive and exhaustive of all possible interpersonal traits (Gurtman, 2009).

The interpersonal circumplex is arguably the most popular model used to examine the dominance-submissive dyad (Markey \& Markey, 2009), and it has been used to explore other issues relevant to forensic psychology (e.g., it has been used as a model to study the victim-offender relationship in sexual assault crimes; Alison \& Stein, 2001; Porter \& Alison, 2004). In general, dominance refers to the tendency to behave in 
assertive, forceful, and self-assured ways (Wiggins, 1979). Individuals high in trait dominance tend to emerge as social leaders (Lord, De Vader, \& Alliger, 1986), they speak more, control group processes and decisions (Judge, Bono, Illies, \& Gerhardt, 2002), and exude competence and expertise regardless of their actual ability (Anderson \& Kilduff, 2009). Submissive personality traits, on the other hand, refer to the tendency to behave in helpless, appeasing, and fearful ways (Carli, LaFleur, \& Loeber, 1995).

Empirical investigation of the detection of personality traits from nonverbal behaviour began in the early 1900s (e.g., Wolff, 1943). Many of the nonverbal behaviours that individuals display, including gait, seem to suggest either that the individual has a submissive personality or, alternatively, a dominant personality (Book et al., 2013; Carli et al., 1995; Richards et al., 1991; Simpson et al., 1993). However, assessing an individual's submissiveness relative to their dominance is difficult. While some researchers argue that dominance is its own trait and based on a continuum ranging from high to low (e.g., Cattell's Sixteen Personality Factors; Cattell, 1946), others use dominance, status, and competence interchangeably (Carli et al., 1995). The lack of definitional coherence has contributed to measurement scales that do not explicitly measure dominance, but rather measure "correlates" of dominance such as ambition or assertiveness (e.g., Hogan Personality Inventory, NEO Personality Inventory; Costa \& McCrae, 1985; Hogan 1986). Similarly, submissiveness is often measured as a component of "interpersonal sensitivity" or "compliance." One of the few tools that measures submissiveness and dominance as their own constructs is the Interpersonal Adjective Scales (IAS; Wiggins, 1995). 
The IAS, originally created by Wiggins (1979), appears to be the current measure of choice to evaluate the dominance-submissive dyad, as well as other interpersonal personality traits present in the circumplex model (Gurtman, 2009). The original Interpersonal Adjective Scales (IAS) consisted of 128 items, but was later reduced to 64 in the revised version (IAS-R). The revision of the original IAS to a shorter version improved its substantive and structural characteristics, while maintaining acceptable reliability (i.e., Cronbach's alpha ranged between $.749-.857$ in nine test samples; reliability decreased only $\alpha=.045$ from the original IAS; Wiggins, Trapnell, $\&$ Phillips, 1988). The IAS-R consists of eight scales, one for each interpersonal trait: warmagreeable, gregarious-extraverted, assured-dominant, arrogant-calculating, cold-hearted, aloof-introverted, unassured-submissive, and unassuming-ingenuous. Recently, associations have been drawn between the IAS-R and the Five-Factor Model.

The Five-Factor Model of Personality (i.e., the "Big Five") is a well-established taxonomy of the five basic dimensions of personality (openness, conscientiousness, extraversion, agreeableness, and neuroticism; McCrae \& Costa, 1989). It has been argued that the Five-Factor Model and the circumplex are complementary models of personality (McCrae \& Costa, 1989; Trapnell \& Wiggins, 1990). While the Five-Factor Model provides a framework that helps to orient and understand the circumplex, the circumplex details and elaborates on the relational traits in the Five-Factor Model (McCrae \& Costa, 1989; Trapnell \& Wiggins, 1990). The "Big Five" factors correspond with many of the traits in the circumplex; for example, extraversion and agreeableness are intrinsically interpersonal and are related to dominance and submissiveness (also referred to as "nurturance") in the circumplex. A 124-item combination tool, the IASR-B5, which 
includes both circumplex traits and "Big Five" personality characteristics, has been developed and is considered to be a reasonably sound duel assessment tool. The IASR-B5 makes it possible to evaluate the submissiveness versus dominance of an individual while also examining the broader, related concepts in the Five-Factor Model.

The International Personality Item Pool - Interpersonal Circumplex (IPIP-IPC) is an open source assessment tool that has also been developed from the IAS-R (Markey \& Markey, 2009). It is shorter (32-items) and arguably easier for participants to understand. Although a glossary is provided with the IAS-R to assist participants who may have difficulty understanding certain adjectives on the scale (e.g., boastless), Markey and Markey (2009) argue that the IPIP-IPC is even further simplified for participants. It has demonstrated overlap with the IAS-R and has retained the circumplex structure. It is argued to be a valid measure for quickly assessing interpersonal circumplex traits (Markey \& Markey, 2009).

The IAS, its successors, and the IPIP-IPC are all unique measures of personality; they assess characteristics that affect an individual's relationship with others. Within the reviewed literature, it is argued that dominance and submissiveness (key components of the circumplex model; Markey \& Markey, 2009; Simpson et al., 1993), can be detected from nonverbal behaviour with a high degree of accuracy (e.g., Richards et al., 1991; Simpson et al., 1993), largely because they have increased implications for observers (Simpson et al., 1993). It has been suggested that submissive individuals may be perceived by others as vulnerable (Book et al., 2013; Richards et al., 1991). If a submissive personality is characterized by behaviours that indicate helplessness and the 
appeasement of others, it is not surprising that this type of personality would connote a vulnerability to others.

Victimology studies have shown that individuals who self-report that they are shy and less confident tend to be targeted by offenders (Sakaguchi \& Hasegawa, 2006). Other research has suggested that offenders are adept at detecting vulnerability in nonverbal behaviour (specifically gait patterns; Book et al., 2013) because it increases the chances of successful goal acquisition. Thus, it is possible that individuals with submissive personality traits may be inadvertently translating their submissiveness through their gait, thereby cueing vulnerability. In other words, offenders may be able to distinguish submissive-trait individuals from dominant-trait individuals through the observation of gait patterns, and will subsequently select the submissive individuals to victimize (e.g., Sakaguchi \& Hasegawa, 2006). However, because little attention has been paid to the personality of chosen victims, it is unclear if individuals who self-report as submissive actually exhibit the "victim walk." In one of the only studies investigating this relationship, Sakaguchi and Hasegawa (2006) found mixed support for the association between target choice and self-reported personality traits of walkers.

Using point-light display, female targets were recorded while walking (Sakaguchi \& Hasegawa, 2006). Male students rated the likelihood of the targets being approached for a certain kind of advance (e.g., inappropriate touching). The walkers reported the actual frequency that they have been approached, and several personality traits were examined. In terms of personality, the walkers who were rated as likely victims by the observers rated themselves as neurotic, shy, low in self-monitoring and introvertedcorrelates of submissiveness. Accuracy between the observer ratings and self-reported 
approach frequency of the walkers was also analysed. Although there was consensus among the observers regarding which point-light walkers were most vulnerable to each advance, the observer ratings of vulnerability did not correlate with the targets' reported advance experience(s). In other words, from an observation of gait patterns, observers selected the same target for exploitation. However, observers did not choose those individuals who had indicated that they had been previously victimized as those who would be revictimized in the future. Overall, the association between the walkers' selfreported personality traits, victimization history, and accuracy of observer ratings was partially supported.

Yeagley, Morling, and Nelson (2007) argue that distinguishing between accuracy and consensus is required if one wants to determine if observers are truly detecting individuals with submissive personalities. The distinction between these concepts is also important for examining if observers are truly detecting past victims as future victims. Accuracy is achieved through "self-other agreement" (i.e., questioning the targets on their own personality traits or victimization history, and subsequently correlating these selfreports with the observer ratings), whereas consensus is simply the rate of agreement among observers.

Recently, Yeagley et al. (2007) investigated the accuracy of undergraduate judges in predicting self-esteem, social dominance orientation, satisfaction with life, extraversion, masculinity and femininity, following 30s silent video clips. Mixed samples of male and female judges observed two same-sex friends involved in a muted conversation. The judges either watched clips of six female targets, or six male targets conversing (but not both). There was observer consensus for male targets on all items 
(i.e., self-esteem, social dominance orientation, satisfaction with life, extraversion, masculinity, and femininity). However, these ratings were only accurate (i.e., correlated with the video-recorded individuals' self-report) for satisfaction with life, extraversion, and masculinity, in males. Similarly, for female targets, though there was observer consensus on all the items, observers only attained self-other agreement on extraversion.

Yeagley et al. (2007) argue that the lack of concordance between self and observer reports for female targets may be a result of the affordances (e.g., social context) present. For example, social dominance orientation and masculinity may be easier to diagnose by others when males are engaged with other males. Greater agreement may have occurred for the female targets if measures of affiliation or interdependence had been included; it is suggested that these traits are likely to be more salient during interactions between females, and would have been more recognizable to others. Yeagley et al.'s (2007) findings emphasize the importance of accounting for gender in zeroacquaintance research, as well as the need for distinct measurements of consensus and agreement.

Given the limited research that has been conducted in the area, it is necessary to explore the relationship between the self-reported personality of walkers, the pattern of their walk, and observer vulnerability ratings. It appears that there may be a discrepancy between observer accuracy and observer consensus in ratings of victim vulnerability. Therefore, it is necessary to disentangle the two concepts and examine them individually. However, it is also possible that the vulnerability observers detect is not the nonverbal "output" of a submissive personality at all. Research indicates that affective states also translate into gait patterns and can be detected by observers (Schneider, Christensen, 
Haubinger, Fallgatter, Giese, \& Ehlis, 2014). It is possible that affect (e.g., sadness), as opposed to personality traits (e.g., submissiveness) connote the vulnerable gait patterns observed in victims.

\section{Affect and Victim Selection}

It is generally accepted that internal emotional states are outwardly displayed in body language (e.g., Ekman, 1972; Ekman \& Friesen, 1967; Karg, Kuhnlenz, \& Buss, 2010; Montepare, Goldstein, \& Clausen, 1987). For example, disgust is often expressed with a nose wrinkle and anger by a furrowed brow and tightened mouth (Matsumoto, Keltner, Shiota, O’Sullivan, \& Frank, 2008). Some evolutionary psychologists theorize that outward expressions of emotion occur because they are elements of coordinated physiological response systems. The body language that expresses anger occurs in the mouth and forehead because these are constituents of the attack response. Emotions of disgust are expressed in body language such as the nose wrinkle because it is part of the vomiting response. It appears that, consciously or not, emotion "leaks" into body language. Research indicates that the recognition of emotional displays in others is also biological and argued to be adaptive (Van den Stock, Righart, \& de Gelder, 2007). Indeed, impaired emotion recognition is symptomatic of various psychiatric disorders (e.g., personality disorders), and is the central deficit in Autism Spectrum Disorder (Schneider et al., 2014).

An early study by Ekman and Friesen (1967) explored participants' ability to determine emotions from various head and body cues. Participants observed a set of 60 still photographs; 30 of which had a face (head cue) and 30 of which were of a headless body (body cue). Agreement was higher for head cues relative to body cues $(z=3.24)$, 
however there was no consistent pattern observed between the agreement on one cue and agreement on the other. In other words, the emotion perceived from body cues was unrelated to the emotion perceived from head cues. In light of the inconsistent findings, Ekman and Friesen (1967) hypothesized that body positions in and of themselves may convey little information; whereas body movement, on the other hand, is likely to communicate more information about specific emotions.

Subsequent studies have corroborated this argument; body movement appears to relay various emotions to others (e.g., Frijda, 2010; Karg et al., 2010; Montepare et al., 1987). Gesture frequency, body leaning, and posture changes have been delineated for the purposes of understanding their affective meaning. However, relative to other nonverbal movement behaviour, gait has been understudied as a transmitter of affective information (Montepare et al., 1987). Recent research suggests that variations in gait may convey distinctly different emotions to others (e.g., Schneider et al., 2014). For example, Ikeda and Watanabe (2009) investigated emotion detection accuracy for point-light display walkers. Observers were able to determine the affect of walker individuals at better than chance levels; accuracy was highest for angry walks as opposed to happy or neutral walks. Schneider et al. (2014) argues that, "Human gait reflects a specific type of dynamic body motion which provides sufficient information for the perception of expressed motivational or emotional states" (p. 381). Schneider and colleagues (2014) expanded on Ikeda and Watanabe's (2009) findings by including fearful and sad pointlight display walkers in their study. They then examined the detection accuracy of observers while simultaneously recording their neurophysiological changes. 
Functional near-infrared spectroscopy (FNIRS) was used to monitor the brain changes of observer individuals ( $n=33 ; 10$ males and 23 females) while they processed and rated the emotional states of faceless avatars (Schneider et al., 2014). The avatars had been developed by recording 13 lay theatre actors who had been instructed to reflect on past life events in order to prompt a specific emotion (anger, fear, happiness or sadness). The actors were told to avoid gesturing and walk straight. Speed was statistically controlled for in order to assess emotion detection when gait speed was held constant.

FNIRS results showed significant activation in the right extrastriate body area (EBA), right inferior temporal gyrus (ITG), and left temporo-parietal junction (TPJ) when participants observed sad, fearful and angry walks. Less activity was observed in these areas when participants observed neutral or happy walks. Angry and fearful walks also activated the left posterior superior temporal sulcus (STS). Oxygenated cortical haemoglobin $\left(\mathrm{O}_{2} \mathrm{Hb}\right)$ increased for sad, angry and fearful walks relative to neutral walks. However, peak $\mathrm{O}_{2} \mathrm{Hb}$ concentrations in the left posterior superior temporal sulcus (pSTS) were recorded when participants observed the sad and fearful avatars walking. Overall, these findings indicate that emotion recognition accuracy was highest for the avatars displaying sad and fearful gait patterns, with sad walks having the highest accuracy ratings (i.e., sad walks were the most easily recognizable), and producing the highest arousal.

Contrary to research suggesting that positive nonverbal behaviours (e.g., smiling) provokes approach behaviour from others, new research argues for the alternative. Schneider et al. (2014) suggest that recognition, accuracy, and arousal, may be increased for negative emotions because they are more likely to affect the observer: "In contrast to 
sad, fearful and angry body movements, the observation of happy walks may initiate less personal involvement due to their, compared with negative walks, reduced personal relevance and implications for the observer's behaviour" (p. 387). Biologically, negative emotions have increased consequences for observers (Schneider et al., 2014). Perceived sadness, for example, is likely to trigger a secondary emotional reaction such as empathy, in an observer. Similarly, observing anger in someone else may be associated with an activation of one's own defense system. Positive and neutral emotional displays in others are arguably less relevant for observers (e.g., a happy individual has little to no relevance in terms of one's own survival).

Outward displays are used by others to formulate an impression of the affective state of another person (Asch, 1946). Impression formation is important for observers as it helps to guide their own subsequent behaviour. Schneider et al. (2014) suggests that there is a direct link between perceiving another individual's emotional state and one's own action and motivation. Some researchers go so far as to define emotions as processes that cause actions, autonomic changes, and feelings in oneself and in others (e.g., Frijda, 2010). Frijda (2010) argues that the intimate relationship between emotion and action can occur immediately, or can develop over time. For example, animals will cry or whimper when they are hurt. It has been suggested that such event sequences are an automatic or “impulsive action response" to an emotion. Alternatively, emotions can be enduring; at times producing intentional actions. Domestic disputes, abuse, and murder, are actions often committed out of prolonged anger and/or hatred. In other words, the appraisal of situations, events, and/or emotions in another person appears to elicit particular emotions and subsequent actions from oneself. 
Nonverbal displays of fear and sadness are postulated to implicate vulnerability to others. Because emotion can be defined by its connection to action in oneself and in others, it is possible that "vulnerable" emotional displays in individuals elicit subsequent emotions and actions (e.g., approach behaviour) in offenders. Quick and accurate detection of these states would provide an indication of individuals who are "easy prey." Furthermore, the reported short- and long-term negative effects on victims of crime may help explain why individuals with victimization histories appear to walk differently than others. Research suggests that individuals who have been victimized tend to experience sadness (depression), anger, and fearful affective states (Kilpatrick, Saunders, AmickMcMullan, Best, Veronen, \& Resnick, 1989; Wasserman \& Ellis, 2007). The reviewed literature suggests that, like others, victims may outwardly express such states in their body language, including in their gait pattern. The negative affect produced by the victimization history (and subsequently expressed in gait) may unintentionally cue outside individuals with malicious intent, leading to a cycle of victim recidivism wherein an individual is repeatedly offended against. The triplicate relationship between victimization history, gait pattern, and affect, has not yet been examined. To do so, the affect of individuals who report a victimization history would need to be investigated prior to studying their gait pattern for vulnerability.

The affective state of individuals is often measured using the Manual for the Positive and Negative Affect Schedule (PANAS; Watson, Clark, \& Tellegen, 1988). The two factors, positive affect and negative affect, have consistently emerged as the major dimensions of emotional experience both cross-culturally and across time (Almagor \& Ben-Porath, 1989; Diener, Larsen, Levine, \& Emmons, 1985; Watson \& Clark, 1994; 
Watson et al., 1988). The PANAS includes two 10-item scales, one to assess general positive affect and one to assess general negative affect. The PANAS was expanded to become the PANAS-X. The PANAS-X is a 60 -item version that includes higher order scales including fear, sadness, guilt, and hostility, among others.

An additional version referred to as the International Positive and Negative Affect Schedule Short Form (I-PANAS-SF) was also created, mainly for culturally diverse samples (Thompson, 2007). The I-PANAS-SF is shorter (10 items in total) compared to both the original PANAS as well as the PANAS-X and argued to be less redundant for non-English speaking individuals. However, because the PANAS-X is exhaustive and provides an involved assessment of an individual's affective state on higher order variables, it tends to be the method of choice (Watson \& Clark, 1994). The measurement of affect in individuals with victimization histories may provide a foundation for the subsequent study of their gait pattern(s).

\section{Study 1: The "Walkers"}

Advances have been made in determining the personality traits of observer individuals, as well as the specific gait pattern that generates ratings of vulnerability. For example, research has investigated whether or not individuals who score higher on psychopathy provide more accurate ratings of victim vulnerability (i.e., if they choose past victims as future victims based on gait information; e.g., Book et al., 2013; Ritchie, 2014; Wheeler et al., 2009). However, very little research has investigated the "walkers" themselves. The above review suggests that individuals may exhibit distinct nonverbal behaviours that indicate their vulnerability to others. It is currently assumed that cues of submissiveness translate into gait and are subsequently detected by observers. 
Unfortunately, this assumption is circuitous - do all submissive individuals display the “victim walk?" If not, why might some submissive individuals exhibit their vulnerability through gait while other submissive individuals do not?

A comprehensive understanding of the chosen targets is warranted. It is possible, for example, that affect, as opposed to personality, is being translated into gait. There is considerable research suggesting that there is a biological translation of emotions such as sadness, anger and fear into nonverbal behaviour (e.g., Ekman \& Friesen, 1967). Schneider et al. (2014) argues that the perception of negative emotions in others is more likely to increase approach and interaction behaviour, potentially for criminal purposes. It is possible that individuals with victimization histories may have increased negative affect, which becomes present in their walking, and is in turn detected by observers. Furthermore, the specific victimization history of the walker including the type of victimization that they have experienced, the frequency with which they have experienced victimization, the period of time that the victimization took place in, and the subjective severity of the event, requires examination. Categorization and analysis of victimization history may confirm previous hypotheses, and provide additional targetspecific information.

The aim of Study 1 was to empirically investigate the personality, affect, and victimization history of walkers. Male victims have been largely ignored in the current literature, and yet account for nearly half (48.8\%) of all victims of violent crime (Truman, 2011). Thus, the current study expanded upon previous research by including male as well as female walkers. Furthermore, a control group of individuals who indicated that they had never been victimized were included so that comparisons with 
self-reported victims could be carried out. In order to examine the relationship between self-reported personality (e.g., submissiveness) and affect (e.g., sadness) with gait, participants were recorded using kinematic point-light display technology. Researchers coded and scored each participants' walk according to Grayson and Stein's (1981) movement categories, thereby distinguishing them in to "vulnerable" or "non-vulnerable" categories. It was then possible to investigate whether individuals with vulnerable "victim walks" were the same individuals who reported a victimization history, had submissive personality traits, and/or conveyed higher negative affect.

Gait pattern vulnerability. Research indicates that past victims display a vulnerable gait pattern relative to others without victimization histories (e.g., Book et al., 2013). Five movement categories have been used to distinguish victims from nonvictims: 1) stride length, 2) type of weight shift (i.e., transfer of weight from one foot to the other), 3) body movement, 4) type of walk, and 5) foot movement (Grayson \& Stein, 1981). A “typical” victim, according to Grayson and Stein's (1981) movement categories, would exhibit a long or short stride (not a medium stride), shift their body weight laterally, diagonally, or with an up/down movement (not three-dimensionally), walk gesturally (not posturally), move unilaterally (i.e., one side at a time) as opposed to contralaterally, and lift their feet (not swing them; see Appendix A). The present study sought to replicate the relationship between victimization history and the exhibition of vulnerable gait characteristics (according to the aforementioned parameters).

$\boldsymbol{H}_{1}$. It was hypothesized that the gait patterns of individuals who indicated that they had been previously victimized would map onto the characteristic "victim walk" 
pattern (i.e., individuals who had been victimized would exhibit more of the vulnerable features [described above] in their gait).

The relationship between victimization history, gait, and personality. To date, the literature has suggested that individuals with vulnerable gait patterns are inherently more submissive and thus more likely to experience victim recidivism (i.e., an individual's submissiveness may be expressed through their gait). However, this relationship has not been delineated. Therefore, analyses were conducted to explore the relationship between victimization history, gait, and personality.

$\boldsymbol{H}_{2 .}$ A strong, positive correlation was expected between vulnerable-coded gait patterns and the Unassured-Submissive scale in the IASR-B5. In other words, it was predicted that individuals who exhibited vulnerable gait cues would also report submissive personality traits. On the other hand, a statistically weaker correlation (relative to that between vulnerable-coded gaits and Unassured-Submissiveness) was expected between vulnerable-coded gait patterns and the Assured-Dominant scale in the IASR-B5.

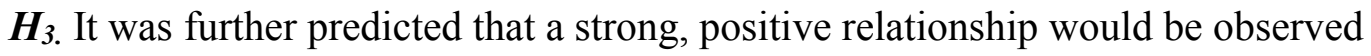
between the Unassured-Submissive scale in the IASR-B5 and victimization history. More specifically, it was expected that individuals who had been victimized would be more likely to self-report submissive personality characteristics. It was predicted that a statistically weaker correlation (relative to that between victimization history and the Unassured-Submissive scale) would be observed between those who had a victimization history and the Assured-Dominant scale in the IASR-B5. 
$\boldsymbol{H}_{4}$. Lastly, it was hypothesized that personality (namely submissiveness) would act as a partial mediator between victimization history and gait. In other words, it was predicted that a submissive personality would (partially) explain why those who have been victimized appear to exhibit vulnerable gait cues.

The relationship between victimization history, gait, and affect. The reviewed research has indicated that affect is expressed through one's movement, and that negative affect in particular, may be perceived of as vulnerable to others. However, the current thesis is the first known to explore the relationship between victimization history, gait, and affect.

$\boldsymbol{H}_{5 .}$ A strong, positive correlation was expected between vulnerable-coded gait patterns and the General Negative Affect scale in the PANAS-X. Strong, positive correlations were also expected between vulnerable-coded gait patterns and the higherorder Fear, Sadness, and Hostility scales in the PANAS-X. In other words, it was predicted that individuals who exhibited vulnerable gait cues would also report negative affective states. Statistically weaker correlations (relative to that between vulnerablecoded gaits and General Negative Affect) were expected between vulnerable-coded gait patterns and the positive PANAS-X scales.

$\boldsymbol{H}_{6 .}$ It was further predicted that a strong, positive correlation would be observed between victimization history and the General Negative Affect scale in the PANAS-X. More specifically, it was expected that individuals who had been victimized would be more likely to report greater negative affect. Strong, positive correlations were also expected between those who had a victimization history and the higher-order Fear, Sadness, and Hostility scales in the PANAS-X. It was predicted that statistically weaker 
correlations (relative to that between victimization history and General Negative Affect) would be observed between those who had a victimization history and the positive PANAS-X scales.

$\boldsymbol{H}_{7 .}$ Lastly, it was expected that affect (namely negative affect) would act as a partial mediator between victimization history and gait pattern. In other words, it was predicted that negative affect would (partially) explain why those who have been victimized appear to exhibit vulnerable gait cues.

The effect of time, frequency, severity, and type of victimization, on gait. Research has yet to thoroughly explore the victimization history of the walkers, and the relationship between victimization history and gait patterns. Preliminary research exploring the type of victimization that the walkers have experienced has found that observers may be proficient at identifying those with violent and sexual victimization histories relative to those who have been violated less interpersonally (e.g., Selkin, 1975; Stevens, 1994; Wheeler et al., 2009). However, the definitions of sexual and violent victimization have been varied and show arguable overlap. Therefore, the present study examined the type of victimization individuals report in greater detail.

In addition, there is yet to be an exploration investigating the effect of time of victimization on vulnerability (as expressed through gait). It is possible that victimization which occurred long ago, relative to recently, has less of an effect on body language cues. Similarly, long-term victimization as opposed to an isolated incident may alter body language. The severity of the victimization experience, where severity is defined subjectively, may also impact body language. For example, an instance of workplace harassment may be perceived of as fairly insignificant by some individuals, and thus it 
may not impact their body language in the same way that it would for an individual who perceived their experience of workplace harassment to be very disturbing or damaging. Therefore, the present study performed a descriptive analyses of the effect of time, victimization frequency, and severity, on gait. Given the lack of previous research examining the effect of specific victimization experiences, on gait cues, no formal hypotheses were made.

\section{Method}

\section{Participants}

Participant pre-selection. Mass testing allows researchers to administer short (approximately 10 minute) measures. The measures may be designed to pre-select for certain characteristics, they may include scales that must be administered in a session separate from the main study to reduce demand characteristics, and/or they can ensure a specific target sample actually exists in the student population. A pool of participants that have the required characteristic(s) is produced and these individuals can then be later invited to participate in the main study. Given that the present thesis was partially exploratory, mass testing was conducted to: 1) ensure that the type of individuals required for the studies truly exist (in robust numbers) within the student population (i.e., students that have been victimized in various ways), and 2) acquire a pool of individuals with the necessary characteristics (i.e., students with victimization histories) that could be later invited to participate in Study 1.

A mass call went out to all incoming first year (and various second year) undergraduate psychology students at Carleton University. Students were invited to 
participate in a series of on-line questionnaires, ${ }^{1}$ their participation was entirely voluntary and there was no compensation provided to those who participated. The entire mass testing package was approved by the Carleton Ethics Committee for Psychological Research (REB \# 14-127).

Participants began the mass testing questionnaire by providing consent and moving through the various (randomly ordered) pre-selection surveys. The "Walker Preselection Survey" included two sections. Section 1 was designed to capture individuals who had experienced various types of victimization (i.e., violent, sexual, and/or nonviolent; see Appendix B). Participants simply indicated (by checking "yes" or "no") whether they had experienced each type of victimization.

Because certain personality features (namely submissiveness) were expected to account for the relationship between victimization history and gait pattern, Section 2 of the mass testing package included a short 32-item questionnaire, the IPIP-IPC (Markey \& Markey, 2009), which measured the participants' interpersonal personality features. The IPIP-IPC adhered to mass testing time constraints and included statements such as, "am quiet around strangers," "cut others to pieces," and "talk to a lot of different people at parties." Participants rated themselves on a scale ranging from 1 (very inaccurate) to 5 (very accurate), to indicate the degree to which each statement accurately described themselves.

In development and testing, the IPIP-IPC maintained psychometric validity and retained the circumplex structure of the IAS-R, from which it was based (Markey \& Markey, 2009). However, largely because of its brevity, the reliability of the IPIP-IPC's

\footnotetext{
${ }^{1}$ There were numerous other pre-selection surveys included within the mass testing package (e.g., from social psychology, developmental psychology, etc.); the "Walker Pre-Selection Survey" was only one of them.
} 
four-item octant scales are slightly lower than that of the IAS-R's (Markey \& Markey, 2009). Furthermore, because it was developed fairly recently, re-test consistency has not been confirmed (Markey \& Markey, 2009). Nevertheless, Markey and Markey (2009) argue that it is useful for brief, time restricted assessments and for individuals who may have difficulty understanding certain terminology used in the IAS-R. Upon completion (or withdrawal) from the questionnaire, participants were directed to an online debriefing form.

A total of 1397 undergraduate students completed the "Walker Pre-selection Survey." Approximately 51.8\% $(n=720)$ of individuals reported that they had experienced some type of victimization (violent, sexual, and/or non-violent). More specifically, $24.2 \%(n=338)$ indicated that they had been non-violently victimized (e.g., break and enter), 15\% $(n=207)$ indicated that they had been sexually victimized (e.g., rape), and $12.6 \%(n=175)$ indicated that they had been violently victimized (e.g., mugging). ${ }^{2}$ The results suggested that over half of students in a randomly selected sample, will self-report that they have experienced some form of victimization.

In relation to the IPIP-IPC, the majority of participants $(n=855,61.2 \%)$, scored highly (i.e., scored 4 [moderately accurate] or 5 [very accurate]) on behaviours that indicated a warm-agreeable disposition. A lesser number scored highly on the gregariousextraverted scale $(n=311,22.3 \%)$, the unassuming-ingenuous scale $(n=203,14.5 \%)$, the aloof-introverted scale $(n=111,7.9 \%)$, the unassured-submissive scale $(n=111$, $7.9 \%)$, the assured-dominant scale $(n=45,3.2 \%)$, the arrogant-calculating scale $(n=19$,

\footnotetext{
${ }^{2}$ Nation-wide victimization surveys do not always report the frequency rates of each type of victimization (i.e., violent, sexual, non-violent) experienced by college/university students. Therefore, it is unclear how normative the current rates are. However, recent North American estimates suggest that the rates obtained may be similar to those found elsewhere (e.g., Sinozich and Langton [2014] note that approximately 20\% of female students report [to the police] that they have been a victim of rape and/or sexual assault).
} 
$1.4 \%)$, and the cold-hearted scale ( $n=17,1.2 \%)$. The results suggested that there was moderate variability among the different scales, which would allow for the mediated regression between victimization history, personality, and gait (in Study 1), to be conducted.

To determine the variability of personality attributes in individuals with victimization histories specifically, those who self-reported that they had been previously victimized (violently, sexually, and/or non-violently) and scored highly (i.e., they indicated that the behaviour described was "moderately accurate" or "very accurate" of themselves) on the various personality scales, were analyzed. Once again, there was appropriate variation among the items. For example, some individuals with victimization histories scored highly on the warm-agreeable scale $(n=319,23 \%)$, while others scored highly on the gregarious-extraverted scale $(n=126,9.1 \%)$, the unassuming-ingenuous scale $(n=76,5.4 \%)$, the unassured-submissive scale $(n=46,3.3 \%)$, the aloof-introverted scale $(n=39,2.8 \%)$, the assured-dominant scale $(n=19,1.4 \%)$, the arrogant-calculating scale $(n=10, .7 \%)$, and/or on the cold-hearted scale $(n=7, .5 \%)$. These results suggested that certain personality traits (e.g., submissiveness) and victimization history do co-vary in a student population. 
Victimization history was used as a preselection criterion for Study 1; students with a violent and/or sexual victimization history, ${ }^{3}$ who provided their contact information, were emailed an invite asking them to partake in a movement study. Interested individuals could sign-up for a time-slot through Carleton's research participation network (i.e., SONA). A random sample of students without victimization histories was also recruited in order to obtain a control group.

Personality, on the other hand, was not used to pre-select for Study 1. The mass tested pool of participants reported a variety of personality characteristics. Therefore, it was feasible to simply invite those with a victimization history and obtain a sample with a variety of personality features. Although affect was also expected to account for the relationship between victimization history and gait pattern, affect was not assessed in mass testing because measures that attempt to gain an overall indication of one's affect (e.g., the PANAS-X) were too lengthy to include in the mass testing package. Given the typical undergraduate affective norms reported in other research (e.g., Watson \& Clark, 1994), it was expected that the invited sample of students would report a variety of affective states.

\footnotetext{
${ }^{3}$ Non-violent victimization can include a variety of events. Therefore, not surprisingly, a high number of individuals reported that they had been non-violently victimized $(n=338)$ in the mass testing survey. Many of the victimization events were possibly minor (e.g., stolen phone), and given previous discussions in the area (e.g., Selkin, 1975; Stevens, 1994), it was unlikely that individuals who had experienced non-violent victimization would display vulnerability (via their gait) to the same extent that someone who had been victimized more interpersonally would. Given time, technological, and resource constraints, it was important to ensure that participants recruited for the current studies provided meaningful reports of prior victimization. Therefore, non-violent victimization was disregarded in Study 1; differences in gait were only examined for those with a violent victimization history and/or for those who had experienced sexual victimization.
} 
Study 1: "The 'Walkers"” participants. ${ }^{4}$ The sample for Study 1 consisted of 31 students from Carleton University. Each session took approximately one hour to complete; all participants enrolled in an introductory psychology course were provided $1 \%$ extra credit as compensation for participating. Approximately $67.7 \%(n=21)$ of the sample was female and $32.3 \%(n=10)$ was male. Participants ranged in age from 18 to 32 years $(M=20.03, S D=2.89) .{ }^{5}$ There were no significant differences in age between males $(M=20.60, S D=2.07)$, and females $(M=19.76, S D=3.22) ; t(29)=-.75, p=$ .460. The majority of participants $(71 \%, n=22)$ were Caucasian, $12.9 \%(n=4)$ were Asian, 6.5\% $(n=2)$ were African Canadian/Black, 3.2\% $(n=1)$ were First Nations/Aboriginal Canadian/Native Canadian, and 6.5\% $(n=2)$ designated themselves as from another unspecified ethnic background. There was no significant difference observed between the number of Caucasian males who participated $(36.4 \%, n=8, z=.3)$ and the number of Caucasian females who participated $(63.6 \%, n=14, z=-.2), \chi^{2}(4)=$ $1.98, p=.739 .^{6}$

Approximately $58.1 \%(n=18)$ of the sample indicated that they had been violently and/or sexually victimized. There was no significant difference between the number of males who reported a victimization history $(70 \%, n=7, z=.5)$, and the number of females who reported one $(52.4 \%, n=11, z=-.3), \chi^{2}(1)=.86, p=.353$; more detailed information related to the victimized cohort is presented below. The remaining $41.9 \%(n=13)$ of the sample reported that they had not been victimized violently or

\footnotetext{
${ }^{4}$ A sample size of 40 undergraduate students was estimated apriori. Power was calculated by conducting a compromise power analysis using G*Power (Faul, Erdfelder, Buchner, \& Lang, 2009). A one-tailed, correlational (Point-biserial) model, with an effect size set at .3 (medium; Cohen, 1992) projected a power of $84 \%$. Thus, the smaller sample of 31 that was obtained was slightly underpowered.

${ }^{5}$ The study was restricted to those of legal age (i.e., 18 years of age or older) to provide consent.

${ }^{6}$ All other categories of ethnicity had cell counts of less than 5; therefore, chi-square was not conducted on these items.
} 
sexually; this set acted as the control group. No other identifying information was collected from the participants.

\section{Measures and Equipment}

Upon arriving at Carleton's Motion Capture Laboratory (located in the School of Information Technology within the Azrieli Building), each participant received an experimental package to be completed following the point-light recording session. The package contained a demographic questionnaire (comprised of three questions that asked participants to indicate their age, gender, and racial/ethnic background; see Appendix C), a victimization history questionnaire (see Appendix D), as well as two scales: the IASRB5 and the PANAS-X. The questionnaires/scales contained in each package were ordered randomly to control for potential order effects.

Victimization History Questionnaire Extended. The Victimization History Questionnaire is a short, self-report survey that was originally developed and piloted with an undergraduate student population at Brock University (Wheeler et al., 2009). It asked students to report whether or not they had ever been victimized (yes/no), and if they had been victimized, the number of times. The survey was extended in an undergraduate thesis at Carleton University (Ritchie, 2014). The extended survey asked if the participant had ever experienced violent victimization, sexual victimization, or any type of victimization in a yes/no format (these same questions were present in the Walker Preselection Survey). It was adapted for the current study such that if the participant responded positively to any of the victimization questions, then they were directed to answer eleven further questions; one question asked them to indicate the frequency with which they had experienced victimization, one question asked them to report how long 
ago the victimization event had occurred, and nine of the questions pertained to the subjective impact/influence that the experience had on them (see Appendix D).

Given the difficulty in measuring subjective severity in a brief self-report type

format, four of the nine impact/influence questions were adapted from the Brief Trauma

Questionnaire (Schnurr, Spiro, Vielhauer, Findler, \& Hamblen, 2002) and the Trauma

History Screen (Carlson et al., 2011); both have been used to assess clinical in-patient

populations, and non-referred community and student samples. The questions asked

participants if they believed that they were in danger at the time of the event, whether or

not they were seriously injured, how bothered by the experience they were, and about

their feelings at the time of the event. Five broader questions (created by the researchers),

questioned the individual's distress following their victimization, the impact that the

experience had on them, and their perceived emotional, psychological, and physical

severity of the event. The Victimization History Questionnaire Extended included a short glossary to help participants discriminate between sexual and violent victimization, ${ }^{7}$ if necessary.

Revised Interpersonal Adjective Scales-Big Five. The IASR-B5 is a 124-item questionnaire that aims to assess both circumplex traits, as well as "Big Five" personality characteristics (Trapnell \& Wiggins, 1990). It is touted to provide an indication of an

\footnotetext{
${ }^{7}$ It should be noted that the definitions of "sexual victimization" and "violent victimization" that were used in the mass testing survey during the recruitment process of Study 1, were different from the definitions that were used in Study 1's "Victimization History Questionnaire Extended." Upon further examination (post mass testing), it became apparent that certain terminology used in the mass testing definitions may be problematic. For example, "stalking" was included as an example of "sexual victimization." However, stalking could technically also be considered a violent or non-violent crime. The definitions were thus revised in the "Victimization History Questionnaire Extended" in an attempt to ameliorate the issues. The new definitions were printed on the front page of the "Victimization History Questionnaire Extended" and participants were instructed to read them carefully. Therefore, participants who may have previously categorized their victimization as a certain type in the mass testing survey had the opportunity to revise their answers based on the new definitions. Nevertheless, it is possible that the inconsistency impacted the type of sample that was recruited for Study 1.
} 
individual's dominance and submissiveness (i.e., nurturance)—coordinates inherent to the interpersonal circumplex-while also providing global markers of neuroticism, conscientiousness, and openness to experience, from the Five-Factor Model. The FiveFactor model also contains a surgency/extraversion dimension and an agreeableness dimension. However, because surgency/extraversion and agreeableness from the "Big Five" appear to be variants of the dominance-submissiveness dyad in the circumplex, they are combined together in the IASR-B5 and do not have their own scales (Trapnell \& Wiggins, 1990; Wiggins et al., 1988).

The IASR-B5 is composed of eight scales or "octants;" the octants correspond to the eight interpersonal circumplex coordinates (assured-dominant [PA], gregariousextraverted [NO], warm-agreeable [LM], unassuming-ingenuous [JK], unassuredsubmissive $[\mathrm{HI}]$, aloof-introverted [FG], cold-hearted [DE], and arrogant-calculating [BC]). Each octant consists of eight personal adjectives. For example, items 7 and 9 on the warm-agreeable (LM) octant are "soft-hearted" and "kind," respectively. The FiveFactor dimensions (conscientiousness [CONSC], neuroticism [NEUR], and openness to experience [OPNS]), also have their own scales, each of which are comprised of 20 adjectives. Individuals are required to self-report on the adjectives (ranging from $1=$ extremely inaccurate to $8=$ extremely accurate), the degree to which they describe their own characteristics. The octants and each Five-Factor domain scale is scored by obtaining the mean of the relevant adjective ratings that comprise it. An individual's interpersonal circumplex traits and "Big Five" characteristics can then be compared and contrasted to one another. 
According to Trapnell and Wiggins (1990), the IASR-B5 “...was found to have excellent structure on the item level, internally consistent scales, and promising convergent and discriminant properties when compared with the NEO Personality Inventory and the Hogan Personality Inventory" (p. 781). They argue that because the IASR-B5 contains both Five-Factor features as well as interpersonal trait information, researchers can obtain global markers of populations, as well as additional (and more specific) information that would allow one to differentiate between populations.

Therefore, the IASR-B5 appears to be particularly well-suited for mass screening, for classifying individuals into typological categories, and for those investigating new domains (Trapnell \& Wiggins, 1990).

Manual for the Positive and Negative Affect Schedule Expanded Form. The original PANAS contains two, largely uncorrelated (ranging from $r=-.12$ to -.23 when examined across various test samples with differing time instructions; see below), 10 item scales; one scale assesses positive affect and one assesses negative affect (Watson et al., 1988). In development and testing, the PANAS scales have demonstrated that they have high internal consistency, and exhibit quasi-independence across student, adult, and clinical in-patient populations (e.g., Watson et al., 1998; Watson \& Clark, 1994). The PANAS asks participants to rate (on a 5-point scale), the degree to which they have experienced various mood states (e.g., excited). When tested across different sets of temporal instructions (e.g., "Indicate to what extent you have felt this way right now," versus "today," versus "during the past few days," versus "during the past week," versus "during the past few weeks," versus "during the past year," versus "in general, that is, on 
average") the measure has remained stable (i.e., the reliability of the scales ranges from Cronbach's $a=.84$ to .90 and are not affected by the time instructions).

In 1994, the PANAS was expanded to become the PANAS-X (Watson \& Clark, 1994). The PANAS-X includes 13 scales, 11 of which are higher-order scales. The general dimension scales remain as positive affect and negative affect. However, the PANAS-X also includes a set of basic negative emotion scales (inclusive of fear, hostility, guilt and sadness), a set of basic positive emotion scales (inclusive of joviality, self-assurance, and attentiveness), and a set of "other" affective state scales (inclusive of shyness, fatigue, serenity and surprise). The item composition varies for each scale. For example, the "fear" scale has six accompanying adjectives: afraid, scared, frightened, nervous, jittery, and shaky; whereas the "surprise" scale has three adjectives: amazed, surprised, and astonished. The PANAS-X contains 60 items in total; Study 1 utilized the PANAS-X in order to obtain the most inclusive account of each walker's affect (particularly their fear, hostility, and sadness which were expected to influence their gait pattern).

Similar to the PANAS, the PANAS-X instructs participants to rate (using a scale ranging from $1=$ very slightly or not at all to $5=$ extremely), the extent to which they have felt each adjective over a specified period of time (e.g., "today"). The current study instructed participants to report the extent to which they felt a certain emotion (e.g., fear) “during the past few weeks." Ratings of longer time periods (such as "during the past few weeks" or "during the past year") appear to result in implicit aggregations (Watson et al., 1988). In other words, when individuals are asked to reflect on an extended time frame, they tend to average their responses across time and events. Watson et al. (1988) have 
suggested that these aggregated responses provide a trait measure of affect. Obtaining an indication of an individual's overall or "typical" affect is important for the current study as general affect may be argued to influence ingrained movement cues more than fluctuating mood states would.

The PANAS-X takes participants an average of 10 minutes to complete and it is relatively simple to administer and score (responses in each scale are summed for a total scale score). In independent testing, it has maintained high internal consistency reliabilities and it is regarded as a psychometrically sound tool to measure positive and negative affect, in addition to higher order affective states (Watson \& Clark, 1994).

Kinematic Point-Light Display Technology. Study 1 took place in Carleton's Motion Capture Laboratory, which is located in The School of Information Technology (CSIT). Associate Professor, Dr. Anthony Whitehead, and a trained technician from the CSIT department agreed to help run participants through the point-light display system. The laboratory itself is a large $27^{\prime}$ X $38^{\prime}$ rectangular room (Joslin, 2010). The oversized area allows for optimal recording of moving targets and ensures that the reflective markers are detected. The space was darkened in order to prevent the images from becoming obscured from outside infrared light. All reflective surfaces were removed to ensure the cameras captured only target-specific information.

The motion capture system consists of 10 mounted Vicon MX40 cameras; each camera contains a strobe and a specialized filter. The strobe appears as intense red lights that circle in front of the camera. The cameras illuminate the reflective markers on the suit of the walking target. The filter is used to screen out wavelengths that are outside the range of $623 \mathrm{~nm}$ of "visible" strobe. The recording that is produced appears as grayscale 
light. Detailed camera sensor information, pixel size, and lens specifications are outlined in Table 1 below.

Table 1

Carleton's Motion Capture Laboratory Cameras

\begin{tabular}{ll}
\hline Components & \multicolumn{1}{c}{ Specifications } \\
\hline Camera Type & Vicon MX40 \\
Sensor Type & CMOS \\
Sensor Resolution & $2352(\mathrm{~W}) \times 1728(\mathrm{H})=4,064,256$ pixels \\
Sensor Depth & $10 \mathrm{bits} /$ pixel grayscale; 1024 gray levels \\
Sensor Dimensions & $16.46 \mathrm{~mm}(\mathrm{~W}) \times 12.10(\mathrm{H}) ; 20.43 \mathrm{~mm}$ diagonal \\
Pixel Size & 7 microns x 7 microns (square pixels) \\
Focal Length & $12.5 \mathrm{~mm}$ \\
\hline
\end{tabular}

Light reflective tape is commonly wrapped around an individual's major joints to produce the kinematic image. However, tape and/or glue tends to be inconvenient and can lack precision. Therefore, participants in the current study were asked to wear a black Vicon suit. The Vicon suit used in the Motion Capture Laboratory has adhesive properties (it is made of Velcro-hoop material), which allow retro-reflective globe-shaped markers to be attached using Velcro-hook pads. For the purposes of the current experiment, $18 \mathrm{~mm}$ markers were. These markers are ideal for body tracking because they are soft, flexible and can be easily detected by the cameras.

Though isolated kinematic movement is important to obtain, recent research has suggested that individuals may alter their gait when they believe that they are being watched and/or when their movements are being directly attended to (e.g., A. Book, personal communication, May 7, 2015; Johnston, Hudson, Richardson, Gunns, \& Garner, 2004). Therefore, once a participant was suited up and calibrated to the cameras, they were told to engage in a number of menial tasks as a means to distract them from their 
walking behaviour. For example, they were instructed to watch the researcher complete a zigzag pattern around the room, and were then requested to retrace this pattern on their own (i.e., as a faux memory test). The cameras filmed the entire process and stopped once the CSIT technician indicated that a satisfactory gait sequence had been captured. This standardized process ensured that: 1) all walkers walked in the same direction (for at least a portion of the recording), 2) that a useable sequence of gait could be captured, and 3) that participants walked as naturally as possible (i.e., by engaging in the memory exercise they were not directly attending to their walking behaviour). The Vicon Blade software was used to edit the videos.

The video footage was edited in order to ensure that each walker appeared clearly as a point-light figure (a screen capture of one of the walkers viewed from the side is presented in Figure 1); each video was also reduced to an approximately 10 second clip (consistent with Book et al., 2013; Ritchie, 2014; and Wheeler et al., 2009). Videos that appeared damaged and/or were unreadable due to camera glare, reflection, or calibration error, were discarded. Three of the 31 videos could not be saved and were expunged from the system; the sample size decreased to 28 walkers. ${ }^{8}$

\footnotetext{
${ }^{8}$ All survey data was analyzed using the total 31 participants; only the analyses requiring gait statistics used the smaller sample of 28.
} 


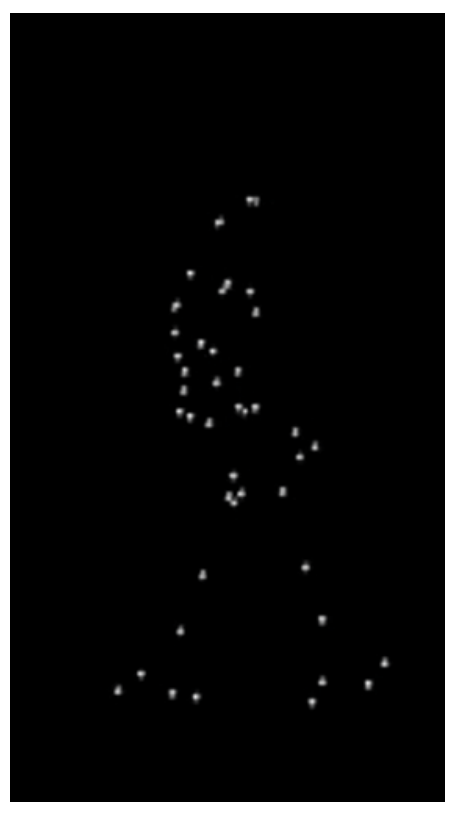

Figure 1. Side view screen capture of one of the walkers

\section{Procedure}

Students with a violent and/or sexual victimization history, who provided their contact information, were invited to participate in Study 1. A random cohort of students without victimization histories were also invited in order to obtain a control group. The standardized email invite (see Appendix E) asked students to participate in a study examining the association between memory and movement. The email contained a brief summary informing participants that they would be asked to change into a tight black suit, walk around a room whilst being videotaped, and provide information related to their victimization history. Interested individuals could sign-up for an available time-slot through Carleton's research participation network (i.e., SONA). The entire study took each participant approximately one hour to complete and participants were compensated 
for their time by receiving one study credit (1\%), which was allocated to one of their introductory psychology courses. ${ }^{9}$

Upon arrival, participants were presented with a consent form outlining the possible risks and benefits of taking part in the study (see Appendix F). All students consented and were asked to change into a black Vicon suit. As in Gunns et al.'s (2002) study, participants were reassured that the recorded images would be unrecognizable; they could observe a video clip of someone else (one of the researchers) walking in the suit in order to demonstrate that only a moving lighted image would be produced by the cameras.

Once participants had been calibrated to the cameras, they were told that recording had begun. They were asked to watch as the researcher walked in a zig-zag pattern (which they were told to remember as a faux memory exercise). They were then told to retrace the pattern themselves, and to walk off the set once they were done. The memory task was included in order to elicit the most natural gait possible from the participants (i.e., to distract them from focusing on their movement). Once the CSIT technician confirmed that an acceptable gait sequence had been captured, participants were told that the recording had finished and they were invited to change back into their regular clothing. The walking portion (part 1) of the study took each participant approximately 30 minutes to complete.

In part 2 of the study, participants completed their study package, which included a set of instructions (Appendix G), as well as four questionnaire sections. One section contained the short demographic questionnaire requesting participant age, gender, and

\footnotetext{
${ }^{9}$ Approximately six participants had already fulfilled their study credit maximum and/or simply requested to volunteer their time.
} 
ethnicity. Another section contained the Victimization History Questionnaire Extended, which aimed to assess the type, frequency, time-frame, and subjective severity of victimization that the individual had experienced. Participants also completed the IASRB5, which measured the participant's personality, with a particular emphasis on the submissiveness-dominance dyad. Lastly, the PANAS-X was completed; the PANAS-X is a measure of general participant affect. All sections were ordered randomly across participants to control for potential order effects. The complete package took participants approximately 20-25 minutes to finish.

Upon completion, participants were provided a debriefing form that explained the full purpose of the study and outlined how they had been deceived (i.e., since the study was portrayed to be related to memory and movement; see Appendix H). Following their debriefing, participants received a second consent form (Appendix I), which outlined how their data could be used in the future, and requested the release of the participant's data for usage. All participants were thanked for their time and provided with a final debriefing form (Appendix J). The entire Study 1 package was approved by the Carleton Ethics Committee for Psychological Research (REB \#15-057).

\section{Results}

\section{Preliminary Data Screening}

Missing data. Poor camera capture resulted in the loss of 3 walker videos. Therefore, while survey data was present for all 31 participants, only 28 of the recorded video clips were useable. There was no missing data observed for any of the survey questions (i.e., all 31 participants completed the entire demographic questionnaire, Victimization History Questionnaire Extended, IASR-B5, and PANAS-X). 


\section{Outliers.}

Categorical variables. There were thirteen categorical variables in total: Gender, ethnicity, victimization history (yes/no response for sexual and violent victimization), distress related to victimization (yes/no response for sexual and violent victimization), feelings of danger during victimization (yes/no response for sexual and violent victimization), injury as a result of victimization (yes/no response for sexual and violent victimization), and feelings of fear during victimization (yes/no response for sexual and violent victimization). Minimum and maximum values were used to screen for outliers; there were no out-of-range data entry errors observed.

Continuous and ordinal variables. Ordinal variables included: the frequency and timing of one's victimization (sexual and violent), the length of time and the extent to which one was bothered by their victimization (sexual and violent), the emotional, psychological and physical severity of victimization (sexual and violent), and the PANAS-X and IASR-B5 scale items. Participant age was the only continuous variable. All items were first assessed for outliers via visual inspection of box-plots; a number of potential outliers were noted. The variables of concern were then further examined by calculating their z-scores. Given the small sample size $(n<100)$, z-scores \pm 2.50 were deemed inappropriate (Van Selst \& Jolicoeur, 1994).

One participant exceeded the cut-off value on the Fear $(z=3.62)$, Guilt $(z=2.67)$, Hostility $(z=2.95)$, and Shyness $(z=3.17)$ scales of the PANAS-X. Similarly, one participant had an outlying score on the Assured-Dominant $(z=2.63)$ and WarmAgreeable $(z=-2.72)$ scales of the IASR-B5. There were no outliers observed on any of the ordinal items in the Victimization History Questionnaire Extended. The data from the 
participants with the outlying scale scores were re-examined for data entry errors; no corrections were required. Because the outliers were extreme but valid scores, and the analyses to be conducted were robust to violations, they remained as is in the dataset.

Normality. The main analyses conducted in Study 1 did not require that the variables be normally distributed (i.e., normality need not be assumed for ordinal regression, the Mann-Whitney U test, etc.). However, several correlations among the scale and composite variables required the use of parametric data. Therefore, all ordinal variables were assessed for normality via visual inspection of histograms and box-plots. In addition, skewness and kurtosis was calculated for all of the PANAS-X and IASR-B5 scales, as well as for the ordinal victimization variables. Certain items, particularly those with the outlying scale scores, presented with potential normality issues.

The item on the Victimization History Questionnaire Extended, which asked participants to rate the perceived physical severity of their sexual victimization experience(s), had acceptable kurtosis (1.67; i.e., within the range of \pm 1.96$)$, but was positively skewed (2.32). Within the PANAS-X, the Fear and Shyness scales were positively skewed (3.86 and 2.86, respectively), and slightly leptokurtic (5.61 and 3.12, respectively). The Guilt, Hostility, and Surprise scales, on the other hand, were positively skewed $(3.38,2.71,2.01$, respectively), but retained acceptable kurtosis $(1.67,1.86,-.68$, respectively). All IASR-B5 scales had skew and kurtosis values well under the cut-off of \pm 1.96 , thereby satisfying the assumption of normality.

All skewed and kurtotic variables were corrected using the appropriate transformation (see Tabachnick \& Fidell, 2013). Given that the item which asked about the physical severity of one's sexual victimization had minimal positive skew, it was 
corrected using a square root transformation. The skew became 1.70, and the kurtosis became .34 , thereby satisfying the assumption of normality. The Shyness, Hostility, and Surprise scales of the PANAS-X were each corrected using a square root transformation as well. The skew for Shyness became 1.14, and kurtosis became .79. The skew for Hostility became 1.55, and the kurtosis became .39. Lastly, the skew for Surprise became 1.61, and the kurtosis became -.93 . The Guilt and Fear scales were skewed to a greater extent, and could not be remedied using a square root transformation. Therefore, they were corrected using a logarithm transformation. For the Guilt scale, the skew became 1.66, and the kurtosis became -.18. Finally, the skew and kurtosis for the Fear scale both became .82. Analyses were conducted with both the transformed and uncorrected (i.e., original) items, and the change in the results was negligible. Therefore, for ease of interpretation, the uncorrected (i.e., original) scale items were used and are reported for all analyses in Study 1.

\section{Primary Analyses}

\section{Descriptive statistics.}

Victimization History Questionnaire Extended. The current thesis is unique in that it included measures related to the type of victimization the walkers experienced (i.e., violent and sexual), the time frame of their victimization (i.e., very recently [within the last few weeks], recently [within the last 6 months], quite a while ago [1-2 years ago], long ago [2-5 years ago], and very long ago [5 + years ago]), the frequency of their victimization (i.e., once, twice, 3-5 times, and $5+$ times), and the subjective severity (measured on multiple scales) of the event(s). Therefore, exploratory descriptive statistics 
for each of the measures related to sexual and violent victimization history are reported in Table 2 and 3 , respectively.

In terms of victimization history, approximately $35.5 \%(n=11)$ of the total sample (i.e., out of the total 31 walkers) reported that they had been sexually victimized. Of those who had been sexually victimized, $54.5 \%(n=6)$ had also been violently victimized, whereas $45.5 \%(n=5)$ reported that their victimization experience(s) had been exclusively sexual in nature. Thus, the majority of individuals who had histories of sexual victimization, also had histories of violent victimization. Contrary to previous studies (e.g., Murzynski \& Degelman, 1996; Ritchie, 2013), the current sample did not include significantly more female participants $(54.5, n=6, z=-.5)$ with a history of sexual victimization, relative to males $(45.5 \%, n=5, z=.8), \chi^{2}(1)=1.36, p=.244$; both genders were equally represented.

Participants who indicated that they had been victimized sexually were asked a series of questions related to the event. ${ }^{10}$ First, they were asked to indicate when the event occurred and then, how many times they had been a victim of a sexual crime. On average, most participants who had been sexually victimized $(45.5 \%, n=5)$ indicated that the event had occurred "very long ago (5+ years ago)," or "quite a while ago (1-2 years ago; $36.4 \%, n=4)$." In relation to the number of times individuals had experienced sexual victimization, the majority of participants reported that it had occurred "once" $(45.5 \%, n=5)$.

Next, participants were asked various questions related to the perceived severity of their victimization. For example, they were asked about the extent to which their

\footnotetext{
${ }^{10}$ Individuals who had been victimized multiple times were asked to refer to the most (subjectively) serious experience when completing the questionnaire.
} 
sexual victimization experience had caused them distress. Nearly all respondents $(81.8 \%$, $n=9$ ) indicated that the event had caused them "minimal distress (e.g., initial stress, which later dissipated; some symptoms present, etc.)," whereas a small proportion $(18.2 \%, n=2)$ reported that the experience had caused them "severe distress (e.g., experienced persistent, interfering symptoms; received counseling; notified authorities, etc.)."

Participants were also asked whether or not they felt afraid, helpless or horrified at the time when they were sexually victimized. Approximately $54.5 \%(n=6)$ responded that "yes" they had experienced such feelings, whereas $45.5 \%(n=5)$ had not experienced these same feelings. Relatedly, participants were asked whether or not they had been seriously injured, and if they believed that they were in danger or could be seriously injured, when they were sexually victimized. Nearly all of the respondents indicated that they had not been seriously injured $(81.8 \%, n=9)$; only $9.1 \%(n=1)$ had been injured as a result of being sexually victimized. Similarly, most participants $(72.7 \%$, $n=8$ ) did not believe that they were in danger or would be injured at the time when they were victimized; only three individuals $(27.3 \%)$ reported feelings of danger.

Next, participants were asked about the extent to which their sexual victimization bothered them emotionally, and for how long they were bothered. On average, the majority of participants were bothered "a little" $(45.5 \%, n=5)$ by the event. A number of participants were also bothered "somewhat" $(36.4 \%, n=4)$. Most participants were bothered for approximately "1 week" $(54.5 \%, n=6)$.

Lastly, participants were asked to rate, on a 5 -point scale $(1=$ not severe, $5=$ extremely severe), the perceived emotional, psychological, and physical severity, of their 
sexual victimization. In terms of emotional severity, $45.5 \%(n=5)$ rated their sexual victimization as a " 2 ." Most participants rated the psychological severity of their sexual victimization low (i.e., $27.3 \%$, [ $n=3$ ] rated the experience as a "1" [i.e., "not severe"], and $27.3 \%[n=3]$ rated it as a " 2 "). Lastly, over half of participants $(54.5 \%, n=6)$ indicated that the experience was not physically severe (i.e., they rated it as " 1 "). 
Table 2

Descriptive Statistics for Participants with a Sexual Victimization History

\begin{tabular}{|c|c|c|c|c|}
\hline Questions & $\%(n)$ & Mode & $M$ & $S D$ \\
\hline \multicolumn{5}{|l|}{ Experienced Sexual } \\
\hline \multicolumn{5}{|l|}{ Victimization: } \\
\hline Yes & $35.5(11)$ & & & \\
\hline No & $64.5(20)$ & & & \\
\hline \multicolumn{5}{|l|}{ Time Frame: ${ }^{\mathrm{a}}$} \\
\hline $5+$ years ago & $45.5(5)$ & \multirow{5}{*}{$5+$ years ago } & & \\
\hline $2-5$ years ago & $9.1(1)$ & & & \\
\hline $1-2$ years ago & $36.4(4)$ & & & \\
\hline Last 6 months & $0(0)$ & & & \\
\hline Last few weeks & $9.1(1)$ & & & \\
\hline \multicolumn{2}{|l|}{ Frequency: ${ }^{a}$} & \multirow[t]{5}{*}{ Once } & & \\
\hline Once & $45.5(5)$ & & & \\
\hline Twice & $18.2(2)$ & & & \\
\hline $3-5$ times & $27.3(3)$ & & & \\
\hline $5+$ times & $9.1 \%(1)$ & & & \\
\hline \multicolumn{5}{|l|}{ Severity } \\
\hline Distress: ${ }^{a}$ & & \multirow[t]{5}{*}{ Minimal } & & \\
\hline None & $0(0)$ & & & \\
\hline Minimal & $81.8(9)$ & & & \\
\hline Moderate & $0(0)$ & & & \\
\hline Severe & $18.2(2)$ & & & \\
\hline \multicolumn{5}{|l|}{ Feelings of Danger: ${ }^{a}$} \\
\hline Yes & $27.3(3)^{\mathrm{b}}$ & \multirow[t]{2}{*}{ No } & & \\
\hline No & $72.7(8)^{b}$ & & & \\
\hline \multicolumn{5}{|l|}{ Injury: ${ }^{a, b}$} \\
\hline Yes & $9.1(1)^{\mathrm{b}}$ & \multirow[t]{2}{*}{ No } & & \\
\hline No & $81.8(9)^{\mathrm{b}}$ & & & \\
\hline \multicolumn{5}{|l|}{ Feelings of Fear: ${ }^{a}$} \\
\hline Yes & $54.5(6)^{b}$ & \multirow[t]{2}{*}{ Yes } & & \\
\hline No & $45.5(5)^{b}$ & & & \\
\hline \multicolumn{5}{|c|}{ Length of Time Bothered: ${ }^{a, b}$} \\
\hline Not at all & $0(0)$ & \multirow{4}{*}{ One week } & & \\
\hline One week & $54.5(6)$ & & & \\
\hline 2-3 weeks & $9.1(1)$ & & & \\
\hline A month or more & $8.2(2)$ & & & \\
\hline \multicolumn{5}{|l|}{ Extent Bothered: a } \\
\hline Not at all & $0(0)$ & \multirow{5}{*}{ A little } & & \\
\hline A little & $45.5(5)$ & & & \\
\hline Somewhat & $36.4(4)$ & & & \\
\hline Much & $0(0)$ & & & \\
\hline Very much & $18.2(2)$ & & & \\
\hline
\end{tabular}




\begin{tabular}{|c|c|c|c|c|}
\hline Questions & $\%(n)$ & Mode & $M$ & $S D$ \\
\hline \multicolumn{5}{|c|}{ Rating of Emotional Severity: ${ }^{a}$} \\
\hline 1 & $18.2(2)$ & & \multirow{5}{*}{2.55} & \multirow{5}{*}{1.37} \\
\hline 2 & $45.5(5)$ & & & \\
\hline 3 & $18.2(2)$ & & & \\
\hline 4 & $0(0)$ & & & \\
\hline 5 & $18.2(2)$ & & & \\
\hline \multicolumn{5}{|c|}{ Rating of Psychological Severity: ${ }^{a}$} \\
\hline 1 & $27.3(3)$ & & \multirow{5}{*}{2.64} & \multirow{5}{*}{1.50} \\
\hline 2 & $27.3(3)$ & & & \\
\hline 3 & $18.2(2)$ & & & \\
\hline 4 & $9.1(1)$ & & & \\
\hline 5 & $18.2(2)$ & & & \\
\hline \multicolumn{5}{|c|}{ Rating of Physical Severity: a } \\
\hline 1 & $54.5(6)$ & & \multirow{5}{*}{1.91} & \multirow{5}{*}{1.30} \\
\hline 2 & $18.2(2)$ & & & \\
\hline 3 & $18.2(2)$ & & & \\
\hline 4 & $0(0)$ & & & \\
\hline 5 & $9.1(1)$ & & & \\
\hline
\end{tabular}

Note. Where the mean was not applicable (i.e., the items were categorical), the mode is provided. ${ }^{a}$ Percentages are based on the 11 participants that were sexually victimized. ${ }^{b}$ The remaining participant(s) provided a non-response by checking the box entitled "Check this box if you do not want to provide an answer to this question."

On the other hand, $41.9 \%(n=13)$ of the total sample indicated that they had been violently victimized. Of those who had been violently victimized, $46.2 \%(n=6)$ had also been sexually victimized; that is, $53.8 \%(n=7)$ reported that their victimization experience(s) were exclusively violent. In terms of gender, there was no significant difference between the number of males $(46.2 \%, n=6, z=.9)$ and the number of females $(53.8 \%, n=7, z=-.6)$ who reported a history of violent victimization, $\chi^{2}(1)=1.98, p=$ .160. In other words, a relatively equal number of males and females reported that they had been violently victimized.

Individuals who indicated that they had been a victim of a violent crime were asked to complete a series of questions pertaining to the event(s). First, they were asked to report when the event occurred. The majority of violently victimized participants $(46.2 \%, n=6)$ indicated that they had been victimized "very long ago ( $5+$ years ago)." A 
slightly lesser amount $(30.8 \%, n=4)$ indicated that they had been violently victimized "recently (within the last 6 months)." Next, participants were asked to report the number of times that they had been violently victimized. On average, most participants reported that they had been violently victimized " $5+$ times" $(38.5 \%, n=5$; i.e., the highest available option).

To capture the perceived severity of each participant's victimization experience, a series of questions asked about the participant's distress, feelings of helplessness, and injuries as a result of the event. Approximately $38.5 \%(n=5)$ indicated that their violent victimization experience had caused them "minimal distress (e.g., initial stress, which later dissipated; some symptoms present, etc.).” Another $38.5 \%(n=5)$ of participants reported that their violent victimization experience caused them "moderate distress (e.g., experienced certain long-lasting difficulties; notified authorities, etc.)."

Participants were asked to report whether or not they experienced feelings of fear, helplessness, or horror, when they were violently victimized. The majority of participants $(84.6 \%, n=11)$ indicated that they did experience such feelings, whereas $15.4 \%(n=2)$ did not report these same feelings. Participants were then asked to report whether or not they were seriously injured at the time of their victimization. Approximately $76.9 \%(n=$ 10) were not seriously injured, whereas $23.1 \%(n=3)$ reported that they did endure serious injuries. The number of participants who believed that they were in danger, or that they might be seriously injured when they were violently victimized $(46.2 \%, n=6)$, was relatively equal to the number who did not believe that they were in imminent danger $(53.8 \%, n=7)$. 
Participants were asked to report the extent to which their violent victimization experience bothered them. An equal number of participants indicated that the event bothered them "somewhat" $(30.8 \%, n=4)$, and "very much" $(30.8 \%, n=4)$. Relatedly, $38.5 \%(n=5)$ of individuals reported that their violent victimization experience bothered them, on average, 2-3 weeks.

The last three questions pertaining to violent victimization asked respondent's to rate (on a scale ranging from 1 [not severe] to 5 [extremely severe]) the degree to which they perceived their violent victimization to be emotionally severe, psychologically severe, and physically severe. The majority of individuals who had been a victim of a violent crime $(38.5 \%, n=5)$ reported that the emotional severity of their experience was average (i.e., they rated their victimization as a "3" on the 5-point scale). In terms of psychological severity, the majority of participants rated their violent victimization as " 4 " $(30.8 \%, n=4)$. Finally, most participants $(46.2 \%, n=6)$ rated the physical severity of their violent victimization as "not severe" (i.e., "1" on the 5-point scale). 
Table 3

Descriptive Statistics for Participants with a Violent Victimization History

\begin{tabular}{|c|c|c|c|c|}
\hline Questions & $\%(n)$ & Mode & $M$ & $S D$ \\
\hline \multicolumn{5}{|l|}{ Experienced Sexual } \\
\hline \multicolumn{5}{|l|}{ Victimization: } \\
\hline Yes & $41.9(13)$ & & & \\
\hline No & $58.1(18)$ & & & \\
\hline \multicolumn{5}{|l|}{ Time Frame: ${ }^{\mathrm{a}}$} \\
\hline $5+$ years ago & $46.2(6)$ & \multirow{5}{*}{$5+$ years ago } & & \\
\hline $2-5$ years ago & $7.7(1)$ & & & \\
\hline $1-2$ years ago & $15.4(2)$ & & & \\
\hline Last 6 months & $30.8(4)$ & & & \\
\hline Last few weeks & $0(0)$ & & & \\
\hline \multicolumn{2}{|l|}{ Frequency: a, b } & \multirow[t]{5}{*}{$5+$ times } & & \\
\hline Once & $23.1(3)$ & & & \\
\hline Twice & $15.4(2)$ & & & \\
\hline 3-5 times & $15.4(2)$ & & & \\
\hline $5+$ times & $38.5 \%(5)$ & & & \\
\hline \multicolumn{5}{|l|}{ Severity } \\
\hline Distress: ${ }^{\mathrm{a}, \mathrm{b}}$ & & \multirow[t]{5}{*}{ Minimal $^{\mathrm{c}}$} & & \\
\hline None & $0(0)$ & & & \\
\hline Minimal & $38.5(5)$ & & & \\
\hline Moderate & $38.5(5)$ & & & \\
\hline Severe & $15.4(2)$ & & & \\
\hline \multicolumn{5}{|c|}{ Feelings of Danger: ${ }^{a}$} \\
\hline Yes & $46.2(6)^{\mathrm{b}}$ & \multirow[t]{2}{*}{ No } & & \\
\hline No & $53.8(7)^{b}$ & & & \\
\hline \multicolumn{5}{|l|}{ Injury: ${ }^{a}$} \\
\hline Yes & $23.1(3)^{\mathrm{b}}$ & \multirow[t]{2}{*}{ No } & & \\
\hline No & $76.9(10)^{\mathrm{b}}$ & & & \\
\hline \multicolumn{5}{|l|}{ Feelings of Fear: ${ }^{a}$} \\
\hline Yes & $84.6(11)^{\mathrm{b}}$ & \multirow[t]{2}{*}{ Yes } & & \\
\hline No & $15.4(2)^{\mathrm{b}}$ & & & \\
\hline \multicolumn{5}{|c|}{ Length of Time Bothered: ${ }^{a, b}$} \\
\hline Not at all & $23.1(3)$ & \multirow{4}{*}{ 2-3 weeks } & & \\
\hline One week & $15.4(2)$ & & & \\
\hline 2-3 weeks & $38.5(5)$ & & & \\
\hline A month or more & $15.4(2)$ & & & \\
\hline \multicolumn{5}{|l|}{ Extent Bothered: a } \\
\hline Not at all & $0(0)$ & \multirow{5}{*}{ Somewhat ${ }^{\mathrm{c}}$} & & \\
\hline A little & $15.4(2)$ & & & \\
\hline Somewhat & $30.8(4)$ & & & \\
\hline Much & $23.1(3)$ & & & \\
\hline Very much & $30.8(4)$ & & & \\
\hline
\end{tabular}




\begin{tabular}{|c|c|c|c|c|}
\hline Questions & $\%(n)$ & Mode & $M$ & $S D$ \\
\hline \multicolumn{5}{|c|}{ Rating of Emotional Severity: ${ }^{\text {a }}$} \\
\hline 1 & $15.4(2)$ & & \multirow{5}{*}{2.85} & \multirow{5}{*}{1.07} \\
\hline 2 & $15.4(2)$ & & & \\
\hline 3 & $38.5(5)$ & & & \\
\hline 4 & $30.8(4)$ & & & \\
\hline 5 & $0(0)$ & & & \\
\hline \multicolumn{5}{|c|}{ Rating of Psychological Severity: ${ }^{a, b}$} \\
\hline 1 & $23.1(3)$ & & \multirow{5}{*}{2.62} & \multirow{5}{*}{1.19} \\
\hline 2 & $0(0)$ & & & \\
\hline 3 & $23.1(3)$ & & & \\
\hline 4 & $30.8(4)$ & & & \\
\hline 5 & $0(0)$ & & & \\
\hline \multicolumn{5}{|c|}{ Rating of Physical Severity: a } \\
\hline 1 & $46.2(6)$ & & \multirow{5}{*}{2.08} & \multirow{5}{*}{1.19} \\
\hline 2 & $15.4(2)$ & & & \\
\hline 3 & $23.1(3)$ & & & \\
\hline 4 & $15.4(2)$ & & & \\
\hline 5 & $0(0)$ & & & \\
\hline
\end{tabular}

Note. Where the mean was not applicable (i.e., the items were categorical), the mode is provided.

${ }^{a}$ Percentages are based on the 13 participants that were violently victimized. ${ }^{b}$ The remaining participant(s) provided a non-response by checking the box entitled "Check this box if you do not want to provide an answer to this question."

${ }^{\mathrm{c}}$ Multiple modes were present; the lowest mode is provided.

Revised Interpersonal Adjective Scales-Big Five. To obtain the total score for each of the scales in the IASR-B5, the ratings for the adjectives contained in each scale were summed. The summed scores were then divided by the total number of adjectives present within that scale (i.e., the mean was calculated for each scale; Trapnell \& Wiggins, 1990; Wiggins et al., 1988). Descriptive statistics for each scale are reported in Table 4.

The majority of individuals scored highest on the warm-agreeable scale $(M=$ $6.06, S D=1.08)$ and lowest on the cold-hearted scale $(M=2.73, S D=1.01)$. The "undergraduate norm" reported in Wiggins et al. (1988) is similar; students tend to score highest on the warm-agreeable scale $(M=5.96, S D=.81)$, and lowest on the cold-hearted 
scale $(M=2.54, S D=.85) .{ }^{11}$ In other words, on average, students tend to report that the warm-agreeable traits are "quite accurate" of themselves, whereas the traits indicative of cold-heartedness, are a "quite inaccurate" representation of themselves.

In regards to the main scales of interest, participants tended to report that the unassured-submissive traits were a "slightly inaccurate" $(M=4.00, S D=.99)$ representation of how they viewed their personality. Interestingly, however, individuals tended to report that the characteristics associated with the assured-dominant scale were a "slightly accurate" portrayal of themselves $(M=5.00, S D=.95)$. The present findings were very similar to the undergraduate norm; on average, students report that the unassured-submissive traits are "slightly inaccurate" $(M=4.00, S D=1.06)$ of themselves, while the assured-dominant traits are "slightly accurate" $(M=4.98, S D=$ .97) of themselves.

The three scales which measure the Big Five personality features were also examined. On average, individuals reported that the traits which encompassed openness to experience and conscientiousness were a "quite accurate" portrayal of themselves $(M=$ $6.00, S D=.86 ; M=5.77, S D=1.09$, respectively). The characteristics associated with neuroticism, on the other hand, were generally considered to be "slightly inaccurate" by participants $(M=4.47, S D=1.23)$. The current findings were fairly similar to the undergraduate norms presented by Wiggins et al. (1988): On average, undergraduate students tend to report that the characteristics that comprise the openness to experience scale are "slightly accurate" $(M=5.48, S D=.75)$, whereas the norm for

\footnotetext{
${ }^{11}$ The "undergraduate norm" for each octant in the IAS-R is based on a sample of 688 female and 473 male undergraduate students (Wiggins et al., 1988).
} 
conscientiousness is "quite accurate" $(M=5.67, S D=.95)$, and the norm for neuroticism is "slightly accurate" $(M=4.64, S D=.89)$.

In terms of gender, males and females differed significantly from one another on two scales: Female participants $(M=6.37, S D=.93)$ scored higher than male participants $(M=5.40, S D=1.12)$ on the warm-agreeable scale, $t(29)=2.54, p=.017$. Females $(M=$ $4.95, S D=1.18)$ also scored higher than males $(M=3.68, S D=.95)$ on the unassumingingenuous scale, $t(29)=2.96, p=.006$. There were no significant differences between the sexes on any of the other scales in the IASR-B5. Independent samples t-test statistics for the remaining scales are presented in Table 4. 
Table 4

Descriptive Statistics for the IASR-B5 Scales

\begin{tabular}{|c|c|c|c|c|c|c|c|c|}
\hline \multirow{2}{*}{ IASR-B5 Scales ${ }^{\mathrm{a}}$} & \multicolumn{2}{|c|}{ Total Sample } & \multicolumn{2}{|c|}{ Males $^{\mathrm{d}}$} & \multicolumn{2}{|c|}{ Females $^{\mathrm{e}}$} & \multirow[b]{2}{*}{$t$} & \multirow[b]{2}{*}{$p$} \\
\hline & $M$ & $S D$ & $M$ & $S D$ & $M$ & $S D$ & & \\
\hline Conscientiousness $^{\mathrm{b}}$ & 5.77 & 1.09 & 5.86 & .85 & 5.73 & 1.20 & -.29 & .776 \\
\hline Neuroticism $^{\mathrm{b}}$ & 4.47 & 1.23 & 4.32 & 1.27 & 4.54 & 1.24 & .46 & .646 \\
\hline Openness to Experience ${ }^{b}$ & 6.00 & .86 & 6.40 & .91 & 5.82 & .79 & -1.84 & .076 \\
\hline Assured-Dominant & 5.00 & .95 & 4.91 & .66 & 5.04 & 1.07 & .33 & .742 \\
\hline Gregarious-Extraverted & 5.66 & 1.25 & 5.11 & 1.12 & 5.92 & 1.24 & 1.75 & .091 \\
\hline Warm-Agreeable & 6.06 & 1.08 & 5.40 & 1.12 & 6.37 & .93 & 2.54 & $.017 *$ \\
\hline Unassuming-Ingenuous & 4.54 & 1.25 & 3.68 & .95 & 4.95 & 1.18 & 2.96 & $.006^{*}$ \\
\hline Aloof-Introverted & 3.30 & 1.27 & 3.81 & 1.01 & 3.05 & 1.33 & -1.60 & .122 \\
\hline Cold-Hearted & 2.73 & 1.01 & 3.20 & 1.00 & 2.51 & .96 & -1.84 & .077 \\
\hline Arrogant-Calculating & 3.53 & 1.39 & 4.14 & 1.66 & 3.24 & 1.18 & -1.73 & .095 \\
\hline Unassured-Submissive & 4.00 & .99 & 3.84 & .84 & 4.01 & 1.07 & .45 & .653 \\
\hline
\end{tabular}

Note. IASR-B5 $=$ Revised Interpersonal Adjective Scales Big Five. $* p<.05$. The scale ranged from 1 (extremely inaccurate) to 8 (extremely accurate). Higher scores are indicative of greater participant agreement on the items in that scale, whereas lower scores are indicative of less agreement. Levene's Test was not significant at $p<.05$ for any of the scales. Therefore, equal variances were assumed for all t-tests (Field, 2009).

${ }^{a}$ Each scale in the IASR-B5 was scored by calculating the mean from the responses made to the individual adjectives that comprised each scale (i.e., participant responses on the items "timid," "unaggressive," "unbold," "shy," "meek," "unauthoritative," "forceless," "bashful" were added and divided by eight in order to obtain the total scale score for "Unassured-Submissive;" Trapnell \& Wiggins, 1990). ' There were twenty adjectives that comprised each Big Five scale (i.e., "Conscientiousness," "Neuroticism," and "Openness to Experience"). Several items in the Big Five scales were reverse coded according to Trapnell and Wiggins (1990); all items were then summed and divided by twenty to obtain the total scale score. ${ }^{\mathrm{c}} N=31 .{ }^{\mathrm{d}} n=10 .{ }^{\mathrm{e}} n=21$. 
Manual for the Positive and Negative Affect Schedule Expanded Form. The PANAS-X contains thirteen scales; each scale is comprised of various attributes (e.g., cheerful). The number of items that comprise each scale varies. For example, the General Positive Affect scale contains the items: Active, alert, attentive, enthusiastic, excited, inspired, interested, proud, strong, and determined. The higher order Serenity scale, on the other hand, contains the items: Calm, relaxed, and at ease. To obtain the total score for a particular scale, the ratings for each of the items contained in the scale were summed (Watson \& Clark, 1994). Descriptive statistics for each scale are reported in Table 5.

On average, participants scored highest on the General Positive Affect $(M=$ 31.29, $S D=7.35)$ and Joviality $(M=25.55, S D=6.83)$ scales. Alternatively, participants rated themselves lowest on the Surprise $(M=5.55, S D=2.50)$ and Shyness $(M=7.90$, $S D=3.19$ ) scales. The current findings were comparable with those observed during the development and testing of the PANAS-X. ${ }^{12}$ Watson and Clark (1994) reported high scores for the General Positive Affect $(M=32.60, S D=7.10)$ and Joviality $(M=26.80$, $S D=6.60)$ scales; lower scores were observed by Watson and Clark (1994) on the Surprise $(M=6.80, S D=2.80)$ and Shyness $(M=7.70, S D=3.10)$ scales.

The General Negative Affect, Fear, Sadness, Guilt, and Hostility scales were of particular interest for the current study. Participants scored highest on the General Negative Affect $(M=20.68, S D=5.92)$ scale. The Fear $(M=11.39, S D=3.76)$, Sadness $(M=11.39, S D=4.40)$, Guilt $(M=11.59, S D=5.40)$, and Hostility $(M=11.06, S D=$

\footnotetext{
${ }^{12}$ The norms observed in the development and testing of the two basic scales in the PANAS-X (i.e., General Positive Affect and General Negative Affect) are based on a sample of 2,076 undergraduate students who were given the same temporal instructions as the current sample (i.e., "Indicate to what extent you have felt this way during the past few weeks;" Watson \& Clark, 1994).
} 
4.04) scales had very similar scale scores. Again, the scale scores were consistent with those observed in development and testing (Watson \& Clark, 1994). The scale score reported by Watson and Clark (1994) for General Negative Affect was $20.20(S D=7.20)$. The scores for Fear $(M=12.30, S D=4.90)$, Sadness $(M=11.70, S D=4.80)$, Guilt $(M=$ 12.00, $S D=5.20)$, and Hostility $(M=12.90, S D=5.00)$ that were observed in development and testing, were slightly higher than those found in the present sample. ${ }^{13}$ In terms of gender, males and females differed significantly from one another on the Attentiveness scale; interestingly, male participants $(M=14.70, S D=2.50)$ scored higher than female participants $(M=12.33, S D=1.93), t(29)=-2.90, p=.007$. The Attentiveness scale contains the items "alert," "attentive," "concentrating," and "determined." There were no significant differences between the sexes on any of the other scales in the PANAS-X. Independent samples t-test statistics for the remaining scales are presented in Table 5 .

\footnotetext{
${ }^{13}$ The norms observed in development and testing for each of the higher order scales in the PANAS-X, are based on a sample of 678 undergraduate students who were given the same temporal instructions as the current sample (i.e., "Indicate to what extent you have felt this way during the past few weeks;" Watson \& Clark, 1994).
} 
Table 5

Descriptive Statistics for the PANAS-X Scales

\begin{tabular}{|c|c|c|c|c|c|c|c|c|}
\hline \multirow{2}{*}{ PANAS-X Scales $^{a}$} & \multicolumn{2}{|c|}{ Total Sampleb } & \multicolumn{2}{|c|}{ Males $^{\mathrm{c}}$} & \multicolumn{2}{|c|}{ Females $^{\mathrm{d}}$} & \multirow[b]{2}{*}{$t$} & \multirow[b]{2}{*}{$p$} \\
\hline & $M$ & $S D$ & $M$ & $S D$ & $M$ & $S D$ & & \\
\hline General Negative Affect & 20.68 & 5.92 & 20.30 & 7.83 & 20.86 & 4.99 & .24 & .811 \\
\hline Fear & 11.39 & 3.76 & 12.30 & 5.68 & 10.95 & 2.46 & -.72 & .488 \\
\hline Sadness & 11.39 & 4.40 & 10.70 & 4.85 & 11.71 & 4.26 & .59 & .558 \\
\hline Guilt & 11.59 & 5.40 & 12.20 & 6.30 & 11.29 & 5.06 & -.43 & .667 \\
\hline Hostility & 11.06 & 4.04 & 10.60 & 3.69 & 11.29 & 4.27 & .44 & .666 \\
\hline Shyness & 7.90 & 3.19 & 8.50 & 3.27 & 7.62 & 3.19 & -.71 & .481 \\
\hline Fatigue & 13.26 & 3.76 & 13.00 & 3.33 & 13.38 & 4.02 & .26 & .797 \\
\hline General Positive Affect & 31.29 & 7.35 & 32.90 & 8.48 & 30.52 & 6.85 & -.84 & .410 \\
\hline Joviality & 25.55 & 6.83 & 24.40 & 7.24 & 26.10 & 6.73 & .64 & .527 \\
\hline Self-Assurance & 16.06 & 5.27 & 17.20 & 5.39 & 15.52 & 5.26 & -.82 & .417 \\
\hline Attentiveness & 13.10 & 2.37 & 14.70 & 2.50 & 12.33 & 1.93 & -2.90 & $.007 *$ \\
\hline Serenity & 9.32 & 2.73 & 9.40 & 2.84 & 9.29 & 2.74 & -.11 & .915 \\
\hline Surprise & 5.55 & 2.50 & 6.00 & 2.49 & 5.33 & 2.54 & -.69 & .497 \\
\hline
\end{tabular}

Note. PANAS-X = Manual for the Positive and Negative Affect Schedule Expanded Form. * $p<.01$. The scale ranged from 1 (very slightly or not at all) to 5 (extremely). Higher scores are indicative of greater participant agreement on the items in that scale, whereas lower scores are indicative of less agreement. Levene's Test indicated unequal variances for the "Fear" scale $(F=4.72, p=.038)$; degrees of freedom were thus adjusted from 29 to 10.64 . Levene's Test was not significant at $p<.05$ for any of the other scales. Therefore, equal variances were assumed for the remaining t-tests (Field, 2009).

a Each scale in the PANAS-X was scored by summing the responses to the items comprising that scale (i.e., participant responses on the items "surprised,"

"amazed" and "astonished" were summed in order to obtain the total scale score for "Surprise;" Watson $\&$ Clark, 1994$) .{ }^{\mathrm{b}} N=31 .{ }^{\mathrm{c}} n=10 .{ }^{\mathrm{d}} n=21$. 
Gait analysis. All 31 students participated in the movement portion of Study 1. However, because there were issues with reflection and calibration, only 28 videos were useable. Of the 28 videos, $64.3 \%(n=18)$ were female walkers, and $35.7 \%(n=10)$ were male walkers. Approximately $57.1 \%(n=16)$ of the walkers reported that they had a history of victimization. There was an equal ratio of individuals who reported a history of violent victimization $(39.3 \%, n=11)$ and sexual victimization $(39.3 \%, n=11)$. The remaining $42.9 \%(n=12)$ of individuals had not been victimized.

The walkers' movements were coded by two independent raters; both raters coded all 28 walkers. Both raters were blind to the victimization history of the walkers. The movement code used (see Appendix A) was adopted from Ritchie (2014), and based on the significant findings in the original research by Grayson and Stein (1981). Recall that the movement code included five categories: Stride length (e.g., medium), type of weight shift (e.g., lateral), type of walk (e.g., postural), body movement (e.g., unilateral), and feet (e.g., swung). Given the difficulty in coding stride length and foot movement, two (10-second) videos were created for every walker. One video captured the participant walking from the back (i.e., as if the individual was walking away from the camera), and the other video captured them walking from the side. Both camera angles were utilized when coding the walkers' movements. However, consistent with Book et al. (2013), Wheeler et al. (2009), and Ritchie (2014), only the back view was shown to the observers in Study 2.

Inter-judge agreement was reasonably high for all of the movement categories (Cohen's kappa ranged from .75 to 1.00 ; weight shift $=.76$, type of walk $=.75$, stride length $=.76$, feet $=.77$, body movement $=1.00$; agreement for all categories was 
significant at $p<.001)$. Again, in line with previous studies, the ratings were tallied across categories, and each walker was provided a total vulnerability score. For example, if a walker was rated as having a medium stride length, a three-dimensional weight shift, a gestural walk, unilateral body movement, and lifted feet, their vulnerability score would be three; a gestural walk, unilateral movement, and lifted foot movements are considered "vulnerable" movements according to Grayson and Stein (1981), and would thus be tallied together. The remaining characteristics (i.e., medium stride length, and threedimensional weight shift) are considered non-vulnerable movements and would not add to the walkers' total vulnerability score. Low vulnerability scores (e.g., 0, 1, 2) indicated that the walker had little or no vulnerability cues present in their gait pattern. Higher scores, up to a possible total score of five, indicated that the walker had most, or all of the vulnerability features present in their gait pattern.

Approximately $53.5 \%(n=15)$ of the walkers had a low total vulnerability score (i.e., they scored between 0 and 2). On the other hand, $46.4 \%(n=13)$ of the walkers had a high total vulnerability score (i.e., they scored between 3 and 5). On average, most walkers scored in the lower range $(M=2.39, S D=1.57)$. Therefore, the majority of individuals were perceived by the coders as having a gait style with low to average vulnerability. There was no significant difference in vulnerability between male $(M=$ $2.40, S D=1.65)$ and female walkers $(M=2.39, S D=1.58), t(26)=-.018, p=.986$. 
Analyses. ${ }^{14}$ The areas of research pertaining to "Study 1: The "Walkers"” are reiterated in Table 6 . The analyses used to examine each area are noted and described in more detail following the table.

Table 6

Study 1 Analyses

Areas of Research

Analysis

1) Gait pattern vulnerability

Correlations

Mann-Whitney U

2) The relationship between victimization

Correlations

history, gait, and personality

Mediated Regression

3) The relationship between victimization

Correlations $^{15}$

history, gait, and affect

4) The effect of time, frequency, severity,

Descriptive Statistics

and type of victimization, on gait

Correlations

Gait pattern vulnerability. First, a Levene's Test was conducted to ensure that the distribution of scores for individuals with a victimization history was similar to the distribution of scores for the control group (i.e., individuals without a victimization history); the test was not significant at $p<.05(F=2.19, p=.151)$. It could thus be assumed that the two groups had a similar distribution of scores on the gait vulnerability scale variable.

\footnotetext{
${ }^{14}$ One-tailed tests were used for all analyses (in both Study 1 and Study 2) in which a directional hypothesis had been made. Two-tailed tests were used when the research question outlined was exploratory.

${ }^{15}$ Given the lack of association between gait, victimization history, and the affect scale variables (reported in greater detail below), it was not feasible to conduct a mediated regression similar to that which was performed for personality.
} 
A Rank-Biserial Correlation was conducted to examine the relationship between each individual's gait pattern (an ordinal scale variable ranging from 0 [no vulnerability features present) to 5 [all possible vulnerability features present]) and their victimization history (a nominal dichotomous variable; 1 = they reported either sexual and/or violent victimization; the control group was coded as 0). As expected, gait pattern and victimization history were significantly correlated, $\left(r_{\mathrm{rb}}=.55, p=.003\right)$. Therefore, individuals who reported that they had experienced either sexual and/or violent victimization were significantly more likely to have a vulnerable coded gait pattern.

A Mann-Whitney U test was also conducted and similar findings were observed. Median latencies in the victimized group and non-victimized group were 3.00 and 1.00, respectively, and the groups differed significantly $\left(U=36.00, Z=-2.86, p=.004,{ }^{16} r=\right.$ .54). As expected, the vulnerability score was significantly greater for individuals with a victimization history (sexual and/or violent; Mean rank $=18.25$ ) than for individuals without a victimization history (Mean rank $=9.50)$.

The relationship between victimization history, gait, and personality. The personality of the sample of walkers was examined. Only one participant who scored high $^{17}$ on submissive traits and negative affect was removed from this portion of the analyses so that affect did not confound the results. Victimization history $(1=$ they reported sexual and/or violent victimization; the control group was coded as 0) was correlated (using Point-Biserial Correlations) with total scale scores for each personality

\footnotetext{
${ }^{16}$ Given the small sample size (i.e., $<60$ ), exact $p$-values are provided for all Mann-Whitney U statistics in Study 1 .

17 "High" on the unassured-submissive (HI) scale in the IASR-B5 was defined as one standard deviation (1.06) above the average undergraduate "norm" for males and females (4.00) reported by Wiggins et al. (1988). Similarly, "high" on the negative affect scale in the PANAS-X was defined as one standard deviation (7.2) above the average undergraduate "norm" (20.2) reported by Watson and Clark (1994).
} 
trait measured in the IASR-B5 (interval variables; assured-dominant [PA], gregariousextraverted [NO], warm-agreeable [LM], unassuming-ingenuous [JK], unassuredsubmissive $[\mathrm{HI}]$, aloof-introverted [FG], cold-hearted [DE], arrogant-calculating [BC], conscientiousness [CONSC], neuroticism [NEUR], and openness to experience [OPNS]). Contrary to expectation, little to no relationship was observed between the Unassured-Submissive scale in the IASR-B5 and self-reported victimization history $\left(r_{\mathrm{pb}}\right.$ $=.03, p=.881 ;$ see Table 7). Interestingly however, although not significant, the relationship between the Assured-Dominant scale and victimization history was positive $\left(r_{\mathrm{pb}}=.16, p=.390\right)$. Therefore, it appears that victimization is more likely to be associated with dominance than with submissiveness. The highest correlations observed were between the Gregarious-Extraverted scale and victimization history $\left(r_{\mathrm{pb}}=-.27, p=\right.$ .149), and the Openness to Experience scale and victimization history $\left(r_{\mathrm{pb}}=.21, p=\right.$ $.270)$. 
Table 7

Correlations Between Personality and Victimization History

\begin{tabular}{|c|c|c|}
\hline \multirow[b]{2}{*}{ IASR-B5 Scales } & \multicolumn{2}{|c|}{ Victimization History } \\
\hline & $r_{p b}$ & $P$ \\
\hline Unassured-Submissive & .03 & .881 \\
\hline Assured-Dominant & .16 & .390 \\
\hline Gregarious-Extraverted & -.27 & .149 \\
\hline Warm-Agreeable & -.02 & .933 \\
\hline Unassuming-Ingenuous & -.03 & .857 \\
\hline Aloof-Introverted & .09 & .648 \\
\hline Cold-Hearted & .10 & .615 \\
\hline Arrogant-Calculating & .02 & .933 \\
\hline Conscientiousness & -.09 & .657 \\
\hline Neuroticism & -.09 & .622 \\
\hline Openness to Experience & .21 & .270 \\
\hline
\end{tabular}


Next, bivariate correlations were calculated between gait and the total scale scores for each personality trait measured in the IASR-B5. Given that gait pattern was an ordinal variable (i.e., $0=$ no vulnerable cues present in gait; $5=$ all vulnerable cues present in gait), and the IASR-B5 scales could be considered interval variables, Spearman's RankOrder Correlation was used. Recall that a strong, positive correlation was expected between vulnerable-coded gait patterns and the Unassured-Submissive scale; weaker correlations were expected between vulnerable-coded gait patterns and the AssuredDominant scale.

Contrary to expectation, a significant positive correlation was observed between gait and the Assured-Dominant scale $\left(r_{\mathrm{s}}[25]=.46, p=.016\right) .{ }^{18}$ The correlation between gait and the Unassured-Submissive scale, on the other hand, was negative, and did not reach statistical significance $\left(r_{\mathrm{s}}[25]=-.34, p=.083\right)$. The findings suggest that individuals with more vulnerable features present in their gait are significantly more likely to self-report dominant personality characteristics. In other words, those with higher levels of vulnerability cues are actually less likely to have a submissive personality. Table 8 outlines the various correlations between the IASR-B5 scales and gait.

\footnotetext{
${ }^{18}$ Spearman's Correlation assumes that there is a monotonic relationship between each of the two variables of interest. Therefore, throughout Study 1 and 2, scatterplots were conducted to ensure that the assumption was met; no violations were observed.
} 
Table 8

Correlations Between Personality and Gait

\begin{tabular}{lcc}
\hline & \multicolumn{3}{c}{ Gait Pattern } \\
\cline { 2 - 3 } IASR-B5 Scale Variables & $r_{\mathrm{s}}$ & $p$ \\
\hline Unassured-Submissive & -.34 & .083 \\
Assured-Dominant & .46 & $.016^{*}$ \\
Gregarious-Extraverted & .05 & .813 \\
Warm-Agreeable & .30 & .126 \\
Unassuming-Ingenuous & .07 & .739 \\
Aloof-Introverted & -.10 & .609 \\
Cold-Hearted & -.28 & .152 \\
Arrogant-Calculating & -.21 & .290 \\
Conscientiousness & -.01 & .945 \\
Neuroticism & -.13 & .524 \\
Openness to Experience & .26 & .197 \\
\hline
\end{tabular}

Note. $N=27$; three videos were not useable and a gait vulnerability score could not be computed for them. They were removed from the present analyses. One participant scored highly on both the UnassuredSubmissive scale and on the General Negative Affect scale. They were also removed from the analyses so that affect did not confound the results. IASR-B5 = Revised Interpersonal Adjective Scales Big Five. $r_{\mathrm{s}}=$ Spearman's Rank-Order Correlation. ${ }^{*} p<.05$. Higher scores on the IASR-B5 are indicative of greater participant agreement on the items in that scale, whereas lower scores are indicative of less agreement. The gait pattern scale variable ranged from $0=$ no vulnerable cues present, to $5=$ all vulnerable cues present.

Lastly, a mediated ordinal regression ${ }^{19}$ was conducted to examine whether personality (dominance in this case because it was the one variable that correlated significantly with gait) could account for the relationship between victimization history and the vulnerable gait pattern observed in the participants. The expectation was that personality would act as a partial mediator between victimization history and gait.

The first step involved in classic mediation testing is to regress the predictor (victimization history) on the outcome (gait; path c in Figure 2). If a total effect exists, one can move on to the second and third steps. The second step involves regressing the predictor on the mediator (i.e., regressing victimization history on Assured-Dominance; path a). In the third step, the mediator is regressed on the outcome (i.e., regressing

\footnotetext{
${ }^{19}$ Although a number of tests were conducted on the same data, a Bonferroni adjustment was not performed to control for familywise error rate. Given Study 1's small sample size, the decision not to apply a correction is consistent with current discussions in the literature (Bender \& Lange, 2001).
} 
Assured-Dominance on gait; path b), while the predictor is controlled for (i.e., victimization history). In the final step, one can determine whether or not the mediator either completely or partially explains the relationship between the independent variable and the outcome. To establish that Assured-Dominance completely mediates the relationship between victimization history and gait, the effect of victimization history on gait (when Assured-Dominance is controlled for; path c') would have become zero. Partial mediation is typically established when the direct effect is significant (i.e., path c'), but becomes reduced in size when the mediator is introduced.

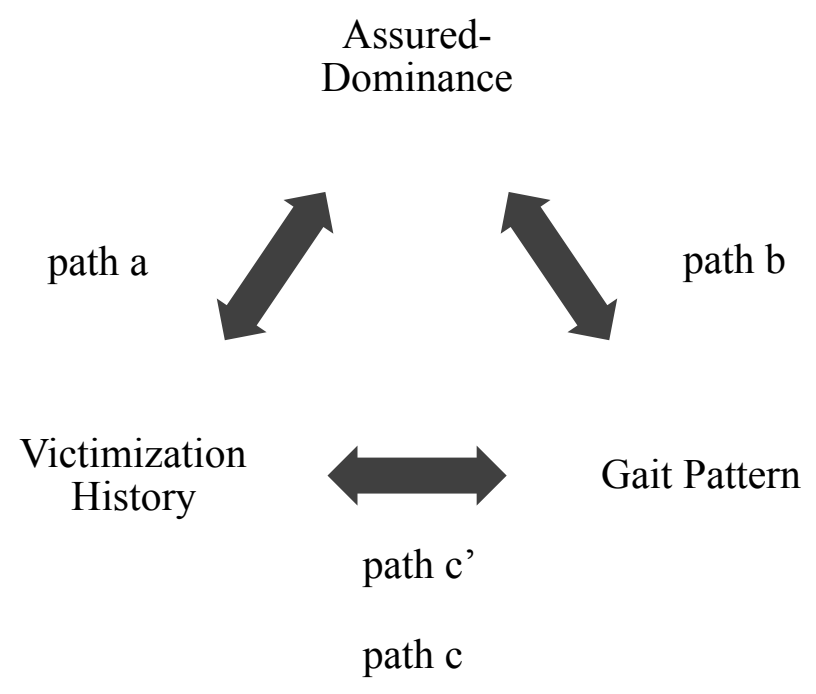

Figure 2. Mediation model examining the relationship between victimization history, gait pattern, and personality

Some researchers have argued that certain steps and/or rules governing classic mediation analysis are unnecessary, and/or that significance testing should not be used to establish whether or not mediation is present (e.g., James \& Brett, 1984). Indeed, it has been suggested that simply obtaining the product of path a and path $b$ provides an estimate of the "indirect effect" (i.e., it is equivalent to subtracting path c from path c;' 
this difference between the total and direct effect is needed to determine that mediation has occurred).

Hayes' (2013) "Process" macro (which is an add-on program installed into an existing SPSS software package) attempts to account for differences in mediation testing by calculating confidence intervals; these confidence intervals are bootstrapped and provide an estimate of the indirect effect. If the confidence interval for the indirect effect does not contain zero, one can assume that mediation is present. The Sobel Test is also included in the output and allows one to confirm (through significance testing) whether or not genuine mediation has occurred. "Process" was used to conduct the present mediation analysis.

Victimization history was entered as the predictor, the walker's self-reported score for the Assured-Dominance scale was entered as the mediator, and gait pattern was entered as the outcome variable. The total effect was statistically significant (path c; b = $1.71, p=.002,95 \% \mathrm{CI}[.67,2.75])$. Therefore, victimization history significantly predicted the vulnerability level of one's gait pattern. More specifically, on average, those who self-reported that they had been violently and/or sexually victimized received higher gait vulnerability scores. Indeed, victimization history explained $30 \%$ of the variance in gait pattern scores.

A significant relationship was also observed between personality (i.e., AssuredDominance) and gait pattern (when victimization history was controlled for; path $b ; b=$ $.55, p=.043,95 \%$ CI $[.02,1.09]) ;$ the regression coefficient indicated that as dominance ratings increased, so did the vulnerability score of one's gait pattern. There was also a 
significant relationship observed between victimization history and gait pattern (when Assured-Dominance was controlled for; path c;' $\mathrm{b}=1.41, p=.012,95 \% \mathrm{CI}[.34,2.48]$ ).

However, victimization history did not significantly predict dominance (i.e., path $\mathrm{a} ; \mathrm{b}=.54, p=.104,95 \% \mathrm{CI}[-.12,1.20])$, and previous victimization only accounted for $10.4 \%$ of the variance in Assured-Dominance scores. Interestingly however, given the positive regression coefficient, it appeared that individuals with sexual and/or violent victimization histories had more dominant personalities relative to those who had not been victimized. It is possible that a more comprehensive measure of dominance would confirm the replicability of the finding.

The $95 \%$ confidence interval for the indirect effect $\left(a^{*} b\right)$ included zero (and $b=0$ refers to no effect). Therefore, mediation could not be confirmed ( $a b=.30,95 \%$ CI [-.04, .96]); dominance did not mediate the relationship between victimization history and gait pattern. Indeed, the mediator only accounted for $17 \%$ (percent mediation $\left[P_{M}\right]=.17$ ) of the total effect. The completely standardized indirect effect $\left(a b_{c s}\right)$ was approximately $10 \%$. The Sobel Test was insignificant $(\mathrm{b}=.30, z=1.24, p=.215)$ and confirmed that genuine mediation had not occurred. Thus, it appears that while individuals with victimization histories tend to have a more vulnerable gait pattern, this elevated tendency cannot be attributed to their personality features (at least, as these personality features have been measured in the current sample of participants).

As some researchers may prefer classic mediation testing over the use of "Process," and/or wish to interpret the log odds of each regression equation, the manual method was also conducted and is reported in Appendix K. As noted, confidence 
intervals are bootstrapped in "Process" (Hayes, 2013); therefore, the regression coefficients differed between methods, but the pattern of results remained the same.

The relationship between victimization history, gait, and affect. The affect of the sample of walkers was examined. Again, the participant who scored highly on submissive traits and negative affect was removed from this portion of the analyses so that personality did not confound the results. Victimization history $(1=$ they reported either sexual and/or violent victimization; the control group was coded as 0) was correlated (using Point-Biserial Correlations) with total scores for each scale measured in the PANAS-X (interval variables; General Negative Affect, Fear, Sadness, Guilt, Hostility, Shyness, Fatigue, General Positive Affect, Joviality, Self-Assurance, Attentiveness, Serenity, Surprise).

A strong, positive correlation was expected between those who had a victimization history and the General Negative Affect scale. However, the Point-Biserial Correlation revealed that there was little to no relationship between the two variables, $\left(r_{\mathrm{pb}}\right.$ $=.09, p=.626)$. Strong correlations were also expected between those who had a victimization history and the higher-order Sadness, Fear, and Hostility scales. The results revealed a weak relationship between victimization history and Sadness $\left(r_{\mathrm{pb}}=.19, p=\right.$ .307). Because the Fear and Hostility scales violated normality, Mann-Whitney U Tests were conducted to evaluate the differences in responses. Median latencies on the Fear scale for the victimized group and non-victimized group were 11.00 and 11.50, respectively; the groups did not differ significantly $(U=106.00, Z=-.09, p=.932)$. The median latencies on the Hostility scale for the two groups were both approximately 11.00; there was no significant difference between responses $(U=100.00, Z=-.34, p=$ 
.733). Thus, the present results indicated that individuals with victimization histories do not tend to experience significantly higher negative affect, and/or feelings such as sadness, fear or hostility.

It was predicted that weaker correlations (relative to those between victimization history and General Negative Affect) would be observed between those who had a victimization history and the General Positive Affect scale. The correlations between victimization history and General Positive Affect $\left(r_{\mathrm{pb}}=-.02, p=.928\right)$, victimization history and Joviality $\left(r_{\mathrm{pb}}=-.07, p=.735\right)$, as well as between victimization history and Serenity $\left(r_{\mathrm{pb}}=-.13, p=.489\right)$, were all negative and non-significant. With a larger sample size, it may be possible to determine if individuals with victimization histories are truly less likely to report positive emotions. See Table 9 for a complete review of the correlations between the PANAS-X scales and self-reported victimization history. 
Table 9

Correlations Between Affect and Victimization History

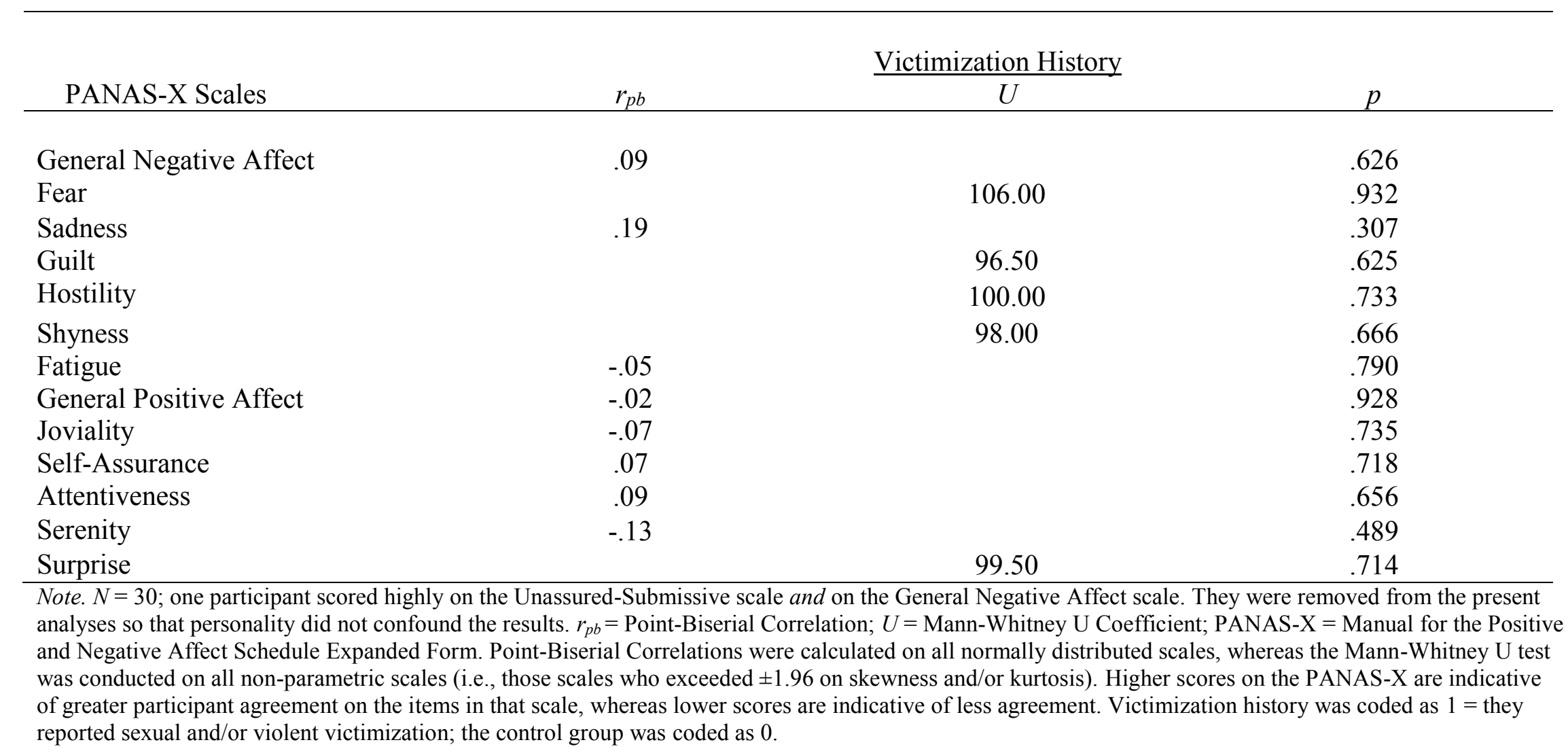


Next, gait pattern (an ordinal variable ranging from $0=$ no vulnerability cues present, to $5=$ all vulnerability cues present) was correlated (using Spearman's RankOrder Correlation) with total scores for each scale measured in the PANAS-X. Recall that a strong, positive correlation was expected between vulnerable-coded gait patterns and the General Negative Affect scale. Strong correlations were also expected between vulnerable-coded gait patterns and the higher-order Fear, Sadness, and Hostility scales. Weaker correlations were expected between vulnerable-coded gait patterns and the positive PANAS-X scales.

Small, positive correlations were observed between gait and the Fear scale $\left(r_{\mathrm{s}}[25]\right.$ $=.29, p=.149)$, as well as between gait and the General Negative Affect scale $\left(r_{\mathrm{s}}[25]=\right.$ $.25, p=.246$ ). None of the correlations reached statistical significance (see Table 10). Given the lack of association between gait and the affect scale variables, as well as between victimization history and the affect scale variables, it was not feasible to conduct a mediated regression similar to that which had been performed for personality. 
Table 10

Correlations Between Affect and Gait

\begin{tabular}{llc}
\hline & \multicolumn{2}{c}{ Gait Pattern } \\
\cline { 2 - 3 } PANAS-X Scale Variables & $r_{\mathrm{s}}$ & $p$ \\
\hline Fear & .29 & .149 \\
Sadness & .09 & .663 \\
Guilt & .12 & .567 \\
Hostility & .02 & .923 \\
Shyness & .18 & .381 \\
Fatigue & -.02 & .920 \\
General Positive Affect & .10 & .631 \\
Joviality & .15 & .453 \\
Self-Assurance & .22 & .267 \\
Attentiveness & .07 & .740 \\
Serenity & .04 & .842 \\
Surprise & .12 & .562 \\
General Negative Affect & .25 & .246 \\
\hline
\end{tabular}

Note. $N=27$; three videos were not useable and a gait vulnerability score could not be computed for them. They were removed from the present analyses. One participant scored highly on both the UnassuredSubmissive scale and on the General Negative Affect scale. They were also removed from the analyses so that personality did not confound the results. PANAS-X = Manual for the Positive and Negative Affect Schedule Expanded Form. $r_{\mathrm{s}}=$ Spearman's Rank-Order Correlation. Higher scores on the PANAS-X are indicative of greater participant agreement on the items in that scale, whereas lower scores are indicative of less agreement. The gait pattern scale variable ranged from $0=$ no vulnerable cues present, to $5=$ all vulnerable cues present.

The effect of time, frequency, severity, and type of victimization, on gait. Several correlations were calculated between the victimization history variables, gait, personality, and affect. As no known literature has examined the specifics of the walkers' victimization history, these analyses were largely exploratory.

Type of victimization. First, a Rank-Biserial Correlation was calculated between self-reported violent victimization (a nominal variable $[0=$ no, $1=$ yes $]$ calculated with eleven violently victimized participants) and gait pattern (an ordinal variable; $0=$ no vulnerability cues present, to $5=$ all vulnerability cues present). The finding was significant, $r_{r b}=.51, p=006$. Walkers who had been violently victimized were significantly more likely to have a vulnerable gait. Interestingly, the relationship between 
sexual victimization (a nominal variable $[0=$ no, $1=$ yes $]$ also calculated with eleven sexually victimized participants) and gait pattern was not significant, $r_{r b}=.32, p=.101^{20}$

Next, the relationship between violent victimization and personality (via the IASR-B5 variables) was examined. A fairly strong, positive, Point-Biserial Correlation was observed between violent victimization and the Openness to Experience scale, $r_{p b}=$ $.51, p=.003$. The association indicates that individuals who have been violently victimized are significantly more likely to report personality traits such as, "philosophical," "individualistic," "literary," and "questioning," among others. A significant, positive relationship was also found between violent victimization and the Assured-Dominant scale, $r_{p b}=.42, p=.018$. Therefore, it appears that individuals who have experienced violent victimization are significantly more likely to see themselves as: "self-assured," "domineering," "firm," and "assertive," among others. See Table 11 for a complete summary of the correlations between violent victimization and the IASR-B5 scales.

\footnotetext{
${ }^{20}$ The current sample was too small $(<30)$ to remove and/or isolate individuals who had been victimized both sexually and violently. Therefore, several individuals who were victims of violent crime, had also been sexually victimized (and vice versa). The findings that differ by "type of victimization" (i.e., sexual versus violent) should thus be interpreted cautiously.
} 
Table 11

Correlations Between Victimization Type and Personality

\begin{tabular}{lccccc}
\hline \multirow{2}{*}{\begin{tabular}{c} 
IASR-B5 Scale \\
\multicolumn{1}{c}{ Variables }
\end{tabular}} & \multicolumn{2}{c}{ Violent Victimization $^{\mathrm{a}}$} & & \multicolumn{2}{c}{ Sexual Victimization $^{\mathrm{b}}$} \\
\cline { 2 - 3 } \cline { 5 - 6 } & $r_{\mathrm{pb}}$ & $p$ & & $r_{\mathrm{pb}}$ & $p$ \\
\hline Unassured-Submissive & -.26 & .165 & & -.00 & .996 \\
Assured-Dominant & .42 & $.018^{*}$ & & -.18 & .341 \\
Gregarious-Extraverted & -.13 & .480 & & -.06 & .763 \\
Warm-Agreeable & -.04 & .841 & & -.06 & .735 \\
Unassuming-Ingenuous & -.24 & .187 & & -.02 & .907 \\
Aloof-Introverted & .05 & .781 & & -.10 & .582 \\
Cold-Hearted & .17 & .362 & & .10 & .606 \\
Arrogant-Calculating & .23 & .224 & & -.17 & .356 \\
Conscientiousness & -.11 & .546 & & .08 & .673 \\
Neuroticism & .15 & .411 & & -.07 & .727 \\
Openness to Experience & .51 & $.003^{* *}$ & & .04 & .849 \\
\hline
\end{tabular}

Note. ${ }^{*} p<.05 .{ }^{* *} p<.01$. IASR-B5 = Revised Interpersonal Adjective Scales Big Five. $r_{\mathrm{pb}}=$ Point-Biserial Correlation. Higher scores on the IASR-B5 are indicative of greater participant agreement on the items in that scale, whereas lower scores are indicative of less agreement. Victimization was a nominally coded variable $(0=$ no, $1=$ yes $)$.

${ }^{a} N=13 .{ }^{\mathrm{b}} N=11$.

There were no significant correlations observed between any of the IASR-B5 scales and sexual victimization (see Table 11). Nearly all of the correlations were negative, except the relationship between Cold-Heartedness and sexual victimization $\left(r_{p b}\right.$ $=.10, p=.606)$, Conscientiousness and sexual victimization $\left(r_{p b}=.08, p=.673\right)$, and Openness to Experience and sexual victimization $\left(r_{p b}=.04, p=.849\right)$.

Point-Biserial Correlations were calculated between violent victimization and the affect scale variables (in the PANAS-X). The only correlation that reached statistical significance was between violent victimization and Attentiveness, $r_{p b}=.36, p=.049$. It appears that individuals who have been violently victimized are significantly more likely to report that they are attentive (e.g., "alert," "concentrating," "determined," etc.). Table 12 outlines the remaining correlations between violent victimization and the PANAS-X scale variables. 
The correlations between sexual victimization and the affect scales did not reach statistical significance. A larger sample may be necessary to determine if an association exists between the type of victimization history one has, and whether or not they are more likely to report certain emotions. Table 12 provides a summary of the correlations between sexual victimization and each of the PANAS-X scales.

Table 12

Correlations Between Victimization Type and Affect

\begin{tabular}{|c|c|c|c|c|c|c|}
\hline \multirow[b]{2}{*}{$\begin{array}{c}\text { PANAS-X Scale } \\
\text { Variables }\end{array}$} & \multicolumn{3}{|c|}{ Violent Victimization $^{\mathrm{a}}$} & \multicolumn{3}{|c|}{ Sexual Victimization $^{b}$} \\
\hline & $r_{\mathrm{pb}}$ & $U$ & $p$ & $r_{\mathrm{pb}}$ & $U$ & $p$ \\
\hline Fear & & 99.00 & .489 & & 94.50 & .528 \\
\hline Sadness & .12 & & .519 & .14 & & .465 \\
\hline Guilt & & 92.50 & .332 & & 109.00 & .984 \\
\hline Hostility & & 105.00 & .650 & & 107.50 & .919 \\
\hline Shyness & & 105.50 & .650 & & 85.00 & .317 \\
\hline Fatigue & .03 & & .877 & -.14 & & .443 \\
\hline General Positive & .14 & & .460 & .03 & & .889 \\
\hline \multicolumn{7}{|l|}{ Affect } \\
\hline Joviality & .02 & & .923 & -.02 & & .913 \\
\hline Self-Assurance & .27 & & .147 & -.01 & & .961 \\
\hline Attentiveness & .36 & & $.049 *$ & .14 & & .444 \\
\hline Serenity & .04 & & .814 & -.19 & & .306 \\
\hline Surprise & & 116.00 & .984 & & 102.00 & .761 \\
\hline $\begin{array}{l}\text { General Negative } \\
\text { Affect }\end{array}$ & .17 & & .359 & .09 & & .640 \\
\hline $\begin{array}{l}\text { Note. }{ }^{*} p<.05 . \text { PANAS- } \\
\text { Point-Biserial Correlation } \\
\text { indicative of greater part } \\
\text { less agreement. Victimiz } \\
{ }^{a} N=13 .{ }^{b} N=11 .\end{array}$ & Tanual & the Positi & $\begin{array}{l}\text { d Negati } \\
\text { icient. Hi } \\
\text { in that sc } \\
\text { ariable }(0\end{array}$ & $\begin{array}{l}\text { Schedu } \\
\text { es on th } \\
\text { eas low } \\
\text { yes). }\end{array}$ & $\begin{array}{l}\text { panded } \mathrm{Fc} \\
\mathrm{NAS}-\mathrm{X} \text { a } \\
\text { ores are in }\end{array}$ & $\begin{array}{l}\mathrm{pb}= \\
\text { ive of }\end{array}$ \\
\hline
\end{tabular}

Time frame of victimization. If a participant indicated that they had been violently and/or sexually victimized, then they were provided a set of additional questions pertaining to each experience. First, they were asked to indicate when they had been victimized (on a 5-point scale ranging from $1=$ very recently [i.e., within the last few weeks], to 5 = very long ago [i.e., 5+ years ago]). Spearman's Rank-Order Correlations 
were calculated to examine whether time frame of victimization was associated with gait vulnerability, personality, and/or affect.

No relationship was observed between the time frame of one's violent victimization and the vulnerability score of their gait pattern, $r_{\mathrm{s}}(9)=-.43, p=.189$. Similarly, the relationship between the time frame of one's sexual victimization and their gait was not significant, $r_{\mathrm{s}}(9)=-.23, p=.505$.

There was a significant relationship observed between the time frame of one's violent victimization and the Warm-Agreeable scale in the IASR-B5, $r_{\mathrm{s}}(11)=-.79, p=$ .001 . There was also a significant correlation between the time frame of violent victimization and the Cold-Hearted scale $r_{\mathrm{s}}(11)=.66, p=.014$. It appears that individuals who were violently victimized long ago (i.e., further back in their past), were significantly more likely to report personality traits consistent with cold-heartedness (e.g., "ruthless," "cruel," "unsympathetic," and "iron-hearted," among others), and significantly less likely to report warm-agreeable type traits (e.g., "kind," “accommodating," "tender," and "gentle-hearted," among others). See Table 13 for a review of the remaining correlations between time frame of violent victimization and the personality scales.

Spearman Correlations were also calculated between the time frame of one's sexual victimization and their reported personality characteristics. A significant relationship was observed between the time frame of one's sexual victimization experience and the Assured-Dominant scale in the IASR-B5, $r_{\mathrm{s}}(9)=.65, p=.032$. The more time that has passed since one's sexual victimization experience, the more dominant individuals report themselves as being. The relationship between timing of 
sexual victimization and the Arrogant-Calculating scale was also marginally significant, $r_{\mathrm{s}}(9)=.59, p=.056$. Thus, it appears that individuals who experienced sexual victimization longer ago may be more likely to present with arrogant personality features (e.g., "cocky," "sly," "boastful," and "cunning," among others). The other correlations were not significant at $p<.05$. See Table 13 for an outline of the remaining correlations. 
Table 13

Correlations Between Time Frame of Victimization and Personality

\begin{tabular}{|c|c|c|c|c|}
\hline \multirow[b]{2}{*}{ IASR-B5 Scale Variables } & \multicolumn{2}{|c|}{ Time Frame of Violent Victimization ${ }^{\mathrm{a}}$} & \multicolumn{2}{|c|}{ Time Frame of Sexual Victimization $^{b}$} \\
\hline & $r_{\mathrm{S}}$ & $p$ & $r_{\mathrm{S}}$ & $p$ \\
\hline Unassured-Submissive & .20 & .524 & -.48 & .137 \\
\hline Assured-Dominant & -.33 & .273 & .65 & $.032 *$ \\
\hline Gregarious-Extraverted & -.44 & .136 & -.22 & .524 \\
\hline Warm-Agreeable & -.79 & $.001 * *$ & -.46 & .152 \\
\hline Unassuming-Ingenuous & -.38 & .199 & -.05 & .875 \\
\hline Aloof-Introverted & .50 & .084 & .02 & .943 \\
\hline Cold-Hearted & .66 & $.014^{* *}$ & .39 & .236 \\
\hline Arrogant-Calculating & .43 & .143 & .59 & .056 \\
\hline Conscientiousness & .34 & .259 & .14 & .688 \\
\hline Neuroticism & -.00 & .992 & .38 & .256 \\
\hline Openness to Experience & -.23 & .451 & .07 & .841 \\
\hline
\end{tabular}
are indicative of greater participant agreement on the items in that scale, whereas lower scores are indicative of less agreement. Time frame of victimization was measured on a 5 -point scale ranging from $1=$ very recently (i.e., within the last few weeks), to $5=$ very long ago (i.e., $5+$ years ago).

${ }^{a} N=13 .{ }^{\mathrm{b}} N=11$. 
There were no significant correlations observed between the time frame of one's violent victimization and the affect scale variables (see Table 14). A larger sample size is necessary to determine whether a relationship exists between the timing of an individual's violent victimization and their emotional wellbeing.

In regards to sexual victimization, there was a significant association between the time frame of one's sexual victimization and the Shyness scale in the PANAS-X, $r_{\mathrm{s}}(9)=$ $-.61, p=.047$. The correlation indicates that individuals who were sexually victimized more recently tend to report traits characteristic of Shyness (e.g., "sheepish," "timid," etc.). In contrast, individuals who were sexually victimized long ago are less likely to report that they are shy. There was also a significant association between the time frame of one's sexual victimization and the Attentiveness scale, $r_{\mathrm{s}}(9)=.62, p=.044$. Thus, individuals who were sexually victimized a long time ago are more likely to report that they are attentive (e.g., "alert," “concentrating," etc.). See Table 14 for a review of the remaining correlations. 
Table 14

Correlations Between Time Frame of Victimization and Affect

\begin{tabular}{|c|c|c|c|c|}
\hline \multirow[b]{2}{*}{ PANAS-X Scale Variables } & \multicolumn{2}{|c|}{ Time Frame of Violent Victimization $^{\mathrm{a}}$} & \multicolumn{2}{|c|}{ Time Frame of Sexual Victimization ${ }^{b}$} \\
\hline & $r_{\mathrm{S}}$ & $p$ & $r_{\mathrm{S}}$ & $p$ \\
\hline Fear & -.21 & .483 & -.08 & .807 \\
\hline Sadness & .02 & .942 & -.41 & .207 \\
\hline Guilt & -.23 & .443 & -.26 & .444 \\
\hline Hostility & -.16 & .611 & -.32 & .334 \\
\hline Shyness & -.03 & .915 & -.61 & $.047 *$ \\
\hline Fatigue & .20 & .509 & -.33 & .315 \\
\hline General Positive Affect & -.20 & .517 & .44 & .175 \\
\hline Joviality & -.38 & .206 & .12 & .720 \\
\hline Self-Assurance & -.39 & .189 & .34 & .308 \\
\hline Attentiveness & .38 & .198 & .62 & $.044 *$ \\
\hline Serenity & .18 & .566 & .13 & .694 \\
\hline Surprise & -.27 & .376 & -.27 & .421 \\
\hline General Negative Affect & -.26 & .385 & -.32 & .336 \\
\hline
\end{tabular}

General Negative Affect

Note $* p<.05$. PANAS-X = Manual for the Positive and Negative Affect Schedule Expanded Form. $r_{\mathrm{s}}=$ Spearman's Rank-0 victimization was measured on a 5 -point scale ranging from $1=$ very recently (i.e., within the last few weeks), to $5=$ very long ago (i.e., $5+$ years ago). ${ }^{a} N=13 .{ }^{\mathrm{b}} N=11$. 
Frequency of victimization. Next, participants were asked to report the number of times that they had experienced each type of victimization; the scale variable ranged from "once" to "5+ times." Frequency of violent victimization was negatively correlated (using Spearman's Rank-Order Correlations) with gait pattern, $r_{\mathrm{s}}(9)=-.22, p=.514$, but the relationship was not significant. Similarly, the relationship between the frequency of sexual victimization and gait was not significant, $r_{\mathrm{s}}(9)=.39, p=.242$. Although less overlap among the victimization categories (i.e., removing individuals who have been victimized both sexually and violently) is required to confirm the lack of association, it appears that multiple versus singular victimization has little relationship with the vulnerability level of one's gait pattern.

For exploratory purposes, frequency of victimization was also examined in relation to personality and the affect scales using Spearman's Rank-Order Correlation. None of the correlations between frequency of violent victimization and personality were significant, nor were those between frequency of sexual victimization and personality (see Table 15). 
Table 15

Correlations Between the Frequency of Victimization and Personality

\begin{tabular}{|c|c|c|c|c|}
\hline \multirow[b]{2}{*}{ IASR-B5 Scale Variables } & \multicolumn{2}{|c|}{ Frequency of Violent Victimization ${ }^{\mathrm{a}}$} & \multicolumn{2}{|c|}{ Frequency of Sexual Victimization } \\
\hline & $r_{\mathrm{s}}$ & $p$ & $r_{\mathrm{S}}$ & $p$ \\
\hline Assured-Dominant & .05 & .878 & .02 & .943 \\
\hline Warm-Agreeable & -.21 & .489 & .38 & .248 \\
\hline Unassuming-Ingenuous & .07 & .823 & .40 & .221 \\
\hline Aloof-Introverted & .24 & .439 & -.19 & .579 \\
\hline Conscientiousness & -.30 & .320 & -.42 & .198 \\
\hline Neuroticism & .12 & .697 & .15 & .660 \\
\hline Openness to Experience & -.06 & .853 & .35 & .294 \\
\hline
\end{tabular}

Note. IASR-B5 = Revised Interpersonal Adjective Scales Big Five. $r_{\mathrm{s}}=$ Spearman's Rank-Order Correlation. Higher scores on the IASR-B5 are indicative of greater participant agreement on the items in that scale, whereas lower scores are indicative of less agreement. Frequency of victimization was a scale variable that ranged from "once" to " $5+$ times."

${ }^{\mathrm{a}} N=13 \cdot{ }^{\mathrm{b}} N=11$ 
None of the correlations between frequency of violent victimization and affect reached significance (see Table 16). Interestingly, however, there was a significant relationship observed between the frequency with which one has experienced sexual victimization and the Guilt scale in the PANAS-X, $r_{\mathrm{s}}(9)=.69, p=.020$. Individuals who had been sexually victimized multiple times were more likely to report higher levels of guilt (adjectives on the Guilt scale include: "disgusted with self," "blameworthy," and "ashamed," among others). There was also a significant relationship found between frequency of sexual victimization and Fear (adjectives on the Fear scale include: "scared," "nervous," shaky," etc.), $r_{\mathrm{s}}(9)=.66, p=.027$. Table 16 provides the full set of correlations between frequency of sexual victimization and the PANAS-X scales. 
Table 16

Correlations Between the Frequency of Victimization and Affect

\begin{tabular}{|c|c|c|c|c|}
\hline PANAS-X Scale Variables & \multicolumn{2}{|c|}{ Frequency of Violent Victimization $^{\mathrm{a}}$} & \multicolumn{2}{|c|}{ Frequency of Sexual Victimization } \\
\hline Sadness & -.02 & .948 & .51 & .108 \\
\hline Hostility & .17 & .578 & -.10 & .773 \\
\hline Shyness & -.16 & .601 & .04 & .909 \\
\hline Fatigue & -.26 & .385 & .10 & .760 \\
\hline Self-Assurance & -.10 & .737 & .03 & .927 \\
\hline Attentiveness & -.12 & .702 & -.15 & .657 \\
\hline Serenity & -.20 & .510 & -.35 & .294 \\
\hline Surprise & .03 & .924 & .42 & .199 \\
\hline General Negative Affect & .39 & .193 & .51 & .112 \\
\hline
\end{tabular}

Note. ${ }^{*} p<.05$. PANAS-X $=$ Manual for the Positive and Negative Affect Schedule Expanded Form. $r_{\mathrm{s}}=$ Spearman's Rank-Order Correlation. Higher scores on the PANAS-X are indicative of greater participant agreement on the items in that scale, whereas lower scores are indicative of less agreement. Frequency of victimization was a scale variable that ranged from "once" to " $5+$ times."

${ }^{\mathrm{a}} N=13 \cdot{ }^{\mathrm{b}} N=11$. 
Severity of victimization. The final questions asked in the Victimization History Questionnaire Extended pertained to the subjective severity of the event. Rank-Biserial Correlations were calculated to examine the relationship between the severity of violent/sexual victimization and gait pattern, when severity was measured as a dichotomous (yes $=0$, no $=1$ ) variable. None of the correlations were significant when violent victimization was considered. In contrast, individuals who perceived themselves to be in danger at the time that they were sexually victimized (i.e., they responded "yes") were more likely to exhibit a vulnerable gait pattern, $r_{r b}=-.83, p=002$. This finding may indicate that more severe sexual victimization experiences impact the perceived vulnerability of one's walk. Table 17 summarizes the findings.

Table 17

Correlations Between Severity of Victimization Measured Nominally, and Gait

\begin{tabular}{lcc}
\hline $\begin{array}{l}\text { Dichotomous Severity } \\
\text { Questions }\end{array}$ & $r_{\mathrm{rb}}$ & Gait Pattern \\
\hline Violent Victimization & & \\
$\quad$ Feelings of Danger & .19 & .572 \\
$\quad$ Seriously Injured & -.13 & .704 \\
$\quad$ Feelings of Fear & -.12 & .726 \\
Sexual Victimization & -.83 & $.002^{*}$ \\
$\quad$ Feelings of Danger & .00 & - \\
Seriously Injured & .00 & - \\
Feelings of Fear & & \\
\hline
\end{tabular}

Note. $N=11 .{ }^{*} p<.01 . r_{\mathrm{rb}}=$ Rank-Biserial Correlation. Victimization severity questions were asked in a yes $/$ no format; yes $=0$, no $=1$. The gait pattern scale variable ranged from $0=$ no vulnerable cues present, to $5=$ all vulnerable cues present.

Point-Biserial Correlations were calculated between each of the personality scales in the IASR-B5 and the dichotomous severity items (i.e., one's feeling of danger [yes/no], their feelings of fear [yes/no], and the presence of serious injury [yes/no]). 
There was a significant association between one's feeling of fear at the time that they were violently victimized and the Gregarious-Extraverted scale, $r_{p b}=-.60, p=.031$. That is, individuals who indicated that they were afraid at the time when they were violently victimized were more likely to report traits consistent with Gregarious-Extraversion (i.e., adjectives such as "outgoing," “enthusiastic," etc.). There was also a significant relationship observed between feelings of fear during violent victimization and the Warm-Agreeable scale $r_{p b}=-.74, p=.004$. The association indicated that individuals who experienced greater fear at the time that they were violently victimized were more likely to self-report Warm-Agreeable type traits (e.g., "tender," "accommodating," etc.). A summary of the remaining correlations between the severity of violent victimization and personality is outlined in Table 18 .

In terms of sexual victimization, there was a significant relationship observed between Conscientiousness and feelings of fear, $r_{p b}=.63, p=.039$. Strangely, the finding indicated that individuals who were more conscientious (e.g., "planful," "systematic," etc.) tended to report that they were not afraid at the time when they were sexually victimized. It is possible that these reports represent instances of acquaintance rape and/or sexual victimization by a known other; relational abuse may be less likely to produce the same feelings of fear that stranger assault would. Additional research is required to better understand the relationship between victim personality traits (e.g., conscientiousness) and the likelihood of one experiencing stranger versus domestic assault.

There was also a significant correlation between one's feeling of danger at the time that they were sexually victimized and the Cold-Heartedness scale, $r_{p b}=.66, p=$ 
.029. It appears that individuals who did not feel as though they were in danger at the time when they were sexually victimized were more likely to self-report Cold-Hearted type traits (e.g., "warmthless," "cruel," etc.). It is unclear whether or not cold-hearted traits act as a defence mechanism for the individuals in the present sample (i.e., if they developed such traits after the event), or if they were present before the victimization occurred.

Several correlations between the severity of sexual victimization and personality were marginally significant (see Table 18). For example, individuals who reported that they felt as though they were in danger at the time when they were sexually victimized were more likely to self-report Gregarious-Extraverted traits, $r_{p b}=-.60, p=.050$. Similarly, individuals who reported that they felt afraid when they were sexually victimized were more likely to self-report traits consistent with the UnassuredSubmissive scale, $r_{p b}=-.59, p=.054$ 
Table 18

Correlations Between Severity of Victimization Measured Nominally, and Personality

\begin{tabular}{|c|c|c|c|c|c|c|c|c|c|c|c|c|}
\hline \multirow{3}{*}{ IASR-B5 Scale Variables } & & \multicolumn{5}{|c|}{ Violent Victimization $^{\mathrm{a}}$} & \multicolumn{6}{|c|}{ Sexual Victimization $^{\mathrm{b}}$} \\
\hline & \multicolumn{2}{|c|}{$\begin{array}{l}\text { Feelings } \\
\text { of Danger }\end{array}$} & \multicolumn{2}{|c|}{$\begin{array}{l}\text { Seriously } \\
\text { Injured }\end{array}$} & \multicolumn{2}{|c|}{$\begin{array}{l}\text { Feelings of } \\
\text { Fear }\end{array}$} & \multicolumn{2}{|c|}{$\begin{array}{c}\text { Feelings of } \\
\text { Danger }\end{array}$} & \multicolumn{2}{|c|}{$\begin{array}{l}\text { Seriously } \\
\text { Injured }\end{array}$} & \multicolumn{2}{|c|}{$\begin{array}{c}\text { Feelings of } \\
\text { Fear }\end{array}$} \\
\hline & $r_{p b}$ & $p$ & $r_{p b}$ & $p$ & $r_{p b}$ & $p$ & $r_{p b}$ & $p$ & $r_{p b}$ & $p$ & $r_{p b}$ & $p$ \\
\hline Conscientiousness & .09 & .781 & .11 & .733 & .30 & .314 & .33 & .319 & .15 & .653 & .63 & $.039 *$ \\
\hline Neuroticism & -.22 & .471 & -.34 & .258 & -.05 & .885 & .06 & .863 & -.23 & .503 & .38 & .248 \\
\hline Openness to Experience & -.11 & .715 & -.34 & .253 & .39 & .191 & -.21 & .542 & -.29 & .391 & -.23 & .495 \\
\hline Unassured-Submissive & .10 & .749 & .36 & .229 & -.35 & .235 & .16 & .634 & .17 & .613 & -.59 & $.054^{*}$ \\
\hline Assured-Dominant & .18 & .557 & .28 & .356 & .03 & .936 & -.10 & .779 & -.29 & .394 & .35 & .287 \\
\hline Gregarious-Extraverted & -.07 & .833 & .01 & .982 & -.60 & $.031 *$ & -.60 & $.050 *$ & .05 & .889 & -.12 & .732 \\
\hline Warm-Agreeable & .02 & .937 & .14 & .641 & -.74 & $.004 * *$ & -.58 & .063 & -.05 & .896 & -.49 & .124 \\
\hline Unassuming-Ingenuous & .12 & .698 & -.03 & .933 & -.47 & .106 & -.29 & .387 & -.20 & .566 & -.15 & .663 \\
\hline Aloof-Introverted & .28 & .360 & .35 & .240 & .11 & .722 & .56 & .075 & -.13 & .698 & -.20 & .547 \\
\hline Cold-Hearted & .01 & .968 & .07 & .832 & .34 & .257 & .66 & $.029 *$ & .00 & - & .39 & .235 \\
\hline Arrogant-Calculating & .13 & .671 & .35 & .242 & .30 & .314 & .44 & .172 & -.17 & .612 & -.08 & .824 \\
\hline
\end{tabular}


Point-Biserial Correlations were also calculated between the nominal severity of violent victimization items and the PANAS-X affect scales. A Mann-Whitney U test demonstrated that there was a significant difference between those who were injured (Mean rank $=11.17$ ) and those were not injured (Mean rank $=5.75)$ on the Fear scale, $U=2.50, Z=-2.15, p=.028$. A marginally significant relationship was also observed between the General Negative Affect and the Seriously Injured item, $r_{p b}=-.55, p=.051$. Table 19 provides a summary of the correlations between affect and the severity of one's violent victimization history. 
Table 19

Correlations Between Severity of Violent Victimization Measured Nominally, and Affect

\begin{tabular}{|c|c|c|c|c|c|c|c|c|c|}
\hline \multirow{2}{*}{$\begin{array}{c}\text { PANAS-X Scale } \\
\text { Variables }\end{array}$} & \multicolumn{3}{|c|}{ Feelings of Danger } & \multicolumn{3}{|c|}{$\underline{\text { Seriously Injured }}$} & \multicolumn{3}{|c|}{$\underline{\text { Feelings of Fear }}$} \\
\hline & $r_{p b}$ & $U$ & $p$ & $r_{p b}$ & $U$ & $p$ & $r_{p b}$ & $U$ & $p$ \\
\hline General Negative Affect & -.14 & & .650 & -.55 & & $.051 *$ & .01 & & .969 \\
\hline Fear & & 20.00 & .945 & & 2.50 & $.028^{*}$ & & 8.00 & .641 \\
\hline Sadness & -.38 & & .195 & -.50 & & .082 & .42 & & .148 \\
\hline Guilt & & 9.00 & .101 & & 7.00 & .217 & & 10.50 & .923 \\
\hline Hostility & & 20.00 & .945 & & 14.00 & .937 & & 7.00 & .513 \\
\hline Shyness & & 20.00 & .945 & & 12.00 & .692 & & 9.50 & .769 \\
\hline Fatigue & -.19 & & .532 & .06 & & .857 & .29 & & .330 \\
\hline General Positive Affect & -.03 & & .928 & -.02 & & .957 & -.15 & & .615 \\
\hline Joviality & .17 & & .585 & .28 & & .357 & -.46 & & .110 \\
\hline Self-Assurance & .04 & & .904 & -.04 & & .910 & -.15 & & .625 \\
\hline Attentiveness & -.04 & & .892 & .16 & & .594 & .31 & & .308 \\
\hline Serenity & .11 & & .719 & .32 & & .280 & -.25 & & .405 \\
\hline Surprise & & 20.50 & .945 & & 13.00 & .811 & & 9.00 & .769 \\
\hline
\end{tabular}


In terms of sexual victimization, a significant relationship was observed between the Seriously Injured severity item and the Self-Assurance scale, $r_{p b}=-.64, p=.035$. Thus, individuals who scored higher on items such as "confident," "bold," "fearless," and "daring," were more likely to indicate that they had been seriously injured at the time when they were sexually victimized. The remaining correlations are provided in Table 20 below. 
Table 20

Correlations Between Severity of Sexual Victimization Measured Nominally, and Affect

\begin{tabular}{|c|c|c|c|c|c|c|c|c|c|}
\hline \multirow{2}{*}{$\begin{array}{c}\text { PANAS-X Scale } \\
\text { Variables }\end{array}$} & \multicolumn{3}{|c|}{$\underline{\text { Feelings of Danger }}$} & \multicolumn{3}{|c|}{$\underline{\text { Seriously Injured }}$} & \multicolumn{3}{|c|}{ Feelings of Fear } \\
\hline & $r_{p b}$ & $U$ & $p$ & $r_{p b}$ & $U$ & $p$ & $r_{p b}$ & $U$ & $p$ \\
\hline General Negative Affect & -.48 & & .131 & .03 & & .921 & -.08 & & .811 \\
\hline Fear & & 5.50 & .194 & & 2.50 & .600 & & 15.00 & - \\
\hline Sadness & -.24 & & .484 & -.05 & & .895 & -.27 & & .426 \\
\hline Guilt & & 5.50 & .194 & & 4.00 & - & & 13.00 & .792 \\
\hline Hostility & & 3.50 & .085 & & 4.50 & - & & 15.00 & - \\
\hline Shyness & & 10.00 & .776 & & 1.00 & .400 & & 6.00 & .126 \\
\hline Fatigue & .09 & & .797 & .34 & & .311 & .06 & & .858 \\
\hline General Positive Affect & -.11 & & .746 & -.48 & & .134 & -.05 & & .882 \\
\hline Joviality & -.16 & & .647 & -.08 & & .808 & .01 & & .985 \\
\hline Self-Assurance & -.13 & & .702 & -.64 & & $.035^{*}$ & -.39 & & .237 \\
\hline Attentiveness & .16 & & .626 & -.53 & & .095 & .12 & & .736 \\
\hline Serenity & .14 & & .690 & -.45 & & .169 & -.52 & & .101 \\
\hline Surprise & & 4.50 & .133 & & .500 & .200 & & 6.00 & .126 \\
\hline
\end{tabular}

Note. $N=11 . r_{\mathrm{pb}}=$ Point-Biserial Correlation. $U=$ Mann-Whitney U Coefficient. ${ }^{*} p<.05$. Victimization severity questions were asked in a yes/no format; yes $=$ 0, no $=1$. Higher scores on the PANAS-X are indicative of greater participant agreement on the items in that scale, whereas lower scores are indicative of less agreement. 
There were several questions included in the Victimization History Questionnaire Extended that measured severity on a scale (e.g., "Rank the emotional severity of your [sexual] victimization experience(s) on the 5-point scale;" $1=$ not severe, $5=$ extremely severe). For exploratory purposes, each of the ordinal severity items was correlated with the gait vulnerability ratings, the IASR-B5 personality scales, and the PANAS-X affect scales.

First, Spearman's Rank-Order Correlations were calculated to examine the relationship between the severity of violent/sexual victimization and gait. None of the correlations were significant when violent victimization was considered. In contrast, there was a significant correlation observed was between feelings of distress during sexual victimization and gait, $r_{s}(9)=.61, p=.046$. Individuals who experienced more distress following their sexual victimization experience(s) were more likely to exhibit vulnerable gait features, providing some evidence that more (subjectively) severe sexual victimization experience(s) may impact the perceived vulnerability of one's gait. Table 21 outlines the remaining correlations between gait pattern and the ordinal severity items. 
Table 21

Correlations Between Severity of Victimization Measured on an Ordinal Scale, and Gait

\begin{tabular}{llll}
\hline \multirow{2}{*}{ Scaled Severity Questions } & \multicolumn{3}{c}{ Gait Pattern } \\
\cline { 2 - 3 } & $r_{\mathrm{s}}$ & $p$ & $p$ \\
\hline
\end{tabular}

Violent Victimization

Rating of Distress

Length of Time Bothered

Extent Bothered

Rating of Emotional Severity ${ }^{\mathrm{a}}$

Rating of Psych. Severity ${ }^{a}$

.34

.302

Rating of Physical Severity ${ }^{a}$

Sexual Victimization

Rating of Distress

Length of Time Bothered

Extent Bothered

Rating of Emotional Severity ${ }^{\mathrm{a}}$

Note. $N=11 .{ }^{*} p<.05 . r_{\mathrm{s}}=$ Spearman's Rank-Order Correlation. The "Rating of Distress" variable ranged from $0=$ none, to $3=$ severe. The "Length of Time Bothered" variable ranged from $0=$ not at all, to $3=\mathrm{a}$ month or more. The "Extent Bothered" variable ranged from $0=$ not at all, to $4=$ very much. The gait pattern variable ranged from $0=$ no vulnerable cues present, to $5=$ all vulnerable cues present.

${ }^{\text {a }}$ The variable ranged from $1=$ not severe, to $5=$ extremely severe.

Next, Spearman's Rank-Order Correlations were calculated between the severity of one's violent victimization and the IASR-B5 personality scales. Several correlations were significant. Individuals with higher ratings of distress were significantly less likely to self-report submissive personality traits, $r_{\mathrm{s}}(11)=-.68, p=.010$. Individuals who experienced more distress were also significantly less likely to report traits consistent with the Aloof-Introverted scale (e.g., "antisocial," "distant," etc.), $r_{\mathrm{s}}(11)=-.72, p=$ .005. Lastly, a significant association was observed between Neuroticism and ratings of distress, $r_{\mathrm{S}}(11)=.62, p=.023$.

Participants were asked to indicate the length of time that they were bothered by the violent event and the extent to which they remembered being bothered by it. There 
was a significant association between the length of time that individuals were bothered by the experience and the Gregarious-Extraverted scale $\left(r_{\mathrm{s}}[11]=.58, p=.037\right)$, as well as between the length of time bothered and the Warm-Agreeable scale $\left(r_{\mathrm{s}}[11]=.65, p=\right.$ .016). Therefore, individuals who considered themselves to be "sympathetic" and "kind," (i.e., traits consistent with the Warm-Agreeable scale) tended to be bothered by their violent victimization experience for a longer length of time. Similarly, those who considered themselves to be "friendly" and "perky," (traits consistent with the Gregarious-Extraverted scale) also reported that they were bothered for a longer period of time.

Not surprisingly, those who indicated that they exhibited Warm-Agreeable traits were significantly more likely to be bothered by the experience $\left(r_{\mathrm{s}}[11]=.73, p=.005\right)$, as were those who scored highly on the Unassuming-Ingenuous scale $\left(r_{\mathrm{s}}[11]=.74, p=\right.$ .004). Individuals who were least bothered by the experience scored highly on the ColdHearted scale $\left(r_{\mathrm{s}}[11]=-.56, p=.045\right)$ and on the Arrogant-Calculating scale $\left(r_{\mathrm{s}}[11]=-\right.$ $.56, p=.046)$.

The final questions in the Victimization History Questionnaire Extended asked participants to indicate (on a scale ranging from 1 to 5 ) how emotionally, psychologically, and physically severe they perceived their violent victimization experience to be. Individuals who considered themselves to be Unassuming-Ingenuous (i.e., "unargumentative," "boastless," etc.), were significantly more likely to rate the emotional severity of their violent victimization experience as more extreme, $r_{\mathrm{s}}(11)=$ $.66, p=.015$. 
Individuals who perceived their violent victimization experience to be psychologically severe were significantly more likely to report traits consistent with the Warm-Agreeable $\left(r_{\mathrm{s}}[11]=.70, p=.008\right)$, Unassuming-Ingenuous $\left(r_{\mathrm{s}}[11]=.73, p=\right.$ $.005)$, and Conscientious $\left(r_{\mathrm{s}}[11]=.68, p=.011\right)$ scales. Alternatively, those who scored highly on Cold-Heartedness perceived their violent victimization to be less psychologically severe $\left(r_{\mathrm{s}}[11]=-.60, p=.032\right)$. Table 22 outlines the remaining correlations between the personality scales and the ordinal severity of violent victimization questionnaire items. 
Table 22

Correlations Between Severity of Violent Victimization Measured on an Ordinal Scale, and Personality

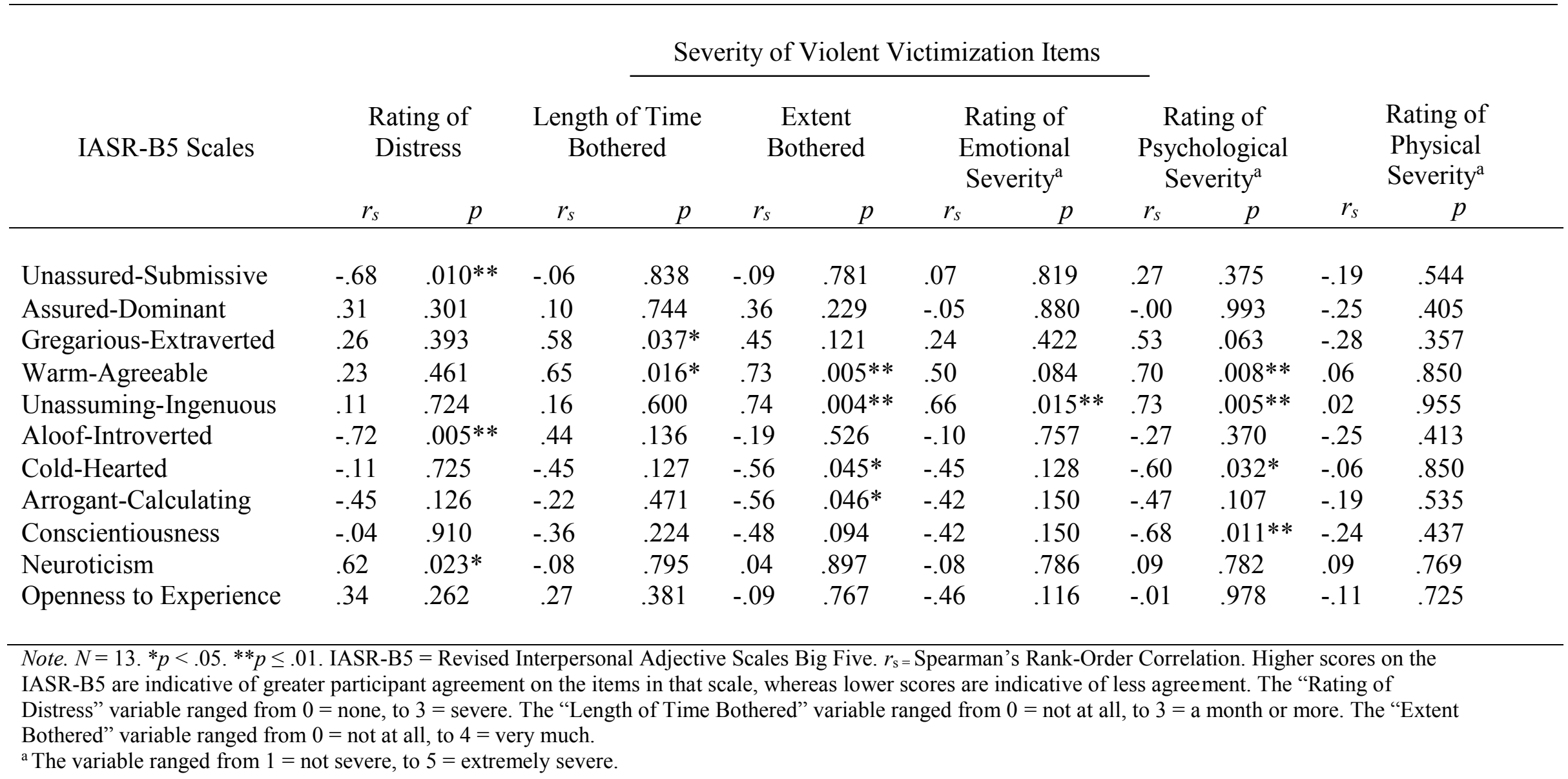


Spearman's Rank-Order Correlations were also conducted to examine the relationship between the severity of the participants' sexual victimization experience(s) and their personality features. None of the correlations were significant, but several marginally significant findings are highlighted in Table 23. 
Table 23

Correlations Between Severity of Sexual Victimization Measured on an Ordinal Scale, and Personality

\begin{tabular}{|c|c|c|c|c|c|c|c|c|c|c|c|c|}
\hline \multirow{3}{*}{ IASR-B5 Scales } & & & & \multicolumn{6}{|c|}{ Severity of Sexual Victimization Items } & & & \\
\hline & \multicolumn{2}{|c|}{$\begin{array}{l}\text { Rating of } \\
\text { Distress }\end{array}$} & \multicolumn{2}{|c|}{$\begin{array}{l}\text { Length of Time } \\
\text { Bothered }\end{array}$} & \multicolumn{2}{|c|}{$\begin{array}{c}\text { Extent } \\
\text { Bothered }\end{array}$} & \multicolumn{2}{|c|}{$\begin{array}{l}\text { Rating of } \\
\text { Emotional } \\
\text { Severity }^{\mathrm{a}}\end{array}$} & \multicolumn{2}{|c|}{$\begin{array}{c}\text { Rating of } \\
\text { Psychological } \\
\text { Severity }^{\mathrm{a}}\end{array}$} & \multicolumn{2}{|c|}{$\begin{array}{l}\text { Rating of } \\
\text { Physical } \\
\text { Severity }^{\mathrm{a}}\end{array}$} \\
\hline & $r_{s}$ & $p$ & $r_{s}$ & $p$ & $r_{s}$ & $p$ & $r_{s}$ & $p$ & $r_{s}$ & $p$ & $r_{s}$ & $p$ \\
\hline Gregarious-Extraverted & .34 & .312 & -.52 & .098 & .02 & .966 & .03 & .927 & .14 & .682 & .17 & .618 \\
\hline Warm-Agreeable & .57 & .070 & -.24 & .473 & .16 & .646 & -.02 & .960 & .18 & .599 & .21 & .542 \\
\hline Unassuming-Ingenuous & .26 & .437 & -.11 & .748 & .16 & .634 & -.17 & .625 & -.04 & .913 & -.14 & .693 \\
\hline Aloof-Introverted & -.22 & .509 & .26 & .442 & -.24 & .486 & -.33 & .319 & -.46 & .154 & -.34 & .308 \\
\hline Cold-Hearted & -.49 & .130 & .28 & .405 & -.03 & .931 & .09 & .784 & -.16 & .632 & .00 & .994 \\
\hline
\end{tabular}

Note. $N=11 .{ }^{*}$ marginal significance. IASR-B5 = Revised Interpersonal Adjective Scales Big Five. $r_{\mathrm{s}}=$ Spearman's Rank-Order Correlation. Higher scores on the IASR-B5 are indicative of greater participant agreement on the items in that scale, whereas lower scores are indicative of less agreement. The "Rating of

Distress" variable ranged from $0=$ none, to $3=$ severe. The "Length of Time Bothered" variable ranged from $0=$ not at all, to $3=$ a month or more. The "Extent Bothered" variable ranged from $0=$ not at all, to $4=$ very much.

${ }^{\text {a }}$ The variable ranged from $1=$ not severe, to $5=$ extremely severe. 
Spearman's Rank-Order Correlations were calculated between affect and the ordinal severity of violent victimization items. Individuals who considered themselves to be shy were significantly less likely to report feelings of distress, $r_{\mathrm{s}}(11)=-.57, p=.044$. Similarly, those who reported more serenity were significantly less likely to report feelings of distress, $r_{\mathrm{s}}(11)=-.72, p=.006$.

Ironically, there was a significantly negative association between Fatigue and the length of time that individuals were bothered by their violent victimization experience(s), $r_{\mathrm{s}}(11)=-.59, p=.034$. The correlation indicated that individuals who reported higher levels of fatigue tended to be bothered for shorter periods of time. There was also a significant relationship between Joviality and the length of time bothered, $r_{\mathrm{s}}(11)=.62, p$ $=.025$. It appears that individuals who perceived themselves to be "happy" and "cheerful," were more likely to have been bothered for a longer period of time by their violent victimization experience(s). Lastly, individuals with higher scores on the SelfAssurance scale were significantly more likely to be bothered by their violent victimization for a greater period of time, $r_{\mathrm{s}}(11)=.65, p=.016$.

There was a significant correlation observed between Attentiveness and the extent to which individuals reported being bothered by their violent victimization, $r_{\mathrm{s}}(11)=-.58$, $p=.038$. Individuals who reported that they were attentive were significantly less bothered by the event. Those with higher levels of attentiveness also rated the Emotional Severity of their violent victimization lower, $r_{\mathrm{s}}(11)=-.57, p=.044$. Finally, individuals who perceived their violent victimization to be physically severe were significantly more likely to exhibit sadness $\left(r_{\mathrm{S}}[11]=.55, p=.050\right)$ and guilt $\left(r_{\mathrm{s}}[11]=.64, p=.019\right)$. See 
Table 24 for a summary of the remaining correlations between affect and the ordinal severity of violent victimization items. 
Table 24

Correlations Between Severity of Violent Victimization Measured on an Ordinal Scale, and Affect

\begin{tabular}{|c|c|c|c|c|c|c|c|c|c|c|c|c|}
\hline \multirow{3}{*}{ PANAS-X Scales } & & & & \multicolumn{6}{|c|}{ Severity of Violent Victimization Items } & & & \\
\hline & \multicolumn{2}{|c|}{$\begin{array}{l}\text { Rating of } \\
\text { Distress }\end{array}$} & \multicolumn{2}{|c|}{$\begin{array}{l}\text { Length of Time } \\
\text { Bothered }\end{array}$} & \multicolumn{2}{|c|}{$\begin{array}{c}\text { Extent } \\
\text { Bothered }\end{array}$} & \multicolumn{2}{|c|}{$\begin{array}{l}\text { Rating of } \\
\text { Emotional } \\
\text { Severity }^{\mathrm{a}}\end{array}$} & \multicolumn{2}{|c|}{$\begin{array}{c}\text { Rating of } \\
\text { Psychological } \\
\text { Severity }^{\mathrm{a}}\end{array}$} & \multicolumn{2}{|c|}{$\begin{array}{l}\text { Rating of } \\
\text { Physical } \\
\text { Severity }^{\mathrm{a}}\end{array}$} \\
\hline & $r_{s}$ & $p$ & $r_{s}$ & $p$ & $r_{s}$ & $p$ & $r_{s}$ & $p$ & $r_{s}$ & $p$ & $r_{s}$ & $p$ \\
\hline General Negative Affect & .36 & .228 & -.01 & .970 & .49 & .093 & .29 & .333 & -.04 & .890 & .51 & .077 \\
\hline Fear & .41 & .167 & .09 & .781 & .42 & .149 & .21 & .496 & .16 & .592 & .46 & .115 \\
\hline Sadness & .16 & .610 & -.44 & .131 & -.17 & .582 & .13 & .680 & -.20 & .517 & .55 & $.050^{*}$ \\
\hline Guilt & .19 & .533 & -.04 & .885 & .14 & .644 & .21 & .483 & -.00 & .993 & .64 & $.019 * *$ \\
\hline Hostility & -.23 & .445 & .12 & .698 & .30 & .313 & .26 & .397 & .01 & .981 & .07 & .820 \\
\hline Shyness & -.57 & $.044^{*}$ & -.18 & .555 & .03 & .932 & .05 & .860 & .13 & .675 & .09 & .775 \\
\hline Fatigue & -.24 & .427 & -.59 & $.034 *$ & -.29 & .344 & .08 & .785 & -.39 & .193 & .51 & .078 \\
\hline General Positive Affect & .03 & .932 & .49 & .091 & -.03 & .933 & -.27 & .380 & .15 & .629 & -.31 & .296 \\
\hline Joviality & .16 & .613 & .62 & $.025^{*}$ & .31 & .308 & -.02 & .959 & .22 & .476 & -.45 & .124 \\
\hline Self-Assurance & .12 & 689 & .65 & $.016^{* *}$ & .18 & .568 & -.06 & .844 & .34 & .263 & -.13 & .671 \\
\hline Attentiveness & -.04 & .891 & .03 & .935 & -.58 & $.038^{*}$ & -.57 & $.044^{*}$ & -.44 & .133 & -.24 & .435 \\
\hline Serenity & -.72 & $.006 * *$ & .23 & .461 & -.27 & .376 & -.10 & .756 & -.15 & .633 & -.18 & .549 \\
\hline Surprise & -.14 & .658 & .32 & .286 & .25 & .406 & .04 & .896 & .34 & .250 & -.02 & .949 \\
\hline
\end{tabular}

Note. $N=13$. PANAS-X = Manual for the Positive and Negative Affect Schedule Expanded Form. $r_{\mathrm{s}}=$ Spearman's Rank-Order Correlation. Higher scores on the

PANAS-X are indicative of greater participant agreement on the items in that scale, whereas lower scores are indicative of less agreement. The "Rating of

Distress" variable ranged from $0=$ none, to $3=$ severe. The "Length of Time Bothered" variable ranged from $0=$ not at all, to $3=$ a month or more. The "Extent

Bothered" variable ranged from $0=$ not at all, to $4=$ very much.

a The variable ranged from $1=$ not severe, to $5=$ extremely severe. 
The final exploratory correlations conducted were between the ordinal severity of sexual victimization items and the affect scales. Not surprisingly, the more bothered individuals were by their sexual victimization, the more general negative affect they experienced, $r_{\mathrm{s}}(9)=.76, p=.007$. Individuals who were more bothered by their sexual victimization also tended to report higher levels of sadness $\left(r_{\mathrm{s}}[9]=.64, p=.033\right)$, fear $\left(r_{\mathrm{s}}\right.$ $[9]=.79, p=.004)$, and guilt $\left(r_{\mathrm{s}}[9]=.72, p=.012\right)$.

There was also a significant correlation observed between the Physical Severity item and the Fear scale, $r_{\mathrm{s}}(9)=.65, p=.030$. The correlation indicated that the more physically severe individuals perceived their sexual victimization to be, the higher their Fear score was in the PANAS-X. The other correlations were not significant at $p<.05$. See Table 25 for a complete outline of all the correlations between the ordinal severity of sexual victimization items and the affect scales. 
Table 25

Correlations Between Severity of Sexual Victimization Measured on an Ordinal Scale, and Affect

\begin{tabular}{|c|c|c|c|c|c|c|c|c|c|c|c|c|}
\hline \multirow{3}{*}{ PANAS-X Scales } & & & & \multicolumn{6}{|c|}{ Severity of Sexual Victimization Items } & & & \\
\hline & \multicolumn{2}{|c|}{$\begin{array}{l}\text { Rating of } \\
\text { Distress }\end{array}$} & \multicolumn{2}{|c|}{$\begin{array}{c}\text { Length of Time } \\
\text { Bothered }\end{array}$} & \multicolumn{2}{|c|}{$\begin{array}{c}\text { Extent } \\
\text { Bothered }\end{array}$} & \multicolumn{2}{|c|}{$\begin{array}{l}\text { Rating of } \\
\text { Emotional } \\
\text { Severity }^{\mathrm{a}}\end{array}$} & \multicolumn{2}{|c|}{$\begin{array}{c}\text { Rating of } \\
\text { Psychological } \\
\text { Severity }^{\mathrm{a}}\end{array}$} & \multicolumn{2}{|c|}{$\begin{array}{l}\text { Rating of } \\
\text { Physical } \\
\text { Severity }^{\mathrm{a}}\end{array}$} \\
\hline & $r_{s}$ & $p$ & $r_{s}$ & $p$ & $r_{s}$ & $p$ & $r_{s}$ & $p$ & $r_{s}$ & $p$ & $r_{s}$ & $p$ \\
\hline General Negative Affect & .38 & .254 & .12 & .735 & .76 & $.007 * *$ & -.01 & .977 & .24 & .469 & .52 & .099 \\
\hline Fear & .56 & .071 & .18 & .595 & .79 & $.004 * *$ & .24 & .469 & .46 & .153 & .65 & $.030 *$ \\
\hline Sadness & .30 & .371 & .16 & .633 & .64 & $.033 *$ & .01 & .983 & .12 & .727 & .54 & .086 \\
\hline Guilt & .30 & .371 & .48 & .139 & .72 & $.012 * *$ & .22 & .526 & .36 & .274 & .36 & .276 \\
\hline Hostility & .34 & .303 & -.41 & .208 & .07 & .843 & -.55 & .078 & .21 & .533 & .08 & .806 \\
\hline Shyness & .08 & .824 & -.02 & .965 & -.23 & .506 & -.25 & .450 & -.14 & .687 & -.16 & .633 \\
\hline Fatigue & -.26 & .436 & -.02 & .965 & .17 & .617 & .15 & .671 & -.06 & .859 & .14 & .681 \\
\hline General Positive Affect & .23 & .506 & .04 & .913 & -.44 & .171 & .35 & .290 & .18 & .605 & -.05 & .884 \\
\hline Joviality & .00 & - & -.37 & .270 & -.32 & .330 & .16 & .631 & -.28 & .401 & -.18 & .607 \\
\hline Self-Assurance & .49 & .129 & .17 & .617 & -.24 & .470 & .38 & .251 & .29 & .383 & .23 & .505 \\
\hline Attentiveness & .19 & .573 & .19 & .572 & .08 & .808 & .62 & .044 & .20 & .559 & .41 & .215 \\
\hline Serenity & .23 & .502 & -.08 & .819 & -.58 & .060 & .08 & .819 & -.06 & .858 & -.03 & .935 \\
\hline Surprise & .35 & .286 & .14 & .672 & -.02 & .964 & .18 & .597 & .06 & .869 & -.08 & .824 \\
\hline
\end{tabular}

Note. $N=11 .{ }^{*} p<.05 .{ }^{* *} p \leq .01$. PANAS-X = Manual for the Positive and Negative Affect Schedule Expanded Form. $r_{\mathrm{s}}=$ Spearman's Rank-Order Correlation Higher scores on the PANAS-X are indicative of greater participant agreement on the items in that scale, whereas lower scores are indicative of less agreement. The "Rating of Distress" variable ranged from $0=$ none, to $3=$ severe. The "Length of Time Bothered" variable ranged from $0=$ not at all, to $3=\mathrm{a}$ month or more. The "Extent Bothered" variable ranged from $0=$ not at all, to $4=$ very much.

${ }^{a}$ The variable ranged from $1=$ not severe, to $5=$ extremely severe. 


\section{Discussion}

\section{Gait Pattern Vulnerability}

Study 1 consisted of seven major hypotheses: The first hypothesis predicted that individuals with prior victimization experiences would exhibit vulnerable gait cues. Two independent raters who were blind to the victimization history of the walkers coded each participants' gait. Five movement categories were used to code the walkers: 1) stride length, 2) type of weight shift, 3) body movement, 4) type of walk, and 5) foot movement (Grayson \& Stein, 1981). A “typical” victim, according to Grayson and Stein’s (1981) movement categories, would exhibit a long or short stride (not a medium stride), shift their body weight laterally, diagonally, or with an up/down movement (not threedimensionally), walk gesturally (not posturally), move unilaterally (i.e., one side at a time) as opposed to contralaterally, and lift their feet (not swing them; see Appendix A).

As expected, individuals with a victimization history exhibited a significantly different gait pattern relative to the control group (the non-victimized group). More specifically, individuals with a victimization history (sexual and/or violent) displayed more vulnerable movement cues than did individuals without a victimization history. This finding is consistent with previous literature (e.g., Book et al., 2013; Grayson \& Stein, 1981; Wheeler et al., 2009), and suggests that gait may be a valid indicator of vulnerability. Importantly, the current study removed extraneous cues such as clothing, age, and attractiveness by filming the targets in point-light conditions. Therefore, the coding of each walker's gait was based exclusively on their movement pattern. The results thus provide convincing evidence that individuals who have been victimized produce a considerably different movement pattern compared to those who have not experienced victimization. 
While it appears that gait may have an impact on one's vulnerability to victimization, and play a role in the likelihood of one experiencing victim recidivism, it is not possible to determine causation from the present findings. Therefore, it remains unclear whether vulnerable gait characteristics were present in individuals before the victimization event, or if they occurred as a result of the victimization event. Given the nature of the research, it is unlikely that even longitudinal studies could explore the phenomenon. However, future research exploring the physical (i.e., non-verbal) aftereffects experienced by individuals who have been victimized may provide some understanding as to the direction of the relationship.

\section{The Relationship between Victimization History, Gait, and Personality}

The second, third and fourth hypotheses in Study 1 pertained to the personality of the walkers. Given the underlying link between vulnerability and submissiveness (e.g., submissiveness can be defined as the tendency to behave in helpless, appeasing, and fearful ways; Carli et al., 1995), the existing literature has largely assumed that the vulnerable gait pattern is an expression of submissiveness. To explore this issue, analyses were conducted to explore the relationship between personality, victimization history, and gait pattern. It was predicted that individuals who had been victimized and exhibited vulnerable gait features would be more likely to report submissive personality traits, and that personality would (at least partially) explain the relationship between victimization history and gait.

The hypotheses were not supported; the relationship observed between submissiveness and victimization history was not significant, nor was there a significant association between submissiveness and gait. However, contrary to expectation, the 
results indicated that individuals who exhibited the "victim walk" pattern were significantly more likely to report dominant personality characteristics. In other words, individuals with vulnerable features present in their gait were significantly more likely to self-report personality characteristics consistent with Assured-Dominance. A mediated regression analysis was then conducted to examine whether or not dominance could account for the relationship between victimization history and gait. The results suggested that both previous victimization and dominance could independently predict the vulnerability level of one's gait. However, dominance could not explain the association between victimization history and gait.

The relationship between victimization history, personality, and gait remains unclear. The results suggest that dominant individuals and those who had been victimized are more likely to display a vulnerable gait pattern. However, dominant individuals in the present sample were no more likely (than anyone else) to have a victimization history (i.e., there was no relationship between any of the personality traits and reported victimization history). It is possible that dominant individuals exhibit a noticeably different (though not necessarily "vulnerable") gait pattern. Perhaps dominant individuals in the present sample were scored as having gait features that were vulnerable, when in reality they simply exhibited a more salient walking pattern and/or produced exaggerated movements. Indeed, when Grayson and Stein (1981) asked their sample of offenders why they chose certain walkers, many noted that they selected those who "...looked different" (p. 74).

Evolutionary theory would suggest that signals of dominance, which are performed by affluent members of non-human primate groups, have likely been retained 
(to some degree) in humans. Indeed, research suggests that high-status non-human primates display specific movement patterns in order to inform rivals and/or potential mates of their status (e.g., Mazur, 1985). Similarly, research examining the cueing of dominance in humans suggests that dominant individuals display noticeably different movement cues compared to others (e.g., increased eye contact; Richards et al., 1991; Simpson et al., 1993). However, the adaptive quality of dominant movement cues would not be particularly useful if research indicates that such cues are similar to those which connote vulnerability. Future research may thus wish to explore whether dominant and vulnerable gait patterns are exceedingly similar.

It is also possible that the walkers who exhibited vulnerable gait cues simply overcompensated in their personality self-reports and scored themselves as more dominant than they truly are. On the other hand, the sample itself may have been biased; participants were those who were willing to participate in a filmed movement study, wherein they were asked to wear a tight-fitting Velcro suit and report on their victimization history. Extremely submissive individuals may have been less inclined to participate in a laboratory study (when they have the option to participate in online studies) and particularly so when the research involves recording them on a set and asking them about their victimization history. The research itself may thus have unintentionally recruited outgoing, dominant participants (at least to some extent). A sample consisting of more extreme submissive individuals may have produced different results. Nevertheless, it appears that vulnerable gait features are expressed in selfreported dominant individuals. Additional research is necessary to understand how dominance, gait vulnerability, and prior victimization interrelate. 
A recent study by Sakaguchi and Hasegawa (2006) questioned the recorded walkers about their personality and victimization history. They found that the walkers who were rated as likely victims by a separate sample of observers self-reported themselves to be neurotic, shy, low in self-monitoring, and introverted (i.e., correlates of submissiveness). The observers thus chose walkers who were submissive as those most vulnerable to future victimization. Interestingly, however, the observers were not accurate in their ratings (i.e., the walkers who were submissive, which the observers chose as victims, did not actually have a victimization history). Consistent with Sakaguchi and Hasegawa (2006), the present results revealed little to no association between victimization history and submissiveness.

It appears that while much of the current literature (and the general population) assumes that individuals who are submissive are at an increased risk of experiencing victimization, this may not be the case. It is possible that individuals who are submissive may actually design their lives in a way that lessens their chances of experiencing victimization. For example, Beauregard et al. (2007) outline how an individual's recreational/routine habits, occupational choices, and even transportation patterns can impact an offender's choice of victim. Submissive individuals may be less inclined to drink alcohol or party on a regular basis, and they may choose professions that are generally considered safe and/or those which do not put them in regular contact with the public. Therefore, while submissive individuals may be "easy victims" to exploit, offenders may actually have fewer opportunities to target them (i.e., they may not be opportune victims because they are less likely to engage in "risky" routine activities). The availability and/or access to "easy (i.e., submissive) victims" may need to be examined. 
Replication with a larger sample of walkers is needed in order to ensure the reliability of the results (i.e., a larger sample of walkers may lend credence to the present findings, particularly the regression analyses, wherein 40-50 participants per predictor are often recommended; Cohen \& Cohen, 1983; Tabachnick \& Fidell, 2013). A number of factors affected the sample size of the walkers in the current study: Limited time (participants were remunerated with a bonus percentage in one of their Psychology classes; thus, Study 1 was restricted to occur within the active SONA period), resources (access to the Motion Capture Laboratory was limited and required advanced booking; an available technician who could operate the point-light system during each session was also necessary), and participants (the study required a sample of participants willing to participate in a laboratory study, who had a history of victimization) resulted in a small sample of walkers. Given the relatively small sample of walkers used in the current study, the results must be interpreted cautiously.

The fidelity of the surveys that were completed by the walkers must also be considered. The surveys present each participant's perception of them self, and fail to incorporate the views of others. Therefore, it may be argued that the survey responses contain some degree of bias. Indeed, in regards to the personality survey (i.e., the IASRB5) some research suggests that individuals perceive themselves overly positively, or as the type of person they would like to be, as opposed to the type of person that they actually are (e.g., Kruger, 1999). Similarly, considering that the victimization survey was completed from one's own perspective it may represent a partial/one-sided account of the victimization incident; certain details may have been emphasized or omitted. Future research is encouraged to verify the walker's traits, states and history of victimization 
using corroborated data (e.g., by obtaining additional, secondary reports from close sources).

In addition, although participants were asked to complete the survey honestly, self-reports of personality (and affect) are limited in that the constructs examined can fluctuate and adapt. For example, personality may change as a result of an individual's circumstances and relationships, or it can simply evolve over time (e.g., Hampson \& Goldberg, 2006). Therefore, the reports provided by participants are only true to the extent that they reflect their present emotional state and personality traits. Thus, it is possible that an individual may experience a shift in their personality or affect following a significant life event; if gait is tied to one's personality, then this relationship may change accordingly.

That being said, research suggests that personality, though developing and in a state of change in one's early years, tends to solidify by adulthood (e.g., Hampson \& Goldberg, 2006). For example, a 40-year longitudinal study conducted by Hampson and Goldberg (2006) on 799 males and females suggested that Big Five personality characteristics had fairly low test re-test reliabilities in childhood (.22 to .53). However, in adulthood, ${ }^{21}$ personality stability peaked (.70 to .79$)$. Therefore, although caution must be exercised when generalizing the findings from the current study (given the limitations discussed above), it is unlikely that major differences in personality (and/or affect) would be observed in the sample if they were tested weeks or months later.

\section{The Relationship between Victimization History, Gait, and Affect}

\footnotetext{
${ }^{21}$ The nearly 800 male and female participants included in Hampson and Goldberg's (2006) study were tested as elementary children and 40 years later when they were in mid-life. Given that personality may still be fluctuating in one's early adulthood (e.g., 18-25), and the majority of the walkers in the current sample were young adults, their personality may still be in a state of change. However, it is unlikely to be fluctuating to the same extent that it does in childhood.
} 
The fifth, sixth and seventh hypotheses in Study 1 aimed to investigate the affect of the walkers. Recent research (e.g., Schneider et al., 2014) has provided evidence demonstrating that internal emotional states appear to be expressed in movement. Given the effects on post-assault victims, a significant relationship was expected between negative affect, prior victimization experiences, and gait movement. In addition, it was hypothesized that affect would (at least partially) explain the vulnerable gait pattern observed in previously victimized individuals.

Contrary to expectation, in the present sample, the walkers' self-reported affective state(s) did not demonstrate any relationship with their movement cues. The emotional state of the walkers was also not related to their victimization history. Therefore, in general, prior victimization experiences did not appear to relate to the emotional wellbeing of the individuals in the current sample.

Differences in coding may explain why previous literature has found a relationship between affect and movement cues, while the current study did not. For example, the current study coded the walkers' gait movement according to Grayson and Stein's (1981) criteria, and subsequently correlated these gait ratings with the walkers' self-reported affect. Schneider et al. (2014), on the other hand, did not code and differentiate the movement patterns according to the same criteria, nor did they correlate gait vulnerability with the walkers' self-reported affect. Instead, they outlined how emotional displays become expressed in movement, and then used FNIRS to demonstrate how negative emotions are detected more easily than positive emotions, by observers.

It is possible that while affect may be expressed in movement, it is not associated with vulnerable gait movement in particular. In other words, negative affective states 
such as sadness and/or hostility do not seem to produce the same movement features that comprise the "victim walk" (i.e., negative affect does not appear to be expressed with unilateral movement, lifted feet, long or short strides, etc. [characteristics associated with the "victim walk"]). However, given that the participants in the current study were aware that they were being recorded, they may have been less likely to exhibit their true emotional states. ${ }^{22}$ In Wheeler et al.'s (2009) study, for example, the walkers were filmed covertly and thus their most natural gait pattern was captured.

Although affect is argued to be relatively stable (e.g., Watson et al., 1998; Watson $\&$ Clark, 1994), changes in mood may occur as a result of the situation or interaction one finds them self in; it is unlikely that participants would express the same movement patterns in a laboratory study that they would if they were alone or not aware that they were being watched. Given that the participants in the current study were asked to focus on a specific (memory) task and were clearly aware that they were being filmed, it is possible that an expression of their genuine emotion was not captured.

Furthermore, because participants were not asked to focus on any specific emotion, it is possible that the walkers affect was not extreme enough for differences to be detected. In Schneider et al.'s (2014) study, the walkers were explicitly asked to focus on a life event that made them either happy or sad. Future research may wish to record individuals walking in stereotypically sad, angry, and/or happy styles, and test whether vulnerable gait cues are associated with these more intense affective displays. In addition,

\footnotetext{
${ }^{22}$ Point-light display is limited in that it is very difficult to deceive individuals into believing that they are not being recorded. The system requires that one wear a fitted black suit with light-reflective markers attached to it. There are also upwards of 10 cameras in the Motion Capture Laboratory, which produce red light during the recording process. Therefore, while it would be ideal to capture an individual's natural movement (i.e., when they are unaware that their movement is being recorded), there were both ethical (given the vulnerable sample of participants), and practical (given the point-light display) restrictions that prevented the covert recording of the walkers.
} 
research investigating differences in gait across samples of clinically depressed individuals, and/or those experiencing emotional extremes, would provide crucial information regarding the impact of affect on non-verbal behaviour.

It is important to note that Study 1 utilized a convenience sample. Although the student sample met the needs of the study (i.e., they reported victimization histories, and could report on a variety of personality characteristics and affective states), a community sample would enable greater generalizability of the results. Some research suggests that student populations differ in important ways from the general public. For example, college students appear to be at a higher risk to experience victimization (e.g., Breitenbecher, 2000; Fisher, Sloan, Cullen, \& Lu, 1998), and are particularly more likely than the general public to experience sexual assault (e.g., Abbey, 2002; Abbey \& McAuslan, 2004; Sable, Danis, Mauzy, \& Gallagher, 2006). This increased vulnerability to victimization may have implications in terms of measuring gait cues (e.g., perhaps more college students will exhibit a vulnerable gait relative to non-college students given that more of them will have been victimized).

\section{The Effect of Time, Frequency, Severity, and Type of Victimization, on Gait}

The final research question in Study 1 pertained to the specific victimization histories of the walkers. The walkers were asked to categorize their victimization as either violent or sexual, and to report on the time frame of the event (i.e., when it occurred), the frequency with which they had experienced each type of victimization, and the subjective severity of the experience. As there has been no known investigation examining the effect of specific victimization experiences (e.g., singular versus multiple victimization) on gait vulnerability, no formal hypotheses were made. However, more 
frequent, as well as more recent and severe victimization experiences, were assumed to have a greater impact on movement cues.

The exploratory analyses suggested that walkers who had been violently victimized were significantly more likely to have a vulnerable gait, while those who experienced sexual victimization were less likely. There are a number of differences between violent and sexual victimization that may explain their differential relationship with gait. Violence, by its very definition, is aggressive, forceful, and hostile. Thus, it may be argued that victims of violence are at a greater risk for experiencing physical harm, and that this in turn may affect their gait movement (i.e., physical injury resultant from a violent victimization event may actually alter gait). Indeed, the current results suggest that more individuals who were violently victimized reported that their victimization was physically severe (i.e., nearly half of all individuals who reported that they were violently victimized rated the physical severity of their experience between 3 and 5 on the 5-point scale), relative to those who had been sexually victimized.

On the other hand, individuals who experienced violent victimization also tended to, on average, perceive their victimization as more psychologically and/or emotionally severe, relative to those with a history of sexual victimization (e.g., they were more apt to experience feelings of danger at the time when they were violently experienced, more individuals reported that they were bothered to a greater extent by the event and for a greater period of time, etc.). Therefore, it is also possible that the impact of violent victimization at the psychological level, may have an effect on the way that one walks. Nevertheless, it must be reiterated that the direction of the relationship is unknown (i.e., while it is possible that experiencing violent victimization altered one's gait movement, 
vulnerable gait features may also have existed prior to one experiencing victimization), and causality cannot be presumed (i.e., one cannot assume that experiencing violent victimization causes a change in one's movement pattern).

Interestingly, significant relationships were also observed between violent victimization and the personality trait of dominance, as well as between violent victimization and openness to experience. Although prior analyses indicated that dominance could not account for the relationship between gait and victimization history, it is possible that dominance could explain the relationship between gait and violent victimization specifically. It is also possible that a combination of personality traits (e.g., dominance and openness to experience) could account for the relationship between prior victimization experiences and vulnerable gait cues. Unfortunately, the small sample size in the current study restricted the number of tests that could be conducted so these possibilities could not be explored empirically. Furthermore, given that victimization history was a nominal variable in the present analyses it could not be used as a mediator. Future analyses, which designate gait as the outcome, personality as the predictor, and victimization history as the mediator, is encouraged. It is possible that even a stepwise regression model inclusive of all possible personality characteristics could provide additional information regarding the predictive ability of personality on violent (or sexual) victimization in particular. It may also be of interest to regress gait on victimization history.

The majority of the correlations between affect and type of victimization were not significant. However, unsurprisingly, there was a significant, positive relationship observed between violent victimization and attentiveness (or alertness). Regardless of 
whether individuals with violent victimization histories experienced a surprise attack by a stranger, or prolonged abuse by a known other (e.g., domestic abuse), it is reasonable that they would employ increased vigilance. Future research may want to consider asking victimized walkers who perpetrated the offence against them (i.e., known or stranger) in order to confirm this presumption.

As previously noted, the current sample was too small to isolate individuals who had been victimized both sexually and violently. Several participants who were victims of violent crime had also been sexually victimized (and vice versa). The findings that differ by "type of victimization" (i.e., sexual versus violent) must therefore be interpreted cautiously. A larger sample that allows for a separate analysis examining those who have experienced multiple types of victimization is necessary and should be a focus of future research.

The time frame of the walkers' victimization experiences and the frequency with which they reported having been a victim of crime, appeared to have little effect on their gait. ${ }^{23}$ However, various correlations between time frame, frequency, personality, and affect were significant. For example, individuals who have been sexually victimized multiple times reported higher levels of guilt, as well as fear. As individuals who have experienced victim recidivism often attribute blame to themselves (e.g., Filipas \& Ullman, 2006), these results are not surprising. There was also a significant relationship observed between the time frame of one's violent victimization and warm-agreeableness, as well as between the time frame of violent victimization and cold-heartedness. Individuals who were violently victimized long ago (i.e., further back in their past) were

\footnotetext{
${ }^{23}$ In addition to the correlations described in the above results section, a series of ordinal regressions were conducted to investigate the effect of victimization type, time frame, frequency, and severity, on gait. However, the items had no significant effect on the vulnerability level of one's gait pattern.
} 
significantly more likely to report personality traits consistent with cold-heartedness, and significantly less likely to report warm-agreeable type traits. Again, these results are unsurprising.

Interestingly, cold-heartedness persisted as a significant item across various correlations. For example, those who scored high on cold-heartedness perceived their violent victimization experience to be less psychologically severe relative to others, and reported that they did not feel as though they were in danger at the time when they were sexually victimized. Early traumatic events may become redefined by individuals (e.g., as a coping strategy), and interpreted as less severe and/or dangerous than they truly were. Given the after-effects of victimization, it is also possible that early victimization experiences impact the personality of some individuals, perhaps altering individuals to behave ruthlessly or cruel. To date, research has mainly examined the levels of fear, depression, and anxiety experienced by victims of crime (e.g., Norris \& Kaniasty, 1994). However, Norris and Kaniasty (1994) also refer to the hostility exhibited by individuals who have been victimized (e.g., perhaps employed as a defence mechanism), as well as the avoidance tactics that they engage in. However, as causality is undetermined at this point, it remains unclear whether cold-hearted traits were present prior to the victimization event, or if they occurred as a result of the experience.

Lastly, the subjective severity of the walkers' victimization history was analyzed. As noted above, individuals who experienced violent victimization tended to perceive the event as more severe (on average, across multiple items), relative to those who had been sexually victimized, and violent victimization was significantly related to gait (i.e., individuals who experienced violent victimization tended to exhibit more vulnerable gait 
features). However, there was a significant relationship observed between participants' feeling of danger at the time when they were sexually victimized, and their gait, as well as between participants' feeling of distress during their sexual victimization, and their gait. Those who perceived themselves to be in danger, and who experienced higher levels of distress at the time when they were sexually victimized, were more likely to exhibit a vulnerable gait pattern. This may indicate that more severe and/or very serious (defined subjectively) sexual victimization experiences may also impact the vulnerability of one's walk.

It is possible that a more exhaustive and/or psychometrically established measure of severity would have produced different results. Given the study's time restrictions, only certain scale items from the Brief Trauma Questionnaire (Schnurr et al., 2002) and the Trauma History Screen (Carlson et al., 2011) were adopted into the Victimization History Questionnaire Extended. Nevertheless, it appears that severity may have an effect on gait vulnerability. As it is not possible to know the direction and/or causal relationship between severity and gait, it is unclear whether or not physical (or emotional) injury resulting from a severe victimization event caused the vulnerable gait, or if the gait pattern was present before the event and simply increased the individual's vulnerability to experience victimization. Future research may wish to consider investigating the effect of coping on gait movement cues. If vulnerable gait cues arise following a victimization experience, it is possible that individuals who engage in better coping strategies and/or who display healthier coping mechanisms post-attack will exhibit less vulnerable movement cues. 
Interestingly, individuals who were more self-assured, less introverted, less submissive and less shy (i.e., taken together, these characteristics seem to suggest individuals that are potentially more dominant) appeared to experience more severe victimization experiences. For example, individuals who reported that they were selfassured were more likely to experience serious injury as a result of their victimization, and to report that they were bothered for a greater period of time. On the other hand, individuals who were submissive, introverted, and shy were significantly less likely to report distress during their victimization. Thus, it is possible that individuals who are more dominant tend to experience more severe victimization experiences. Recall that higher levels of reported dominance significantly predicted greater gait vulnerability. Overall then, it appears possible that individuals who are dominant, and who have experienced severe victimization, are those most likely to exhibit the vulnerable gait pattern.

Again, it is unclear if individuals were dominant prior to, or after, the victimization event, and/or whether or not they experienced the victimization event prior to, or following, exhibition of the vulnerable gait pattern. However, the results did suggest that the more time that passed since one's (sexual) victimization experience, the more dominant individuals reported themselves as being. Perhaps individuals who have experienced severe victimization attempt to overcompensate for their underlying feelings (i.e., their vulnerability) by perceiving themselves to be more dominant than they truly are. However, consistent with the KSD principle, the body movement of dominant individuals appears to remain vulnerable; essentially, the sadness, guilt, and distress 
experienced by individuals who have been severely victimized, "leaks" into one's gait, unintentionally disclosing authentic internal information.

\section{Study 2: The "Observers"}

Study 2 utilized the recorded point-light displays to investigate the extent to which gait is a reliable indicator of victim vulnerability ratings. Kinematic point-light display technology has rarely been used to investigate gait as a victim selection cue. Gunns et al. (2002) demonstrated that vulnerability ratings continue to correspond to the prototypical "victim walk” pattern (Grayson \& Stein, 1981), even when gait cues are isolated through the use of point-light displays. However, Sakaguchi and Hasegawa's (2006) results indicated rater consensus, but not accuracy. Few studies have used pointlight display to examine observer ratings of victim vulnerability in conjunction with the self-reported victimization history of the walkers. Therefore, Study 2 aimed to confirm that individuals agree within one another on which target(s) will be chosen for future victimization, and that their ratings are accurate to the extent that those chosen as future victims do in fact self-report a victimization history.

In an effort to disentangle the reasons why previously victimized individuals walk differently relative to others, Study 1 examined walker personality and affect. However, it remains unclear if observers can accurately detect affect and/or personality from gait, and if either can be used to help make decisions about an individual's vulnerability. Some research has suggested that the vulnerable gait pattern detected by observers may be the product of the walker's submissive personality (e.g., Myers et al., 1984). However, another body of literature has provided evidence outlining both the translation of affective states into gait, as well as the accuracy of zero-acquaintance observers in 
detecting affect from gait (e.g., Ikeda \& Watanabe, 2009; Schneider et al., 2014). Therefore, Study 2 performed an exploratory analysis examining observer perceptions of walker personality and affect. It is the first known study to examine the detection of personality traits versus affect, from gait cues, in a sample of self-reported victims.

Much of the current research has defined victimization as being equal to or greater than bullying (Book et al., 2013; Ritchie, 2014). However, the impact of specific types of victimization (i.e., sexual, violent) on gait cues and observer accuracy has not been thoroughly explored. In other words, it is possible that individuals are more adept at identifying certain types of victimization from body movement cues, relative to others. Therefore, Study 2 aimed to more thoroughly examine differences in rating accuracy across sexual and violent victimization.

Consensus. Some research argues that a distinction between consensus and selfother agreement is necessary in order to have a thorough understanding of the detection ability of observers (Yeagley et al., 2007). Therefore, the current study aimed to first explore the consensus (i.e., agreement) among observers regarding which point-light display walkers were most likely to be chosen as a future victim.

$\boldsymbol{H}_{1 .}$ It was expected that, in line with previous research, observers would agree on which walker(s) were most likely to be victimized. It was further hypothesized that observers would agree on which individuals were submissive and which exhibited high negative affect.

Accuracy. Prior research has indicated that observers appear to select past victims as future victims based on "thin-slices" of gait information (e.g., Wheeler et al., 2009). Therefore, self-other agreement accuracy was present if the walkers who indicated that 
they had been a previous victim, and exhibited vulnerable gait cues, were chosen for future victimization by observers. Similarly, observer accuracy for personality and affect was determined if the observers' ratings of the walkers' personality traits and affective states were strongly positively associated with the walkers' own self-reports of their personality and affect.

$\boldsymbol{H}_{2}$. It was expected that individuals who exhibited the characteristic "victim walk" pattern and had a self-reported history of victimization, would be identified as those most likely to be victimized in the future.

$\boldsymbol{H}_{3 .}$ Consistent with prior research suggesting that observers may be unaware of the cues used when making decisions regarding vulnerability (e.g., Grayson \& Stein, 1981), observer ratings were not predicted to necessarily correlate with the walkers' selfreported personality and affect. In other words, although observers may agree on which targets are submissive and exhibiting negative affect, the extent to which these ratings are accurate may vary.

The effect of the observers' perceptions of the walkers' personality and affect on ratings of vulnerability. It remains unclear whether or not zero-acquaintance observers rely on their perceptions of others' personality and affect when they make decisions regarding vulnerability. For example, if an individual is perceived to be sad, does their apparent sadness have an effect on the vulnerability rating provided for that individual? The present study explored the extent to which the observers' perceptions of the walkers' personality and affect predicted their vulnerability to victimization.

$\boldsymbol{H}_{4 .}$ Based on previous research (e.g., Sakaguchi \& Hasegawa, 2006), it was expected that an observer's perception of the personality and/or affect of a walker would 
dictate the vulnerability rating bestowed. More specifically, it was expected that perceptions of walker submissiveness and/or negative affect, would result in higher ratings of vulnerability (i.e., walkers that appeared to be submissive or experiencing negative affect, would be rated as more vulnerable to victimization).

\section{The effect of the walkers' own (self-reported) personality and affect on}

likelihood to experience victimization. Since individuals may perceive their own personality and/or affect differently from how others do, an exploratory analysis was also conducted to examine whether the walkers' own self-reported personality traits and affective states could predict their likelihood of having experienced violent and/or sexual victimization. Because it was unclear if observer perceptions would be similar to the true (self-reported) personality and affect of the walkers (i.e., the way that a stranger-observer perceives a walker may not be how the walker self-interprets themselves), no formal hypotheses were made.

\section{Method}

\section{Participants}

The sample for Study 2 consisted of 129 community members recruited via the online survey tool, Qualtrics. ${ }^{24,25}$ Each survey took approximately 30 minutes to complete and participants received between $\$ 0.80$ and $\$ 1.20$ (depending on where Qualtrics recruited them from) as compensation for their participation. Approximately $55.3 \%(n=$ 52) of the sample was female and $44.7 \%(n=42)$ was male. Participants ranged in age

\footnotetext{
${ }^{24}$ Qualtrics is an online survey platform (Qualtrics, 2009). It can be used to build surveys/questionnaires which can then be released to students (e.g., through SONA). Or, alternatively, individuals across the country can sign-up to take part in online surveys which are hosted by Qualtrics, in return for a small amount of money.

${ }^{25}$ An apriori power analysis was conducted using $\mathrm{G}^{*}$ Power (Faul et al., 2009). A two-tailed, correlational (Point-biserial) model, with an effect size set at .3 (medium; Cohen, 1992), and 95\% power, estimated that a sample size of 134 would be required. Thus, the sample that was obtained retained power.
} 
from 24 to 81 years $(M=49.14, S D=13.93) .{ }^{26}$ There were no significant differences in age between males $(M=46.83, S D=12.73)$, and females $(M=46.31, S D=13.99), t$ $(92)=-.19, p=.851$. The majority of participants $(90.6 \%, n=116)$ were Caucasian; approximately $5.5 \%(n=7)$ were Asian, one individual $(.8 \%)$ was African Canadian/Black, one individual (.8\%) was First Nations/Aboriginal Canadian/Native Canadian, and 2.3\% $(n=3)$ designated themselves as from another unspecified ethnic background. There was no significant difference observed between the number of Caucasian males who participated $(45.2 \%, n=38, z=.1)$ and the number of Caucasian females who participated $(54.8 \%, n=46, z=-.1), \chi^{2}(4)=2.39, p=.664 .^{27}$

\section{Measures}

Participants were required to complete a two-part online questionnaire. Part 1 consisted of the same demographic questionnaire used in Study 1 (see Appendix C). It was comprised of three questions that asked the participant to indicate their age, gender, and racial/ethnic background. Part 2 consisted of a 28-item Victim Vulnerability Rating Questionnaire (see Appendix L).

Victim Vulnerability Rating Questionnaire. The Victim Vulnerability Rating Questionnaire is a measure designed to assess participant perceptions of target vulnerability. It asks participants to rate walker vulnerability on a 10-point rating scale (1 $=$ not at all vulnerable, $10=$ completely vulnerable). Initially, Grayson and Stein (1981) developed a 10-point "scale for rating assault potential" using a sample of incarcerated offenders. The scale consisted of terminology used by the offenders (e.g., $1=$ A very easy rip-off, $10=$ Would avoid it, too big a situation. Too heavy). The scale was later adapted

\footnotetext{
${ }^{26}$ The study was restricted to Canadian citizens that were 18 years of age or older.

${ }^{27}$ All other categories of ethnicity had cell counts of less than 5; therefore, chi-square was not conducted on these items.
} 
by Wheeler et al. (2009) who sampled an undergraduate population. The students were asked to place themselves in the role of a mugger and indicate how vulnerable each target was to victimization using a 10 -point scale $(1=$ not at all vulnerable to victimization, 10 = completely vulnerable to victimization). Book et al. (2013) used the same rating scale as Wheeler et al. (2009) with psychopathic offenders and defined victimization as "assault with the intent to rob or steal from the victim." An expanded version of the questionnaire was recently used in an unpublished undergraduate honors thesis (Ritchie, 2014), which sampled students at Carleton University; this expanded questionnaire (with slight modifications, as detailed below) was used in the current study.

The first set of questions in the expanded version developed by Ritchie (2014) asks participants to rate each walker's vulnerability on the 10-point rating scale previously described $(1=$ not at all vulnerable, $10=$ completely vulnerable $)$ to sexual, violent, and any victimization. Ritchie's expanded questionnaire defines sexual victimization for participants as "either sexual abuse and/or sexual assault and any attempted assaults" and violent victimization as "all sexual victimization noted above in addition to bullying, and any of the following crimes: robbery, threats, physical abuse, sexual assault, sexual abuse, and stalking” (p. 28).

The current research aimed to obtain a more detailed understanding of observer detection accuracy. Therefore, the definitions for sexual and violent victimization, were revised to more clearly demarcate them. The revised definition for sexual victimization was, "sexual abuse, any non-consensual sexual activity (e.g., sexual assault, rape, etc.), threat of unwanted sexual contact, sexual trafficking, sexual defamation (e.g., sending of nude photos), sexual coercion, etc." The revised violent victimization definition was, 
"physical bullying, in addition to any of the following crimes: Robbery, mugging, assault, physical abuse, neglect, harassment, battery (e.g., intimate partner violence), kidnapping, gang violence, threats, etc.”

The modified definitions mapped more precisely on to how the walkers were asked about their own victimization history in Study 1. It was the hope of the researcher that by establishing greater separation between the types of victimization, and creating parallel definitions (between how the walker's define the different types of victimization, and how the observers rate their victimization), a clearer estimation of self-other agreement accuracy could be achieved.

Ritchie's (2014) questionnaire also consists of three items which ask participants to state whether the subject is vulnerable to sexual, violent, and any victimization using a yes/no format as opposed to the rating scale format. Next, a set of seven questions asks about observer perceptions of the target's independence, exploitability, and capability, among others. Participants are asked to judge statements such as "this person is strong" on a scale ranging from 1 (not at all true) to 10 (completely true). These questions relate to observer perceptions of vulnerability and help provide context for the ratings.

For the purposes of the current study, an additional set of twelve statements were added to the questionnaire in order to obtain an indication of the observers' perceptions of the walkers' personality and affect; four relate to the perceived personality of the target walker, and eight relate to the perceived affect of the target walker. For example, a statement related to the perceived affect reads, "this person is sad." The final set of three questions in the original questionnaire (Ritchie, 2014) asks participants to indicate whether they believe the target actually has been a victim (of sexual, violent, and any 
type of crime) in the past using a yes/no answer format. These additional questions were left in for descriptive purposes.

\section{Procedure}

Individuals who met the criteria to participate (i.e., they were Canadian citizens, who were 18 years of age or older, and could understand English), were recruited by a Qualtrics panel. Those who were interested in participating could click on a link provided by Qualtrics, and the link directed them to a consent form (see Appendix M). If they chose to consent, the system then redirected them to the study (which was also hosted by Qualtrics).

The online survey began with a set of instructions (see Appendix N). The instructions informed participants that following a short demographic questionnaire, 14 videos $^{28}$ of individual's walking would be shown. Although the videos were randomized in an attempt to control for order effects, the number of times that each video was shown across participants was controlled to ensure that each video was seen by approximately the same number of observers. Therefore, genuine (complete) randomization was not met. Consistent with Book et al. (2013), Ritchie (2014), and Wheeler et al. (2009), the videos lasted approximately 10 seconds each and the targets were shown walking away from the participants (i.e., with their backs to the camera). After each video, the Victim Vulnerability Rating Questionnaire appeared and asked participants to rate the last seen walker on their vulnerability to sexual, violent, and general (any) victimization.

\footnotetext{
${ }^{28}$ Although 28 videos were recorded altogether, each participant in Study 2 viewed only a randomly selected half of the videos; this was done in an attempt to reduce participant fatigue and maintain internal validity. Each video was approximately 10 seconds long, and each corresponding Victim Vulnerability Rating Questionnaire took participants an average of 2.5 minutes to complete. It took participants, on average, 35 minutes to complete the entire study.
} 
In accordance with Wheeler et al.'s (2009) study, participants were not made aware of any vulnerability cues prior to rating, and were provided as much time as necessary to provide their ratings before moving on to the next video. When they were ready to observe the next video they simply selected the "Next" button at the bottom of the webpage. If the participant chose to withdraw at any point during the study, they were compensated and redirected to the debriefing form (see Appendix O). Upon completion (or withdrawal), all participants viewed the debriefing page. The entire Study 2 package was approved by the Carleton Ethics Committee for Psychological Research (REB \#15057).

\section{Results}

\section{Preliminary Data Screening}

Missing data. Each participant in Study 2 viewed half of the walker videos (i.e., the 28 videos were randomized, and each participant observed 14 of them). They then completed the Victim Vulnerability Rating Questionnaire following each video clip (i.e., they were asked to complete the same questionnaire 14 times). Between 58 and 66 participants viewed each video and completed each accompanying questionnaire. For example, 65 randomly-selected individuals viewed the video of Walker \#001 and subsequently completed the Victim Vulnerability Rating Questionnaire, 64 individuals viewed Walker \#006 and completed the accompanying questionnaire, 58 individuals viewed Walker \#011 and completed the questionnaire, etc. In order to examine the missing data, the file was filtered by video and a missing value analysis was conducted on the questionnaire items pertaining to each specific video. The amount of missing data at the variable level ranged from $1.5 \%(n=1)$ to $6.9 \%(n=4)$. 
At the case (i.e., observer) level, between 3.6\% $(n=1)$ and $14.3 \%(n=4)$ of the data, on average, was missing. Only six observers missed more than $15 \%$ of the items in a given video selection (i.e., they missed between $17.9 \%[n=5]$ and $21.4 \%[n=6]$ of the items in one of the questionnaires). However, there were nine participants who chose not to watch one of the videos that was presented to them (e.g., Participant \#011 skipped the video of Walker \#002, Participant \#061 skipped watching Walker \#004, etc.). Therefore, the Victim Vulnerability Rating Questionnaire was entirely blank for nine cases.

The missing data was examined for patterns. First, patterns of "missingness" were investigated at the variable level. The variables that had missing data were compared to variables that had complete data; participants did not tend to skip or miss certain variables more than others (i.e., the missing data was spread out across different variables). Each variable with missing data was also compared to the same variable in a different video series (e.g., the amount of data missing on the item that asked participants to rate the sexual vulnerability of Walker \#011 was compared to the amount of data missing on the item that rated the sexual vulnerability of Walker \#022). There was no discernible pattern observed across videos.

The missing data was then examined for patterns at the case level. The majority of the questionnaires were non-significant for Little MCAR's Test ${ }^{29}$ and had less than $15 \%$ of the data missing. Furthermore, the videos that were skipped, and the participants who skipped them, were all different from one another. Although it is possible that the missing data were related to an unmeasured factor (e.g., the observers' personality, affect, and/or

\footnotetext{
${ }^{29}$ Little MCAR's Test was significant for less than $25 \%$ of the questionnaires (e.g., the Victim Vulnerability Rating Questionnaire for Walker $\# 004$ was significant, $\chi^{2}[146]=177.90, p=.037$ ).
} 
their own victimization history), it was likely the result of participant fatigue and/or attrition. Therefore, the missing data was determined to be "missing at random."

Given that the analyses were to be conducted by video, and the number of observers who saw each video varied due to the randomization process, pair-wise deletion was conducted; the nine questionnaires that had been skipped entirely by participants, as well as those questionnaires that were missing over $15 \%$ of their data, were removed. For example, originally a sample of 65 observers had been randomly selected to view the video clip of Walker \#024 and complete its accompanying questionnaire. However, because Participant \#019 missed 21.4\% $(n=6)$ of the items in the questionnaire, they were removed from all analyses pertaining to Walker \#024. After deletion, the sample size for the Victim Vulnerability Rating Questionnaire for Walker \#024 was reduced to 64 . However, Participant \#019 was retained in the dataset for all other analyses (i.e., their complete data on the other Victim Vulnerability Rating Questionnaires [for the other walkers] was still used). Pair-wise deletion allowed the researcher to maximize all of the available data and retain power. However, because the sample sizes varied across videos and analyses, standard errors may have been over- (or under) estimated. Therefore, the results should be interpreted cautiously.

For the variables missing less than $15 \%$ (i.e., between 1 and 4 items) of the data, expectation maximization (EM) was employed. Although EM may underestimate standard errors (Allison \& Oaks, 2002), the data file (which consisted of multiple, randomized questionnaires) did not allow for other multiple imputation or Monte Carlo techniques to be conducted. Expectation maximization was chosen as a superior technique to mean substitution. Given the few missing data points, it is unlikely that 
significantly different results would have been obtained with a different method. The standard 25 iterations were conducted, and complete data was obtained for all 28 questionnaires. After pair-wise deletion and EM was performed, each questionnaire had a sample size ranging from 56 to 65 participants.

Outliers. Study 2 was conducted using the online survey tool, Qualtrics.

Therefore, participants were forced to choose a score within a specified range and they could not provide their own, out-of-range, score. The potential for human error (during data entry) was also eliminated since the data-set was computer-generated. Nevertheless, minimum and maximum scores were examined as a precaution given that EM had been conducted; approximately five outliers were detected and corrected. For the ordinal variables, z-scores were requested. Consistent with Van Selst and Jolicoeur's (1994) recommendations for small sample sizes, the majority of scores were under $\pm 2.50 .{ }^{30}$ However, approximately nineteen items exceeded the cut-off value and required correction.

Each of the scores that grossly exceeded \pm 2.50 were winsorized. For example, on the "Upset" item (which pertained to Walker\#014), the original z-score was 3.77. The one outlying score of 10 was winsorized to become 7 , which was the next highest score on that variable. After the outliers had been winsorized, all of the z-scores were reexamined. Several z-scores still remained above the approximate cut-off value (e.g., the item which asked participants to rate the hostility of Walker \#020 retained a z-score of 3.69). However, because the remaining outliers were extreme, but valid scores, they were left as is in the data-set.

\footnotetext{
${ }^{30}$ The dispersion of scores was also consistent with Field (2009); the majority of scores were under \pm 1.96 , very few scores reached \pm 2.58 , and only seven items (across all 28 questionnaires) exceeded the \pm 3.29 cut-off.
} 
Normality. The data-set for Study 2 consisted of more than 800 variables (i.e., the same 28-item Victim Vulnerability Rating Questionnaire and Demographic Questionnaire was completed for all 28 walker videos). Therefore, it was most feasible to examine skewness and kurtosis on the variables in one questionnaire (pertaining to one particular walker). Given that the items were the same for every walker, it may be assumed that similar normality would be obtained for the other 27 questionnaires. However, caution must be exercised when interpreting the results; future research may wish to formally examine skewness and kurtosis on all of the variables.

The Victim Vulnerability Rating Questionnaire for Walker \#015 was randomly selected, and skewness and kurtosis was calculated for all of the ordinal variables. As expected, some of the variables which asked participants to rate the vulnerability of the target to sexual and/or violent victimization appeared positively skewed. Given the sensitive nature of these questions (ratings of vulnerability), it is possible that some participants felt awkward providing answers that were not socially desirable/acceptable (i.e., a social desirability bias may have affected the shape of the distribution). Formal skew and kurtosis values were thus calculated for the variables of concern. The only variable which exceeded \pm 1.96 asked participants to rate "how easy a target" Walker $\# 015$ appeared to be $($ Skew $=2.01 ;$ Kurtosis $=-.67)$. The majority of observers provided low ratings (i.e., they indicated that the walker was not an easy target). The "Easy Target" item was examined across the other questionnaires to determine if the variable was also skewed elsewhere. The histograms and boxplots suggested that the distribution 
appeared relatively normal on all of the other questionnaires, ${ }^{31}$ and the item was therefore left untransformed.

\section{Primary Analyses}

The areas of research pertaining to "Study 2: The 'Observers"” are reiterated in Table 26. The analyses used to examine each area are noted and described in more detail following the table.

Table 26

Study 2 Analyses

Areas of Research Analysis

1) Consensus

2) Accuracy

Analysis

3) The effect of the observers' perceptions of the walkers' personality and affect on ratings of vulnerability

4) The effect of the walkers' own (self-reported) personality and affect on likelihood to experience victimization
Consensus Correlations

Correlations

Receiver Operating Characteristic

Linear Regression

Logistic Regression

To gain a better understanding of the ability of observers to detect nonverbal cues of vulnerability, two types of accuracy were assessed: consensus (i.e., the agreement among observers) and self-other agreement (i.e., the agreement between observer ratings and walker ratings). It was expected that the observers would agree (i.e., reach

\footnotetext{
${ }^{31}$ Five of the other questionnaires presented with slight skewness on the "easy target" item. However, none of the questionnaires had skewness values above 2.00 .
} 
consensus) on which walkers were most likely to be victimized, and that relatively strong self-other agreement accuracy would also be obtained.

Consensus. Consensus correlations, wherein a small group of the observer's ratings were correlated with the remaining group of observer's ratings, were calculated for all of the major variables in question. The items that were used to determine whether or not observers agreed on the vulnerability of the walkers included: "Easy Target," "Rating of Vulnerability to Sexual Victimization," and "Rating of Vulnerability to Violent Victimization." It was not feasible to ask the observers to complete the entire IASR-B5 or PANAS-X for each of the walkers they rated; thus, the walkers were only rated on certain items/adjectives of interest that were gleaned from the two measures.

To assess the perceived dominance of the walkers, the observers were asked to rate the walkers on their "Assertiveness" and "Self-confidence." Alternatively, to assess the perceived submissiveness of the walkers, the observers were asked to rate how "Timid" and "Unaggressive" they appeared. In relation to positive affect, the following items were assessed: "Alert," "Cheerful," "Determined," "Happy," and "Strong." In relation to negative affect, "Afraid," "Hostile," "Sad," and "Upset," were assessed. There were five additional items (i.e., "Capable," "Exploitable," "Independent," "Protection," and "Weak"), which were also assessed for descriptive purposes.

Given that the number of observers rating each target on the items outlined above ranged from 56-65, standard intra-class correlations (ICC's), which typically measure agreement between just two raters, could not be used. Variance Component analysis was also systematically ruled out as a calculation option for consensus; the set-up of the dataset made this approach a poor fit for the current study (i.e., the observers and the 
walkers could not be entered as "random factors" given how the data was arranged in SPSS). Therefore, a method conducted in Yeagley et al.'s (2007) study, which obtains agreement correlations when there is multiple raters, was used in the present study. Consistent with Yeagley et al. (2007), six observers (male and female) were chosen at random. The ratings of the group of six were averaged and subsequently correlated (using Pearson's $r$ ) with the average of the remaining observer's ratings; this process (whereby new randomly selected raters were chosen) was repeated for each item examined.

Recall that it was expected that observers would agree on which walker(s) were most vulnerable to victimization. The bivariate correlation for "Easy Target" was significant, $r=.32, p=.048$, and indicated that observers agreed on which walkers were easy targets. Next, consensus was examined for the item that asked the observers to rate the vulnerability of the walkers to sexual victimization. A separate group of six observers were chosen at random. The ratings of the new group were averaged for the item "Rating of Vulnerability to Sexual Victimization.” The new averaged variable was then correlated with the average of the remaining observer's ratings. The correlation was significant, $r=$ $.45, p=.008$, and indicated that observers agreed on which walkers were most vulnerable to sexual victimization. Similarly, a consensus correlation was calculated for the item that asked the observers to rate the vulnerability of the walkers to violent victimization (i.e., "Rating of Vulnerability to Violent Victimization"). The Pearson correlation was marginally significant, $r=.30, p=.061$. These findings suggested that while observers may not agree on which walkers are most vulnerable to violent victimization, they do tend to agree on which individuals are easy targets overall, as well as which individuals are vulnerable to sexual victimization. 
To determine whether observers also agreed with one another on which individuals were submissive and which exhibited the highest negative affect, consensus correlations were calculated for the items that asked about the perceived affect and perceived personality of each target walker. It was expected that observers would agree on which walkers were most likely to be submissive and which experience the highest negative affect. Table 27 provides a summary of the consensus correlations.

The consensus correlation indicated that the observers agreed on which walkers appeared the most self-confident, $r=.38, p=.024$. However, the correlation between the observers on the assertiveness item did not reach significance, $r=-.20, p=.160$. The discrepancy between the items is unclear given that both self-confidence and assertiveness (among others) provide an overall measure of assured-dominance in the IASR-B5. It is possible that a social desirability bias among raters affected the consistency of the results. While self-confidence tends to carry a positive connotation and would likely receive more generous ratings, assertiveness is often perceived to be a less desirable trait (Kern, 1982). Raters may thus have agreed on walker self-confidence simply because they were apt to rate more walkers as highly confident overall. Indeed, the mean rating of walker self-confidence was significantly higher $(M=5.29, S D=.81)$ than the mean rating of walker assertiveness $(M=4.78, S D=.71), t(27)=9.98, p<.001$.

The observers' ratings of how timid and unaggressive the walkers appeared were both non-significant ( $r=.28, p=.071$ and $r=-.19, p=.163$, respectively). However, the item which asked the observers to rate how timid the walkers appeared approached significance. The results suggested that while observers may agree on the self-confidence 
of others, they are less able to collectively agree on their assertiveness, how timid they are, and how unaggressive they appear.

In regards to the affect of the walkers, observer consensus was examined for the items: Afraid, hostile, alert, cheerful, determined, happy, sad, upset, and strong. The observers only reached consensus on which walkers appeared strong, $r=.40, p=.017$. Strong is an indicator of General Positive Affect in the PANAS-X. Thus, it appears that zero-acquaintance observers may agree on which individuals appear most positive (based on 10 -seconds worth of gait movement). The consensus correlations for afraid $(r=.30, p$ $=.061)$, and sad $(r=.30, p=.061)$ approached significance. Afraid is a component of the Fear scale, and sad is a component of the Sadness scale. However, a larger sample size is necessary to ascertain whether or not observers truly agree on the apparent fear and sadness of others. 
Table 27

Consensus Correlations

\begin{tabular}{|c|c|c|}
\hline \multirow{2}{*}{ Items Rated by Observers } & \multicolumn{2}{|c|}{$\underline{\text { Observer Consensus }}$} \\
\hline & $r$ & $p$ \\
\hline \multicolumn{3}{|l|}{ Walker Personality Items } \\
\hline \multicolumn{3}{|l|}{ Dominance } \\
\hline Assertive & -.20 & .160 \\
\hline Self-Confident & .38 & $.024 *$ \\
\hline \multicolumn{3}{|l|}{ Submissiveness } \\
\hline Timid & .28 & .071 \\
\hline \multirow[t]{2}{*}{ Unaggressive } & -.19 & .163 \\
\hline & .28 & .071 \\
\hline \multicolumn{3}{|l|}{ Walker Affect Items } \\
\hline \multicolumn{3}{|l|}{ Negative Affect } \\
\hline Afraid & .27 & .081 \\
\hline Hostile & -.14 & .247 \\
\hline Sad & .26 & .087 \\
\hline Upset & .13 & .252 \\
\hline \multicolumn{3}{|l|}{ Positive Affect } \\
\hline Alert & -.10 & .312 \\
\hline Cheerful & .17 & .188 \\
\hline Determined & .05 & .395 \\
\hline Happy & .12 & .271 \\
\hline Strong & .40 & $.017^{*}$ \\
\hline \multicolumn{3}{|l|}{ Descriptive Items } \\
\hline Capable & -.11 & .294 \\
\hline Exploitable & .26 & .089 \\
\hline Independent & -.22 & .134 \\
\hline Protection & .14 & .246 \\
\hline Weak & .17 & .192 \\
\hline \multicolumn{3}{|l|}{ Vulnerability Items } \\
\hline Easy Target & .32 & $.048 *$ \\
\hline Sexual Vulnerability & .45 & $.008 *$ \\
\hline Violent Vulnerability & .30 & .061 \\
\hline
\end{tabular}

Note. Recall that due to issues with reflection and calibration, only 28 of the 31 videos were useable. Therefore, $N=28$ videos. $r=$ Pearson Correlation. $* p<.05$.

Accuracy. In terms of accuracy, it was hypothesized that relatively strong selfother agreement correlations would be observed between observer ratings of walker 
vulnerability to various types of victimization and walker self-reports of their own victimization history. Given that the majority of the scales were normally distributed, it was not surprising that the average vulnerability rating was approximately five (see Tables 28 and 29). Therefore, consistent with Book et al. (2013) observers were, “...considered to be accurate in their judgements if they gave 'non-victims' a vulnerability score between 1 and 5 , and if they gave 'victims' a vulnerability score between 6 and 10. The midpoint of the scale was used because the values from 1 to 5 described the person as not being vulnerable to victimization, while values from 6 to 10 described the targets as vulnerable to victimization" (p. 2375). ${ }^{32}$ An accurate rating by an observer received a coding of 1 and an inaccurate rating received a coding of 0 . The number of observers (for each walker) that were accurate in their ratings were then tallied.

As outlined in Tables 28 and 29, the majority of participants were relatively accurate in their judgements of the walkers' vulnerability to both violent (ratings were accurate on 18 out of the total 28 walkers) and sexual victimization (ratings were accurate on 15 out of the total 28 walkers). In other words, the observers were accurate in their ratings of the walkers' vulnerability to violent victimization $64 \%$ of the time. The observers were also accurate in their ratings of the walkers' vulnerability to sexual victimization $54 \%$ of the time. Interestingly, the observers appeared better able to distinguish those vulnerable to violent victimization, as only slightly over half of the sample were able to accurately distinguish those vulnerable to sexual victimization.

\footnotetext{
${ }^{32}$ For exploratory purposes, half of the sample was scored with the midpoint of the scale removed. The accuracy of the observers was compared when the midpoint was removed and when it was present. The findings suggested that if the score of " 5 " had been excluded from analyses, accuracy would have improved.
} 
Table 28

Accuracy of Observer Ratings of the Walkers' Vulnerability to Violent Victimization

\begin{tabular}{|c|c|c|c|c|c|c|}
\hline \multirow[t]{2}{*}{ Walker } & \multicolumn{4}{|c|}{$\begin{array}{c}\text { Observer Ratings of Violent } \\
\text { Victimization }\end{array}$} & \multirow{2}{*}{$\begin{array}{c}\text { Walker Violent } \\
\text { Victimization History } \\
\text { Yes/No }\end{array}$} & \multirow{2}{*}{$\begin{array}{c}\text { Accuracy of } \\
\text { the } \\
\text { Observers }^{\text {b }}\end{array}$} \\
\hline & Mode & Median & $M$ & $S D$ & & \\
\hline 001 & $6^{\mathrm{a}}$ & 6.00 & 6.03 & 2.54 & No & Inaccurate \\
\hline 002 & 7 & 5.00 & 5.22 & 2.74 & No & Accurate \\
\hline 003 & 10 & 5.50 & 5.53 & 2.99 & No & Accurate \\
\hline 004 & 3 & 5.00 & 4.90 & 2.75 & No & Accurate \\
\hline 005 & 7 & 5.00 & 4.72 & 2.66 & No & Accurate \\
\hline 006 & 4 & 5.00 & 5.08 & 2.51 & No & Accurate \\
\hline 007 & 7 & 5.00 & 5.18 & 2.83 & No & Accurate \\
\hline 008 & 1 & 3.00 & 3.97 & 2.60 & Yes & Inaccurate \\
\hline 009 & 7 & 7.00 & 6.40 & 2.74 & No & Inaccurate \\
\hline 010 & 1 & 4.00 & 4.09 & 2.64 & Yes & Inaccurate \\
\hline 011 & 1 & 3.50 & 4.09 & 2.60 & No & Accurate \\
\hline 014 & 1 & 5.00 & 5.08 & 2.91 & Yes & Inaccurate \\
\hline 015 & 1 & 4.00 & 4.23 & 2.51 & No & Accurate \\
\hline 016 & 3 & 3.00 & 4.12 & 2.73 & Yes & Inaccurate \\
\hline 017 & 3 & 4.00 & 4.28 & 2.32 & No & Accurate \\
\hline 018 & 1 & 5.00 & 5.03 & 2.79 & Yes & Inaccurate \\
\hline 020 & $5^{\mathrm{a}}$ & 6.00 & 5.75 & 2.77 & Yes & Accurate \\
\hline 021 & 1 & 5.00 & 4.44 & 2.82 & No & Accurate \\
\hline 022 & 1 & 4.00 & 3.92 & 2.40 & No & Accurate \\
\hline 023 & 5 & 5.00 & 5.09 & 2.79 & Yes & Inaccurate \\
\hline 024 & 7 & 6.00 & 5.33 & 2.46 & Yes & Accurate \\
\hline 025 & 1 & 3.50 & 3.97 & 2.58 & No & Accurate \\
\hline 026 & 5 & 6.00 & 5.63 & 2.79 & Yes & Accurate \\
\hline 027 & 1 & 3.00 & 3.41 & 2.44 & Yes & Inaccurate \\
\hline 028 & 2 & 3.00 & 4.21 & 2.77 & No & Accurate \\
\hline 029 & 1 & 3.50 & 3.80 & 2.55 & Yes & Inaccurate \\
\hline 030 & 5 & 5.00 & 5.09 & 2.67 & No & Accurate \\
\hline 031 & 1 & 5.00 & 4.71 & 2.86 & No & Accurate \\
\hline
\end{tabular}

Note. Recall that due to issues with reflection and calibration, only 28 of the 31 videos were useable. Therefore, $N=28$ videos.

${ }^{a}$ Multiple modes were present; the lowest value is presented. ${ }^{\mathrm{b}}$ The raw observer responses were recoded for every observers' score on every walker. If an observer scored a victimized walker as a 6 or above, then they received a " 1 " (i.e., accurate); if an observer scored a non-victimized walker between 1 and 5, they also received a " 1 " for accurate. Accurate ratings (i.e., " 1 's") were tallied for each walker (i.e., the number of " 1 's" present for each walker were summed), and then divided by the total number of observers who rated that walker. If the majority of observers were accurate (i.e., over 50\%) then they received a designation of "accurate" in the last column. 
Table 29

Accuracy of Observer Ratings of the Walkers' Vulnerability to Sexual Victimization

\begin{tabular}{|c|c|c|c|c|c|c|}
\hline \multirow[t]{2}{*}{ Walker } & \multicolumn{4}{|c|}{$\begin{array}{l}\text { Observer Ratings of Sexual } \\
\text { Victimization }\end{array}$} & \multirow{2}{*}{$\begin{array}{c}\text { Walker Sexual } \\
\text { Victimization } \\
\text { History }\end{array}$} & \multirow{2}{*}{$\begin{array}{l}\text { Accuracy of the } \\
\text { Observers }{ }^{b}\end{array}$} \\
\hline & Mode & Median & $M$ & $S D$ & & \\
\hline 001 & 8 & 6.00 & 5.85 & 2.64 & No & Inaccurate \\
\hline 002 & 5 & 5.00 & 5.14 & 2.84 & No & Accurate \\
\hline 003 & $1^{\mathrm{a}}$ & 5.00 & 5.03 & 2.99 & No & Accurate \\
\hline 004 & 1 & 4.00 & 4.55 & 2.76 & No & Accurate \\
\hline 005 & 5 & 5.00 & 4.38 & 2.68 & No & Accurate \\
\hline 006 & 5 & 5.00 & 4.46 & 2.47 & Yes & Inaccurate \\
\hline 007 & 1 & 4.50 & 4.80 & 2.81 & Yes & Inaccurate \\
\hline 008 & 1 & 3.00 & 3.92 & 2.72 & No & Accurate \\
\hline 009 & 5 & 6.00 & 5.92 & 2.82 & No & Inaccurate \\
\hline 010 & 1 & 3.00 & 3.69 & 2.65 & Yes & Inaccurate \\
\hline 011 & 1 & 3.00 & 3.92 & 2.52 & No & Accurate \\
\hline 014 & 1 & 6.00 & 5.12 & 2.90 & Yes & Accurate \\
\hline 015 & $1^{\mathrm{a}}$ & 4.00 & 4.02 & 2.55 & No & Accurate \\
\hline 016 & 1 & 3.00 & 4.06 & 2.68 & Yes & Inaccurate \\
\hline 017 & 1 & 4.00 & 4.23 & 2.36 & No & Accurate \\
\hline 018 & 1 & 4.50 & 4.64 & 2.96 & No & Accurate \\
\hline 020 & 5 & 5.00 & 5.52 & 2.80 & No & Accurate \\
\hline 021 & 1 & 4.50 & 4.36 & 2.88 & No & Accurate \\
\hline 022 & 1 & 3.00 & 3.71 & 2.50 & Yes & Inaccurate \\
\hline 023 & 1 & 4.50 & 4.64 & 2.83 & Yes & Inaccurate \\
\hline 024 & 5 & 5.00 & 4.81 & 2.31 & Yes & Inaccurate \\
\hline 025 & 1 & 3.00 & 3.73 & 2.61 & Yes & Inaccurate \\
\hline 026 & 7 & 6.00 & 5.66 & 2.77 & No & Inaccurate \\
\hline 027 & 1 & 3.00 & 3.80 & 2.57 & Yes & Inaccurate \\
\hline 028 & 2 & 3.00 & 4.21 & 2.67 & Yes & Inaccurate \\
\hline 029 & 1 & 3.00 & 3.45 & 2.43 & No & Accurate \\
\hline 030 & $1^{\mathrm{a}}$ & 4.00 & 4.70 & 2.75 & No & Accurate \\
\hline 031 & $1^{\mathrm{a}}$ & 4.00 & 4.44 & 2.75 & No & Accurate \\
\hline
\end{tabular}

Note. Recall that due to issues with reflection and calibration, only 28 of the 31 videos were useable. Therefore, $N=28$ videos.

${ }^{a}$ Multiple modes were present; the lowest value is presented. ${ }^{\mathrm{b}}$ The raw observer responses were recoded for every observers' score on every walker. If an observer scored a victimized walker as a 6 or above, then they received a "1" (i.e., accurate); if an observer scored a non-victimized walker between 1 and 5, they also received a "1" for accurate. Accurate ratings (i.e., " 1 's") were tallied for each walker (i.e., the number of " 1 's" present for each walker were summed), and then divided by the total number of observers who rated that walker. If the majority of observers were accurate (i.e., over 50\%) then they received a designation of "accurate" in the last column. 
Although it appears as if the observers were relatively accurate in selecting past victims as future victims based on movement cues, the base rate of the sample must be considered. Given that there were eleven victims of violent crime, and seventeen individuals who were not violently victimized, observers who rated the majority of the walkers as not vulnerable to violent victimization had an advantage in terms of rating accuracy. Similarly, eleven individuals had been sexually victimized, and seventeen walkers did not have a history of sexual victimization. Therefore, observers who provided low ratings of vulnerability for the majority of the walkers appeared accurate. As illustrated in the above tables, most ratings deemed "accurate" were for walkers who had not been victimized, and who had received a low vulnerability score from the observers.

To further examine the issue of base rate, individuals with any type of previous victimization history (sexual and/or violent) were examined relative to the observers' ratings on the item "Vulnerability to Any Victimization." When both types of victimization were combined, approximately $57.1 \%(n=16)$ of the walkers had a previous victimization history, and $42.9 \%(n=12)$ had no prior victimization experiences. Accuracy rates were tallied using the new base rate. When there were more victimized than non-victimized individuals in the sample, observer accuracy disappeared. The majority of observers were accurate in their ratings of walker vulnerability on only twelve out of the total twenty-eight walkers (i.e., the observers were accurate in their ratings of the walkers' vulnerability to victimization $43 \%$ of the time); accuracy levels were below chance.

Although percent accuracy calculations (such as those described above) provide some indication as to the number of observers who scored the walkers correctly, the 
results are dependent on the threshold chosen (for defining when a target is deemed "vulnerable"). If one changed the threshold for determining what a "correct" score is, then the percent accuracy could shift. For example, perhaps some observers do score individuals with victimization histories higher on their vulnerability to victimization, but only up to the 3 or 4 scale range. These higher ratings would not be accounted for because observers were only determined to be "correct" if they provided self-reported victims a vulnerability score between 6 and 10, and self-reported non-victims a vulnerability score between 1 and 5. Because the base rate is low (i.e., observers tended to score the majority of walkers low on their vulnerability to victimization) any changes in ratings at the bottom end of the scale, would not be accounted for (i.e., because accuracy has been established by dichotomizing [i.e., splitting the top and bottom half] a scale variable).

Receiver Operating Characteristic (ROC) analysis allows one to evaluate the discriminatory ability of raters (e.g., to correctly select those with a victimization history as those most vulnerable to future victimization; e.g., Kumar \& Indrayan, 2011; Swets, 1988). A graph is produced, whereby "true positives" (i.e., deciding that a walker is vulnerable, when they have in fact been previously victimized) are plotted against "false positives" (i.e., deciding that a walker is vulnerable when they have not been previously victimized) at every level of the vulnerability scale. Each threshold (one point per threshold) becomes a point on the graph that, when connected, forms a concave downward curve (a ROC curve).

A measure of discriminatory ability can then be calculated by measuring the area of the ROC graph falling beneath the ROC curve (referred to as the area under the curve, 
or the AUC). The AUC ranges from .5 to 1.00, where an AUC of .5 is no better than chance and 1.00 refers to perfect discrimination accuracy (i.e., observers consistently rate those with previous victimization histories as those most vulnerable to future victimization). Unlike the approach used above (i.e., percent accuracy), the AUC is not biased by the threshold used by observers to determine vulnerability (because the AUC reflects the position of the entire ROC curve in its graph, rather than any single point [threshold] along that curve). Furthermore, the AUC is not biased by the base rate of what is being predicted (i.e., previously victimized walkers) because the ROC graph is based on the proportion of the decision outcomes (i.e., true positive and false positive) rather than by the frequency of victimized (versus non-victimized) walkers.

Between 56 and 65 observers rated each walker on their vulnerability. Therefore, the observers' scores were pooled for every walker (e.g., the raw scores from the sample of observers who rated Walker \#10 were averaged, the raw scores from the sample of observers who rated Walker \#20 were averaged, etc.). The pooled observers' scores were used to predict (i.e., they were entered as the outcome variable in the ROC analysis) the actual self-reported victimization history of the walkers (dichotomously scored as yes or no; i.e., the "test" variable).

Three ROC curves were constructed. The first curve estimated how accurate the observers were in their ratings of the walkers' vulnerability to violent victimization. Accuracy was poor $(A U C=.56, S E=.12,95 \%$ CI $[.32, .79], p=.621)$ according to criteria proposed by Swets (1988). ${ }^{33}$ This finding suggests that observers did not

\footnotetext{
${ }^{33}$ According to Swets (1988), AUCs below .50 are non-informative, AUCs between .50 and .70 indicate low accuracy, AUCs between .70 and .90 indicate moderate accuracy, and AUCs between .90 and 1 indicate high accuracy (also see Bennell, Mugford, Ellingwood, and Woodhams, 2013, for a discussion of these guidelines).
} 
discriminate between those with a history of violent victimization and those without a history of violent victimization any better than chance.

The second curve estimated how accurate the observers were in their ratings of the walkers' vulnerability to sexual victimization. Once again, accuracy was poor $(A U C=$ $.66, S E=.11,95 \% \mathrm{CI}[.45, .87], p=.158)$. This finding suggests that, although observers were more accurate at discriminating between walkers with and without histories of sexual victimization (compared to those with violent victimization histories), they still failed to discriminate at a significant level (i.e., at $p<.05$ ).

The final curve estimated how accurate the observers were in their ratings of the walkers' vulnerability to any type of victimization. Accuracy was also poor for this final model $(A U C=.65, S E=.11,95 \% \mathrm{CI}[.44, .85], p=.186)$; observers performed poorly at detecting those with any (violent and/or sexual) history of victimization. The results of the three ROC analyses confirmed that the observers were inaccurate, even when the issue of base rate was remedied. In other words, observers did not rate the walkers with self-reported histories of victimization as more vulnerable.

Based on previous research (e.g., Book et al., 2013), it was expected that individuals who exhibited a vulnerable walk would also self-report past victimization experiences. However, because some individuals exhibited the vulnerable gait pattern, but did not report a history of victimization, Spearman correlations were calculated between the observers' (averaged) ratings of the walkers' vulnerability (an ordinal scale variable ranging from $1=$ not at all vulnerable to $10=$ completely vulnerable) and the coded gait patterns (an ordinal scale variable; $0=$ no vulnerability features present in movement; 5 = all vulnerability features present in movement). 
The correlation between the averaged observers' ratings of the walkers' vulnerability to sexual victimization and gait pattern was significant, $r_{s}=.33, p=.046$. Similarly, the correlation between the averaged observer ratings of the walkers' vulnerability to violent victimization and gait pattern was significant, $r_{s}=.37, p=.028$. The results suggested that, on average, observers provided higher vulnerability ratings to the walkers with more vulnerable features present in their gait. Given that the observers were able to differentiate between vulnerable and non-vulnerable gait movement, the ratings were considered accurate.

Lastly, recall that observer ratings were not predicted to necessarily correlate accurately with the walkers' self-reported submissiveness and affect. Self-other agreement correlations (using Spearman's Rank-Order Correlation) were calculated between the observers' perceptions of the walkers' personality and walkers' affect (ordinal variables rated on a 10-point scale) and the walkers' self-reports of their own personality and affect (scale scores from the IASR-B5 and PANAS-X). As expected, selfother agreement accuracy was not obtained for any of the items (all correlations were $<p$ .05 ; see Table 30 for a summary of the self-other agreement correlations). Thus, it appears that the way one perceives them self may not be how a stranger perceives them. Interestingly, however, the results suggested that the walkers who rated themselves high on the negative affect items (i.e., sad and upset) received significantly lower ratings of negative affect by observers $\left(r_{s}=-.43, p=.010\right.$ and $r_{s}=-.39, p=.019$, respectively). The present findings conflict with previous research (e.g., Schneider et al., 2014), which suggests that zero-acquaintance observers are particularly sensitive to displays of negative affect in others. 
Table 30

Self-other Agreement Correlations

Self-other Agreement

$\underline{\text { Items Rated by Observers }}$

$r_{s} \quad p$

Walker Personality Items

Assertive

Timid

Self-confident

Unaggressive

.19

$-.24$

$-.24$

$-.14$

.171

.105

.113

.234

Walker Affect Items

Afraid

.05

.24

$-.27$

$-.17$

$-.08$

$-.28$

$-.43$

$-.39$

$-.23$
.395

.112

.081

.191

.346

.074

$.010 * *$

$.019 *$

.123

Note. Recall that due to issues with reflection and calibration, only 28 of the 31 videos were useable. Therefore, $N=28$ videos. $r_{s}=$ Spearman Correlation. $* p<.05$. $* * p<.01$.

For exploratory purposes, differences in accuracy were examined for male and female observers across all of the items (i.e., the vulnerability items, personality items, and affect items). The chi-square tests conducted for each walker suggested that there were no significant differences in accuracy between male and female observers for the majority of the walkers. A significant difference in accuracy was observed for only five of the walkers; of these five walkers, on average, male observers were more accurate in their ratings of walker vulnerability (i.e., male observers were more likely to rate walkers with past self-reported victimization experiences as those most vulnerable to future victimization). The chi-square results are illustrated in Table 31. 
Table 31

Chi-square Statistics Examining Differences in Rating Accuracy for Male and Female Observers

\begin{tabular}{|c|c|c|c|c|}
\hline Walker & Observers & Wrong & Right & Chi-square Test \\
\hline 003 & Females & $72.7 \%$ & $41.7 \%$ & \multirow{2}{*}{$\begin{array}{c}\chi(1)=4.51, p \\
=.033, \mathrm{Phi}= \\
.31\end{array}$} \\
\hline $\begin{array}{c}\text { Violent } \\
\text { Vulnerability }\end{array}$ & Males & $27.3 \%$ & $58.3 \%$ & \\
\hline 007 & Females & $75 \%$ & $38.5 \%$ & \multirow{2}{*}{$\begin{array}{c}\chi(1)=6.08, p \\
=.014, \text { Phi }= \\
.36\end{array}$} \\
\hline $\begin{array}{c}\text { Violent } \\
\text { Vulnerability }\end{array}$ & Males & $25 \%$ & $61.5 \%$ & \\
\hline 020 & Females & $73.7 \%$ & $29.2 \%$ & \multirow{2}{*}{$\begin{array}{c}\chi(1)=8.41, p \\
=.004, \mathrm{Phi}= \\
.44\end{array}$} \\
\hline $\begin{array}{c}\text { Sexual } \\
\text { Vulnerability }\end{array}$ & Males & $26.3 \%$ & $70.8 \%$ & \\
\hline 020 & Females & $23.8 \%$ & $72.7 \%$ & \multirow{2}{*}{$\begin{array}{c}\chi(1)=10.29, p \\
=.002, \text { Phi }=- \\
.49\end{array}$} \\
\hline $\begin{array}{c}\text { Violent } \\
\text { Vulnerability }\end{array}$ & Males & $76.2 \%$ & $27.3 \%$ & \\
\hline $\begin{array}{c}030 \\
\text { Violent }\end{array}$ & Females & $71.4 \%$ & $42.9 \%$ & \multirow{2}{*}{$\begin{array}{c}\chi(1)=3.96, p \\
=.044, \mathrm{Phi} \\
=.28\end{array}$} \\
\hline Vulnerability & Males & $28.6 \%$ & $57.1 \%$ & \\
\hline
\end{tabular}

Note. Recall that due to issues with reflection and calibration, only 28 of the 31 videos were useable. Therefore, $N=28$ videos. Between 56 and 65 observers rated each walker on their vulnerability. Approximately $55.3 \%(n=52)$ of the total sample of observers were female and $44.7 \%(n=42)$ were male.

\section{The effect of the observers' perceptions of the walkers' personality and affect}

on ratings of vulnerability. Based on previous research (e.g., Sakaguchi \& Hasegawa, 2006), it was expected that observer perceptions of walker personality and/or affect would predict ratings of vulnerability. More specifically, it was expected that perceptions of walker submissiveness and/or negative affect, would result in higher ratings of vulnerability (i.e., walkers that appeared to be submissive or experiencing negative affect, would be rated as more vulnerable to victimization). 
To examine the effect of the observers' perceptions of the walkers' personality and affect on ratings of vulnerability two regression analyses were conducted. The first regression used the observers' ratings of the walkers' vulnerability to violent victimization as the outcome. The second regression used the observers' ratings of the walkers' vulnerability to sexual victimization as the outcome variable. Both regressions used the personality and affect variables as the predictors. More specifically, the predictors in the original models included the four personality items (i.e., assertive, timid, self-confident and unaggressive), as well as the nine affect items (i.e., afraid, hostile, alert, cheerful, determined, happy, sad, upset, and strong).

As expected, given that the various adjectives in the Victim Vulnerability Rating Questionnaire were measuring similar characteristics, the original models presented with multicollinearity. Therefore, the adjectives were combined to create an overall “submissive personality" variable (comprised of "timid" and "unaggressive"), a “dominant personality" variable (comprised of "assertive" and "self-confident"), a "negative affect" variable (inclusive of the adjectives "upset," "sad," "afraid," and "hostile)," and a "positive affect" variable (inclusive of the adjectives "strong," "alert," "cheerful," "happy," and "determined)." The Variance Inflation Factor in the new models remained under 3 , Tolerance was well above .3 on all of the predictors, the Pearson bivariate correlations (examining the relationships among the predictors) were not significant, and the Condition Index remained under 10. Thus, the assumption of collinearity was no longer violated.

Given that multiple items were combined to create new variables for the regression analyses, normality was re-examined. Histograms with a fitted normal curve 
were requested for "submissive personality," "dominant personality," "positive affect," and "negative affect;" the graphs appeared normal. Skewness and kurtosis values remained under the cut-off of \pm 1.96 , and the Kolmogorov-Smirnov test was not significant $(p>.200)$ on the variables included in the analyses. Therefore, it appeared that the data satisfied the assumption of normality.

To ensure that there was a linear relationship present between each of the predictors and the outcome, several scatterplots were created. Visual inspection of the scatterplots suggested that linearity could be assumed (see Figures 4 and 5). The scatterplots were also examined for evidence of heteroscedasticity; the error terms along the line of best fit appeared relatively equal and the Q-Q Plots of the standardized residuals were homogeneous. Levene's Test was also not significant at the $p<.05$ level for any of the variables included in the analyses.

To test for independence of observation, the Durbin-Watson statistic was obtained. None of the models exceeded 2.5; it was thus assumed that autocorrelation was not a concern in the present analyses. Lastly, given that four predictors were to be entered into each regression model (i.e., submissive personality, dominant personality, positive affect, and negative affect), the data was examined for multivariate outliers. Mahalanobis Distance was requested and values were sorted and compared against the critical value of 18.5 at $p<.001(d f=4)$; no outliers exceeded the critical value and all cases remained in the dataset.

Recall that the first linear regression analysis conducted examined the extent to which the observers' ratings of the walkers' personality and affect predicted their vulnerability to violent victimization (i.e., did the observers' perceptions of the walkers' 
personality and affect have an effect on how they would rate their vulnerability to violent victimization?). The observers' mean ratings of each walkers' perceived submissiveness, dominance, positive affect, and negative affect, were entered as the predictors; ${ }^{34}$ the observers' mean rating of each walkers' vulnerability to violent victimization was designated as the outcome. The model was significant and the predictors explained $77.1 \%$ of the variance in vulnerability ratings, $\mathrm{R}^{2}=.771, F(4,27)=19.41, p<.001$. Thus, the observers' perceptions of the walkers' personality and affect significantly predicted their ratings of the walkers' vulnerability to violent victimization.

The only regression coefficient that reached statistical significance was the aggregated "Submissive Personality" variable $(b=.93, S E=.19)$. This finding indicates that, for every one unit increase in walker submissiveness, perceived vulnerability to violent victimization increased by .93 units. Or, in other words, on average, the walkers who were perceived to be submissive (i.e., timid and unaggressive) were rated by the observers as significantly more vulnerable to violent victimization relative to others, holding all else constant, $t(27)=5.00, p<.001$. The scatterplot displayed in Figure 3 (below) illustrates the relationship between submissive personality (perceived by observers) and the observers' mean rating of each walkers' vulnerability to violent victimization.

\footnotetext{
${ }^{34}$ All predictors were entered simultaneously (i.e., the forced entry method was used). Field (2009), as well
} as Studenmund and Cassidy (1987), argue that forced entry is most appropriate for theory testing. 


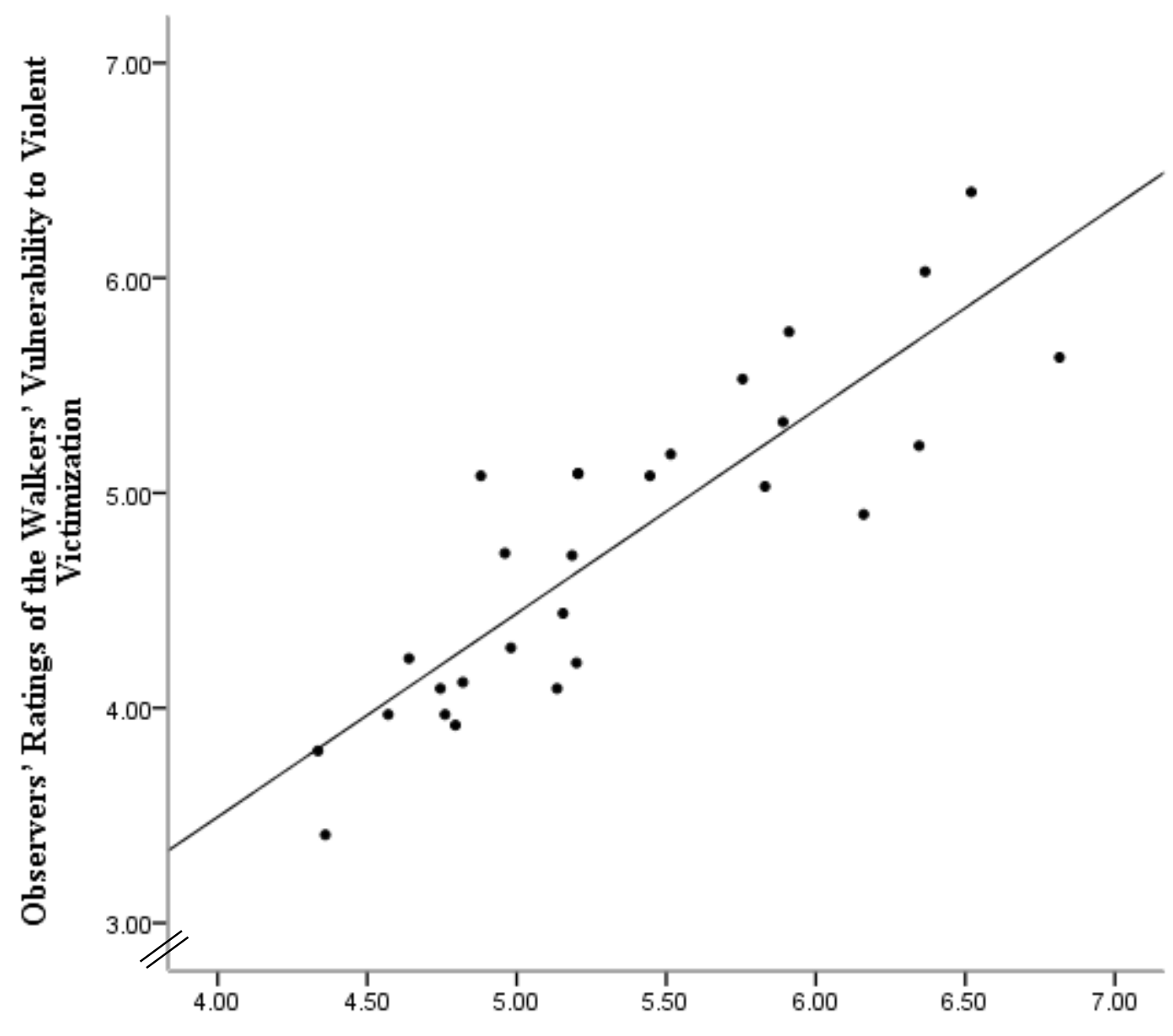

\section{Submissive Personality}

Figure 3. Relationship between the perceived submissiveness of the walkers and the observers' judgements of each walkers' vulnerability to violent victimization

The second linear regression analysis conducted examined the extent to which the perceived personality and affect of the walkers predicted their vulnerability to sexual victimization (i.e., did the observers' ratings of the walkers' personality and affect predict how they would rate the walkers' vulnerability to sexual victimization?). Once again, the model was significant and explained $79.6 \%$ of the variance in vulnerability ratings, $\mathrm{R}^{2}=$ $.796, F(4,27)=22.42, p<.001$. The observers' perceptions of the walkers' personality and affect significantly predicted their ratings of the walkers' vulnerability to sexual victimization. 
The regression coefficient that reached significance was once again "Submissive Personality" $(b=-.85, S E=.17)$. Interestingly, however, the finding indicated that, for every one unit increase in walker submissiveness, perceived vulnerability to sexual victimization decreased by .85 units. Or, in other words, on average, walkers who were perceived to be submissive (i.e., timid and unaggressive) were rated by the observers as significantly less vulnerable to sexual victimization relative to others, holding all else constant, $t(27)=-4.86, p<.001$. The scatterplot displayed in Figure 4 (below) illustrates the relationship between submissive personality (perceived by observers) and the observers' mean rating of each walkers' vulnerability to sexual victimization.

It appears that while observers perceive potential victims of violent crime to be highly submissive, they do not tend to rate submissive individuals highly on their vulnerability to sexual victimization. Although the finding may appear counterintuitive, recall from Study 1 that there was a lack of association observed between sexual victimization and gait; only those who had been violently victimized were significantly more likely to exhibit a vulnerable gait pattern. It is thus possible that only those individuals who exhibit the vulnerable gait pattern are perceived by others to be submissive. As noted by Ritchie (2014), it may be the case that observers use different and/or additional cues (over and above gait) to make judgements regarding one's vulnerability to sexual victimization. 


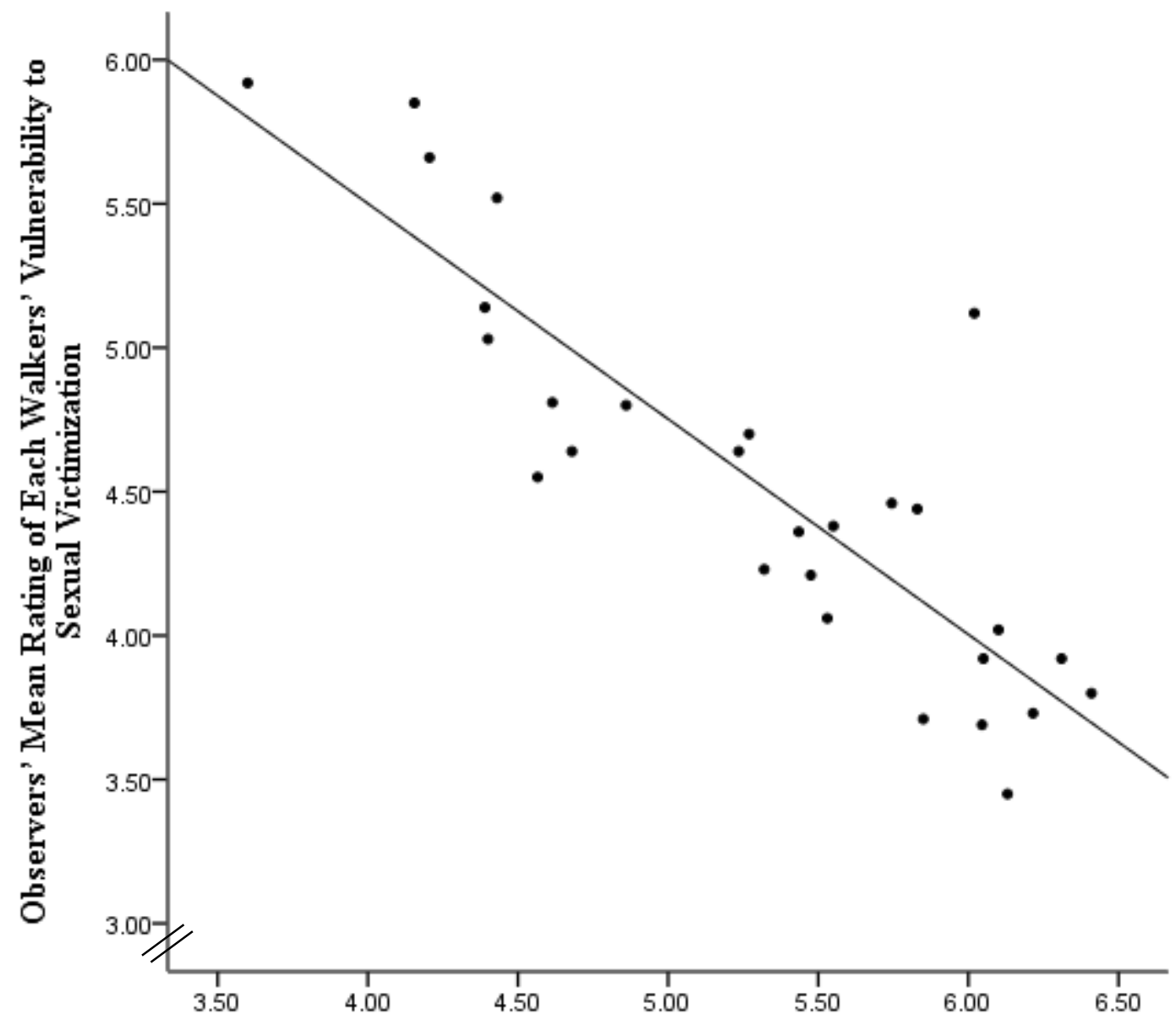

Submissive Personality

Figure 4. Relationship between the perceived submissiveness of the walkers and the observers' judgements of each walkers' vulnerability to sexual victimization

\section{The effect of the walkers' own (self-reported) personality and affect on}

likelihood to experience victimization. In an attempt to further understand the effect of personality and affect on victimization, an exploratory logistic regression analysis was conducted. In order to remain consistent with the observers' ratings of the walkers' personality and affect in the prior regression analyses, the four condensed (face-value) measures (i.e., "Submissive Personality" [inclusive of the adjectives 'timid,' and 'unaggressive'], “Dominant Personality” [inclusive of the adjectives 'self-confident,' and 'assertive'], 'Negative Affect" [inclusive of the adjectives 'upset,' 'sad,' 'afraid,' and 
'hostile'], and "Positive Affect" [inclusive of the adjectives 'strong,' 'alert,' 'cheerful,' 'happy,' and 'determined']) were pulled from the walkers' self-reports and used as the predictor variables in the current regression analysis; the walkers' victimization history was entered as the outcome variable.

Like linear regression, logistic regression assumes linearity, collinearity, and independence of errors. However, linearity differs in that each predictor is assumed to have a linear relationship with the log of the outcome variable. Therefore, interaction terms were computed; each term included one of the predictor variables (e.g., "submissive personality") and the log of itself (e.g., "LN submissive personality"). The regression model was then requested, and the main effects, as well as the interactions, were examined. The interactions were not significant at $p<.05$, and it could be assumed that linearity of the logit was satisfied.

The model predicting sexual victimization history from the walkers' self-reported personality and affect was not significant. However, the model predicting violent victimization history was significant, $\chi^{2}(4)=15.27, p=.004$; Nagelkerke's $R^{2}=.570$, suggesting that the walkers' self-reported personality traits and affective states were relatively good predictors of one's violent victimization history. ${ }^{35}$ The analyses revealed that "Dominant Personality" significantly predicted the likelihood of violent victimization. More specifically, on average, as self-reported dominance increased, the odds of having a violent victimization history declined. Or, in other words, those who indicated that they had been violently victimized were significantly less likely to have a dominant personality (i.e., they reported less self-confidence and assertiveness), $b=$ $2.46, p=.018, \operatorname{Exp}(\mathrm{B})=.09$.

${ }^{35}$ Hosmer and Lemeshow's Test indicated that the model fit appropriately, $\chi^{2}(7)=10.95, p=.141$. 
Given the findings in Study 1, which suggested that Assured-Dominance was significantly related to gait, and not associated with victimization history, the present findings are intriguing. Additional research is necessary in order to disentangle the various relationships between the observers' perceptions of the walkers' personality and affect, and the walkers' own self reports of their personality and affect, as well as their association with gait. As it was not feasible to ask the observers to complete the entire IASR-B5 or PANAS-X for each of the walkers they rated, adjectives were selected from each of the main scales of interest to act as measures of "face-value" for that particular state or trait (e.g., "sad" was selected to represent the entire "Sadness" scale in the PANAS-X). Unfortunately, the reliability of these items is unclear and different results may have been obtained had the observers rated the walkers using the complete measures. Nevertheless, there appears to be an enduring relationship between victimization, dominance, and gait; the precise nature of which remains unknown.

\section{Discussion}

\section{Consensus}

Study 2 consisted of four major areas of research. The first hypothesis aimed to determine the extent to which observers collectively agreed on which walkers were most vulnerable, and which exhibited submissiveness and/or negative affect. The consensus correlations suggested that the observers agreed with one another on which walkers were most vulnerable to experience sexual victimization, as well as which walkers appeared to be easy targets. The observers did not agree on which walkers were most vulnerable to experience violent victimization, however the result approached significance. 
Given the findings from Study 1, which indicated that those who were violently victimized exhibited a vulnerable gait, it is interesting that observers were more likely to agree with one another on which walkers were most vulnerable to sexual (as opposed to violent) victimization. It is possible that different and/or additional movement cues are considered when individuals make decisions regarding one's vulnerability to sexual crime (e.g., Ritchie, 2014). However, given that several of the walkers with violent victimization experiences had also been sexually victimized, the findings that differ by type of victimization must be interpreted carefully. The collection of additional pointlight walkers is necessary in order to ensure that similar results are obtained when individuals who have been victimized both violently and sexually are isolated.

In terms of personality, the consensus correlations indicated that the observers agreed on which walkers appeared the most self-confident, but not on which walkers appeared assertive. Given that both self-confidence and assertiveness are components of Assured-Dominance in the IASR-B5, it is unclear the extent to which observers agree on the apparent dominance of others. The observers did not agree on which walkers appeared submissive (i.e., the consensus correlations for both items [timid and unaggressive] that comprise the Unassured-Submissive scale in the IASR-B5 were nonsignificant). Therefore, hypothesis one, which expected that observers would agree on which walkers were submissive, was not supported.

In relation to affect, the observers agreed on which walkers appeared strong. However, they did not reach consensus on the remaining positive affect items (alert, cheerful, determined, and happy). All the consensus correlations for the negative affect items were non-significant as well; observers did not agree on which walkers appeared 
afraid, hostile, sad, or upset. Given previous research suggesting that zero-acquaintance observers are relatively good at detecting cues of emotion from non-verbal behaviour, particularly displays of negative emotion, the results are surprising (e.g., Schneider et al., 2014).

The memory exercise that the walkers completed may have affected how strong and self-confident they appeared. Recall that the walkers were asked to observe the researcher walking in a pattern around the laboratory; they were then tasked with following the same path that the researcher had outlined for them in order to deflect their attention away from their gait movement. Because they were focused on where to walk, some of the walkers may have exhibited a stride that appeared purposeful (e.g., they may have walked with their head up as they concentrated on where to go); this type of a walk may have also been perceived as self-confident and/or strong to observers. Research is required to better understand how gait changes as a function of the situation an individual is in, or the task they are provided.

As previously noted, because it is difficult to film individuals without their knowledge, particularly in point-light conditions, the present study averted the walkers' attention away from their movement by employing a memory task; it was the intention of the researcher to capture the walkers' most natural gait. However, future research may wish to examine whether gait movement changes when individuals are less vigilant and/or do not know that they are being watched. It may then be established whether the memory task confounded the individuals' gait movement (i.e., by unintentionally eliciting a more purposeful gait). That being said, it is also possible that disagreements across observers (i.e., low consensus agreement) was due to the fact that observers simply 
felt uncomfortable providing ratings of negative traits (i.e., there may have been a social desirability bias in effect). Perhaps asking observers to "imagine themselves as a mugger/offender," similar to the directions provided by Wheeler et al. (2009), would allow one to obtain more honest ratings of negative traits (and higher levels of agreement).

\section{Accuracy}

The second hypothesis pertained to the accuracy of the observers (i.e., did the observers tend to rate those with a previous victimization history as those most vulnerable to experience victimization in the future?). Observer ratings were tallied and percentages were calculated, with the results suggesting that the majority of the observers were accurate. However, the accuracy observed from the percentage calculations appeared to be largely the result of the observers providing low ratings of vulnerability to walkers without victimization histories, and less to do with observers identifying walkers who had previous victimization experiences as those most vulnerable to future victimization.

Therefore, to further examine the accuracy of the observers, ROC analyses were conducted. The ROC results were not affected by the base rate of victims in the sample and tested the accuracy of the observers at various thresholds along the vulnerability scale. The results confirmed that the observers did not truly discriminate between previously victimized and non-victimized walkers based on the degraded point-light footage. In other words, observers did not rate the walkers with self-reported histories of victimization as more vulnerable. 
Although using a metric that is not affected by the base rate (such as the AUC that emerges from ROC analysis) is a possible option for determining observer accuracy, additional methodological measures can be taken to ensure that observers are truly distinguishing past victims as future victims based on degraded movement information. For example, future research may wish to present observers with an even number of previously victimized walkers and non-victimized walkers to ensure that measures of accuracy are not biased by the base rate of victims in the sample (i.e., such that in a sample with many more victims than non-victims, observers cannot simply rate all the walkers as highly vulnerable and obtain a high accuracy score, or vice versa).

Given that the AUC statistics did contain pooled data (i.e., the observers' ratings of vulnerability for every walker, in each model, was averaged), the results must be interpreted cautiously. Pooling multiple observers' data, while common practice when employing ROC analysis (Swets \& Pickett, 1982), can average out differences and/or disagreement among observers and result in lost information (Erkel \& Pattynama, 1998). Extreme ratings in either direction (which may be accurate) are essentially diminished when the average is taken. However, the nature of the current data limited the analyses possible. Future analyses, wherein the accuracy of each of the observers is calculated for each of the walkers that they rate, may help retain any lost information that may have resulted using the current method of pooling.

While the observers in the current sample may not have been accurate in terms of providing higher vulnerability ratings to those who had been previous victims of crime, they did provide higher ratings of vulnerability to those who exhibited more vulnerable gait cues. The fact that observers could distinguish individuals with vulnerable coded gait 
patterns from those without vulnerability cues present in their gait suggests that observers perceive subtle differences in gait movement and can use such differences to make decisions regarding vulnerability. The results further suggested that observers retain the ability to distinguish between vulnerable and non-vulnerable gait movement, even when provided substantially degraded information (i.e., 10 seconds of point-light footage). This result is consistent with previous literature (Gunns et al., 2002; Wheeler et al., 2009) and provides additional evidence suggesting that zero-acquaintance observers tend to choose individuals with vulnerable gait cues as those most vulnerable to future victimization.

Accuracy correlations were also calculated between the observers' perceptions of the walkers' personality and affect, and the walkers' self-reports of their own personality and affect. As expected in hypothesis three, self-other agreement accuracy was not obtained for any of the items. Thus, it appears that the way one perceives them self may not be how a stranger perceives them. It is unclear why the walkers' perceptions of themselves were incongruent with the way the observers perceived them. One possibility is that the walkers perceived themselves differently than how they actually are. For example, early work by Rogers (1951) argues that one's ideal self is often different from one's true self; individuals strive toward self-actualization wherein these two selves become less distinct.

Similarly, individuals often engage in self-serving biases wherein they attribute more positive states and traits to themselves: “...far from being balanced between the positive and the negative, the perception of self that most individuals hold is heavily weighted toward the positive end of the scale...individuals judge positive personality attributes to be more descriptive of themselves than of the average person..." (Kruglanski 
\& Higgins, 2003, p. 26). Indeed, a study by Lewinsohn, Mischel, Chaplin, and Barton (1980) had judges observe students engaged in a task. They then asked the judges to rate the students on their perceived personality. The students also rated their own personality features. The results revealed that the students rated themselves significantly higher on the positive traits than the observers rated them. Thus, it is possible that the walkers reported their personality and/or affect to be different than how it appeared to the observers, perhaps because they perceived themselves more positively (i.e., they reported the way they would like to be, instead of how they truly are).

On the other hand, it is possible that the observers simply had difficulty ascertaining personality and/or affect from the point-light footage. Although some prior studies showcasing degraded movement have suggested that strangers are fairly skilled at detecting traits in others (e.g., Naumann, Vazire, Rentfrow, \& Gosling, 2009), others have argued for the alternative. For example, some researchers have argued that, although observers appear to agree with one another on the type of individual a person is, they are not necessarily accurate (Levesque \& Kenny, 1993). Furthermore, given research suggesting that, “...people also tend to use their positive qualities when appraising others, thereby virtually assuring a favourable self-other comparison" (Kruglanski \& Higgins, 2003, p. 26), observers may rate walkers below average on their positive personality and affective states in an attempt to achieve a more positive self-concept (i.e., they can then downward compare themselves).

Some researchers have also argued that the malleability of the self may affect the way that an individual is perceived or appears to others (e.g., Markus \& Kunda, 1986). An individual's self-concept can change as a result of their circumstances, or the social 
environment that they find themselves in (e.g., Demo, 1992). Given the nature of the current study, it is possible that the walkers altered their persona to better adapt to the situation at hand (i.e., they were involved in meeting researchers, changing into a Vicon suit, concentrating on the memory task, etc.). Research contrasting the gait patterns of individuals involved in varying situations, particularly those which are more stressful than others, is required. Since participants were also forewarned that they would be asked about their victimization history (e.g., in recruitment and in the consent form), they may also have adjusted their self-concept based on the prime.

It should be noted that although the Victim Vulnerability Rating Questionnaire attempted to limit the questions that the observers were asked by only including facevalue measures of personality and affect (e.g., "sad," "determined," etc.), the brief, timerestricted measures may have contributed to the lack of association observed between observer ratings of walker personality and affect, and the walkers' own self-reports of their personality and affect. Certain adjectives that were not used may have more accurately reflected the constructs that the researcher aimed to measure (i.e., submissiveness, dominance, negative and positive affect). It is also possible that some observers may have experienced fatigue when asked to provide multiple ratings of vulnerability, personality and affect; some observers may have simply failed to properly assess and score each walker. However, observers were only asked to rate a randomly selected half of the videos (i.e., 14 of 28) to mitigate potential fatigue effects. Furthermore, given the low rates of missing data, attrition did not appear to be an issue in the present study. 
In addition, it is important to consider that the observers were a Canadian community sample. Thus, the results require replication using an offender sample. A community sample allowed the researcher to collect a large amount of data in a short period of time, while simultaneously increasing the generalizability of the results (i.e., the sample was arguably more representative of the typical Canadian population in terms of gender, age, and ethnicity, than a student population would have been). An apriori power analysis had estimated that a sample size of 134 would be required, and the sample obtained $(n=129)$ allowed the researcher to retain power. Nevertheless, an offender sample would ensure that the type of detection ability observed in community members generalizes more broadly to perpetrators who exploit vulnerability in others.

That being said, previous research suggests that offenders do show similar levels of ability in detecting cues of vulnerability to that of students and/or the general population (e.g., Book et al., 2013; Wheeler et al., 2009). Wheeler et al. (2009) found that student observers chose past victims, who exhibited vulnerable gait cues, as future victims. Using the same walker videos as Wheeler et al. (2009), Book et al. (2013) tested incarcerated offenders and the offender observers also chose individuals who had been previously victimized as those most vulnerable to future victimization. It may be argued that if students, who will typically have less experience in victim selection, can detect cues of vulnerability, then offenders would display a similar ability, if not be better at the task.

It is also important to consider that the personality and/or affect of the observers may have had an effect on their ratings of walker vulnerability. For example, some research suggests that certain clusters of personality traits, such as those related to 
psychopathy (Book et al., 2013; Ritchie, 2014; Wheeler et al., 2009), impacts the observers' ratings. ${ }^{36}$ Given that the current thesis was focused to a large extent on the potential reasons why individuals with victimization histories exhibit differential gait patterns, the background of the observers was not examined. Furthermore, because the observers were asked to rate approximately 14 video clips, and provide fairly comprehensive reviews for each, additional questions may have resulted in attrition. However, it would be valuable in future research to examine how walker personality and observer personality, as well as walker affect and observer affect, influences ratings of vulnerability.

The effect of observer demographic characteristics on rating accuracy was briefly examined. Prior to this thesis, Gunns et al.'s (2002) research was one of the only studies to examine the effect of observer gender on ratings of vulnerability. Therefore, a series of chi-square analyses were conducted in order to examine differences in rating accuracy for males and females. The findings were consistent with Gunns et al. (2002) in that no major effects were observed. However, additional research with equally large samples of male and female observers, as well as male and female walkers, is required before firm conclusions can be reached regarding the impact of gender on vulnerability ratings. Because the majority of the observer population was Caucasian, differences in ratings as a function of ethnicity could not be examined, but this would be another valuable line of future research.

\footnotetext{
${ }^{36}$ Some research suggests that a small percentage of the general (non-offender) population possess psychopathic traits (e.g., "subclinical psychopathy;" Levenson, Kiehl, \& Fitzpatrick, 1995; Williams et al., 2007). Given that the present sample consisted of community members, it is possible that those exhibiting more psychopathic characteristics may have displayed greater accuracy in their ratings.
} 


\section{The Effect of the Observers' Perceptions of the Walkers' Personality and Affect on Ratings of Vulnerability}

Prior research has assumed that the gait pattern observed in victims of crime may be an outward expression of their personality and/or affect, particularly submissiveness. However, the findings in Study 1 suggested that neither personality nor affect appeared to be strongly related to the likelihood of one experiencing victimization. Only dominance had a significant association with gait, indicating that individuals who self-reported dominant personality characteristics were more likely to exhibit vulnerable movement features. Given that the observers did not appear to be accurate in how they perceived the walkers' personality and affect (i.e., their judgements of the walkers' personality and affect did not correspond to how the walkers self-interpreted their own personality and affect), hypothesis four aimed to determine whether the observers' perceptions of the walkers' personality and affect (accurate or not) impacted their ratings of the walkers' vulnerability. It was expected that the observers' perceptions of the walkers' personality and affect would predict the vulnerability rating provided.

The first regression model indicated that, on average, the walkers who were rated as submissive (i.e., timid and unaggressive) were perceived to be significantly more vulnerable to violent victimization relative to others. The finding confirms hypotheses put forth in previous literature and suggests that the personality traits perceived by strangers can predict how vulnerable one appears. That being said, given that the observers were a random sample of community members, the results require replication using an offender population. However, if community members select those who appear submissive (and who exhibit vulnerable gait cues) as those most vulnerable to victimization, then it is 
likely that offenders (some of whom have heightened detection skills and/or experience in selecting vulnerable victims to exploit) would exhibit similar abilities.

Interestingly, the second regression model that was constructed suggested that, while observers perceive potential victims of violent crime to be highly submissive, they do not tend to perceive potential victims of sexual crime to be highly submissive. As previously suggested, it may be the case that observers use different and/or additional cues (over and above gait) to make judgements regarding one's vulnerability to sexual victimization. For example, perhaps the observers attended to the walkers' head movement, arm swing, or overall energy to assess their vulnerability to sexual victimization; the current sample of walkers were not rated on these traits though they have been coded for in similar research (e.g., A. Book, personal communication, May 7 , 2015). Alternatively, it is possible that submissive individuals are simply less apt to find themselves in situations that would make them vulnerable to be perpetrated against. In the majority of sexual assault cases, the victims know their perpetrator (Sinozich \& Langton, 2014). Therefore, victim qualities in sexual crime may be quite different from those found in victims of violent crime.

Future research may wish to consider investigating the type of traits (and emotions) that offenders perceive in walkers, and to examine the extent to which they use this information to make decisions regarding vulnerability. Interestingly, the walkers in Study 1 who reported dominant personality characteristics appeared to exhibit more vulnerable features in their gait. Yet, the observers in Study 2 selected individuals they perceived to be submissive as those most likely to be victimized in the future (the observers also selected those with more vulnerable gait cues as those most likely to 
experience future victimization). Thus, it is possible that the same walkers who are reporting dominant personality characteristics are being perceived by others as submissive (these individuals appear to be exhibiting more vulnerable gait cues, and observers are rating them as having a higher likelihood [i.e., as being more vulnerable] to experience future victimization).

\section{The Effect of the Walkers' own (Self-reported) Personality and Affect on Likelihood to Experience Victimization}

In an attempt to further understand the effect of personality and affect on victimization, an exploratory logistic regression analysis was conducted. The results indicated that, on average, as self-reported dominance increased, the odds of having a violent victimization history declined. Given the findings in Study 1, which suggested that Assured-Dominance was significantly related to gait, and not associated with victimization history, the present findings are intriguing. While dominant individuals may be less likely to experience victimization, it appears that they do exhibit a noticeably different (though not necessarily "vulnerable") gait pattern.

Given the inconsistent findings between dominance, submissiveness, vulnerability, gait, and victimization history, additional research is required to understand the type of individuals who become victims of crime, and what effect victimization has on personality and/or affect. For example, it may be of interest to examine the changes that take place in individuals who take self-defence classes post-assault in order to feel more confident and less fearful; indeed, there are entire classes dedicated to self-defence for women and/or for victims of assault (e.g., Can-Ryu Jiu-Jitsu Self-Defence Martial Arts). It is possible that after an individual is victimized, they work to express more 
dominance in an attempt to prevent any more future incidences of victimization.

However, while they may intend to communicate dominance to outsiders, their gait appears to betray their vulnerability.

\section{General Discussion}

In sum, the results from Study 1 supported previous findings (e.g., Wheeler et al., 2009) suggesting that a recognizably different gait pattern is observed in individuals who have experienced victimization. The findings also indicated that, contrary to expectation, walkers who reported that they were particularly dominant were more likely to exhibit vulnerable gait features. However, while both previous victimization and dominance were related to gait, there was no relationship observed between victimization history and dominance. Therefore, dominance could not explain the association between victimization history and gait.

Furthermore, the results of Study 1 suggested that individuals with violent victimization histories in particular exhibited significantly more vulnerable gait cues, whereas those with sexual victimization histories did not. The timing of one's victimization, as well as the frequency with which they had experienced victimization, appeared to have little effect on gait. However, the severity of one's victimization experience(s) did have some relation to gait. On average, individuals who had been violently victimized reported more severe victimization experiences. Recall that those with violent victimization histories were more likely to exhibit vulnerable gait cues. Individuals who reported very severe (defined subjectively) sexual victimization experiences, were also more likely to exhibit vulnerable gait cues. Therefore, the severity of one's victimization may be related to the vulnerability level of their gait. 
The findings from Study 2 indicated that zero-acquaintance observers tended to agree on which walkers were easy targets, and they were more likely to rate walkers who displayed vulnerable gait cues as those most likely to experience future victimization. Thus, it appears that individuals who exhibit the vulnerable gait pattern may be at an increased risk of experiencing victimization. Notably, however, the observers were not accurate in their ratings (i.e., they did not select previous victims as those most vulnerable to future victimization). Thus, it may be argued that the observers made judgements based on the walkers' coded gait features, and not another (unmeasured) cue that only the victimized walkers retained.

The observers in Study 2 also failed to accurately detect any personality traits or affective states of the walkers. Indeed, while walkers who reported that they were particularly dominant were more likely to exhibit vulnerable gait cues, observers selected individuals who they rated as submissive, and whom exhibited vulnerable gait cues, as those most likely to experience victimization in the future. Interestingly, walkers who were perceived to be submissive were rated as most vulnerable to violent victimization, while those perceived to be vulnerable to sexual victimization were not rated as highly submissive.

\section{Nonverbal Communication}

The current thesis aimed to determine the type of information (e.g., vulnerability) that can be obtained via specific non-verbal behaviours (such as gait). Importantly, confounds (such as age, clothing, attractiveness, etc.) were removed in the current sample of walkers, and observers were only shown a "thin slice" (i.e., a silent 10-second video clip) of an individual walking. The findings suggested that certain gait movements 
communicate vulnerability to zero-acquaintance observers. More specifically, individuals who exhibit long or short strides, a lateral, diagonal, or up/down shift movement, a gestural walk, unilateral arm/leg movements, and lifted foot movements, tend to be perceived as those most vulnerable to experience future victimization. The findings lend credence to prior research investigating gait as a nonverbal cue of vulnerability (e.g., Book et al., 2013; Wheeler et al., 2009).

However, contrary to prior research suggesting that observers are fairly skilled at judging personality traits in others from degraded information (e.g., Ambady et al., 1999; Ambady \& Rosenthal, 1992; Carli et al., 1995; Fowler et al., 2009; Richards et al., 1991; Simpson et al., 1993; Stillman et al., 2010), the observers in the current sample were not able to accurately detect any of the walkers' personality traits. In fact, while the walkers with vulnerable gait cues self-reported personality features consistent with dominance, the observers selected walkers who exhibited vulnerable gait cues and appeared submissive, as those most vulnerable to future victimization (i.e., the observers perceived vulnerable-coded walkers as having exactly the opposite personality features that the walkers reported actually having).

Recall that Simpson et al. (1993) discussed the way in which nonverbal behaviour not only signals information to others, but also inspires action (e.g., approach and interaction, or avoidance, in observers). Although the observers were inaccurate in how they perceived the walkers' personality, their perception did predict how vulnerable they rated the walkers. More specifically, higher ratings of walker submissiveness resulted in higher ratings of vulnerability by the observers. Thus, the way that individuals "read" 
each other does appear to have implications in terms of how they may interact, or how they predict others (e.g., those looking to exploit them) will interact with them.

Nonverbal behaviour appears to be important for communicating information between individuals (e.g., Argyle, Alkema, \& Gilmour, 1971; Eskritt \& Lee, 2003). However, a greater understanding of the meaning of behavioral cues and the ability of observers to detect such cues would allow for a greater understanding of human communication. Research has suggested that individuals form impressions about others almost immediately, based on very limited information (e.g., Carney, Colvin, \& Hall, 2007). Given that gait is one of the first cues that individuals have access to when making judgements about others, additional research investigating gait as a signaller of information is required. Measures of vulnerability are particularly important as individuals may be unknowingly indicating a weakness to others through their gait (as well as, possibly, through various other attributes and behaviours; Gunns et al., 2002; Sparks, 1981).

Various factors pertaining to the observers remain untested and may have contributed to the current results. For example, given that previous findings have found that individuals who exhibit psychopathic traits are more accurate in their ratings (e.g., Book et al., 2013), it is possible that the observers' own personality and/or affect impacted the conferred ratings. It is suggested that future research explore the effect of the observers' personality and affect, and even their victimization history, on accuracy (i.e., in detecting walker vulnerability, personality, and affect). Furthermore, because the current thesis was largely correlational as well as cross-sectional in design, the findings require replication before firm conclusions can be made. 


\section{A "Victim Walk?"}

Research has suggested that both situational and environmental factors, as well as the inherent attributes of individuals, contribute to perceptions of vulnerability (e.g., Beauregard et al., 2007; Myers et al., 1984); offenders appear to use a combination of "victim-centric" cues during the process of target selection. For example, Beauregard et al. (2007) provided evidence indicating that one's routine activities (e.g., commuting to work) impacts victim choice, whereas Myers et al.'s (1984) research revealed that individuals who report submissive traits tend to exhibit certain behaviours that increase their chances of experiencing victimization (and possibly repeated victimization given the high rates of victim recidivism; Kilpatrick \& Acierno, 2003).

Similar to Myers et al. (1984), the current research focused on inherent traits of individuals (i.e., personality and affect) and behavioral cues (i.e., gait movement) that could contribute to one's vulnerability to experience victimization. As noted, the literature has assumed that individuals who are submissive exhibit behaviours that are indicative of vulnerability (e.g., Myers et al., 1984); these behaviours appear to be detected by others who aim to exploit them (e.g., Wheeler et al., 2009). While the current findings did suggest that gait may be a cue for vulnerability (i.e., observers selected individuals with certain gait patterns as those most vulnerable to future victimization), individuals who reported that they had experienced victimization and exhibited vulnerable gait features did not report that they were submissive. Rather, individuals with vulnerable gait features were significantly more likely to present with personality characteristics consistent with dominance. Therefore, submissiveness does not appear to 
be expressed through one's gait. Thus, it remains unclear why certain individuals exhibit the vulnerable "victim walk" pattern.

As previously discussed, these results suggest that the "vulnerable gait pattern" itself may need to be reconsidered. If dominant individuals are those that exhibit vulnerable gait cues, then it may not be a "vulnerable" gait at all, but rather a "dominant gait pattern." Perhaps observers are actually selecting the more difficult individuals to victimize. Given previous literature on the topic, which suggests that offenders (psychopathic offenders in particular) are proficient at selecting "easy targets," the concept seems unlikely. Nevertheless, due to the inconsistent findings, the possibility may need to be explored.

On the other hand, perhaps dominant individuals simply exhibit a different (though not necessarily "vulnerable") gait pattern, which is noticeable and detected by others. Previous research (e.g., Grayson \& Stein, 1981) has noted that observers select gait patterns that appear non-synchronous or "different" than the others, as those most vulnerable to victimization (p. 74). It is possible that observers are not truly detecting vulnerability from gait, but simply detecting a difference and rating it as significantly more different. Dominant individuals in the present sample may have been scored as having gait features that were vulnerable, when in reality they simply exhibited a more salient walking pattern and/or produced more exaggerated movements. More finegrained analyses with a larger sample of walkers would likely confirm whether observers are selecting truly dominant individuals with prior victimization experiences and vulnerable gait features, as those most likely to experience victim recidivism. 
The differential findings may also be partially attributable to the potential priming effect of the victimization history questionnaire. Although the surveys that the walkers received were randomized (e.g., some walkers completed the IASR-B5 first, others completed the Victimization History Questionnaire Extended or the PANAS-X first), it is possible that some walkers were primed to think about their victimization history prior to filling out items related to their personality and affect; this may have impacted the answers they provided. Walkers were also informed that they would be asked about their victimization history in the study invite as well as in the informed consent form. Thus, some walkers may have entered the study already thinking about their victimization experience(s), and this may have affected how they rated their personality and affect.

\section{Easy Prey: An Evolutionary Perspective}

A secondary aim of the current thesis was to advance evolutionary theory by better understanding the cognitive appraisal and detection ability of individuals. The thesis results indicated that certain individuals (i.e., those who are dominant and those who have been violently victimized in their past) exhibit gait features that others do not. These gait features appear to elicit particular impressions in others (e.g., strangers observing those who exhibit vulnerable gait features agree that they are "easy targets"). Therefore, gait may provide socially useful information to observers (i.e., certain features signal vulnerability).

The way in which gait movement communicates information to others may be understood through the concept of evolutionary fitness. Research has shown that the ability to perform and decode nonverbal behaviour is important for survival and reproduction within the animal kingdom (e.g., Darwin, 1869). For example, the 
perception of physical health in others may have consequences for mate choice. Indeed, 'survival of the fittest' is based on the premise that the strongest, smartest, and most environmentally adaptable members of a species will survive and reproduce; whereas the process of natural selection will eliminate those who are vulnerable (e.g., Darwin, 1869, Gunns et al., 2002; Schaller, 2008).

Although it is unclear whether the walkers in the present study were (unintentionally) signalling their vulnerability to others from their gait movement, certain individuals did exhibit remarkably different features relative to others. Should one adhere to the argument that biological movement is non-sporadic (Ritchie, 2014) and constrained by one's emotions and intentions (i.e., consistent with the KSD principle; Gunns et al., 2002; Runeson \& Frykholm, 1983), then it would appear that the walkers who were exhibiting the noticeably different gait pattern were communicating a specific message (through their gait).

The ecological approach to perception argues that one's level of vulnerability is specified in the social stimulus array and recognizable to others because vulnerability originally had consequences for prey selection (Gunns et al., 2002; McArthur \& Baron, 1983; Montepare \& Zebrowitz-McArthur, 1988). Indeed, prior research has provided evidence suggesting that individuals can detect submissiveness (which may be related to vulnerability) via "thin slice" information (e.g., submissive women appear to gesture more with their hands and feet; Richards. et al., 1991). Given that observers in the current study appeared to discriminate between vulnerable and non-vulnerable gait movement (i.e., they were more likely to rate those with vulnerable gait cues as those most likely to experience future victimization), it may be argued that nonverbal cues of vulnerability are 
evident to strangers. Although evolutionary theory is difficult to test directly, the current results are consistent with, and provide some evidence that supports it.

\section{Kinematic Point-Light Display}

The current research aimed to advance the literature regarding gait as a cue of vulnerability by eliminating walker confounds. The observers made decisions regarding vulnerability to victimization based on approximately 10 -seconds worth of degraded point-light footage. The findings indicated that certain gait features are indeed perceived as more vulnerable than others, even when extraneous features (e.g., clothing, sex, weight, attractiveness, etc.) have been removed. However, contrary to previous research (e.g., Book et al., 2013; Wheeler et al., 2009), the observers did not select individuals with past victimization histories as those most likely to experience future victimization. ${ }^{37}$

The differential results may have been a consequence of the point-light display technology. Because only biological movement was observable from the recordings (i.e., gait was isolated from any other cues that observers could use to make decisions from such as age, attractiveness, clothing, sex, etc.), the raters could only make decisions regarding vulnerability from movement. Ratings of vulnerability in previous studies (e.g., Wheeler et al., 2009) may have been based on gait movement cues in addition to other cues (e.g., clothing). Therefore, results that indicated that prior victims of crime may be at risk of experiencing victim recidivism, may have occurred because observers were making decisions based on a myriad of features that suggested that someone had been a prior victim of crime. Observers in the present sample were clearly selecting those with a

\footnotetext{
${ }^{37}$ Recall that although individuals who experienced victimization were significantly more likely to exhibit vulnerable gait cues, several individuals who did not have a history of victimization also exhibited vulnerable gait cues. Therefore, accuracy calculations were conducted to examine whether observers selected those with prior victimization histories as vulnerable, and/or if they selected those with vulnerable gaits.
} 
certain gait pattern as those most vulnerable (as movement was the only cue available), regardless of their apparent victimization history.

As noted, while walkers who reported that they were particularly dominant tended to exhibit vulnerable gait cues, observers selected individuals who appeared submissive, and whom exhibited vulnerable gait cues, as those most likely to experience victimization in the future. It is possible that the walkers were attempting to appear more dominant than they were in reality, and the observers detected their "leaked" submissiveness. Indeed, the KSD principle maintains that an individual's "dispositions" (i.e., anatomical makeup, as well as emotions and intentions) constrain and determine their movement pattern (Gunns et al., 2002; Runeson \& Frykholm, 1983). One's movement thus provides genuine information (e.g., regarding their personality, emotions, intentions, etc.). Similarly, Ekman and Friesen (1969a) argue that nonverbal behaviour(s) may "escape[s] efforts to deceive" by "leaking" withheld information (p. 52). Thus, even "hidden" properties related to personality and emotion are likely to be present in the kinematic pattern and detectable from point-light images (Runeson \& Frykholm, 1983).

On the other hand, it is possible that the observers were simply assuming that those they rated as vulnerable must be submissive and they rated them as such without considering that dominant individuals may actually exhibit different gait features. It is possible that individuals rely on heuristics or stereotypical beliefs regarding the "type" of person most likely to experience victimization. Indeed, the results also suggested that the walkers who were perceived to be submissive were rated as most vulnerable to violent victimization, while those perceived to be vulnerable to sexual victimization were not rated as highly submissive. The findings indicated that observers may use different cues 
to select for different types of crime, or that they do not associate submissiveness with sexual victimization (i.e., submissiveness is not a salient personality cue for vulnerability to sexual victimization).

As previously mentioned, one of the limitations to using point-light display is that individuals are aware that they are being filmed. Some research has suggested that individuals may alter (e.g., exaggerate or constrain) their body movements when they believe that they are being watched. For example, Johnston et al. (2004) filmed the same cohort of walkers under two different sets of instructions. First, the walkers were filmed after they had been instructed to walk as naturally as possible (i.e., "normal" condition). The participants were then asked to imagine themselves walking in a popular inner-city park, and they were filmed again (i.e., "park" condition). Spontaneous differences in walking behaviour were observed; when participants imagined themselves walking in the park they displayed greater arm swing, they walked with longer strides, and they exhibited more energy, compared to when they walked in the normal condition. Observers rated the walkers in the park condition as more difficult to attack compared to those in the normal condition. Thus, it has been suggested that gait patterns change when individuals believe that they are being watched.

That being said, Johnston et al.'s (2004) results may be better explained by the perceived safety of the imagined park and/or by participant confidence in their perceived safety, and not necessarily because the park is a public space where they would be observed by others: "Creating an experimental condition that increased the salience of personal safety resulted in spontaneous changes to walking style... The walking styles of women in the less safe (park) condition more closely resembled that of the prototypical 
low-vulnerability walker...than did those of the same walkers in the normal walking condition" (p. 533-534). Indeed, the current thesis found that individuals who had been previously victimized exhibited vulnerable gait cues, despite evidence suggesting that increased levels of vigilance on the part of the walkers extinguishes vulnerability. In other words, consistent with Gunns et al. (2002), the walkers in Study 1 were aware that they were being filmed throughout the duration of the study, and yet certain individuals still exhibited the "victim walk" pattern.

Given that certain individuals exhibit considerably different gait patterns, relative to others, and that such movement cues appear to impact one's vulnerability (regardless of self-reported personality and/or affective characteristics), it is important to establish ways that individuals can reduce their outward vulnerability. It is necessary to qualify that the study of characteristics that are theorized to increase one's vulnerability to experience victimization does not excuse the offender from his/her exclusive responsibility. A comprehensive study of both the characteristics that make one vulnerable to an attack, as well as the elements that result in an offender perpetrating a crime, are necessary in order to fully understand the interactions involved in victimization. Similarly, techniques or tactics aimed at reducing one's vulnerability does not supersede interventions instituted to prevent offenders from perpetrating the act in the first place.

Researchers have suggested that individuals desiring proactive prevention strategies enroll in self-defence classes (e.g., Johnston et al., 2004). They argue that these classes may help eliminate obvious cues of nonverbal vulnerability by making individuals aware of vulnerable body language and/or by increasing their feelings of 
safety. Johnston et al. (2004) explored whether or not feelings of safety impact walking style. A sample of females $(n=5)$ completed a self-defence course (Johnston et al., 2004). Pre- and post-course gait patterns were recorded and observers rated them on their vulnerability to victimization (the independent sample of raters included 25 males and 26 females). Surprisingly, the results revealed no differences in walking style, nor were there differences in ratings of vulnerability across sessions. It is possible that the tactics learned in the self-defence course simply did not impact one's feeling of safety, and therefore did not affect the participants' movement cues. The small sample of walkers may also have affected the significance of the findings. Additional research examining the effect of feelings of safety on nonverbal behaviour, as well as the influence of self-defence courses on unconscious movement, is required.

Another experiment examined differences in gait style following individualized body awareness training sessions in a sample of five female walkers who had been rated as "easy to attack" (Johnston et al., 2004). All five participants completed individualized training sessions where a researcher explained the features of a vulnerable gait pattern to them and taught them to move without displaying these features. A sample of observers rated the vulnerability of the walkers. Significant differences were observed:

"Differences in vulnerability between sessions were revealed and could be accounted for by changes in walking-style features" (p. 514). The walkers were rated as less vulnerable (i.e., observers scored them as harder to attack) after training compared to before training. A follow-up study revealed that differences in gait style persisted for at least one month following training. 
Overall, the preliminary findings suggest that gait patterns may respond adaptively when in a potentially dangerous situation, and that it may be possible to train individuals to walk with different features than they normally would (in day-to-day life), thereby mitigating their overall vulnerability. Replication of the results is required, particularly with larger samples. However, empirically-driven intervention/prevention strategies aimed at targeting nonverbal cues of vulnerability are an encouraging step toward reducing victimization. Applied research pertaining to gait movement and nonverbal cuing is necessary, and remains a virtually undiscovered subject area.

\section{Conclusion}

Victimology is in its infancy, but it has become a crucial component in crime reduction and prevention strategies. The current research aimed to expand the extremely novel literature regarding gait as a victim selection cue, and was one of the first to investigate both observer choice and victim cueing using kinematic point-light display. Most notably, observers agreed on which individuals appeared to be easy targets, and they selected individuals who exhibited vulnerable gait cues as those most likely to experience victimization using only the point-light stimuli. Therefore, there is additional evidence suggesting that individuals can in fact infer vulnerability from gait.

Examination of the victim selection process is important theoretically in terms of advancing evolutionary understanding and cognitive appraisal processes. A greater realization of both observer detection ability and the translation of internal states to external behaviour has been captured, thereby providing an indication as to why certain individuals may be repeatedly selected for victimization. More practically, understanding how non-criminal populations use body language cues to detect vulnerability is likely to 
provide some understanding as to how predatory offenders (who seek vulnerability) would be able to use them as well. Greater consideration of nonverbal cues of vulnerability may lead to the design and implementation of proactive and targeted strategies (e.g., body awareness training) that may help to prevent future incidences of victimization (Johnston et al., 2004). 


\section{References}

Abbey, A. (2002). Alcohol-related sexual assault: A common problem among college students. Journal of Studies on Alcohol, Supplement, 14, 118-128.

Abbey, A., \& McAuslan, P. (2004). A longitudinal examination of male college students' perpetration of sexual assault. Journal of Consulting and Clinical Psychology, 72(5), 747-756.

Acton, G. S., \& Revelle, W. (2002). Interpersonal personality measures show circumplex structure based on new psychometric criteria. Journal of Personality Assessment, 79(3), 446-471.

Alison, L. J., \& Stein, K. L. (2001). Vicious circles: Accounts of stranger sexual assault reflect abusive variants of conventional interactions. The Journal of Forensic Psychiatry, 12(3), 515-538.

Allison, P. D., \& Oaks, T. (2002). Missing data: Quantitative applications in the social sciences. British Journal of Mathematical and Statistical Psychology, 55(1), 193196.

Almagor, M., \& Ben-Porath, Y. (1989). The two-factor model of self-reported mood: A cross-cultural replication. Journal of Personality Assessment, 53, 10-21.

Ambady, N., Conner, B., \& Hallahan, M. (1999). Accuracy of judgements of sexual orientation from thin slices of behavior. Journal of Personality and Social Psychology, 77(3), 538-547.

Ambady, N., \& Rosenthal, R. (1992). Thin slices of expressive behavior as predictors of interpersonal consequences: A meta-analysis. Psychological Bulletin, 111(2), 256-274.

Amir, M. (1971). Patterns in forcible rape. Chicago, IL: University of Chicago Press. 
Anderson, C., \& Kilduff, G. J. (2009). Why do dominant personalities attain influence in face-to-face groups? The competence-signaling effects of trait dominance. Journal of Personality and Social Psychology, 96(2), 491-503. doi: $10.1037 / \mathrm{a} 0014201$

Asch, S. E. (1946). Forming impressions of personality. The Journal of Abnormal and Social Psychology, 41(3), 258-290. doi: 10.1037/h0055756

Baron, R. M., \& Kenny, D. A. (1986). The moderator-mediator variable distinction in social psychological research: Conceptual, strategic and statistical considerations. Journal of Personality and Social Psychology, 51, 1173-1182.

Beardsworth, T., \& Buckner, T. (1981). The ability to recognize oneself from a video recording of one's movements without seeing one's body. Bulletin of the Psychonomic Society, 18, 19-22.

Beauregard, E., \& Leclerc, B. (2007). An application of the rational choice approach to the offending process of sex offenders: A closer look at the decision-making. Sex Abuse, 19, 115-133. doi: 10.1007/s11194-007-9043-6

Beauregard, E., Rossmo, K. D., \& Proulx, J. (2007). A descriptive model of the hunting process of serial sex offenders: A rational choice perspective. Journal of Family Violence, 22, 449-463. doi: 10.1007/s10896-007-9101-3

Bender, R., \& Lange, S. (2001). Adjusting for multiple testing — when and how? Journal of Clinical Epidemiology, 54(4), 343-349.

Bennell, C., Mugford, R., Ellingwood, H., \& Woodhams, J. (2013). Linking crimes using behavioural clues: Current levels of linking accuracy and strategies for moving 
forward. Journal of Investigative Psychology and Offender Profiling, 11(1), 2956.

Birdwhistell, R. L. (1970). Kinesics and context: Essays on body motion communication. Philadelphia, PA: University of Pennsylvania Press.

Blais, J., Solodukhin, E., \& Forth, A. E. (2014). A meta-analysis exploring the relationship between psychopathy and instrumental versus reactive violence. Criminal Justice and Behavior, 41(7), 797-821. doi: 10.1177/0093854813519629.

Book, A., Costello, K., \& Camilleri, J. A. (2013). Psychopathy and victim selection: The use of gait as a cue to vulnerability. Journal of Interpersonal Violence, 28(11), 2368-2383. doi: $10.1177 / 0886260512475315$

Breitenbecher, K. H. (2000). Sexual assault on college campuses: Is an ounce of prevention enough? Applied and Preventive Psychology, 9(1), 23-52.

Brinke, L., \& Porter, S. (in press). Friend or foe? The role of intuition in interpersonal trustworthiness and vulnerability assessments. The Psychology of Intuition.

Carli, L. L., LaFleur, S. J., \& Loeber, C. C. (1995). Nonverbal behavior, gender, and influence. Journal of Personality and Social Psychology, 68(6), 1030-1041.

Carney, D. R., Colvin, C. R., \& Hall, J. A. (2007). A thin slice perspective on the accuracy of first impressions. Journal of Research in Personality, 41(5), 10541072.

Cattell, R. B. (1946). The description and measurement of personality. New York, NY: World Book.

Cohen, J. (1992). A power primer. Psychological Bulletin, 112(1), 155-159. 
Cohen, L. E., \& Cantor, D. (1981). Residential burglary in the United States: Life-style and demographic factors associated with the probability of victimization. Journal of Research in Crime and Delinquency, 18(1), 113-127. doi:

$10.1177 / 002242788101800107$

Cohen, J., \& Cohen, P. (1983). Applied multiple regression/correlation analysis for the behavioral sciences. Hillsdale, NJ: Lawrence Erlbaum Associates, Inc.

Cornish, D. B., \& Clark, R. V. (1986). The reasoning criminal: Rational choice perspectives on offending. New York, NY: Springer-Verlag.

Costa, P. T. Jr., \& McCrae, R. R. (1985). The NEO Personality Inventory manual. Odessa, FL: Psychological Assessment Resources.

Crystal, D. (1969). Prosodic systems and intonation in English. Cambridge, London: Cambridge University Press.

Cutting, J. E. (1977). Recognizing friends by their walk: Gait perception without familiarity cues. Bulletin of the Psychonomic Society, 9, 353-356.

Darwin, C. (1869). On the origin of species by means of natural selection, or the preservation of favoured races in the struggle for life ( $\left.5^{\text {th }} \mathrm{ed}.\right)$, London, England: John Murray.

Demo, D. H. (1992). The self-concept over time: Research issues and directions. Annual Review of Sociology, 18, 303-326. doi: 10.2307/2083456

Diener, E., Larsen, R. J., Levine, S., \& Emmons, R. A. (1985). Intensity and frequency: Dimensions underlying positive and negative affect. Journal of Personality and Social Psychology, 48, 1253-1265. 
Dittrich, W. H., Troscianko, T., Lea, S. E., \& Morgan, D. (1996). Perception of emotion from dynamic point-light displays represented in dance. Perception, 25(6), 727738.

Ehninger, D., Gronbeck, B., McKerrow, R., \& Monroe, A. (1986). Principles and types of speech communication (10 ${ }^{\text {th }}$ ed.). Glenville, IL: Scott Foresman and Co.

Eibl-Eibesfeldt, I. (1989). Human ethology. New York, NY: Aldine de Gruyter.

Ekman, P. (1964). Body position, facial expression, and verbal behavior during interviews. Journal of Abnormal and Social Psychology, 68(3), 295-301.

Ekman, P. (1972). Universals and cultural differences in facial expressions of emotion. In J. Cole (Ed.), Nebraska symposium on motivation, 1971 (pp. 207-283). Lincoln, NE: University of Nebraska Press.

Ekman, P., \& Friesen, W. V. (1965). Personality, pathology, affect, and nonverbal behavior. Paper presented at the Western Psychological Association Convention, Hawaii.

Ekman, P., \& Friesen, W. V. (1967). Head and body cues in the judgement of emotion: A reformulation. Perceptual and Motor Skills, 24, 711-724.

Ekman, P., \& Friesen, W. V. (1968). Nonverbal behavior in psychotherapy research. In J. Shlien (Ed.), Research in psychotherapy: Vol III (pp. 179-216). Washington, DC: American Psychological Association.

Ekman, P., \& Friesen, W. V. (1969a). The repertoire of nonverbal behavior: Categories, origins, usage, and coding. Semiotica, 49-98.

Ekman, P., \& Friesen, W. V. (1969b). Nonverbal leakage and clues to deception. Psychiatry, 32(1), 88-106. 
Van Erkel, A. R., \& Pattynama, P. M. (1998). Receiver operating characteristic (ROC) analysis: Basic principles and applications in radiology. European Journal of Radiology, 27(2), 88-94.

Fanschawe, J. H., \& Fitzgibbon, C. D. (1993). Factors influencing the hunting success of an African wild dog pack. Animal Behavior, 45, 479-490.

Faul, F., Erdfelder, E., Buchner, A., \& Lang, A. G. (2009). Statistical power analyses using $\mathrm{G}^{*}$ Power 3.1: Tests for correlation and regression analyses. Behavior Research Methods, 41, 1149-1160.

Field, A. (2009). Discovering statistics using SPSS (3 ${ }^{\text {rd }}$ ed.). Thousand Oaks, CA: SAGE Publications Ltd.

Filipas, H. H., \& Ullman, S. E. (2006). Child sexual abuse, coping responses, self-blame, posttraumatic stress disorder, and adult sexual re-victimization. Journal of Interpersonal Violence, 21(5), 652-672.

Fisher, B. S., Sloan, J. J., Cullen, F. T., \& Lu, C. (1998). Crime in the ivory tower: The level and sources of student victimization. Criminology, 36(3), 671-710.

Fiske, A. P. (1991). Structures of social life: The four elementary forms of human relations: Communal sharing, authority ranking, equality matching, market pricing. New York, NY: Free Press.

Fowler, K. A., Lilienfeld, S. O., \& Patrick, C. J. (2009). Detecting psychopathy from thin slices of behavior. Psychological Assessment, 21(1), 68-78. doi: $10.1037 / \mathrm{a} 0014938$

Frijda, N. (2010). Impulsive action and motivation. Biological Psychology, 84, 570-579. doi: 10.1016/j.biopsych.2010.01.005 
Gough, H. (1975). Manual for the California Psychological Inventory. Palo Alto, CA: Consulting Psychologists Press.

Graham, G. H., Unruh, J., \& Jennings, P. (1991). The impact of nonverbal communication in organizations: A survey of perceptions. International Journal of Business Communication, 28, 45-62. doi: 10.1177/002194369102800104

Grayson, B., \& Stein, M. I. (1981). Attracting assault: Victims' nonverbal cues. Journal of Communication, 68-75.

Gunns, R. E., Johnston, L., \& Hudson, S. M. (2002). Victim selection and kinematics: A point light investigation of vulnerability to attack. Journal of Nonverbal Behavior, $26(3), 129-158$.

Gurtman, M. B. (2009). Exploring personality with the Interpersonal Circumplex. Social and Personality Psychology Compass, 3, 1-19. doi: 10.1111/j.17519004.2009.00172.x

Hall, J. A., LeBeau, L. S., \& Coats, E. J. (2005). Nonverbal behavior and the vertical dimension of social relations: A meta-analysis. Psychological Bulletin, 131(6), 898-924. doi: 10.1037/0033-2909.131.6.898

Hampson, S. E., \& Goldberg, L. R. (2006). A first large cohort study of personality trait stability over the 40 years between elementary school and midlife. Journal of Personality and Social Psychology, 91(4), 763-779. doi: 10.1037/00223514.91.4.763

Hare, R. D. (2001). Psychopaths and their nature: Some implications for understanding human predatory violence. In A. Raine \& J. Sanmartin (Eds.), Prospective studies of crime and delinquency (pp. 225-236). Boston, MA: Kluwer-Nijhoff. 
Hare, R. D. (2003). The Hare Psychopathy Checklist-Revised ( $2^{\text {nd }}$ ed). Toronto, Ontario: Multi-Health Systems.

Hare, R. D., \& Neumann, C. S. (2006). The PCL-R assessment of psychopathy. Handbook of psychopathy, 58-88.

Hare, R. D., \& Neumann, C. S. (2008). Psychopathy as a clinical and empirical construct. Annual Review of Clinical Psychology, 4, 217-246. doi:10.1146/annurev.clinpsy.3.022806.091452

Hayes, A. F. (2013). Introduction to mediation, moderation, and conditional process analysis: A regression-based approach. New York, NY: Guilford Press.

Hegstrom, T. (1979). Message impact: What percentage is nonverbal? The Western Journal of Speech Communication, 43, 134-142.

Henley, N. M. (1977). Body politics: Power, sex, and nonverbal communication. Englewood Cliff, NJ: Prentice-Hall.

Hill, H., \& Pollick, F. E. (2000). Exaggerating temporal differences enhances recognition of individuals from point light displays. Psychological Science, 11(3), 223-228.

Hoenkamp, E. (1978). Perceptual cues that determine the labeling of human gait. Journal of Human Movement Studies, 4, 59-69.

Hogan, R. (1986). Manual for the Hogan Personality Inventory. Minneapolis, MN: National Computer Systems.

Hough, M. (1987). Offenders' choice of target: Findings from victim surveys. Journal of Quantitative Criminology, 3(4), 355-369.

Ikeda, H., \& Watanabe, K. (2009). Anger and happiness are linked differently to the explicit detection of biological motion. Perception, 38, 1002-1011. 
James, L. R., \& Brett, J. M. (1984). Mediators, moderators and tests for mediation. Journal of Applied Psychology, 69, 307-321.

Johansson, G. (1973). Visual perception of biological motion and a model for its analysis. Perception and Psychophysics, 14, 201-211.

Johnston, L., Hudson, S. M., Richardson, M. J., Gunns, R. E., \& Garner, M. (2004). Changing kinematics as a means of reducing vulnerability to physical attack. Journal of Applied Social Psychology, 34(3), 514-537.

Joslin, C. (2010). Motion capture. Retrieved from http://mocap.csit.carleton.ca/index.php?Section=Overview\&Item=Default\&Page= Default

Judd, C. M., \& Kenny, D. A. (1981). Process analysis: Estimating mediation in treatment evaluations. Evaluation Review, 5, 602-619.

Judge, T. A., Bono, J. E., Ilies, R., \& Gerhardt, M. W. (2002). Personality and leadership: A qualitative and quantitative review. Journal of Applied Psychology, 87(4), 765780. doi: $10.1037 / / / 0021-9010.87 .4 .765$

Karg, M., Kuhnlenz, K., \& Buss, M. (2010). Recognition of affect based on gait patterns. IEEE Transactions on Systems, Man, and Cybernetics: Part B: Cybernetics, 40(4), 1050-1061.

Kern, J. M. (1982). Predicting the impact of assertive, empathic-assertive, and nonassertive behavior: The assertiveness of the assertee. Behavior Therapy, 13(4), 486-498. doi: 10.1016/S0005-7894(82)80011-7

Kilpatrick, D. G., \& Acierno, R. (2003). Mental health needs of crime victims: Epidemiology and outcomes. Journal of Traumatic Stress, 16(2), 119-132. 
Kilpatrick, D. G., Acierno, R., Resnick, H. S., Saunders, B. E., \& Best, C. L. (1997). A two year longitudinal analysis of the relationship between violent assault and alcohol and drug use in women. Journal of Consulting and Clinical Psychology, 65(5), 834-847.

Kilpatrick, D. G., Saunders, B. E., Amick-McMullan, A., Best, C. L., Veronen, L. J., \& Resnick, H. S. (1989). Victim and crime factors associated with the development of crime-related post-traumatic stress disorder. Behavior Therapy, 20, 199-2014.

Knapp, M., Hall, J., \& Horgan, T. (2013). Nonverbal communication in human interaction. Cengage Learning.

Krauss, R. M., Chen, Y., \& Chawla, P. (1996). Nonverbal behavior and nonverbal communication: What do conversational hand gestures tell us? In M. Zanna (Ed.), Advances in experimental social psychology (pp. 389-450). San Diego, CA: Academic Press.

Kruger, J. (1999). Lake Wobegon be gone! The" below-average effect" and the egocentric nature of comparative ability judgments. Journal of Personality and Social Psychology, 77(2), 221-232.

Kruglanski, A. W., \& Higgins, E. T. (2003). Social psychology: A general reader. New York, NY: Taylor \& Francis Books Inc.

Kumar, R., \& Indrayan, A. (2011). Receiver operating characteristic (ROC) curve for medical researchers. Indian Pediatrics, 48(4), 277-287.

Larsen, R. J., \& Buss, D. M. (2008). Personality psychology. McGraw Hill.

Lauritsen, J. L., \& Quinet, K. F. D. (1995). Repeat victimization among adolescents and young adults. Journal of Quantitative Criminology, 11(2), 143-166. 
Levenson, M. R., Kiehl, K. A., \& Fitzpatrick, C. M. (1995). Assessing psychopathic attributes in a noninstitutionalized population. Journal of Personality and Social Psychology, 68(1), 151-158.

Levesque, M. J., \& Kenny, D. A. (1993). Accuracy of behavioral predictions at zero acquaintance: A social relations analysis. Journal of Personality and Social Psychology, 65(6), 1178-1187.

Lewinsohn, P. M., Mischel, W., Chaplin, W., \& Barton, R. (1980). Social competence and depression: The role of illusory self-perceptions. Journal of Abnormal Psychology, 89(2), 203-212.

Locke, K. D. (2006). Interpersonal circumplex measures. In S. Stack (Ed.), Differentiating normal and abnormal personality ( $2^{\text {nd }}$ ed., pp. 383-400). New York, NY: Springer Publishing Co.

Lord, R. G., de Vader, C. L., \& Alliger, G. M. (1986). A meta-analysis of the relation between personality traits and leadership perceptions: An application of validity generalization procedures. Journal of Applied Psychology, 71(3), 402-410. doi: 10.1037/0021-9010.71.3.402

Mahmut, M. K., Menictas, C., Stevenson, R. J., \& Homewood, J. (2011). Validating the factor structure of the Self-Report Psychopathy Scale in a community sample. Psychological Assessment, 23(3), 670.

Mandal, F. B. (2014). Nonverbal communication in humans. Journal of Human Behavior in the Social Environment, 24, 417-421. doi: 10.1080/10911359.2013.831288 
Markey, P. M., \& Markey, C. N. (2009). A brief assessment of the Interpersonal Circumplex: The IPIP-IPC. Assessment, 16(4), 352-361. doi: $10.1177 / 1073191103940382$

Markus, H., \& Kunda, Z. (1986). Stability and malleability of the self-concept. Journal of Personality and Social Psychology, 51(4), 858-866.

Matsumoto, D., Keltner, D., Shiota, M. N., O’Sullivan, M., \& Frank, M. (2008). Facial expressions of emotion. In M. Lewis, J. M. Haviland-Jones, \& L. Feldman Barrett (Eds.), Handbook of emotions ( ${ }^{\text {rd }}$ ed., pp. 211-234). New York, NY: The Guilford Press.

Mazur, A. (1985). A biosocial model of status in face-to-face primate groups. Social Forces, 64(2), 377-402.

McArthur, L. Z., \& Baron, R. M. (1983). Toward an ecological theory of social perception. Psychological Review, 90, 215-238.

McCrae, R. R., \& Costa, P. T. Jr. (1989). The structure of interpersonal traits: Wiggins' Circumplex and the Five-Factor Model. Journal of Personality and Social Psychology, 56(4), 586-595.

Mehrabian, A. (1972). Nonverbal communication. Chicago, IL: Aldine-Atherton.

Mehrabian, A., \& Ferris, S. R. (1967). Inference of attitudes from nonverbal communication in two channels. Journal of Consulting Psychology, 31(3), 248252. doi: $10.1037 / \mathrm{h} 0024648$

Mehrabian, A., \& Wiener, M. (1967). Decoding of inconsistent communications. Journal of Personality and Social Psychology, 6, 109-114. doi: 10.1037/h0024532 
Miethe, T. D., \& Meier, R. F. (1990). Opportunity, choice, and criminal victimization: A test of a theoretical model. Journal of Research in Crime and Delinquency, 27(3), 243-266.

Montepare, J. M., Goldstein, S. B., \& Clausen, A. (1987). The identification of emotions from gait information. Journal of Nonverbal Behavior, 11(1), 33-42.

Montepare, J. M., \& Zebrowitz-McArthur, L. (1988). Impressions of people created by age-related qualities of their gaits. Journal of Personality and Social Psychology, $55(4), 547-556$.

Montepare, J. M., \& Zebrowitz, L. A. (1993). A cross-cultural comparison of impressions created by age-related variations in gait. Journal of Nonverbal Behavior, 17, 5568.

Murzynski, J., \& Degelman, D. (1996). Body language of women and judgements of vulnerability to sexual assault. Journal of Applied Social Psychology, 26(18), $1617-1626$.

Myers, M. B., Templer, D. I., \& Brown, R. (1984). Coping ability in women who become victims of rape. Journal of Consulting and Clinical Psychology, 52, 73-78. doi: 10.1037/0022-006X.52.1.73

Neumann, C. S., Kosson, D. S., \& Salekin, R. T. (2007). Exploratory and confirmatory factor analysis of the psychopathy construct: Methodological and conceptual issue. In H. Herve' \& J. C. Yuille (Eds.), The psychopath: Theory, research, and practice (pp. 79-104). Mahwah, NJ: Erlbaum. 
Naumann, L. P., Vazire, S., Rentfrow, P. J., \& Gosling, S. D. (2009). Personality judgments based on physical appearance. Personality and Social Psychology Bulletin, 35(12), 1661-1671.

Neumann, C. S., Vitacco, M. J., Hare, R. D., \& Wupperman, P. (2005). Reconstruing the "reconstruction" of psychopathy: A comment on Cooke, Michie, Hart, and Clark. Journal of Personality Disorders, 19(6), 624-640.

Norris, F. H., \& Kaniasty, K. (1994). Psychological distress following criminal victimization in the general population: Cross-sectional, longitudinal, and prospective analyses. Journal of Consulting and Clinical Psychology, 62(1), 111123.

Paulhus, D. L., Neumann, C. S., \& Hare, R. D. (in press). Self-report psychopathy scale: Version III. Toronto, Ontario: Multi-Health Systems.

Pollick, F. E., Lestou, V., Ryu, J., \& Cho, S. B. (2002). Estimating the efficiency of recognizing gender and affect from biological motion. Vision research, 42(20), 2345-2355.

Porter, L. E., \& Alison, L. J. (2004). Behavioural coherence in violent group activity: An interpersonal model of sexually violent gang behaviour. Aggressive Behavior, 30, 449-468. doi: 10.1002/ab.20047

Rogers, C. R. (1951). Client-centered therapy: Its current practice, implications and theory. Boston, MA: Houghton Mifflin.

Royal Canadian Mounted Police. (2014, October). Ask an expert: Walk this way, forensic gait analyst identifies suspects by their gait. Gazette, 76(3). Retrieved from http://www.rcmp-grc.gc.ca/gazette/vol76no3/expert-eng.htm 
Richards, L. (1991). A theoretical analysis of nonverbal communication and victim selection for sexual assault. Clothing and Textiles Research Journal, 9(4), 55-64. doi: $10.1177 / 0887302 X 9100900408$

Richards, L., Rollerson, B., \& Phillips, J. (1991). Perceptions of submissiveness: Implications for victimization. The Journal of Psychology, 125(4), 407-411.

Ritchie, M. (2014). Perceptions of victim vulnerability: The role of personality and psychopathy (Unpublished undergraduate honour's thesis). Carleton University, Ottawa Ontario.

Runeson, S., \& Frykholm, G. (1983). Kinematic specification of dynamics as an informational basis for person-and-action perception: Expectation, gender recognition, and deceptive intention. Journal of Experimental Psychology: General, 112, 585-615.

Sable, M. R., Danis, F., Mauzy, D. L., \& Gallagher, S. K. (2006). Barriers to reporting sexual assault for women and men: Perspectives of college students. Journal of American College Health, 55(3), 157-162.

Sakaguchi, K., \& Hasegawa, T. (2006). Person perception through gait information and target choice for sexual advances: Comparison of likely targets in experiments and real life. Journal of Nonverbal Behavior, 30, 63-85. doi: 10.1007/s10919006-0006-2

Sapir, E. (1949). The unconscious patterning of behavior in society. In D. Mandelbaum (Ed.), Selected writings of Edward Sapir in language, culture and personality (pp. 533-563). Berkeley, CA: University of California Press. 
Schaller, M. (2008). Evolutionary bases of first impressions. In N. Ambady \& J. J. Skowronski (Eds.), First impressions (pp. 1-15). New York, NY: Guilford Press.

Schneider, S., Christensen, A., Haubinger, F. B., \& Fallgatter, A. J. (2014). Show me how you walk and I tell you how you feel-A functional near-infrared spectroscopy study on emotion perception based on human gait. NeuroImage, 85 , 380-390.

Selkin, J. (1975). Rape: When to fight back. Psychology Today, 8(8), 71-76.

Simpson, J. A., Gangestad, S. W., \& Biek, M. (1993). Personality and nonverbal social behaviour: An ethological perspective of relationship initiation. Journal of Experimental Social Psychology, 29, 434-461.

Sinozich, S., \& Langton, L. (2014). Rape and sexual assault victimization among collegeage females, 1995-2013. (Research Report No. NCJ 248471). Retrieved from Bureau of Justice Statistics website: http://www.bjs.gov/content/pub/pdf/rsavcaf9513.pdf

Smyth, M. M., \& Pendleton, L. R. (1990). Space and movement in working memory. The Quarterly Journal of Experimental Psychology, 42(2), 291-304.

Snook, B., \& Cullen, R. M. (2009). Bounded rationality and criminal investigations: Has tunnel vision been wrongfully convicted. In D. K. Rossmo (Ed.), Criminal investigative failures (pp. 71-98). Boca Raton, FL: Taylor and Francis Group.

Sparks, R. (1981). Multiple victimization: Evidence, theory, and future research. Journal of Criminal Law and Criminology, 72, 762-778.

Stevens, D. J. (1994). Predatory rapists and victim selection techniques. The Social Science Journal, 31(4), 421-433. 
Stewart, J., \& D’ Angelo, G. (1988). Together: Communicating interpersonally (3 ${ }^{\text {rd }}$ ed.). New York, NY: Newberry Award Records, Inc.

Stillman, T. F., Maner, J. K., \& Baumeister, R. F. (2010). A thin slice of violence: Distinguishing violent from nonviolent sex offenders at a glance. Evolution and Human Behaviour, 31(4), 298-303.

Studenmund, A. H., \& Cassidy, H. J. (1987). Using econometrics: A practical guide. Boston, MA: Little, Brown.

Sullivan, H. S. (1953). The interpersonal theory of psychiatry. New York: Norton.

Swets, J. A. (1988). Measuring the accuracy of diagnostic systems. Science, 240(4857), $1285-1293$.

Swets, J. A., \& Pickett, R. M. (1982). Evaluation of diagnostic systems: Methods from signal detection theory. New York, NY: Academic Press.

Tabachnick, B. G., \& Fidell, L. S. (2013). Using Multivariate Statistics (6 ${ }^{\text {th }}$ ed.). Boston, MA: Pearson.

Tapscott, J. L., Hancock, M., \& Hoaken, P. N. S. (2012). Severity and frequency of reactive and instrumental violent offending: Divergent validity of subtypes of violence in an adult forensic sample. Criminal Justice and Behavior, 39(2), 202219. doi: $10.1177 / 0093854811429647$

Thompson, E. R. (2007). Development and validation of an internationally reliable shortform of the Positive and Negative Affect Schedule (PANAS). Journal of CrossCultural Psychology, 38(2), 227-242. doi: 10.1177/0022022106297301 
Tomasello, M., Call, J., Nagell, K., Olguin, R., \& Carpenter, M. (1994). The learning and use of gestural signals by young chimpanzees: A trans-generational study. Primates, 35(2), 137-154.

Trapnell, P. D., \& Wiggins, J. S. (1990). Extension of the Interpersonal Adjective Scales to include the Big Five dimensions of personality. Journal of Personality and Social Psychology, 59(4), 781-790.

Truman, J. L. (2011). Criminal victimization, 2010. National Crime Victimization Survey, U.S. Department of Justice, Office of Justice Programs, Bureau of Justice Statistics (NCJ 235508), 1-20.

Van Damme, R., \& Van Dooren, J. M. (1999). Absolute versus per unit body length speed of prey as an estimator of vulnerability to predation. Animal Behaviour, 57, 347-352.

Van den Stock, J., Righart, R., \& de Gelder, B. (2007). Body expressions influence recognition of emotions in the face and voice. Emotion, 7(3), 487-494. doi: $10.1037 / 1528-3542.7 .3 .487$

Van Selst, M., \& Jolicoeur, P. (1994). A solution to the effect of sample size on outlier estimation. The Quarterly Journal of Experimental Psychology, 47, 631-650.

Vitacco, M. J., Neumann, C. S., \& Jackson, R. L. (2005). Testing of a four-factor model of psychopathy and its association with gender, ethnicity, intelligence and violence. Journal of Consulting and Clinical Psychology, 73, 466-476. doi:10.1037/0022-006X.73.3.466

Wang, Q., Zhou, X., \& Lu, X. (2013). The injury feigning displays of the Red-crowned crane. The Wilson Journal of Ornithology, 125(2), 415-417. 
Wasserman, E., \& Ellis, C. A. (2007). Impact of crime on victims. National Victim Assistance Academy, Track 1, Foundation-Level Training, V1-1-V1-16.

Watson, D., \& Clark, L. A. (1994). The PANAS-X: Manual for the Positive and Negative Affect Schedule-Expanded form. Arnes, IA: The University of Iowa.

Watson, D., Clark, L. A., \& Tellegen, A. (1988). Development and validation of brief measures of positive and negative affect: The PANAS scales. Journal of Personality and Social Psychology, 54(6), 1063-1070.

Wheeler, S., Book, A., \& Costello, K. (2009). Psychopathic traits and perceptions of victim vulnerability. Criminal Justice and Behavior, 36(6), 635-648. doi: $10.1177 / 0093854809333958$

Wiggins, J. S. (1979). A psychological taxonomy of trait-descriptive terms: The interpersonal domain. Journal of Personality and Social Psychology, 37, 395-412.

Wiggins, J. S. (1995). Interpersonal Adjective Scales professional manual. Odessa, FL: Psychological Assessment Resources.

Wiggins, J. S. (2003). Paradigms of personality assessment. New York, NY: Guilford Press.

Wiggins, J. S., Trapnell, P., \& Phillips, N. (1988). Psychometric and geometric characteristics of the revised Interpersonal Adjective Scales (IAS-R). Multivariate Behavioral Research, 23, 517-530

Williams, K. M., Paulhus, D. L., \& Hare, R. D. (2007). Capturing the four-factor structure of psychopathy in college students via self-report. Journal of Personality Assessment, 88, 205-219.

Wolff, W. (1943). The expression of personality. New York, NY: Harper. 
Wright, R., Logie, R. H., \& Decker, S. H. (1995). Criminal expertise and offender decision making: An experimental study of the target selection process in residential burglary. Journal of Research in Crime and Delinquency, 32(1), 39-53.

Yeagley, E., Morling, B., \& Nelson, M. (2007). Nonverbal zero-acquaintance accuracy of self-esteem, social dominance orientation, and satisfaction with life. Journal of Research in Personality, 41, 1099-1106. doi: 10.1016/j.jrp.2006.12.002 
Appendices 


\section{Appendix A}

Study 1: Movement Code

1. STRIDE LENGTH: Distance measured by a step.

Short Medium

Long

2. TYPE OF WEIGHT SHIFT: The shift that occurs when transferring weight from one foot to the other while walking. Movement usually starts at the pelvis.

Primarily lateral: Weight shifted from side to side

Three-dimensional: Pelvis operates in a spiral and achieves a three-dimensional quality

Primarily up/down: Weight shift causes a bounce because body goes up and down

Primarily forward/back: A sagittal

\section{TYPE OF WALK:}

Postural: Movement activates the whole body

Gestural: Movement activates only a part of the body

Non-specific: Other

\section{BODY MOVEMENT:}

Unilateral: One side of body or one limb used

Contralateral: two sides of body move into counterpoint - right arm, left leg then left arm, right leg

\section{FEET:}

Swung __ Lifted 
Appendix B

Mass Testing Section 1: Victimization History Questionnaire

Walker Pre-selection Survey: Victimization History

Section 1: The following questions relate to your history of criminal victimization.

Please complete the questions as honestly and as carefully as possible by checking the appropriate box. You are not required to answer any question that you do not feel comfortable answering, and may stop or skip any of the questions at any time. If you do not wish to answer a particular question, simply check the box entitled "Check this box if you do not want to provide an answer for this question."

1. Have you ever experienced violent victimization (e.g., mugging, assault)?

$\neg$ Yes

$\square$ No

$\square$ Check this box if you do not want to provide an answer for this question

2. Have you ever experienced sexual victimization (e.g., rape, stalking)?

$\square$ Yes

$\square$ No

$\square$ Check this box if you do not want to provide an answer for this question

3. Have you ever experienced non-violent victimization (e.g., break and enter, car theft)

$\square$ Yes

$\square$ No

$\square$ Check this box if you do not want to provide an answer for this question 
Appendix C

Study 1 \& Study 2: Demographic Questionnaire

\section{Demographics}

Please answer the following questions by checking the appropriate box or writing your answer in the space provided.

1. What is your age? (years)

2. What is your gender?

3. What is your ethnic background?

$\square$ Caucasian/White

$\square$ African Canadian/Black

$\square$ First Nations/Aboriginal Canadian/Native Canadian

$\square$ Asian

$\square$ East Indian

$\square$ Hispanic/Latino

$\square$ Middle Eastern

$\square$ Other (please specify): 
Appendix D

Study 1: Victimization History Questionnaire Extended

\section{Victimization History}

The following questions relate to your history of criminal victimization. Please complete the questions as honestly and as carefully as possible by checking the appropriate box. Before beginning, please consult the glossary below. If you have any additional questions, please do not hesitate to ask the researcher. You are not required to answer any question that you do not feel comfortable answering, and may stop or skip any of the questions at any time. If you do not wish to answer a particular question, simply check the box entitled "Check this box if you do not want to provide an answer for this question."

\section{Victimization History Glossary}

Sexual victimization: Sexual abuse, any non-consensual sexual activity (e.g., sexual assault, rape, etc.), threat of unwanted sexual contact, sexual trafficking, sexual defamation (e.g., sending of nude photos), sexual coercion, etc.

Violent victimization: Physical bullying, in addition to any of the following crimes: Robbery, mugging, assault, physical abuse, neglect, harassment, battery (e.g., intimate partner violence), kidnapping, gang violence, threats, etc.

If answering any of these questions made you feel distressed or uncomfortable, and you would like to speak to someone about your thoughts, please feel free to contact Carleton University Health and Counseling Services at 613-520-6674 (http://www.carleton.ca/health/) or the Distress Centre of Ottawa and Region at 613-238-3311 (http://www.dcottawa.on.ca). 
1. Have you ever experienced violent victimization?

$\neg$ Yes

$\square$ No

$\square$ Check this box if you do not want to provide an answer for this question

2. If no, please move to question \#13. If yes, when did this occur? Note. If you have been violently victimized multiple times, please refer to the most serious experience for this question.

$\neg$ Very recently (i.e., within the last few weeks)

$\square$ Recently (i.e., within the last 6 months)

$\square$ Quite a while ago (i.e., 1-2 years ago)

$\square$ Long ago (i.e., $2-5$ years ago)

$\square$ Very long ago (i.e., $5+$ years ago)

$\square$ Check this box if you do not want to provide an answer for this question

3. How many times have you experienced violent victimization?

$\square$ Once

$\square$ Twice

$\square$ 3-5 times

$\square 5+$ times

$\square$ Check this box if you do not want to provide an answer for this question

4. Did your violent victimization experience(s) ever cause you distress?

$\square$ None (i.e., no distress experienced)

$\square$ Minimal (e.g., initial stress, which later dissipated; some symptoms present, etc.)

$\square$ Moderate (e.g., experienced certain long-lasting difficulties; notified authorities, etc.)

$\square$ Severe (e.g., experienced persistent, interfering symptoms; received counseling; notified authorities, etc.)

$\square$ Check this box if you do not want to provide an answer for this question

If answering any of these questions made you feel distressed or uncomfortable, and you would like to speak to someone about your thoughts, please feel free to contact Carleton University Health and Counseling Services at 613-520-6674 (http://www.carleton.ca/health/) or the Distress Centre of Ottawa and Region at 613-238-3311 (http://www.dcottawa.on.ca). 
5. Did you ever think that you were in danger or that you might be seriously injured?

$\square$ Yes

$\square$ No

$\square$ Check this box if you do not want to provide an answer for this question

6. Were you ever seriously injured?

$\square$ Yes

$\square$ No

$\square$ Check this box if you do not want to provide an answer for this question

7. When you were violently victimized, did you ever feel very afraid, helpless, or horrified?

$\square$ Yes

$\square$ No

$\square$ Check this box if you do not want to provide an answer for this question

8. On average, how long have you been bothered by your violent victimization experience(s)?

$\square$ Not at all

$\square 1$ week

$\square$ 2-3 weeks

$\square$ A month or more

$\square$ Check this box if you do not want to provide an answer for this question

9. To what extent did your violent victimization experience(s) bother you emotionally?

$\square \quad$ Not at all

$\square$ A little

$\square$ Somewhat

$\square$ Much

$\square$ Very much

$\square$ Check this box if you do not want to provide an answer for this question

If answering any of these questions made you feel distressed or uncomfortable, and you would like to speak to someone about your thoughts, please feel free to contact Carleton University Health and Counseling Services at 613-520-6674 (http://www.carleton.ca/health/) or the Distress Centre of Ottawa and Region at 613-238-3311 (http://www.dcottawa.on.ca). 
10. If possible, please rank (by circling the number below), the emotional severity of your victimization experience(s) on the 5-point scale. Note. If you have been violently victimized multiple times, please refer to the most serious experience for this question.

1

Not Severe

Severe
3
4

\section{5}

11. If possible, please rank (by circling the number below), the psychological severity of your victimization experience(s) on the 5-point scale. Note. If you have been violently victimized multiple times, please refer to the most serious experience for this question.

1

Not Severe

Severe
Extremely

12. If possible, please rank (by circling the number below), the physical severity of your victimization experience(s) on the 5-point scale. If you have been violently victimized multiple times, please refer to the most serious experience for this question.

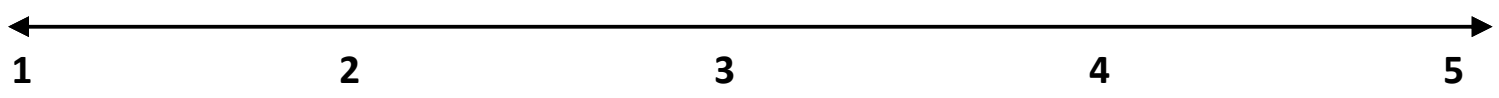

Not Severe

Extremely

Severe

If answering any of these questions made you feel distressed or uncomfortable, and you would like to speak to someone about your thoughts, please feel free to contact Carleton University Health and Counseling Services at 613-520-6674 (http://www.carleton.ca/health/) or the Distress Centre of Ottawa and Region at 613-238-3311 (http://www.dcottawa.on.ca). 
13. Have you ever experienced sexual victimization?

$\neg$ Yes

$\square$ No

$\square$ Check this box if you do not want to provide an answer for this question

14. If no, please move to the next section. If yes, when did this occur? Note. If you have been sexually victimized multiple times, please refer to the most serious experience for this question.

Very recently (i.e., within the last few weeks)

$\square$ Recently (i.e., within the last 6 months)

$\square$ Quite a while ago (i.e., 1-2 years ago)

$\square$ Long ago (i.e., 2-5 years ago)

$\square$ Very long ago (i.e., $5+$ years ago)

$\square$ Check this box if you do not want to provide an answer for this question

15. How many times have you experienced sexual victimization?

$\square$ Once

$\square$ Twice

$\square$ 3-5 times

$\square 5+$ times

$\square$ Check this box if you do not want to provide an answer for this question

16. Did your sexual victimization experience(s) ever cause you distress?

$\neg$ None (i.e., no distress experienced)

$\square$ Minimal (e.g., initial stress, which later dissipated; some symptoms present, etc.)

$\square$ Moderate (e.g., experienced certain long-lasting difficulties; notified authorities, etc.)

$\square$ Severe (e.g., experienced persistent, interfering symptoms; received counseling; notified authorities, etc.)

$\square$ Check this box if you do not want to provide an answer for this question

If answering any of these questions made you feel distressed or uncomfortable, and you would like to speak to someone about your thoughts, please feel free to contact Carleton University Health and Counseling Services at 613-520-6674 (http://www.carleton.ca/health/) or the Distress Centre of Ottawa and Region at 613-238-3311 (http://www.dcottawa.on.ca). 
17. Did you ever think that you were in danger or that you might be seriously injured?

$\square$ Yes

$\square$ No

$\square$ Check this box if you do not want to provide an answer for this question

18. Were you ever seriously injured?

$\square$ Yes

$\square$ No

$\square$ Check this box if you do not want to provide an answer for this question

19. When you were sexually victimized, did you ever feel very afraid, helpless, or horrified?

$\square$ Yes

$\square$ No

$\square$ Check this box if you do not want to provide an answer for this question

20. On average, how long have you been bothered by your sexual victimization experience(s)?

$\square$ Not at all

$\square 1$ week

$\square \quad$ 2-3 weeks

$\square$ A month or more

$\square$ Check this box if you do not want to provide an answer for this question

21. To what extent did your sexual victimization experience(s) bother you emotionally?

$\square$ Not at all

$\square$ A little

$\square$ Somewhat

$\square$ Much

$\square$ Very much

$\square$ Check this box if you do not want to provide an answer for this question

If answering any of these questions made you feel distressed or uncomfortable, and you would like to speak to someone about your thoughts, please feel free to contact Carleton University Health and Counseling Services at 613-520-6674 (http://www.carleton.ca/health/) or the Distress Centre of Ottawa and Region at 613-238-3311 (http://www.dcottawa.on.ca). 
22. If possible, please rank (by circling the number below), the emotional severity of your victimization experience(s) on the 5-point scale. Note. If you have been sexually victimized multiple times, please refer to the most serious experience for this question.

1

Not Severe

Severe
3
4

Extremely

23. If possible, please rank (by circling the number below), the psychological severity of your victimization experience(s) on the 5-point scale. Note. If you have been sexually victimized multiple times, please refer to the most serious experience for this question.

1

Not Severe

Severe
Extremely

24. If possible, please rank (by circling the number below), the physical severity of your victimization experience(s) on the 5-point scale. Note. If you have been sexually victimized multiple times, please refer to the most serious experience for this question.

1

Not Severe

Severe
3
4

\section{5}


Study 1: Recruitment Email

Appendix E

Participant Recruitment

Name of Researchers: Brittany Blaskovits \& Dr. Craig Bennell

Principal Researchers Contact Information: brittanyblaskovits@cmail.carleton.ca

Title of Study: A Kinematic Point-Light Investigation of Memory and Movement

Brief Description: You are being contacted because you completed the Mass Testing Psychology Survey in the Fall of 2014. The study you are being recruited for aims to investigate the degree to which one's personality, emotional experiences, and past life stressors, impact body-based memory (e.g., motor memory). Should you choose to participate in the current study, you will be asked to complete a short demographic questionnaire, as well as three self-report questionnaires. The questionnaires will measure various personality traits, affective states, and ask for information on your victimization history. You will also be asked to wear a fitted black suit and walk around a room while you are being recorded. The specialized cameras that are used allow you to appear as a moving lighted skeleton. At the end of the study you will be debriefed about its purpose. The study will take approximately one hour to complete and you will receive $1 \%$ credit for your participation, which can be allocated to one of your introductory psychology courses.

Is this a Web-based Study? No.

Is this a Two-part Study? No.

Length of Study: Approximately one hour.

Selection Criteria: You are being invited to participate in the current study as a result of your Fall 2014 Mass Testing scores.

Ethics Clearance Number (REB\#): This study has received clearance by the Carleton University Ethics Committee for Psychological Research (Research Ethics Approval \#15057).

Sign-up: If you are interested in participating, please sign up at: https://carleton.sonasystems.com/Default.aspx?ReturnUrl=/

If you have any questions or concerns, please do not hesitate to contact the principal researcher. Thank you for your consideration! 


\section{Appendix F}

\section{Study 1: Consent Form (deception)}

The purpose of an informed consent is to ensure that you understand the purpose of the study and the nature of your involvement. The informed consent must provide sufficient information such that you have the opportunity to determine whether you wish to participate in the study. Please take the time to read this information carefully.

\section{A Kinematic Point-Light Investigation of Memory and Movement}

\section{Research Personnel:}

Brittany Blaskovits

Department of Psychology, Graduate Student, Carleton University

brittanyblaskovits@cmail.carleton.ca

Craig Bennell

Department of Psychology, Faculty, Carleton University

craig.bennell@carleton.ca

\section{Purpose:}

Research indicates that memory does not only reside in the brain, but rather, is largely body-based (e.g., muscle memory; Smyth \& Pendleton, 1990). However, it is possible that one's personality, emotional experiences, and past life stressors, impact recall. In conducting this study, we hope to gain an understanding of the effects on motor memory.

\section{Study Procedure:}

You will be asked to complete a short demographic questionnaire, as well as three selfreport questionnaires. These questionnaires will measure various personality traits, affective states, and ask for information on your victimization history. You will also be asked to wear a fitted black suit and walk around a room while you are being recorded. The suit, which comes in a variety of sizes, may be applied over snug clothing; baggy sweaters and shirts may need to be removed. The suit is easy to get on and can be changed into with or without assistance. A private change room is available right across the hall from the studio. The suit is equipped with light reflective markers, which will be recorded by specialized cameras. The cameras will record you walking around the laboratory space and completing a simple memory exercise. Participants may be asked to tie up long hair so that it does not interfere with the camera capture; hair elastics are provided. In the recording you will appear as a moving lighted skeleton. At the end of the study you will be debriefed about its purpose. The study will take approximately one hour to complete and you will receive $1 \%$ bonus for your participation, which can be allocated to one of your introductory psychology courses. 


\section{Time Required:}

The study will take approximately 1 hour to complete.

\section{Remuneration:}

You will receive 1\% credit towards your PSYC 1001, PSYC 1002, PSYC 2001, or PSYC 2002 grade as compensation for your participation.

\section{Right to Withdraw:}

You have the right to withdraw from this study at any point, without penalty. If you choose to withdraw before the end of the study, simply let the researcher know that you have chosen to withdraw. You will still receive your $1 \%$ credit toward your psychology course.

\section{Potential Risks:}

The following risks are associated with participating in the current study: You will be asked about your victimization experiences, which may cause some discomfort. If you anticipate that reporting about your victimization experiences may cause you distress, you may choose not to participate. In the event that you do choose to participate and feel uncomfortable at any point during the study, please be reminded that your participation is entirely voluntary. You may choose not to answer any question in the surveys and you may opt not to partake in the point-light display walking. You may withdraw at any time if you no longer wish to participate. You will not experience any negative consequences if you decide to withdraw from the study, or decide not to answer any specific questions.

Please be aware that the current study is part 1 of a multi-part study. The edited (10second) walking clips produced in the present study will be shown to a separate sample of undergraduate students who will rate them on various perceived characteristics. The "walkers" will only appear to the observers as lighted skeletons whom lack any identifying features. The sample of observers will not be told who created the pointlight walkers, when they were created, or any background history of the walkers. The observers will never have access to any of your survey responses; they will only view the brief, degraded point-light figures.

\section{Confidentiality:}

All data collected will be kept confidential. The questionnaire responses will be coded in such a way that participants cannot be identified (i.e., each participant will be randomly assigned a number that cannot be linked back to them). Gait will be recorded using point-light display technology. The technology is such that only light-reflected markers appear on the recording (please see Figure 1 below). Therefore, participants are unrecognizable from observation of the video clips. 


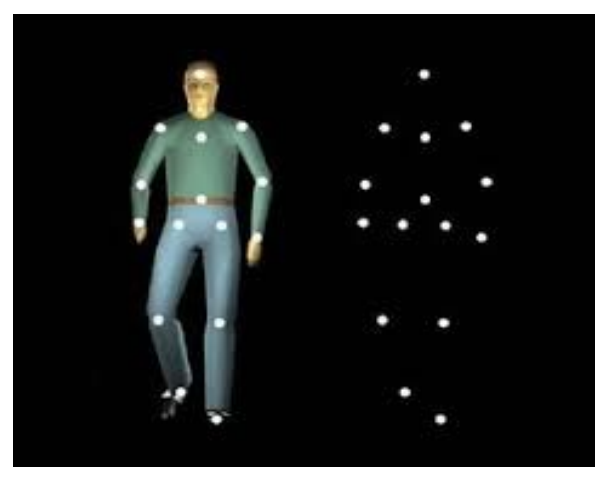

Figure 1. Typical point-light display walker

The videos will be edited down to a 10-second clip by a trained technician, who has signed a confidentiality agreement. The data will then be stored in a statistical database and kept on a secure computer in Dr. Bennell's research laboratory. The only individuals who will be able to access these files are Dr. Bennell and his research assistants and/or graduate students, who have also signed confidentiality forms ensuring that they will not reveal the content of file information. The data will be kept in locked cabinets or in password protected computer files for five years, as required by the University, before being shredded or deleted.

Again, please be aware that the current study is part 1 of a multi-part study. The brief walking clips produced in the current study will be shown to a separate sample of undergraduate students who will rate them on various perceived characteristics. The "walkers" will only appear to the observers as lighted skeletons whom lack any identifying features. The sample of observers will not be told who created the pointlight walkers, when they were created, or any background history of the walkers.

\section{Contact for Additional Study Information:}

If you have any questions or desire further information with respect to this study, you may contact:

Brittany Blaskovits at brittanyblaskovits@cmail.carleton.ca, or at 613-520-2600 ext. 1728

or

Dr. Craig Bennell at craig.bennell@carleton.ca, or at 613-520-2600 ext. 1769

This study has received clearance by the Carleton University Ethics Committee for Psychological Research (Research Ethics Approval \#15-057).

If you have any ethical concerns regarding this study, please contact:

Carleton University Ethics Committee for Psychological Research Chair: Dr. Shelley Brown

Phone: 613-520-2600, ext. 1505 
Email: shelley.brown@carleton.ca

If you have any other concerns regarding this study, please contact:

Psychology Departmental Chair: Dr. Joanna Pozzulo

Phone: 613-520-2600 ext. 1412

Email: joanna.pozzulo@carleton.ca

\section{Consent:}

Your participation in this study is entirely voluntary and you may refuse to participate or withdraw from the study at any time.

I have read the above form and hereby consent to continue participating in this study. I am aware that the data collected in this study will be kept strictly confidential and anonymous. My signature indicates that I understand the above and wish to participate in this study.

Participant's name

(print):

Participant's signature:

Researcher's name

(print):

Researcher's signature:

Date:

Your data may be used in future studies conducted by colleagues (e.g., to replicate the study with a different observer sample or to replicate the study with animated avatars [i.e., avatars are human-like characters which can be applied over the point-light figures, and then altered to have different clothing, hairstyles, etc]). However, should your data be used in the future, all personal information and/or identifiers will be removed.

All researchers, research assistants, and/or technicians, have signed confidentiality agreements ensuring the security and anonymity of your information. Your recorded walk and survey responses are numerically coded immediately upon their completion and cannot be linked back to you.

I have read the above form and hereby consent to continue participating in this study. I am aware that the data collected in this study may be used in future studies, for research publications and/or for teaching purposes. I am aware that all data will be kept strictly confidential and anonymous. My signature indicates that I understand the above and wish to participate in this study. 
Participant's name (print):

Participant's signature:

Researcher's name

(print):

Researcher's signature:

Date: 


\section{Appendix G}

Study 1: Instructions

\section{Instructions}

The current study contains two parts: In part 1, you will be asked to wear a black suit. The suit may be applied over snug clothing; baggy sweaters and shirts may need to be removed. The suit is easy to get on and can be changed into with or without assistance. Please let the researcher know if you would like assistance. A private change room is available right across the hall from the studio. Light-reflective markers will be attached to the suit using Velcro-type material. You will be asked to walk around the laboratory space as naturally as possible and complete a simple memory exercise, while being recorded. Once the research team has captured a satisfactory gait sequence, you will be invited to remove the black suit.

In part 2, you will be provided with a package, which asks you to complete a short demographic questionnaire, a survey regarding your victimization experiences, a personality inventory, and a questionnaire that inquires about your affect (i.e., emotions). Please read the instructions at the beginning of each new questionnaire carefully. Complete the questions to the best of your ability. You are not required to answer any question that you do not feel comfortable answering, and may stop or skip any of the questions at any time without risk of penalty (i.e., without losing the $1 \%$ study credit). You will then be provided with a debriefing form, which will include additional information about the study.

The research team will guide you through each step of the study. If you have any questions, please feel free to consult the principal researcher. Your generosity and willingness to participate in this research is greatly appreciated. 
Appendix H

Study 1: Debriefing Form (reveals deception)

\section{Debriefing}

\section{Walk This Way: A Kinematic Point-Light Investigation of Victim Vulnerability}

We would like to thank you for participating in this research. Your time and effort are greatly appreciated. This debriefing information is designed to help you understand the nature of this research.

\section{What are we trying to learn in this research?}

You were told that the present study was aimed at investigating the effect of life stressors, personality, and affect, on motor memory. However, the study's true aim was to gain a better understanding of whether individuals with certain personality traits, affective states, and victimization experiences, walk differently than others.

Some researchers have suggested that there may be a relationship between gait pattern (e.g., stride length [distance between steps], foot movement [lifted steps, swinging steps], weight shift [transferring weight from one foot to another], etc.) and vulnerability to victimization (e.g., Book, Costello, and Camilleri, 2013). For example, Wheeler, Book, and Costello (2009) found that undergraduate observers were able to detect vulnerability in others from short observations of gait. The present study is interested in further examining the potential link between gait and vulnerability. In addition, the study hopes to reveal whether or not various personality traits (e.g., dominance), and/or affective states (e.g., fear) can translate into walking behaviour, and are noticeable to observers; this is why you were asked about your personality features and emotional state in the surveys.

The current research required that: a) participants walked in the same direction (for at least a portion of the recording), b) that a useable gait sequence could be captured, and c) that participants walked as naturally as possible. Therefore, you were told to follow a straight line so that a useable gait sequence could be captured, and you completed a faux memory task so that you were not primed to walk differently than you normally would; it was also employed in an attempt to distract you from your walking behaviour so that your most natural gait movement could be captured.

\section{Why is this important to psychologists or the general public?}

In determining the nonverbal cues that may make some people more vulnerable to victimization, we can begin to develop intervention strategies that may help to prevent future incidences of victimization (Ritchie, 2014). 


\section{How will my data be used?}

The current study is part 1 of a multi-part study. The edited (10-second) walking clips produced in the current study will be shown to a separate sample of undergraduate students who will rate them on various characteristics such as their perceived personality (e.g., confidence), perceived affect (e.g., happiness), and their vulnerability to victimization (sexual and violent). The "walkers" will only appear to the observers as lighted skeletons whom lack any identifying features. The sample of observers will not be told who created the point-light walkers, when they were created, or any background history of the walkers. The observers will never have access to any of the survey responses; they will only view the brief, degraded point-light figures.

Your data may be also be used in future studies conducted by colleagues (e.g., to replicate the study with a different observer sample or to replicate the study with animated avatars [i.e., avatars are human-like characters which can be applied over the point-light figures, and then altered to have different clothing, hairstyles, etc]). However, should your data be used in the future, all personal information and/or identifiers will be removed.

Please be reminded that regardless of the findings of the research, you are not at blame for your history, and are in no way responsible for any victimization you may have experienced. The relationship between gait and victimization is unclear; there is absolutely no causal evidence to date which suggests that gait can cause, or lead to, one's victimization. The purpose of the present study is to examine whether or not gait has any influence on vulnerability, as it is possible that there is no relationship. The study is measuring other potential factors (i.e., personality and affect) that may better explain the differential gait styles exhibited by individuals. Given the unclear relationship between gait and victimization, individual feedback (e.g., related to the style of one's walk) cannot be provided.

All researchers, research assistants, and/or technicians, have signed confidentiality agreements ensuring the security and anonymity of your information. Your recorded walk and survey responses are numerically coded immediately upon their completion and cannot be linked back to you. Remember that your participation is entirely voluntary. You may choose not to consent to the release of your data. You will not experience any negative consequences if you decide to withdraw from the study, or decide not to release your study data.

\section{How can I learn more?}

If you are interested in learning more about perceptions and cues of victim vulnerability please refer to the following sources:

Book, A., Costello, K., \& Camilleri, J. A. (2013). Psychopathy and victim selection: The use of gait as a cue to vulnerability. Journal of Interpersonal Violence, 28(11), 23682383. doi: $10.1177 / 0886260512475315$ 
Wheeler, S., Book, A., \& Costello, K. (2009). Psychopathic traits and perceptions of victim vulnerability. Criminal Justice and Behavior, 36(6), 635-648. doi:

10.1177/0093854809333958

\section{What if I have questions later?}

If you wish to discuss this research further, please feel free to contact:

Brittany Blaskovits by email: brittanyblaskovits@cmail.carleton.ca

and/or

Dr. Craig Bennell by email: craig.bennell@carleton.ca

If you have any ethical concerns regarding this study, please contact:

Carleton University Ethics Committee for Psychological Research Chair: Dr. Shelley

Brown

Phone: 613-520-2600, ext. 1505

Email: shelley.brown@carleton.ca

If you have any other concerns regarding this study, please contact:

Psychology Departmental Chair: Dr. Joanna Pozzulo

Phone: 613-520-2600 ext. 1412

Email: joanna.pozzulo@carleton.ca

\section{What can I do if I experience discomfort or distress after participating in this study?}

Sometimes, filling out psychological measures on our past experiences or emotions can cause us distress or discomfort. Your generosity and willingness to participate in this research is greatly appreciated, however it is not the intent of the researchers to cause you any feelings of distress. If participating in this study made you feel distressed or uncomfortable, and you would like to speak to someone about your thoughts, please feel free to contact:

- Carleton University Health and Counseling Services, located in the Carleton Technology and Training Centre (across from the parking garage), at 613-5206674 (http://www.carleton.ca/health/).

- The Distress Centre of Ottawa and Region at 613-238-3311 (http://www.dcottawa.on.ca)

- The Ottawa Rape Crisis Centre at 613-562-2333 (http://www.orcc.net/)

- The Sexual Assault Support Centre of Ottawa at 613-234-2266 (http://sascottawa.com/default.aspx)

Thank you for taking the time to participate in this study!

Your participation is greatly appreciated! 
Appendix I

Study 1: Consent Form (primary)

The purpose of an informed consent is to ensure that you understand the purpose of the study and the nature of your involvement. Please take the time to read this information carefully.

\section{Walk This Way: A Kinematic Point-Light Investigation of Victim Vulnerability}

\section{Research Personnel:}

Brittany Blaskovits

Department of Psychology, Graduate Student, Carleton University

brittanyblaskovits@cmail.carleton.ca

Craig Bennell

Department of Psychology, Faculty, Carleton University

craig.bennell@carleton.ca

\section{Study Procedure:}

You have been asked to complete a short demographic questionnaire, as well as three self-report questionnaires. The questionnaires measured various personality traits, affective states, and asked for information regarding your victimization history. You were also asked to wear a fitted black suit and walk around a room. The suit was equipped with light reflective markers, which have been recorded by specialized cameras. You appear as a moving lighted skeleton in the recording.

\section{Purpose:}

You were told that the present study was aimed at investigating the effect of life stressors, personality, and affect, on motor memory. However, the study's true aim was to gain a better understanding of whether individuals with certain personality traits, affective states, and victimization experiences, walk differently than others. The research required that: a) participants walked in the same direction (for at least a portion of the recording), b) that a useable gait sequence could be captured, and c) that participants walked as naturally as possible. Therefore, you were told to follow a straight line so that a useable gait sequence could be captured, and you completed a faux memory task so that you were not primed to walk differently than you normally would; it was also employed in an attempt to distract you from your walking behaviour so that your most natural gait movement could be captured. 


\section{Remuneration:}

You will receive 1\% credit towards your PSYC 1001, PSYC 1002, PSYC 2001, or PSYC 2002 grade as compensation for your participation.

\section{Right to Withdraw:}

Given that you are now aware of the true purpose of the study, you have the right to withdraw your data (i.e., your video recording and questionnaire answers), without penalty. If you choose to withdraw, simply let the researcher know that you have chosen to withdraw and your data will be destroyed. You will still receive your $1 \%$ credit toward your psychology course.

\section{Potential Risks:}

The following risks are associated with sharing your collected information with the research team: The current study is part 1 of a multi-part study. The edited (10-second) walking clips produced in the current study will be shown to a separate sample of undergraduate students who will rate them on various characteristics such as their perceived personality (e.g., confidence), perceived affect (e.g., happiness), and their vulnerability to victimization (sexual and violent). Again, the "walkers" will only appear to the observers as lighted skeletons whom lack any identifying features. The sample of observers will not be told who created the point-light walkers, when they were created, or any background history of the walkers. The observers will never have access to any of your survey responses; they will only view the brief, degraded point-light figures.

Please be reminded that your participation is entirely voluntary. You may choose not to consent to the release of your data. You will not experience any negative consequences if you decide to withdraw from the study, or decide not to release your study data.

\section{Confidentiality:}

All data collected will be kept confidential. The questionnaire responses will be coded in such a way that participants cannot be identified (i.e., each participant will be randomly assigned a number that cannot be linked back to them). Participant gait has been recorded using point-light display technology. The technology is such that only lightreflected markers appear on the recording. Therefore, participants are unrecognizable from observation of the video clips.

The videos will be edited down to a 10-second clip by a trained technician, who has signed a confidentiality agreement. The data will then be stored in a statistical database and kept on a secure computer in Dr. Bennell's research laboratory. The only individuals who will be able to access these files are Dr. Bennell and his research assistants and/or graduate students, who have also signed confidentiality forms ensuring that they will not reveal the content of file information. The data will be kept in locked cabinets or in password protected computer files for five years, as required by the University, before being shredded or deleted. 
Again, please be aware that the current study is part 1 of a multi-part study. The brief walking clips produced in the current study will be shown to a separate sample of undergraduate students who will rate them on various characteristics such as their perceived personality (e.g., confidence), perceived affect (e.g., happiness), and their vulnerability to victimization (sexual and violent). The observers will only see lighted skeletons whom lack any identifying features. The sample of observers will not be told who created the point-light walkers, when they were created, or any background history of the walkers.

\section{Contact for Additional Study Information:}

If you have any questions or desire further information with respect to this study, you may contact:

Brittany Blaskovits at brittanyblaskovits@cmail.carleton.ca, or at 613-520-2600 ext. 1728

or

Dr. Craig Bennell at craig.bennell@carleton.ca, or at 613-520-2600 ext. 1769

This study has received clearance by the Carleton University Ethics Committee for Psychological Research (Research Ethics Approval \#15-057).

If you have any ethical concerns regarding this study, please contact:

Carleton University Ethics Committee for Psychological Research Chair: Dr. Shelley Brown

Phone: 613-520-2600, ext. 1505

Email: shelley.brown@carleton.ca

If you have any other concerns regarding this study, please contact:

Psychology Departmental Chair: Dr. Joanna Pozzulo

Phone: 613-520-2600 ext. 1412

Email: joanna.pozzulo@carleton.ca

\section{Consent:}

Your participation in this study is entirely voluntary and you may refuse to share your data or withdraw from the study.

I have read the above form and hereby consent to allowing my data (including the recorded video and questionnaires) to be used in the current study. I am aware that the data collected in this study will be kept strictly confidential and anonymous. My signature indicates that I understand the above and wish to release my data for the uses indicated above. 
Participant's name

(print):

Participant's signature:

Researcher's name

(print):

Researcher's signature:

Date:

Your data may be used in future studies conducted by colleagues (e.g., to replicate the study with a different observer sample or to replicate the study with animated avatars [i.e., avatars are human-like characters which can be applied over the point-light figures, and then altered to have different clothing, hairstyles, etc]). However, should your data be used in the future, all personal information and/or identifiers will be removed.

All researchers, research assistants, and/or technicians, have signed confidentiality agreements ensuring the security and anonymity of your information. Your recorded walk and survey responses are numerically coded immediately upon their completion and cannot be linked back to you.

I have read the above form and hereby consent to releasing my data (including the recorded video and questionnaires). I am aware that the data collected in this study may be used in future studies, for research publications and/or for teaching purposes. I am aware that all data will be kept strictly confidential and anonymous. My signature indicates that I understand the above and wish to release my data for the uses indicated above.

Participant's name (print):

Participant's signature:

Researcher's name (print):

Researcher's signature:

Date: 
Appendix $\mathbf{J}$

Study 1: Debriefing Form (final)

\section{Debriefing}

\section{Walk This Way: A Kinematic Point-Light Investigation of Victim Vulnerability}

We would like to thank you for participating in this research. Your time and effort are greatly appreciated. This debriefing information is designed to help you understand the nature of this research.

\section{What are we trying to learn in this research?}

Some researchers have suggested that there may be a relationship between gait pattern (e.g., stride length [distance between steps], foot movement [lifted steps, swinging steps], weight shift [transferring weight from one foot to another], etc.) and vulnerability to victimization (e.g., Book, Costello, and Camilleri, 2013). For example, Wheeler, Book, and Costello (2009) found that undergraduate observers were able to detect vulnerability in others from short observations of gait. The present study is interested in further examining the potential link between gait and vulnerability. In addition, the study hopes to reveal whether or not various personality traits (e.g., dominance), and/or affective states (e.g., fear) can translate into walking behaviour, and are noticeable to observers.

\section{Why is this important to psychologists or the general public?}

In determining the nonverbal cues that may make some people more vulnerable to victimization, we can begin to develop intervention strategies that may help to prevent future incidences of victimization (Ritchie, 2014).

\section{How will my data be used?}

The current study is part 1 of a multi-part study. The edited (10-second) walking clips produced in the current study will be shown to a separate sample of undergraduate students who will rate them on various characteristics such as their perceived personality (e.g., confidence), perceived affect (e.g., happiness), and their vulnerability to victimization (sexual and violent). The "walkers" will only appear to the observers as lighted skeletons whom lack any identifying features. The sample of observers will not be told who created the point-light walkers, when they were created, or any background history of the walkers. The observers will never have access to any of your survey responses; they will only view the brief, degraded point-light figures.

Your data may be also be used in future studies conducted by colleagues (e.g., to replicate the study with a different observer sample or to replicate the study with animated avatars [i.e., avatars are human-like characters which can be applied over the 
point-light figures, and then altered to have different clothing, hairstyles, etc]). However, should your data be used in the future, all personal information and/or identifiers will be removed.

Please be reminded that regardless of the findings, you are not at blame for your history, and are in no way responsible for any victimization you may have experienced. The relationship between gait and victimization is unclear; there is absolutely no causal evidence to date which suggests that gait can cause, or lead to, one's victimization. The purpose of the present study is to examine whether or not gait has any influence on vulnerability, as it is possible that there is no relationship. The study is measuring other potential factors (i.e., personality and affect) that may better explain the differential gait styles exhibited by individuals. Given the unclear relationship between gait and victimization, individual feedback (e.g., related to the style of one's walk) cannot be provided.

\section{How can I learn more?}

If you are interested in learning more about perceptions and cues of victim vulnerability please refer to the following sources:

Book, A., Costello, K., \& Camilleri, J. A. (2013). Psychopathy and victim selection: The use of gait as a cue to vulnerability. Journal of Interpersonal Violence, 28(11), 23682383. doi: $10.1177 / 0886260512475315$

Wheeler, S., Book, A., \& Costello, K. (2009). Psychopathic traits and perceptions of victim vulnerability. Criminal Justice and Behavior, 36(6), 635-648. doi: 10.1177/0093854809333958

\section{What if I have questions later?}

If you wish to discuss this research further, please feel free to contact:

Brittany Blaskovits by email: brittanyblaskovits@cmail.carleton.ca

and/or

Dr. Craig Bennell by email: craig.bennell@carleton.ca

If you have any ethical concerns regarding this study, please contact:

Carleton University Ethics Committee for Psychological Research Chair: Dr. Shelley Brown

Phone: 613-520-2600, ext. 1505

Email: shelley.brown@carleton.ca

If you have any other concerns regarding this study, please contact:

Psychology Departmental Chair: Dr. Joanna Pozzulo

Phone: 613-520-2600 ext. 1412

Email: joanna.pozzulo@carleton.ca 


\section{What can I do if I experience discomfort or distress after participating in this study?} Sometimes, filling out psychological measures on our past experiences or emotions can cause us distress or discomfort. Your generosity and willingness to participate in this research is greatly appreciated, however it is not the intent of the researchers to cause you any feelings of distress. If participating in this study made you feel distressed or uncomfortable, and you would like to speak to someone about your thoughts, please feel free to contact:

- Carleton University Health and Counseling Services, located in the Carleton Technology and Training Centre (across from the parking garage), at 613-5206674 (http://www.carleton.ca/health/).

- The Distress Centre of Ottawa and Region at 613-238-3311 (http://www.dcottawa.on.ca)

- The Ottawa Rape Crisis Centre at 613-562-2333 (http://www.orcc.net/)

- The Sexual Assault Support Centre of Ottawa at 613-234-2266 (http://sascottawa.com/default.aspx).

Thank you for taking the time to participate in this study!

Your participation is greatly appreciated! 


\section{Appendix K}

Study 1: Mediation (Baron \& Kenny, 1986)

The decision to conduct ordinal regression, though more difficult to interpret, was necessary given the scaled outcome variable $(0=$ no vulnerable cues present in gait; $5=$ all vulnerable cues present in gait). Since the scaled categories were cumulative in nature, cumulative odds ordinal logistic regression with proportional odds, was employed.

Consistent with Baron and Kenny (1986), Judd and Kenny (1981), and James and Brett (1984), a series of steps were performed to establish whether or not mediation was present. The first step involved in determining whether or not a mediating effect is present requires that one regress the predictor on the outcome (i.e., regress victimization history on gait; path c in Figure 2). If a total effect exists, one can move on to the second and third steps. The second step involves regressing the predictor on the mediator (i.e., regressing victimization history on Assured-Dominance; path a). In the final step, the mediator is regressed on the outcome (i.e., regressing Assured-Dominance on gait; path b), while the predictor is controlled for (i.e., victimization history).

To establish that Assured-Dominance completely mediated the relationship between victimization history and gait, the effect of victimization history on gait (when Assured-Dominance is controlled for; path c') would be zero. Partial mediation is typically established when the direct effect is significant (i.e., path c'), but becomes reduced in size when the mediator is introduced. 
Assured-

Dominance

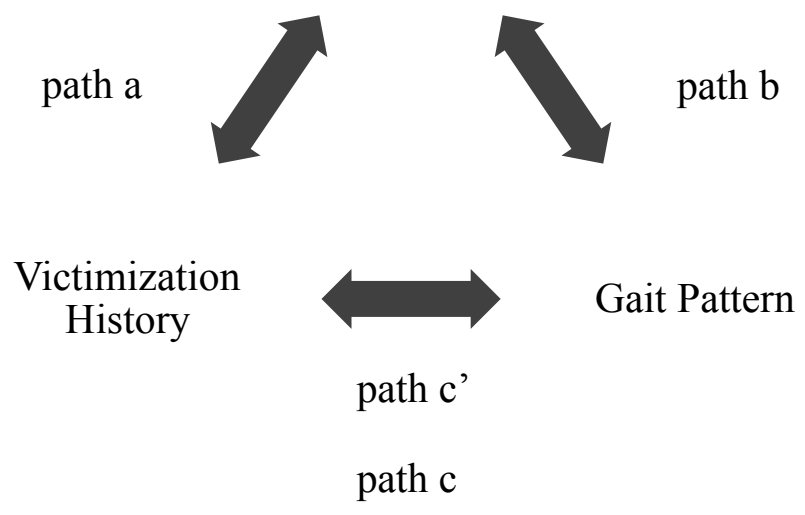

Figure 2. Mediation model examining the relationship between victimization history, gait pattern, and personality

In line with the first step of mediation, victimization history was regressed on the outcome, gait (i.e., estimation and testing of path c). Several assumptions were checked prior to the interpretation of the results, namely the assumption of proportional odds and that of multicollinearity. The Test of Parallel Lines was conducted in order to determine whether or not the assumption of proportional odds was met; it was not significant, $\chi(4)$ $=4.76, p=.313$. Therefore, victimization history had a relatively similar effect at every cumulative split of the outcome variable, gait pattern. Because only one predictor variable was used in the regression model, multicollinearity was not a concern. However, it should be noted that the small sample size resulted in low cell counts (i.e., $<6$ ). Therefore, the chi-square statistic must be interpreted cautiously.

The regression model for path c was significant, $\chi(1)=9.21, p=.002$, suggesting that the predictor variable, victimization history, differed significantly from zero (the null hypothesis). In other words, victimization history had a significant effect on the 
vulnerability level of one's gait pattern. The ordered logit for individuals without a victimization history receiving a vulnerable-coded gait rating was 2.25 (95\% [-3.82, .68]) units less than those with a victimization history, holding all else constant; this result was statistically significant, Wald $\chi^{2}(1)=7.85, p=.005$. In other words, the odds of an individual without a victimization history receiving a vulnerable gait rating was 2.25 times lower than that of someone with a victimization history.

The second step involved regressing victimization history on the mediator, personality (i.e., the Assured-Dominant scale; estimating and testing path a). To ensure that there was a linear relationship present between the predictor and the outcome (the outcome in this case was the mediator, Assured-Dominance), a scatterplot was constructed. Visual inspection of the scatterplot suggested that linearity could be assumed. The scatterplot was also examined for evidence of heteroscedasticity; the error terms along the line of best fit appeared relatively equal and the Q-Q Plot of the standardized residuals were homogeneous. Levene's Test was not significant at the $p<$ .05 level. To test for independence of observation, the Durbin-Watson statistic was obtained. The Durbin-Watson statistic for the model was approximately 1.82; it was thus assumed that autocorrelation was not a concern in the present analyses. Unfortunately, however, the regression model for path a was not significant, $F(1,29)=1.07, p=.310$; victimization history only explained $4 \%$ of the variance in Assured-Dominance ratings, $\left(\mathrm{R}^{2}=.036\right)$.

In step three (estimating and testing path $\mathrm{b}$ and $\mathrm{c}^{\prime}$ ), both victimization history and Assured-Dominance) were entered as the predictors; gait vulnerability was entered as the outcome. The Test of Parallel Lines indicated that the assumption of proportional odds 
was once again met (i.e., it was not significant, $\chi[8]=2.31, p=.970$ ). AssuredDominance was weakly correlated with victimization history $(r=.12)$; thus, multicollinearity was not a major concern. The model was significant, $\chi(2)=13.17, p=$ .001 , and suggested that the predictors (victimization history and Assured-Dominance) had a significant effect on the vulnerability level of one's gait pattern.

The ordered logit for individuals without a victimization history receiving a vulnerable-coded gait rating was 2.00 (95\% [-3.61, -.38]) units less than those with a victimization history, holding all else constant; this result was statistically significant, Wald $\chi^{2}(1)=5.89, p=.015$. In other words, the odds of an individual without a victimization history receiving a vulnerable gait rating was 2 times lower than someone with a victimization history (when Assured-Dominance was controlled for; path c'). On the other hand, the ordered logit for the Assured-Dominant personality variable was .85 (95\% [-.06, 1.77]). Therefore, individuals with higher Assured-Dominant scores, were more likely to receive a vulnerable gait rating, holding all else constant (i.e., when victimization history was controlled for; path b). However, the result was only marginally significant, Wald $\chi^{2}(1)=3.37, p=.067$. Because the mediator did not have a significant effect on gait, nor did it demonstrate a relationship with victimization history, neither complete nor partial mediation could be confirmed. 
Appendix L

Study 2: Victim Vulnerability Rating Questionnaire

Victim Vulnerability Rating Questionnaire

Video \#

On a scale from 1 (not at all vulnerable) to 10 (completely vulnerable), rate the vulnerability of the subject from the clip on the following types of victimization:

$\begin{array}{llllllllll}1 & \underline{2} & \underline{3} & \underline{4} & \underline{5} & \underline{6} & \underline{7} & \underline{8} & \underline{9} & \underline{10}\end{array}$

(not at all vulnerable) $\quad$ (completely vulnerable)

Sexual victimization: Sexual abuse, any non-consensual sexual activity (e.g., sexual assault, rape, etc.), threat of unwanted sexual contact, sexual trafficking, sexual defamation (e.g., sending of nude photos), sexual coercion, etc.

Violent victimization: Physical bullying, in addition to any of the following crimes:

Robbery, mugging, assault, physical abuse, neglect, harassment, battery (e.g., intimate partner violence), kidnapping, gang violence, threats, etc.

Any victimization: Includes all of the above examples of victimization. 
On a scale from 1 (not at all true) to 10 (completely true), rate the subject in the clip on the following statements:

$\begin{array}{llllllllll}1 & \underline{2} & \underline{3} & \underline{4} & \underline{5} & \underline{6} & \underline{7} & \underline{8} & \underline{9} & \underline{10}\end{array}$

(not at all true)

(completely true)

This person is independent:

This person would make an easy target:

This person is strong:

This person needs protection or assistance:

This person would be easily exploited:

This person is capable:

This person is weak:

This person is sad:

This person is unaggressive:

This person is cheerful:

This person is happy:

This person is self-confident:

This person is hostile:

This person is alert:

This person is timid:

This person is assertive:

This person is upset:

This person is afraid:

This person is determined:

Using the same definitions of victimization provided above, state whether the subject is vulnerable to victimization where YES indicates the subject is vulnerable to victimization and NO indicates the subject is not vulnerable to victimization:

Sexual victimization:

$\square$ Yes

$\square$ No

$\neg$ NEXT

If answering any of these questions made you feel distressed or uncomfortable, and you would like to speak to someone about your thoughts, please feel free to contact Carleton University Health and Counseling Services at 613-520-6674 (http://www.carleton.ca/health/) or the Distress Centre of Ottawa and Region at 613-238-3311 (http://www.dcottawa.on.ca). 
Violent victimization:

$\square$ Yes

$\square$ No

Any victimization:

$\square$ Yes

$\square$ No

Using the same definitions of victimization as above, do you think this person has been a victim in the past?

Sexual victimization:

$\square$ Yes

$\square$ No

Violent victimization:

$\square$ Yes

$\square$ No

Any victimization:

$\square$ Yes

$\square$ No 
Appendix M

Study 2: Consent Form

The purpose of an informed consent is to ensure that you understand the purpose of the study and the nature of your involvement. The informed consent must provide sufficient information such that you have the opportunity to determine whether you wish to participate in the study. Please take the time to read this information carefully.

\section{Walk This Way: A Kinematic Point-Light Investigation of Victim Vulnerability}

\section{Research Personnel:}

Brittany Blaskovits

Department of Psychology, Graduate Student, Carleton University

brittanyblaskovits@cmail.carleton.ca

Craig Bennell

Department of Psychology, Faculty, Carleton University

craig.bennell@carleton.ca

\section{Purpose:}

In conducting this study, we hope to gain an understanding of whether observers can accurately detect certain personality traits, affective states, and vulnerability to criminal victimization, from short observations of gait.

\section{Study Procedure:}

You will be asked to complete a short demographic questionnaire before watching a series of 14 brief video-clips. The clips show various point-light display individuals walking. Point-light display is a type of technology that captures body movement while removing extraneous cues (e.g., hair, weight, etc.). The recordings show moving lighted skeletons. Following each clip, you will be asked to provide ratings of the perceived vulnerability of the walkers to various types of victimization (e.g., violent victimization). You will also be asked about the perceived affect and personality of the walkers. At the end of the study you will be debriefed about its purpose. The study will take approximately 30 minutes to complete and you will earn between $\$ 0.80$ and $\$ 1.20$ (depending on where Qualtrics recruited you from) as compensation for your participation. Please note, if you previously participated in a study called "A Kinematic Point-Light Investigation of Memory and Movement," please refrain from signing up to participate in this study. 


\section{Time Required:}

The study will take approximately 30 minutes to complete. You will be directed to the online link upon indicating your consent.

\section{Remuneration:}

You will receive between $\$ 0.80$ and $\$ 1.20$ (depending on where Qualtrics recruited you from) as compensation for your participation.

\section{Right to Withdraw:}

You have the right to withdraw from this study at any point, without penalty. If you choose to withdraw before the end of the study, simply click the "withdraw" button located on each page and it will take you to the debriefing form. You will still receive the full payment for your participation.

\section{Potential Risks:}

The following risks are associated with participating in the study: You will be asked to provide ratings of the perceived vulnerability of the walkers in the video clips to various types of victimization (e.g., violent victimization). If you anticipate that providing such ratings may cause you discomfort, you may choose not to participate. In the event that you do choose to participate and feel uncomfortable at any point during the study, please be reminded that your participation is entirely voluntary and you are not required to answer any question in the surveys. You may withdraw at any time if you no longer wish to participate. You will not experience any negative consequences if you decide to withdraw from the study, or decide not to answer any specific questions.

\section{Confidentiality:}

All responses will be strictly confidential. The data may be used for research publications, conference presentations and/or teaching material. However, all answers will be coded in such a way that participants cannot be identified. IP addresses will not be collected during this study. Upon reaching the debriefing form, Qualtrics will automatically credit your account between $\$ 0.80$ and $\$ 1.20$ (depending on where Qualtrics recruited you from). Your account information will not be linked to your survey responses.

The data is collected through the software Qualtrics, which uses servers with multiple layers of security to protect the privacy of the data (e.g., encrypted websites and password protected storage). Please note that Qualtrics is hosted by a server located in the USA. The United States Patriot Act permits U.S. law enforcement officials, for the purpose of an anti-terrorism investigation, to seek a court order that allows access to the personal records of any person without that person's knowledge. In view of this we cannot absolutely guarantee the full confidentiality and anonymity of your data. With your consent to participate in this study you acknowledge this. 
The data will be stored in a statistical database and kept on a secure computer in Dr. Bennell's research laboratory. The only individuals who will be able to access these files are Dr. Bennell, his research assistants and/or his graduate students, who have signed confidentiality forms ensuring that they will not reveal the content of file information. The data will be kept in locked cabinets or in password protected computer files for five years, as required by the University, before being shredded or deleted.

\section{Contact for Additional Study Information:}

If you have any questions or desire further information with respect to this study, you may contact:

Brittany Blaskovits at brittanyblaskovits@cmail.carleton.ca, or at 613-520-2600 ext. 1728

or

Dr. Craig Bennell at craig.bennell@carleton.ca, or at 613-520-2600 ext. 1769

This study has received clearance by the Carleton University Ethics Committee for Psychological Research (Research Ethics Approval \#15-057).

If you have any ethical concerns regarding this study, please contact:

Carleton University Ethics Committee for Psychological Research Chair: Dr. Shelley Brown

Phone: 613-520-2600, ext. 1505

Email: shelley.brown@carleton.ca

If you have any other concerns regarding this study, please contact:

Psychology Departmental Chair: Dr. Joanna Pozzulo

Phone: 613-520-2600 ext. 1412

Email: joanna.pozzulo@carleton.ca

\section{Consent:}

Your participation in this study is entirely voluntary and you may refuse to participate or withdraw from the study at any time.

I have read the above form and hereby consent to continue participating in this study. The data in this study may be used for research publications and/or for teaching purposes. I am aware that the data collected in this study will be kept strictly confidential and anonymous. By checking the box I consent to participate in this study.

By checking this box I indicate that I have read the above informed consent and that I am fully aware of any possible risks or consequences of participating in this study. 


\section{Appendix N}

Study 2: Instructions

\section{Instructions}

You will be asked to complete a short demographic questionnaire before watching a series of 14 brief, unordered video-clips. Each clip is approximately 10 seconds long and shows a different point-light figure walking. Following each clip, you will be asked to provide ratings of the perceived vulnerability of the walker to various types of victimization (e.g., violent victimization). You will also be asked about the perceived affect and personality of the walkers.

You may take as much time as necessary to complete the Victim Vulnerability Rating Questionnaire following each clip. When you are ready to observe the next video, simply select the "Next" button at the bottom of the web page. If, at any time, you feel uncomfortable, you may choose to select either the "Skip" or "Withdraw" options that are also present at the bottom of each web page.

At the end of the study you will be debriefed about its purpose. The study will take approximately 30 minutes to complete and you will earn between $\$ 0.80$ and $\$ 1.20$ as compensation for your participation. Your generosity and willingness to participate in this research is greatly appreciated 
Appendix O

Study 2: Online Debriefing Form

\section{Debriefing}

\section{Walk This Way: A Kinematic Point-Light Investigation of Victim Vulnerability}

We would like to thank you for participating in this research. Your time and effort are greatly appreciated. This debriefing information is designed to help you understand the nature of this research.

\section{What are we trying to learn in this research?}

Recent research suggests that individuals are able to detect vulnerability in others from short observations of gait (e.g., Book, Costello, \& Camilleri, 2013). For example, Wheeler, Book, and Costello (2009) recorded brief videos of students walking down a hallway. They found that observers rated certain gait patterns as more vulnerable than others. Researchers have thus suggested that there may be a relationship between gait pattern (e.g., stride length [distance between steps], foot movement [lifted steps, swinging steps], weight shift [transferring weight from one foot to another], etc.) and vulnerability to victimization.

Currently however, it is unclear if observers are making judgements based solely on gait movement cues, or if there are other variables confounding victim choice (e.g., age, attractiveness, etc.). Point-light display technology captures biological human body movement while removing extraneous cues (e.g., hair, weight, sex, etc.), thereby providing a clearer study of gait analysis and observer detection ability.

The present research aims to confirm that individuals agree on the target for future victimization, and that ratings are accurate to the extent that those chosen as future victims correlate with self-reported victimization experiences of the walkers. A secondary aim of the study is to examine whether or not various personality traits (e.g., dominance) and/or affective states (e.g., fear) can translate into walking behaviour, and are noticeable to observers. Some research has suggested that vulnerable gait patterns may be the product of a submissive personality (e.g., Myers, Templer, \& Brown, 1984). However, another body of literature has indicated both the translation of affective states into gait, as well as the accuracy of zero-acquaintance observers in detecting affect from gait (e.g., Ikeda \& Watanabe, 2009; Schneider et al., 2014). Therefore, the present study will be the first to examine the detection accuracy of personality traits versus affect, from gait cues.

\section{Why is this important to psychologists or the general public?}

If observers select previous victims as future victims using only the point-light stimuli, there will be additional evidence suggesting that individuals can infer vulnerability from 
gait cues. We can then begin to develop intervention strategies that may help to prevent future incidences of victimization (Ritchie, 2014).

\section{How can I learn more?}

If you are interested in learning more about perceptions and cues of victim vulnerability please refer to the following sources:

Book, A., Costello, K., \& Camilleri, J. A. (2013). Psychopathy and victim selection: The use of gait as a cue to vulnerability. Journal of Interpersonal Violence, 28(11), 23682383. doi: $10.1177 / 0886260512475315$

Wheeler, S., Book, A., \& Costello, K. (2009). Psychopathic traits and perceptions of victim vulnerability. Criminal Justice and Behavior, 36(6), 635-648. doi: 10.1177/0093854809333958

\section{What if I have questions later?}

If you wish to discuss this research further feel free to contact:

Brittany Blaskovits by email: brittanyblaskovits@cmail.carleton.ca and/or

Dr. Craig Bennell by email craig.bennell@carleton.ca

If you have any ethical concerns regarding this study, please contact:

Carleton University Ethics Committee for Psychological Research Chair: Dr. Shelley Brown

Phone: 613-520-2600, ext. 1505

Email: shelley.brown@carleton.ca

If you have any other concerns regarding this study, please contact:

Psychology Departmental Chair: Dr. Joanna Pozzulo

Phone: 613-520-2600 ext. 1412

Email: joanna.pozzulo@carleton.ca

\section{What can I do if I experience discomfort or distress after participating in this study?}

Sometimes, filling out psychological measures can cause us distress or discomfort. Your generosity and willingness to participate in this research is greatly appreciated, however it is not the intent of the researchers to cause you any feelings of distress. If participating in this study made you feel distressed or uncomfortable, and you would like to speak to someone about your thoughts, please feel free to contact:

- Carleton University Health and Counseling Services, located in the Carleton Technology and Training Centre (across from the parking garage), at 613-5206674 (http://www.carleton.ca/health/).

- The Distress Centre of Ottawa and Region at 613-238-3311 (http://www.dcottawa.on.ca)

- The Ottawa Rape Crisis Centre at 613-562-2333 (http://www.orcc.net/) 
- The Sexual Assault Support Centre of Ottawa at 613-234-2266 (http://sascottawa.com/default.aspx).

Thank you for taking the time to participate in this study! 